Prepared in cooperation with Broward County, Florida

\title{
Lithofacies and Sequence Stratigraphic Description of the Upper Part of the Avon Park Formation and the Arcadia Formation in U.S. Geological Survey G-2984 Test Corehole, Broward County, Florida
}

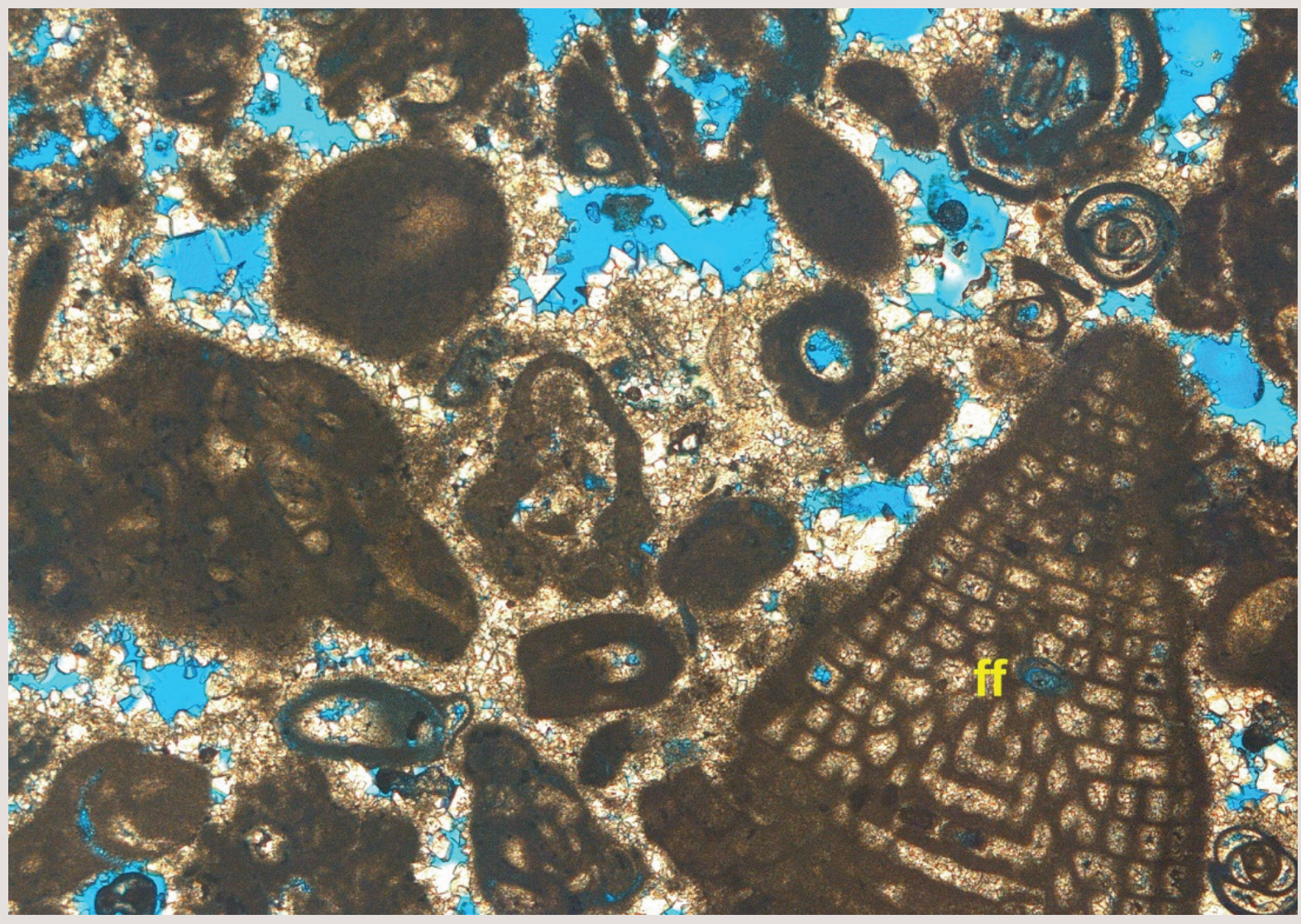

Open-File Report 2017-1074 
Cover. Photomicrograph from thin section G2984-1074.00 that shows smaller and larger benthic foraminifer grainstone. This interparticle-dominated pore system produces diffuse flow in part of the upper Floridan aquifer composed of the Avon Park Formation. The large foraminiferal specimen in the lower right is Fallotella floridana (ff). Driller's depth of thin section is 1,074.00 feet below land surface. 


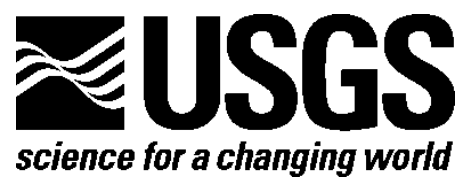

Prepared in cooperation with Broward County, Florida

\section{Lithofacies and Sequence Stratigraphic Description of the Upper Part of the Avon Park Formation and the Arcadia Formation in U.S. Geological Survey G-2984 Test Corehole, Broward County, Florida}

By Kevin J. Cunningham and Edward Robinson

Open File-Report 2017-1074

U.S. Department of the Interior U.S. Geological Survey 


\section{U.S. Department of the Interior \\ RYAN K. ZINKE, Secretary}

\section{U.S. Geological Survey \\ William H. Werkheiser, Acting Director}

U.S. Geological Survey, Reston, Virginia: 2017

For more information on the USGS-the Federal source for science about the Earth, its natural and living resources, natural hazards, and the environment-visit http://www.usgs.gov/ or call 1-888-ASK-USGS (1-888-275-8747).

For an overview of USGS information products, including maps, imagery, and publications, visit https://store.usgs.gov/.

Any use of trade, firm, or product names is for descriptive purposes only and does not imply endorsement by the U.S. Government.

Although this information product, for the most part, is in the public domain, it also may contain copyrighted materials as noted in the text. Permission to reproduce copyrighted items must be secured from the copyright owner.

Suggested citation:

Cunningham, K.J., and Robinson, Edward, 2017, Lithofacies and sequence stratigraphic description of the upper part of the Avon Park Formation and the Arcadia Formation in U.S. Geological Survey G-2984 test corehole, Broward County, Florida: U.S. Geological Survey Open File-Report 2017-1074, 139 p., https://doi.org/10.3133/ofr20171074.

ISSN 2331-1258 (online) 


\section{Acknowledgments}

The authors thank Michael Zygnerski, Barbara Powell, and Jennifer Jurado of the Broward County Environmental Protection and Growth Management Department-Environmental Planning and Community Resilience Division for providing guidance during the course of the project. Jonathan Arthur and David Paul of the Florida Geological Survey granted permission to examine the core from the G-2984 test corehole. Jacqueline Powell, U.S. Geological Survey (USGS) volunteer, assisted with core-sample processing and provided early guidance in identification of benthic foraminifera. Richard Westcott of Cherokee Nation Technology Solutions bisected core samples with a rock saw to create slabbed core samples and cut core samples for thin section preparation. Richard Westcott and Shakira Khan of Cherokee Nation Technology Solutions and Kim Swidarski and Joann Dixon of the USGS provided invaluable assistance with construction of figures. G. Lynn Wingard of the USGS Eastern Geology and Paleoclimate Science Center provided identification of an oyster of the Gryphaeidae family. 


\section{Contents}

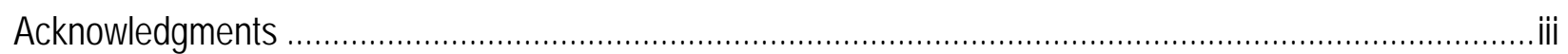

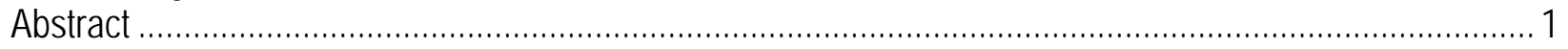

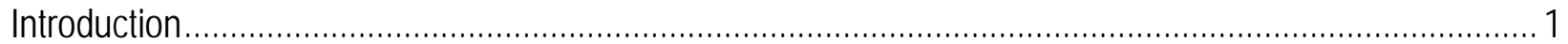

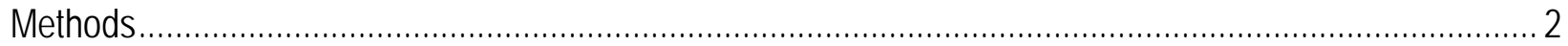

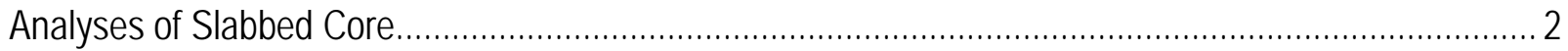

Foraminiferal Paleontologic Analysis ........................................................................................ 5

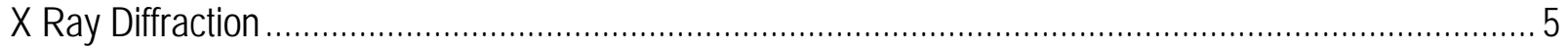

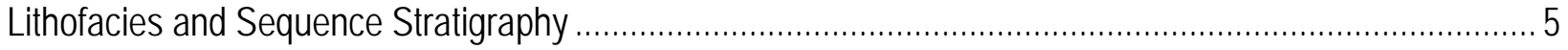

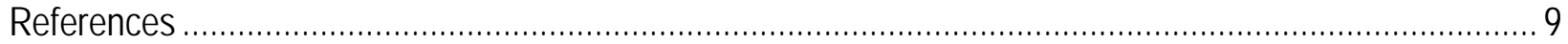

Lithofacies Description and Sequence Stratigraphy of Continuously Drilled Samples from the

Avon Park Formation at U.S. Geological Survey G-2984 Test Corehole............................................. 11

Lithofacies Description and Sequence Stratigraphy of Continuously Drilled Samples from the

Arcadia Formation at U.S. Geological Survey G-2984 Test Corehole ................................................. 94

\section{Figures}

Figure 1. Map showing location of U.S. Geological Survey G-2984 test corehole in southeastern

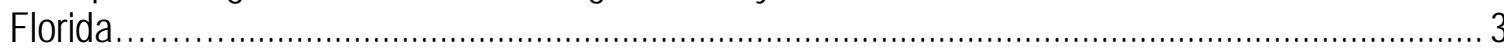

Figure 2. Stratigraphic chart showing correlation of chronostratigraphy, geologic units, lithology, sequence stratigraphy, major cycles, depositional cycles, eustatic sea-level curve, seismic horizons, and hydrogeologic units of U.S. Geological Survey G-2984 test corehole

Figure 3. Diagrams showing two of three ideal high-frequency cycles defined for depositional sequence DS AP2 of the Avon Park Formation by analyzing slabbed core from U.S. Geological Survey G-2984 test corehole

Figure 4. Diagrams showing two of four ideal high-frequency cycles defined for depositional sequence DS AP2 and DS AP3 of the Avon Park Formation by analyzing slabbed core from U.S. Geological Survey G-2984 test corehole

\section{Tables}

Table 1. X ray diffraction results for rock core samples from the Arcadia Formation in U.S. Geological Survey G-2984 test corehole. 


\section{Conversion Factors}

U.S. customary units to International System of Units

\begin{tabular}{|c|c|c|}
\hline Multiply & By & To obtain \\
\hline \multicolumn{3}{|c|}{ Length } \\
\hline inch (in.) & 2.54 & centimeter $(\mathrm{cm})$ \\
\hline inch (in.) & 25.4 & millimeter (mm) \\
\hline foot $(\mathrm{ft})$ & 0.3048 & meter (m) \\
\hline \multicolumn{3}{|c|}{ Mass } \\
\hline ounce, avoirdupois (oz) & 28.35 & gram (g) \\
\hline
\end{tabular}

\section{Abbreviations}

$\begin{array}{ll}\text { bls } & \text { below land surface } \\ \text { Ca } & \text { calcium } \\ \text { DS } & \text { depositional sequence } \\ \text { Fe } & \text { iron } \\ \text { obi } & \text { optical image logger } \\ \text { USGS } & \text { U.S. Geological Survey } \\ \text { XRD } & \text { x ray diffraction }\end{array}$




\title{
Lithofacies and Sequence Stratigraphic Description of the Upper Part of the Avon Park Formation and the Arcadia Formation in U.S. Geological Survey G-2984 Test Corehole, Broward County, Florida
}

\author{
By Kevin J. Cunningham and Edward Robinson
}

\begin{abstract}
Rock core and sediment from U.S. Geological Survey test corehole G-2984 completed in 2011 in Broward County, Florida, provide an opportunity to improve the understanding of the lithostratigraphic, sequence stratigraphic, and hydrogeologic framework of the intermediate confining unit and Floridan aquifer system in southeastern Florida. A multidisciplinary approach including characterization of sequence stratigraphy, lithofacies, ichnology, foraminiferal paleontology, depositional environments, porosity, and permeability was used to describe the geologic samples from this test corehole. This information has produced a detailed characterization of the lithofacies and sequence stratigraphy of the upper part of the middle Eocene Avon Park Formation and Oligocene to middle Miocene Arcadia Formation. This enhancement of the knowledge of the sequence stratigraphic framework is especially important, because subaerial karst unconformities at the upper boundary of depositional cycles at various hierarchical scales are commonly associated with secondary porosity and enhanced permeability in the Floridan aquifer system.
\end{abstract}

\section{Introduction}

The U.S. Geological Survey (USGS) G-2984 test corehole (Florida Geological Survey well ascension number W-17986) was completed in November 2011 by the Florida Geological Survey in Broward County, Florida (fig. 1). The main purpose for drilling the test corehole was to provide additional information to update the lithostratigraphic, sequence stratigraphic, and hydrogeologic framework of the upper part of the Upper Floridan aquifer for a cooperative investigation by the USGS and Broward County completed by Reese and Cunningham (2014). The test corehole was continuously cored from land surface to a total depth of 1,308 feet below land surface (ft bls). The corehole penetrated middle Eocene, Oligocene, Neogene, and Quaternary rocks and sediments. These strata include the surficial aquifer, intermediate confining unit, and the upper part of the Upper Floridan aquifer. The purpose of this report is to describe the sequence stratigraphy, lithofacies, ichnology, foraminiferal paleontology, depositional environments, porosity, and permeability of the upper part of the Avon Park Formation and Arcadia Formation by using data obtained from the core (fig. 2). These data can be used as multiple lines of evidence for advancing the understanding of the Eocene to Miocene 
sequence stratigraphic, lithostratigraphic, and hydrogeologic framework of the southeastern Florida Platform. Notably, the sequence stratigraphic framework is important because subaerial karst unconformities at the upper boundary of depositional cycles at various hierarchical scales (Kerans and Tinker, 1997, fig. 1.11) are commonly associated with secondary porosity and enhanced permeability in the Floridan aquifer system (Cander, 1995; Reese and Cunningham, 2014) and in many oil and gas fields around the world (Budd and others, 1995).

\section{Methods}

A multidisciplinary approach was used to describe the lithofacies and sequence stratigraphy of the upper part of the Avon Park Formation and Arcadia Formation. This approach used data from analyses of slabbed core, including sequence stratigraphy, paleontology (emphasis on foraminifera), x ray diffraction (XRD), ichnology, and porosity and permeability characterization. At the time of this publication, all core samples and thin sections used in this study are temporarily stored at the U.S. Geological Survey Florida Water Science Center, Fort Lauderdale, Fla. Permanent storage of the core samples and thin sections will be at the Florida Geologic Survey Geologic Core Repository, Tallahassee, Fla.

\section{Analyses of Slabbed Core}

Numerous slabbed core samples (approximately 2-inch [in.] diameter) from an upper part of the Avon Park Formation and the entire thickness of the Arcadia Formation were analyzed. All of the core samples were first visually analyzed by using a 10x-magnification hand lens and a binocular microscope, and then compared to optical and acoustic borehole wall images acquired by a wireline logging tool. The total magnification of the hand lens is multiplied by 10 , as indicated by “10x". Standard transmitted-light petrography was used to examine 179 thin sections. The slabbed cores and thin sections were useful for determining lithofacies, vertical trends in lithofacies, sedimentary structures, ichnology, and sequence stratigraphic (cycle) boundaries. Lithofacies were identified by grain types, fabric, depositional texture, and diagenetic features by using a combination of classification schemes and terminology from Dunham (1962), Embry and Klovan (1971), and Lucia (1999). Comparison of slabbed core to a Munsell rock color chart (Geological Society of America, 1991) was used to record rock color. A semiquantitative field classification of ichnofabric (Droser and Bottjer, 1986, 1989) was used to record variations in the extent of bioturbation. Classifications of porosity were assessed by using schemes developed by Choquette and Pray (1970) and Lucia (1995, 1999). A total of 276 airpermeability measurements (Cunningham and others, 2006) and calculated lattice Boltzman permeability values of both Pleistocene and Cretaceous carbonate rocks (Cunningham and others, 2009, 2012; Cunningham and Sukop, 2011, 2012; Sukop and others, 2013; Sukop and Cunningham, 2014) were used as reference material for the purpose of visual comparison to the Avon Park and Arcadia Formation core samples from the G-2984 test corehole in order to visually estimate the permeability of these samples. The Pleistocene and Cretaceous carbonate rock samples have similar lithofacies and pore types as the core samples described herein from the G-2984 test corehole. 


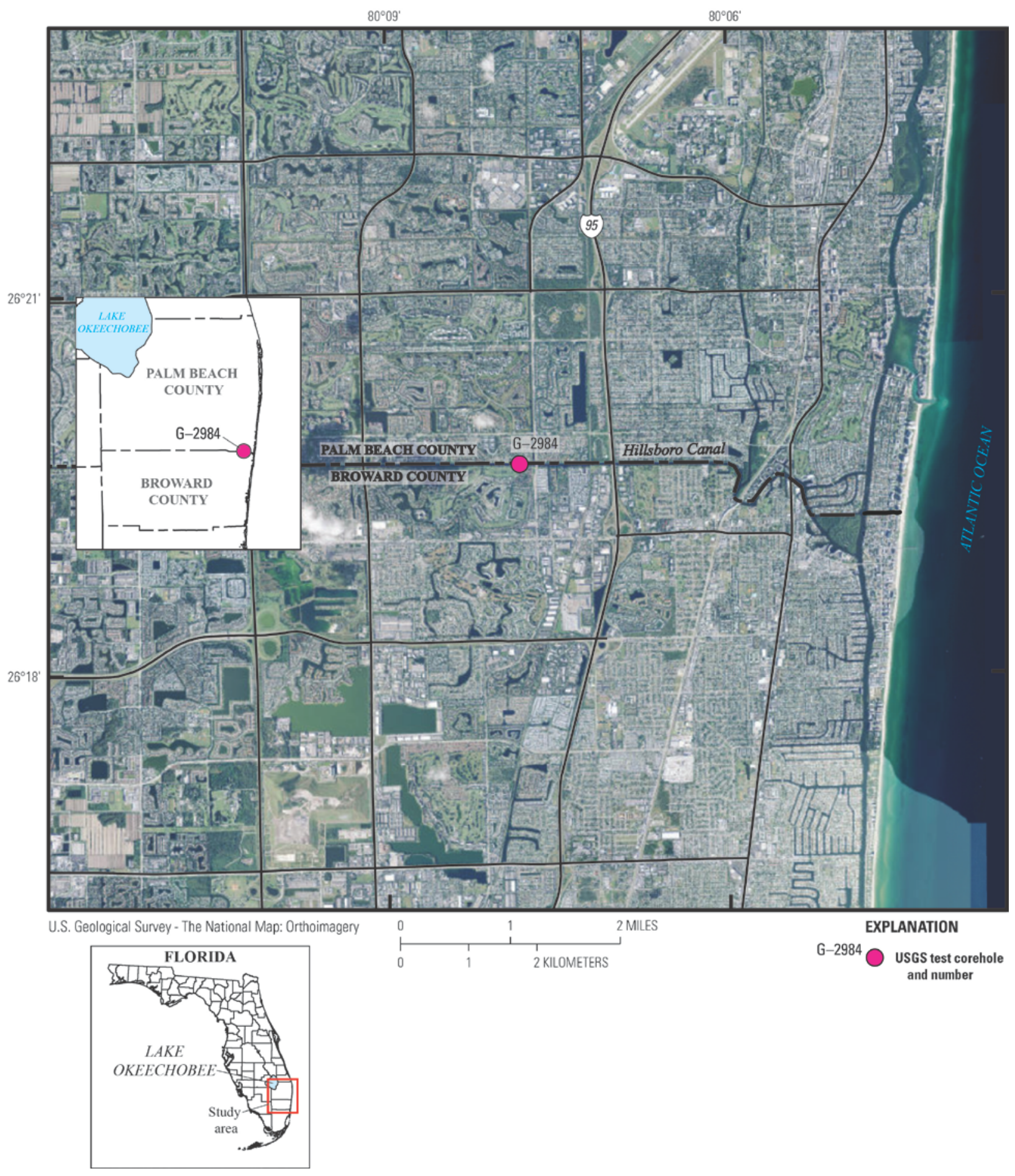

Figure 1. Map showing location of U.S. Geological Survey G-2984 test corehole in southeastern Florida. 


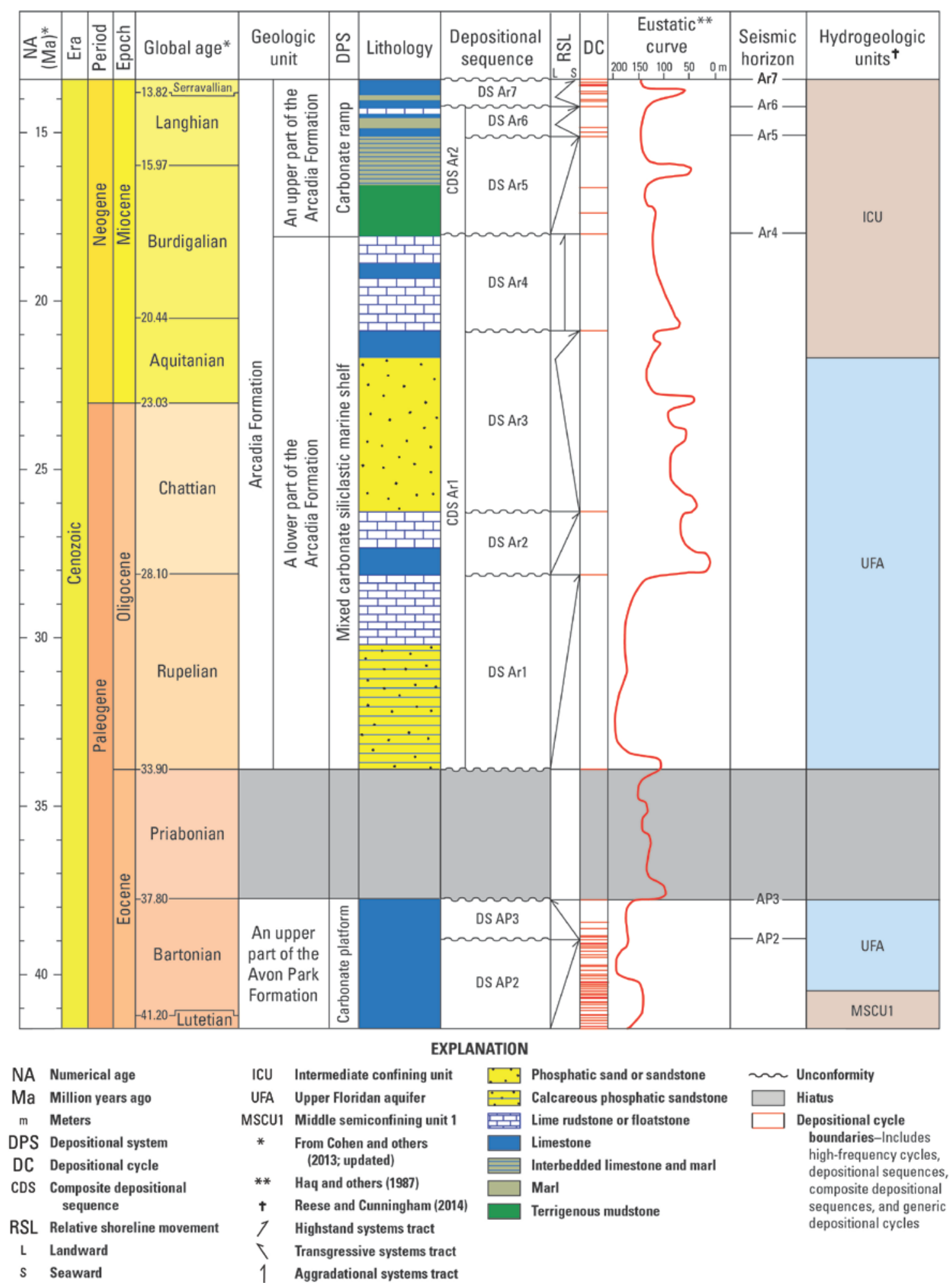

Figure 2. Stratigraphic chart showing correlation of chronostratigraphy, geologic units, lithology, sequence stratigraphy, major cycles, depositional cycles, eustatic sea-level curve, seismic horizons, and hydrogeologic units of U.S. Geological Survey G-2984 test corehole. Note that the hiatus represents an interruption in the geologic record, such as nondeposition, erosion, or both. 


\section{Foraminiferal Paleontologic Analysis}

Taxonomy of benthic and planktic foraminifera from selected lithofacies was determined to assist in interpretation of depositional environments and biostratigraphy. Foraminifera were examined in 189 thin sections prepared from core samples acquired from the G-2984 test corehole (fig. 1). Thin sections were examined by transmitted light by using a Zeiss stereoscopic microscope and by counting selected taxa at magnifications ranging from $1.6 \mathrm{x}$ to $6.3 \mathrm{x}$. Thin sections were then examined by using an AmScope 2000X LED Model SME-F8BH trinocular compound microscope for more detailed analysis and identification to the highest practicable taxonomic level. Typical examples of identified taxa were imaged by using an AmScope MU Series 10-megapixel digital camera.

\section{Ray Diffraction}

Twenty-four rock samples were analyzed by XRD to identify the clay and carbonate content of the rock samples acquired from the Arcadia Formation in the G-2984 test corehole (fig. 1, table 1). Use of XRD provided a quantitative guide for differentiating marl and limestone, and assisted in characterizing depositional environments of the carbonate ramps that compose the Arcadia Formation. Marl is a lithology consisting of 65-35 percent carbonate and 35-65 percent clay (Pettijohn, 1957; Flügel, 2004). Samples weighed between 10.2 and 30.0 grams and were used for determination of weight percentage whole-rock and clay-fraction mineralogy by XRD analytical procedures at Core Laboratories LP in Houston, Texas. Each sample was cleaned of any observable drilling contaminants and then disaggregated with a mortar and pestle. Approximately 5 grams of each sample was transferred to isopropyl alcohol and ground in a McCrone micronizing mill. A split of the resultant powders was dried, disaggregated, and backloaded into aluminum sample holders to produce whole-rock mounts. A separate split of each sample powder was dispersed in a dilute sodium-phosphate solution by using a sonic probe, and the suspensions were then centrifugally size-fractionated to isolate clay-size (less than 4 microns) materials. The separate clay-fraction suspensions were then vacuum deposited on silver membrane filters to produce clay-oriented mineral aggregates and attached to stainless steel slugs.

\section{Lithofacies and Sequence Stratigraphy}

The fundamental lithostratigraphic component identified in this report is the lithofacies. A lithofacies is a lateral mappable subdivision of a designated stratigraphic unit, distinguished from adjacent subdivisions on the basis of lithology, including all mineralogic and petrographic characteristics and those paleontologic characteristics that influence the appearance, composition, or texture of the rock (Neuendorf and others, 2005). Unique, vertically defined lithofacies units were identified and described in a concise abbreviated style for the Avon Park Formation and Arcadia Formation core samples from the G-2984 test corehole, and are presented in the final two sections herein. The lithofacies units stack into vertical lithofacies successions that contain a record of shallowing upward or deepening upward environments, or an amalgamation of a persistent, aggradational environment as accommodation is filled within a cycle-scale relative change in sea level (Kerans and Tinker, 1997, fig. 1.11).

In the two succeeding sections of this report that contain the core descriptions of the Avon Park and Arcadia Formations, information is presented in a two-column display that 
Table 1. X ray diffraction results for rock core samples from the Arcadia Formation in U.S. Geological Survey G-2984 test corehole.

[Fe, iron; Ca, calcium]

\begin{tabular}{|c|c|c|c|c|c|c|c|c|c|c|c|c|}
\hline \multirow{2}{*}{$\begin{array}{l}\text { Driller's } \\
\text { depth } \\
\text { (feet } \\
\text { below } \\
\text { land } \\
\text { surface) }\end{array}$} & \multirow[b]{2}{*}{$\begin{array}{l}\text { Seismic } \\
\text { sequence }\end{array}$} & \multicolumn{7}{|c|}{$\begin{array}{l}\text { Whole-rock mineralogy } \\
\text { (weight percentage) }\end{array}$} & \multicolumn{4}{|c|}{$\begin{array}{l}\text { Clay mineralogy } \\
\text { (weight percentage) }\end{array}$} \\
\hline & & Quartz & $\begin{array}{c}\text { K- } \\
\text { feldspar }\end{array}$ & $\begin{array}{l}\text { Plagio- } \\
\text { clase }\end{array}$ & Calcite & $\begin{array}{c}\text { Dolomite } \\
\text { and } \\
\text { (Fe,Ca)- } \\
\text { dolomite }\end{array}$ & $\begin{array}{l}\text { Fluora- } \\
\text { patite }\end{array}$ & $\begin{array}{l}\text { Total } \\
\text { clay } \\
\text { miner- } \\
\text { als }\end{array}$ & $\begin{array}{l}\text { Illitel } \\
\text { smec- } \\
\text { tite* }^{*}\end{array}$ & $\begin{array}{l}\text { Sepi- } \\
\text { olite }\end{array}$ & $\begin{array}{l}\text { Palygor- } \\
\text { skite }\end{array}$ & $\begin{array}{l}\text { Kaoli- } \\
\text { nite }\end{array}$ \\
\hline 606.76 & Ar7 & 4.8 & 0.0 & 0.0 & 64.6 & 16.9 & 0.0 & 13.7 & 3.8 & 0.0 & 9.9 & 0.0 \\
\hline 611.90 & Ar7 & 9.6 & 1.0 & 1.7 & 57.2 & 16.7 & 0.0 & 13.8 & 4.5 & 0.0 & 9.3 & 0.0 \\
\hline 619.50 & Ar7 & 9.1 & 0.0 & 0.0 & 15.0 & 29.7 & 0.0 & 46.2 & 20.6 & 0.0 & 25.2 & 0.4 \\
\hline 624.07 & Ar7 & 8.0 & 1.3 & 2.3 & 59.7 & 15.1 & 0.0 & 13.6 & 4.4 & 0.0 & 9.2 & 0.0 \\
\hline 644.87 & Ar7 & 6.4 & 0.7 & 1.2 & 81.6 & 2.3 & 0.0 & 7.8 & 2.7 & 0.0 & 5.1 & 0.0 \\
\hline 654.15 & Ar7 & 5.4 & 0.0 & 0.0 & 68.6 & 3.1 & 0.0 & 22.9 & 10.7 & 0.0 & 12.2 & 0.0 \\
\hline 662.97 & Ar7 & 14.1 & 0.0 & 3.7 & 26.7 & 8.2 & 0.0 & 47.3 & 15.6 & 0.0 & 30.6 & 1.1 \\
\hline 678.70 & Ar7 & 9.9 & 0.0 & 2.0 & 43.9 & 7.1 & 0.0 & 37.1 & 12.4 & 0.0 & 23.8 & 0.9 \\
\hline 689.90 & Ar7 & 4.9 & 0.0 & 0.0 & 64.1 & 3.3 & 1.1 & 26.5 & 12.4 & 0.0 & 14.1 & 0.0 \\
\hline 691.00 & Ar7 & 4.7 & 0.0 & 0.0 & 70.3 & 2.6 & 0.0 & 22.4 & 7.2 & 0.0 & 15.2 & 0.0 \\
\hline 696.00 & Ar7 & 2.0 & 0.0 & 0.0 & 79.8 & 1.1 & 2.8 & 14.3 & 4.3 & 0.0 & 10.0 & 0.0 \\
\hline 698.64 & Ar7 & 3.8 & 0.0 & 1.1 & 48.2 & 10.6 & 0.0 & 36.3 & 12.3 & 0.0 & 24.0 & 0.0 \\
\hline 708.00 & Ar7 & 3.3 & 0.0 & 0.0 & 76.3 & 1.6 & 0.0 & 18.8 & 7.2 & 0.0 & 11.6 & 0.0 \\
\hline 718.00 & Ar6 & 2.2 & 0.0 & 0.0 & 87.4 & 0.7 & 0.0 & 9.7 & 3.3 & 0.0 & 6.4 & 0.0 \\
\hline 767.80 & Ar6 & 3.8 & 0.0 & 0.0 & 42.3 & 10.2 & 0.0 & 43.8 & 8.6 & 14.0 & 21.2 & 0.0 \\
\hline 805.35 & Ar6 & 1.7 & 0.0 & 0.7 & 23.8 & 19.0 & 0.0 & 54.9 & 8.1 & 17.6 & 29.2 & 0.0 \\
\hline 812.10 & Ar6 & 2.5 & 0.0 & 0.0 & 21.3 & 16.6 & 0.0 & 59.6 & 6.0 & 23.1 & 30.5 & 0.0 \\
\hline 828.70 & Ar6 & 2.0 & 0.0 & 0.0 & 28.3 & 26.7 & 0.0 & 43.0 & 7.8 & 20.3 & 14.9 & 0.0 \\
\hline 841.60 & Ar6 & 1.7 & 0.0 & 0.0 & 37.7 & 19.2 & 0.0 & 41.5 & 8.4 & 23.0 & 10.1 & 0.0 \\
\hline 850.85 & Ar6 & 2.1 & 0.0 & 1.0 & 52.1 & 19.8 & 0.0 & 25.0 & 0.0 & 13.9 & 11.1 & 0.0 \\
\hline 871.40 & Ar6 & 2.2 & 0.0 & 0.0 & 44.4 & 14.7 & 0.0 & 38.6 & 6.3 & 22.3 & 10.0 & 0.0 \\
\hline 898.00 & Ar5 & 1.6 & 0.0 & 0.0 & 26.1 & 14.7 & 0.0 & 57.6 & 8.2 & 23.7 & 25.7 & 0.0 \\
\hline 914.65 & Ar5 & 1.8 & 0.0 & 0.0 & 0.8 & 9.6 & 0.0 & 87.8 & 10.9 & 16.4 & 60.5 & 0.0 \\
\hline 923.95 & Ar5 & 2.4 & 0.0 & 0.0 & 2.7 & 3.8 & 0.0 & 91.0 & 6.9 & 13.0 & 71.1 & 0.0 \\
\hline
\end{tabular}

*Mixed-layer illite/smectite contains 70-80 percent smectite layers.

provides depths in the left column and lithofacies descriptions in the right column. The tops and bottoms of lithofacies units that compose vertical lithofacies successions are separated by thin horizontal black lines, whereas bold horizontal black lines are used to mark the boundaries where a vertical lithofacies succession boundary coexists with a sequence stratigraphic (cyclostratigraphic) boundary.

The hierarchy of sequence stratigraphy applied herein is based on the terminology scheme of Kerans and Tinker (1997, fig. 1.11) and presented from the highest cycle hierarchical order to the lowest. The hierarchy includes high-frequency cycle, depositional sequence, and composite depositional sequence (fig. 2). Where the relative position of a specific cycle within this cycle hierarchy has a low level of confidence, the term "depositional cycle" is used. The hierarchical ordering of the cycle types indicates relative scale and position in the cycle hierarchy, but no particular time duration for generic depositional cycle types is inferred. Four different ideal high-frequency cycle types are defined for the Avon Park Formation. These ideal cycle types are herein named type I, type II, type III, and type IV and can be described as follows: (1) type I cycles are microbial laminite-capped grain-rich peritidal cycles; (2) type II cycles are rhizolith- and mud-capped micrite-rich peritidal cycles; (3) type III cycles are aggradational grain-rich subtidal cycles; and (4) type IV cycles are Glossifungites-capped subtidal cycles (figs. 3 and 4 ). 
Microbial laminite-capped grain-rich peritidal cycle (type I cycle)

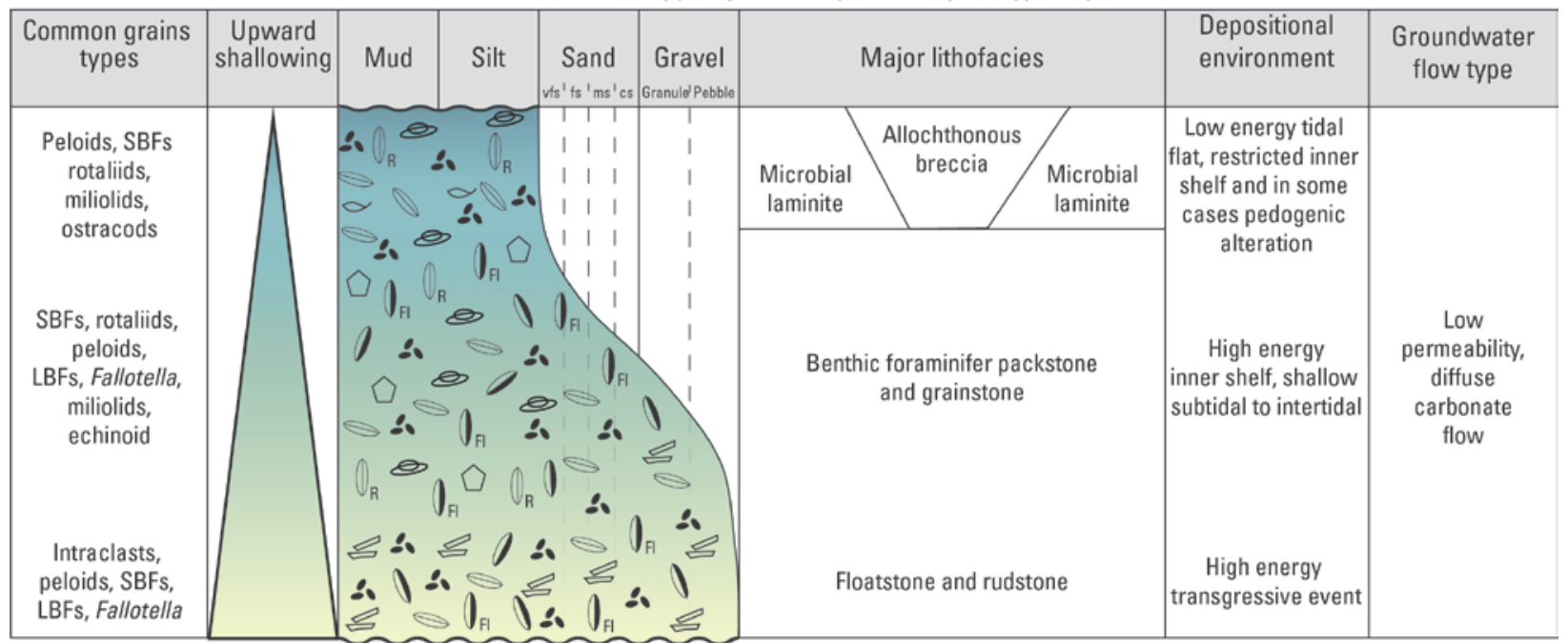

B

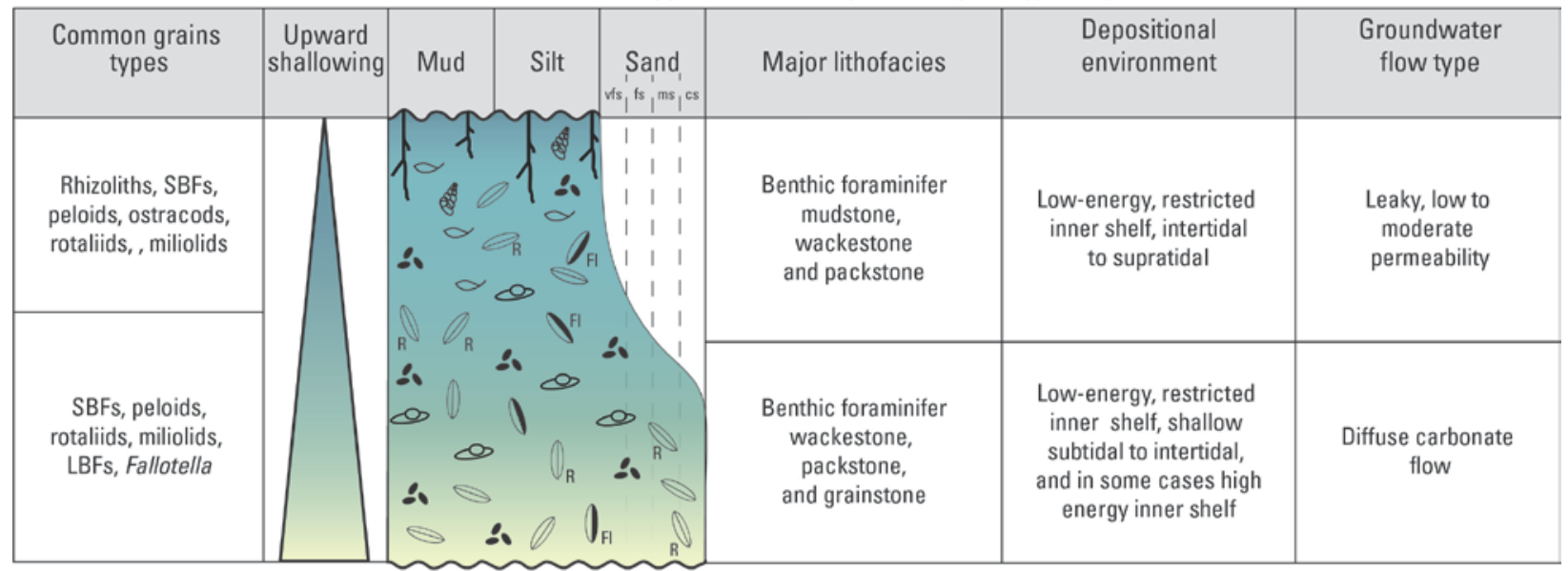

\section{EXPLANATION}

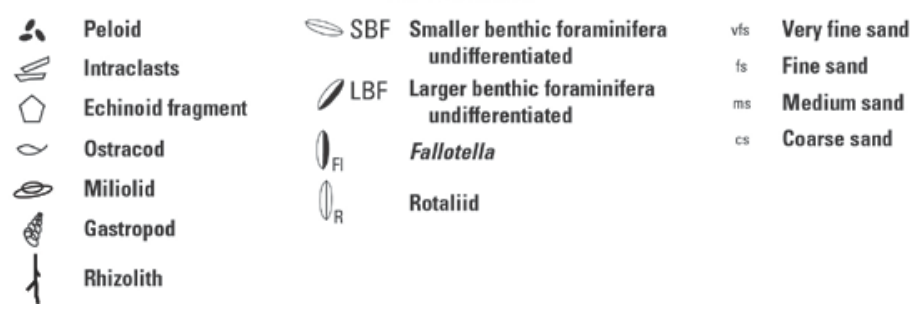

Figure 3. Diagrams showing two of three ideal high-frequency cycles defined for depositional sequence DS AP2 of the Avon Park Formation by analyzing slabbed core from U.S. Geological Survey G-2984 test corehole (figs. 1 and 2). $A$, The type I microbial laminite-capped grain-rich peritidal ideal cycle. $B$, The type II rhizolith- and mud-capped micrite-rich peritidal ideal cycle. 
$\boldsymbol{A}$

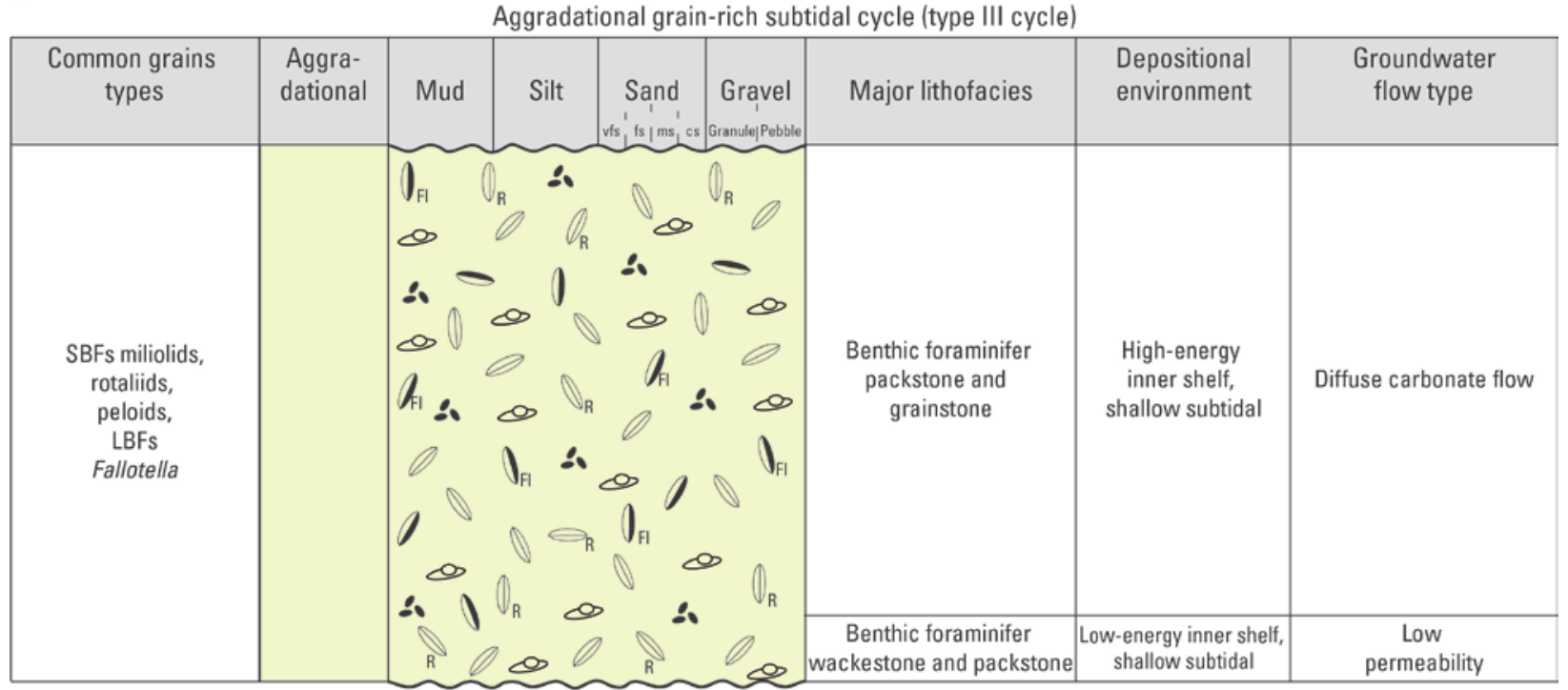

B

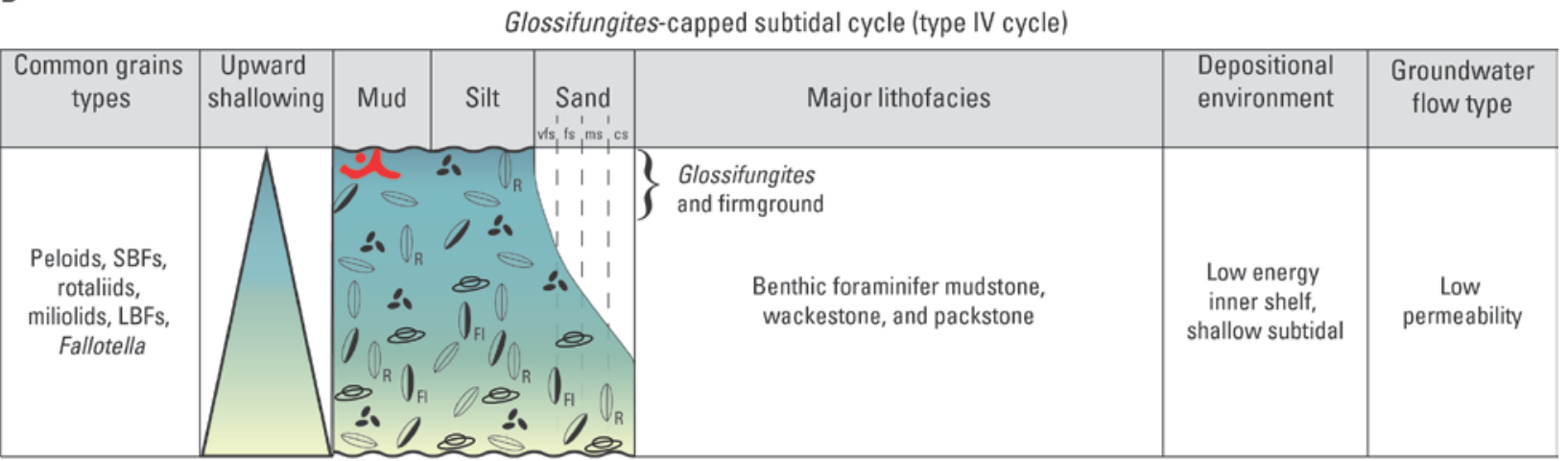

\section{EXPLANATION}

\begin{tabular}{|c|c|c|c|c|c|}
\hline 1 & Glossifungites & $\Leftrightarrow S B F$ & $\begin{array}{l}\text { Smaller benthic foraminifera } \\
\text { undifferentiated }\end{array}$ & vis & $\begin{array}{l}\text { Very fine sand } \\
\text { Fine sand }\end{array}$ \\
\hline \&. & Peloid & LBF & Larger benthic foraminifera & $\mathrm{ms}$ & Medium sand \\
\hline$\theta$ & Miliolid & & undifferentiated & cs & Coarse sand \\
\hline & & & Rotaliid & & \\
\hline
\end{tabular}

Figure 4. Diagrams showing two of four ideal high-frequency cycles defined for depositional sequence DS AP2 and DS AP3 of the Avon Park Formation by analyzing slabbed core from U.S. Geological Survey G-2984 test corehole (figs. 1 and 2). A, The type III aggradational grain-rich subtidal ideal cycle, which is exclusive to the depositional sequence AP3 of the Avon Park Formation. B, The type IV Glossifungitescapped ideal cycle. 


\section{References}

Budd, D.A., Saller, A.J., and Harris, P.M., (eds.), 1995, Unconformities and porosity in carbonate strata: American Association of Petroleum Geologists Memoir 63, 313 p.

Cander, Harris, 1995, Interplay of water-rock interaction efficiency, unconformities, and fluid flow in a carbonate aquifer: Floridan aquifer system, in Budd, D.A., Saller, A.H., and Harris, P.M., eds., Unconformities and porosity in carbonate strata: American Association of Petroleum Geologists Memoir 63, p. 103-124.

Choquette, P.W., and Pray, L.C., 1970, Geologic nomenclature and classification of porosity in sedimentary carbonates: American Association of Petroleum Geologists Bulletin, v. 54, p. 207-250.

Cohen, K.M., Finney, S.C., Gibbard, P.L., and Fan, J.-X., 2013 updated, The ICS international chronostratigraphic chart: Episodes, v. 36, p. 199-204.

Cunningham, K.J., and Sukop, M.C., 2011, Multiple technologies applied to characterization of the porosity and permeability of the Biscayne aquifer, Florida: U.S. Geological Survey OpenFile Report 2011-1037, 8 p.

Cunningham, K.J., and Sukop, M.C., 2012, Megaporosity and permeability of Thalassinoidesdominated ichnofabrics in the Cretaceous carbonate Edwards-Trinity aquifer system, Texas: U.S. Geological Survey Open-File Report 2011-1021, 4 p.

Cunningham, K.J., Sukop, M.C., and Curran, H.A., 2012, Chapter 28-Carbonate aquifers, in Knaust, D., and Bromley, R.G., Developments in Sedimentology, v. 26: Elsevier, New York, p. 869-896.

Cunningham, K.J., Sukop, M.C., Huang, H., Alvarez, P.F., Curran, H.A., Renken, R.A., and Dixon, J.F., 2009, Prominence of ichnologically influenced macroporosity in the karst Biscayne aquifer-Stratiform "super-K" zones: Geological Society of America Bulletin, v. 121, no. 1-2, p. 164-180.

Cunningham, K.J., Wacker, M.A., Robinson, Edward, Dixon, J.F., and Wingard, G.L., 2006, A Cyclostratigraphic and Borehole-Geophysical Approach to Development of a ThreeDimensional Conceptual Hydrogeologic Model of the Karstic Biscayne Aquifer, Southeastern Florida: U.S. Geological Survey Scientific Investigations Report 2005-5235, 69 p., plus CD.

Droser, M.L., and Bottjer, D.J., 1986, A semiquantitative field classification of ichnofabric: Journal of Sedimentary Petrology, v. 56, p. 558-559.

Droser, M.L., and Bottjer, D.J., 1989, Ichnofabric of sandstones deposited in high-energy nearshore environments-Measurement and utilization: Palaios, v. 4, p. 598-604.

Dunham, R.J., 1962, Classification of carbonate rocks according to depositional textures, in Ham, W.E., ed., Classification of carbonate rocks: American Association of Petroleum Geologists, Memoir 1, p. 108-121.

Embry, A.F., and Klovan, J.E., 1971, A late Devonian reef tract on Northeastern Banks Island, N.W.T.: Bulletin of Canadian Petroleum Geology, v. 19, p. 730-781.

Flügel, Erik, 2004, Microfacies of carbonate rocks. Analysis, interpretation and application: New York, Springer, 976 p.

Geological Society of America, 1991, Rock color chart: Baltimore, Md., Munsell Color.

Haq, B.U., Hardenbol, J., and Vail, P.R., 1987, Chronology of fluctuating sea levels since the Triassic (250 million years ago to present): Science, v. 235, p. 1156-1167.

Kerans, Charles, and Tinker, S.W., 1997, Sequence stratigraphy and characterization of carbonate reservoirs: Society of Economic Paleontologists and Mineralogists, Short Course Notes 40, 130 p. 
Lucia, F.J., 1995, Rock-fabric/petrophysical classification of carbonate pore space for reservoir characterization: American Association of Petroleum Geologists Bulletin, v. 79, p. 1275-1300. Lucia, F.J., 1999, Carbonate reservoir characterization: Berlin, Springer-Verlag, 226 p.

Neuendorf, K.K.E.; Mehl, J.P., Jr.; and Jackson, J.A., (eds.), 2005, Glossary of geology (5th ed.): American Geological Institute, Alexandria, Va., 769 p.

Olsson, A.A., and Petit, R.E., 1964, Some Neogene Mollusca from Florida and the Carolinas:

Bulletins of American Paleontology, v. 47, no. 217, p. 507-584.

Pettijohn, F.J., 1957, Sedimentary rocks: New York, Harper, 779 p.

Reese, R.S., and Cunningham, K.J., 2014, Hydrogeologic framework and salinity distribution of the Floridan aquifer system of Broward County, Florida: U.S. Geological Survey Scientific Investigations Report 2014-5029, 60 p.

Stenzel, H.B., 1971, Oysters, in Moore, R.C., ed., Treatise on invertebrate paleontology, part N, v. 3, Mollusca 6, University of Kansas and Geological Society of America: Lawrence, Kans., p. N953-N1224.

Sukop, M.C., and Cunningham, K.J., 2014, Lattice Boltzmann methods applied to large-scale three-dimensional virtual cores constructed from digital optical borehole images of the karst carbonate Biscayne aquifer in southeastern Florida: Water Resources Research, v. 50, p. 88078825, https://doi.org/10.1002/2014WR015465.

Sukop, M.C., Huang, H., Alvarez, P.F., Variano, E.A., and Cunningham, K.J., 2013, Evaluation of permeability and non-Darcy flow in vuggy macroporous limestone aquifer samples with lattice Boltzmann methods: Water Resources Research, v. 49, issue 1, p. 216-230, https://doi.org/10.1029/2011WR011788.

Ward, L.W., 1992, Molluscan biostratigraphy of the Miocene, middle Atlantic Coastal plain of North America: Virginia Museum of Natural History Memoir, no. 2, 145 p.

Wright, V.P., and Tucker, M.E., 1991, Calcretes: an introduction, in Wright, V.P., and Tucker, M.E., eds., Calcretes: Blackwell Scientific Publications, Boston, p. 1-22.

Woodring, W.P., 1982, Geology and paleontology of Canal Zone and adjoining parts of Panama-Description of Tertiary mollusks (pelecypods-Propeamussiidae to Cuspidariidae; additions to families covered in P 306-E; additions to gastropods; cephalopods): U.S. Geological Survey Professional Paper 306-F, p. 541-759. 


\section{Lithofacies Description and Sequence Stratigraphy of Continuously Drilled Samples from the Avon Park Formation at U.S. Geological Survey G-2984 Test Corehole}

\begin{tabular}{|c|c|}
\hline $\begin{array}{l}\text { Depth } \\
\text { interval } \\
\text { (feet bls) }\end{array}$ & $\begin{array}{l}\text { Estimates of permeability: Based on comparison of Avon Park Formation lithofacies } \\
\text { and pore types to } 276 \text { Pliocene and Pleistocene eogenetic carbonate rock specimens with } \\
\text { similar lithofacies and pore types and their air-permeability measurements (Cunningham } \\
\text { and others, 2006) and lattice Boltzmann permeability calculations of both Pleistocene } \\
\text { and Cretaceous carbonate rocks (Cunningham and others, 2009, 2012; Cunningham and } \\
\text { Sukop, 2011, 2012; Sukop and others, 2013; Sukop and Cunningham, 2014) } \\
\text { Colors: Colors based on comparison to Munsell rock color chart (Geological Society of } \\
\text { America, 1991) } \\
\text { Ichnofabric: Index based on Droser and Bottjer (1986, 1989) } \\
\text { Top of Avon Park Formation: } 1,067.60 \mathrm{ft} \text { bls (obi depth) and 1,066.0 ft bls (driller's } \\
\text { depth) }\end{array}$ \\
\hline $\begin{array}{l}\text { obi } \\
\text { depth: } \\
1,067.60- \\
1,068.30 \\
\text { ft bls } \\
\text { Driller's } \\
\text { depth: } \\
1,066.0- \\
1,066.7 \mathrm{ft} \\
\text { bls }\end{array}$ & $\begin{array}{l}\text { Cycle type: Top type III cycle and depositional sequence AP3 } \\
\text { Lithofacies: Benthic foraminifer packstone and grainstone } \\
\text { Depositional texture: Smaller and larger benthic foraminifera mud- and grain- } \\
\text { dominated packstone } \\
\text { Color: Very pale orange } 10 \text { YR } 8 / 2 \\
\text { Sedimentary structures: Thickly bedded } \\
\text { Trace fossils: Bioturbated } \\
\text { Ichnofabrics: Ichnofabric index } 5 \\
\text { Carbonate grains: Smaller benthic foraminifera (including smaller miliolids, rotaliids), } \\
\text { peloids, larger benthic foraminifera (larger miliolids, Fallotella floridana), intraclasts, } \\
\text { echinoid plates, ostracods, uncommon thin disarticulated bivalves. Foraminifera } \\
\text { observed in thin section G2984-1066.05 include Fallotella floridana, } \\
\text { Pseudochrysalidina floridana, larger valvulinids, total of } 12 \text { conical larger benthic } \\
\text { foraminifera } \\
\text { Porosity and permeability: } 5-15 \text { percent interparticle and intraparticle porosity, 1-3 } \\
\text { percent particle moldic porosity; } 6 \text {-18 percent total porosity and relatively low } \\
\text { permeability } \\
\text { Depositional environment: High-energy inner platform, shallow subtidal } \\
\text { Comments: Evidence for karstic exposure surface includes core with vugs infilled with } \\
\text { sediment from the Arcadia Formation to a depth of } 3.4 \text { ft below the upper bounding } \\
\text { surface. Some of the vugs are vertically oriented and circular pipe-shaped, and have an } \\
\text { up to 2-centimeter (cm) wide inner diameter. Up to } 0.3 \mathrm{ft} \text { of karstic microrelief on upper } \\
\text { bounding surface, including some cracking extending downward from the upper surface } \\
\text { (as seen on the optical borehole wall image) } \\
\text { Thin section: G2984-1066.05 }\end{array}$ \\
\hline
\end{tabular}




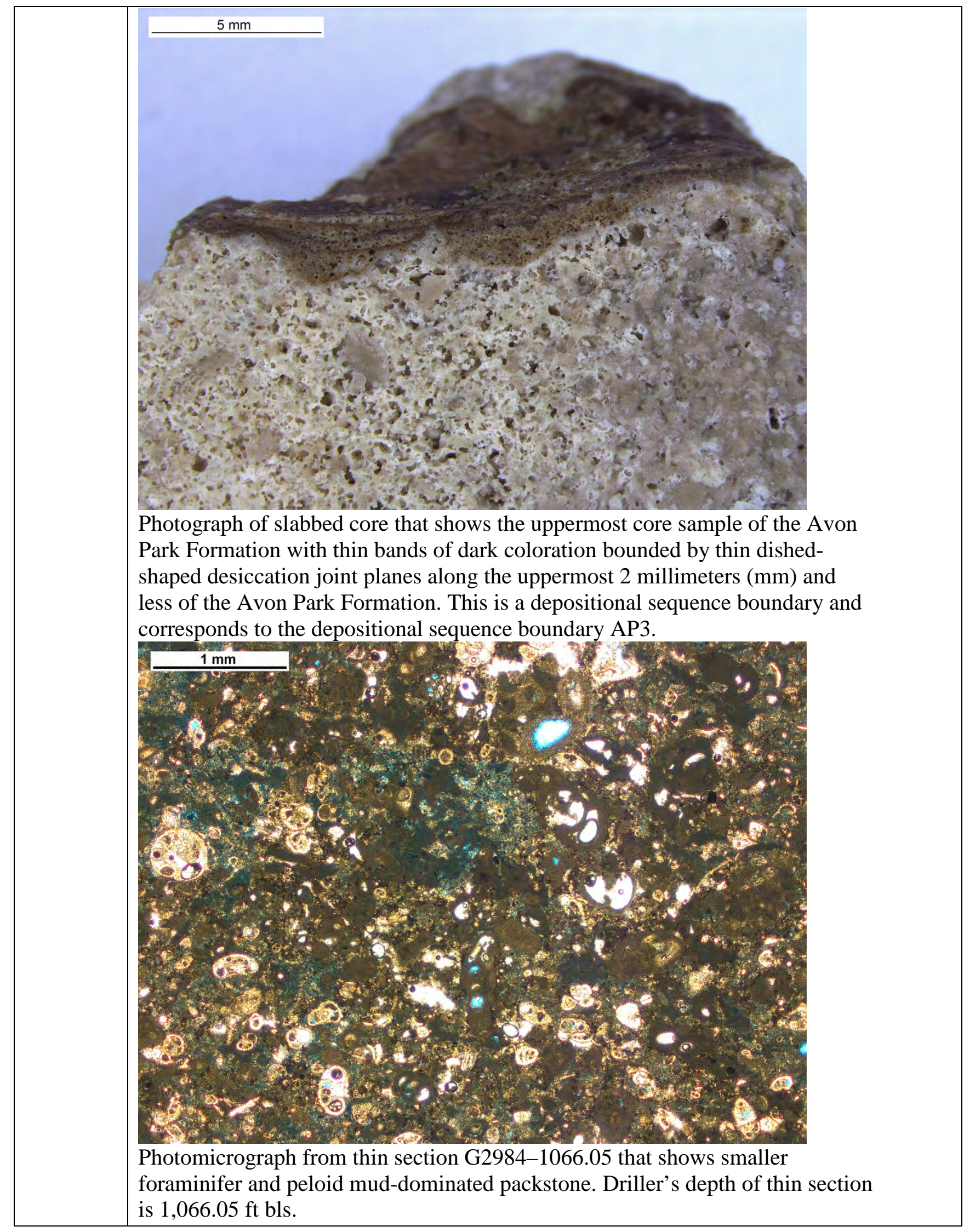




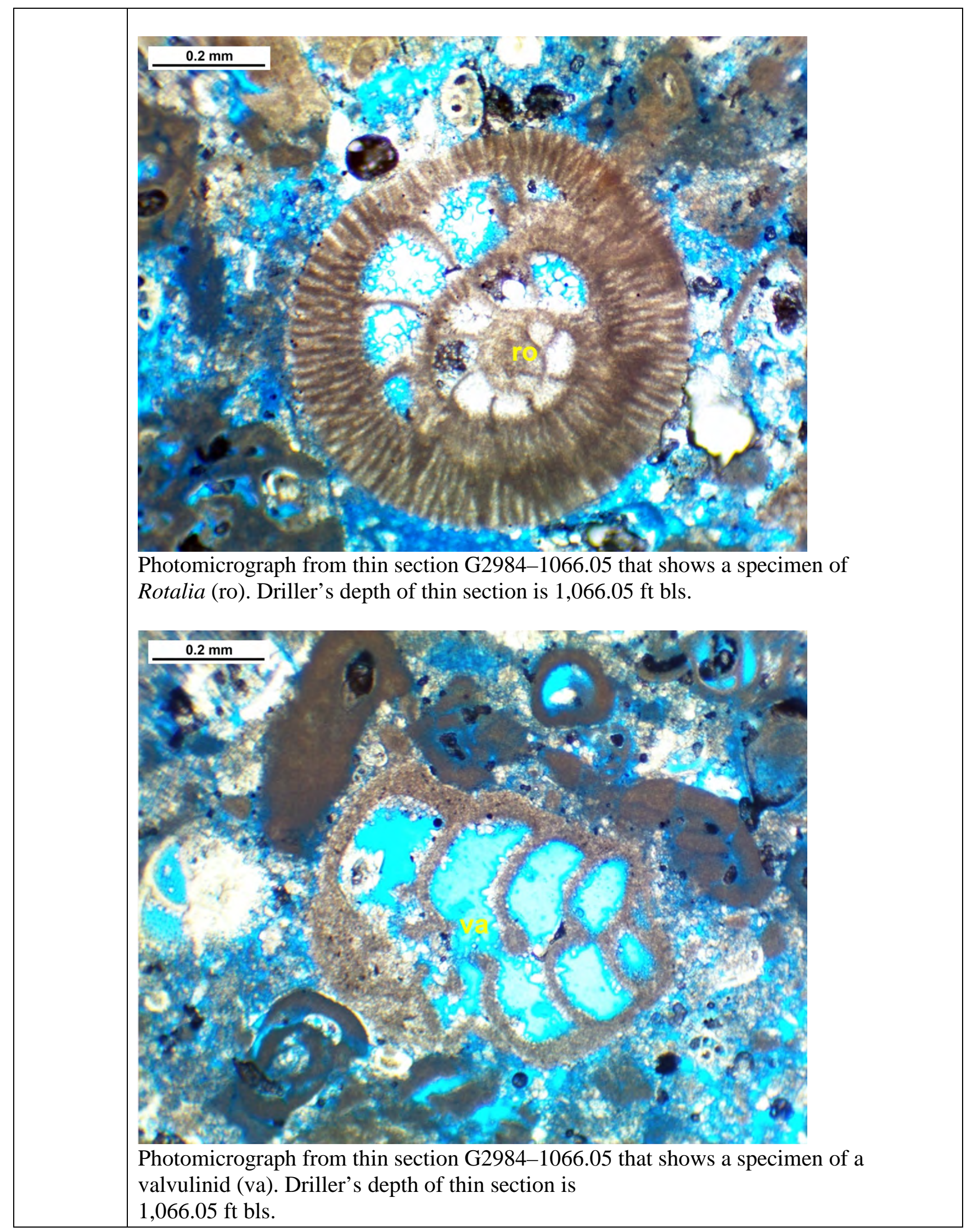




\begin{tabular}{|c|c|}
\hline $\begin{array}{l}\text { obi } \\
\text { depth: } \\
\text { 1,068.30- } \\
1,103.7 \mathrm{ft} \\
\text { bls } \\
\text { Driller's } \\
\text { depth: } \\
\text { 1,066.7- } \\
\text { 1,099.8 ft } \\
\text { bls }\end{array}$ & 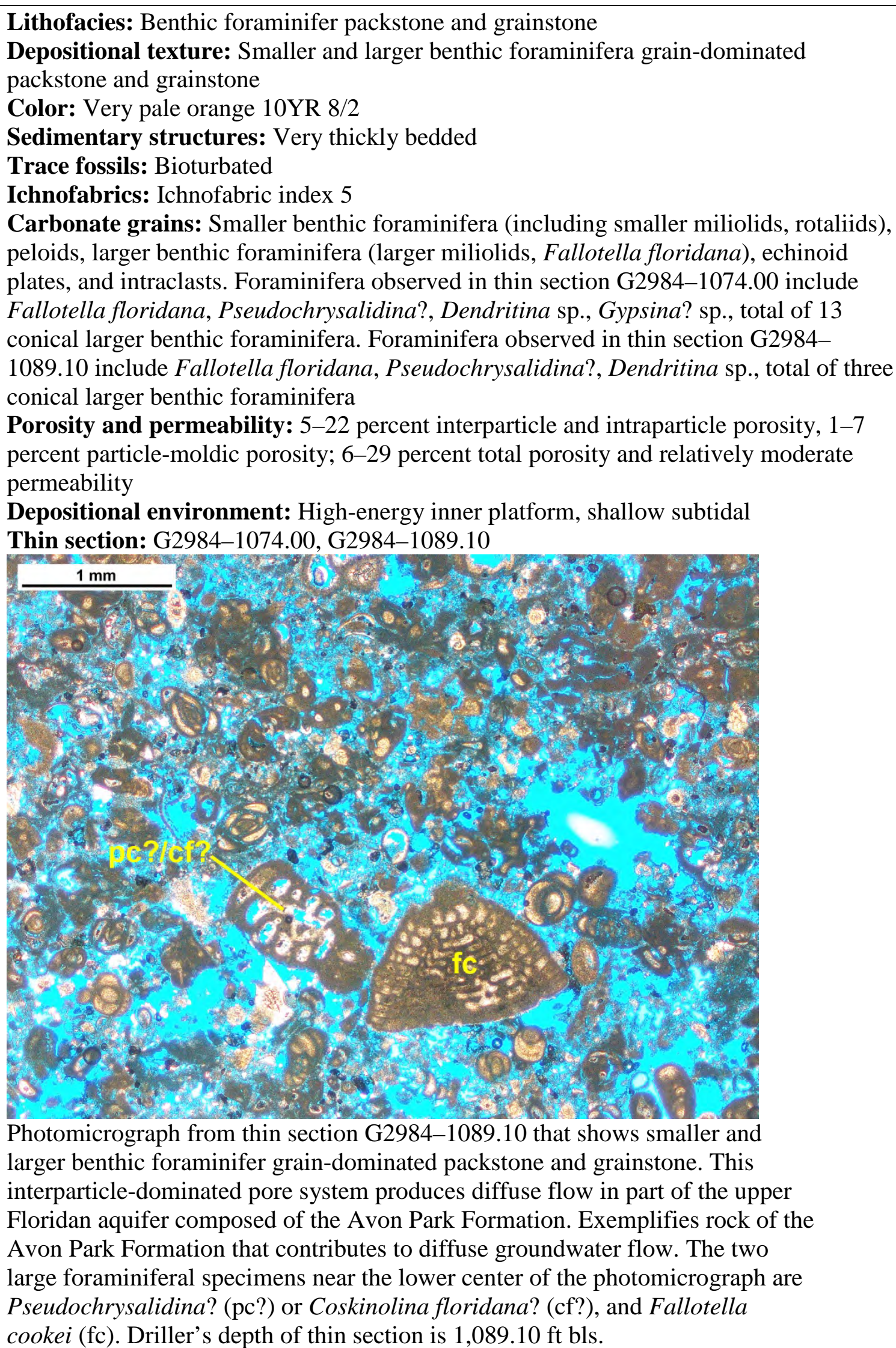 \\
\hline
\end{tabular}




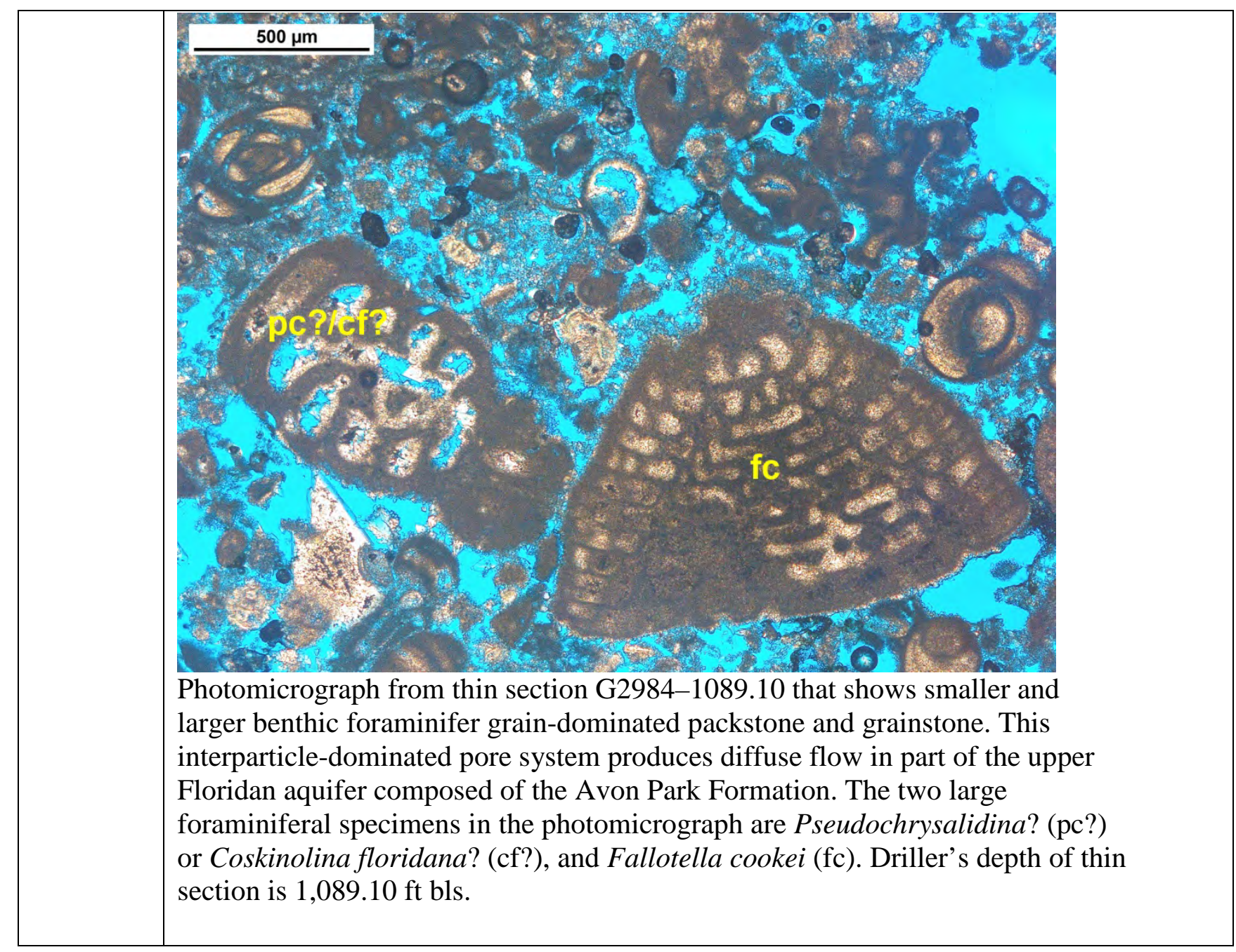




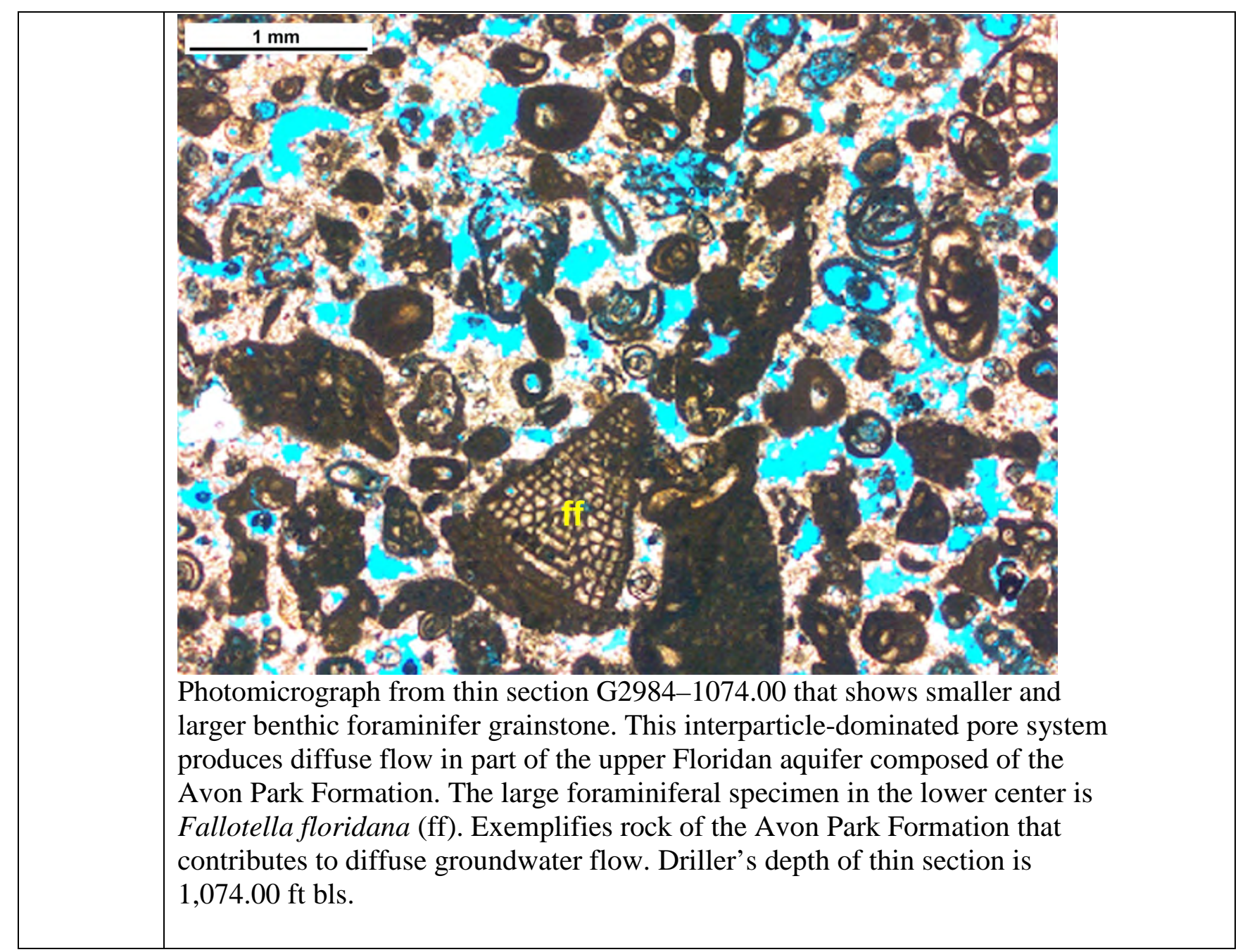




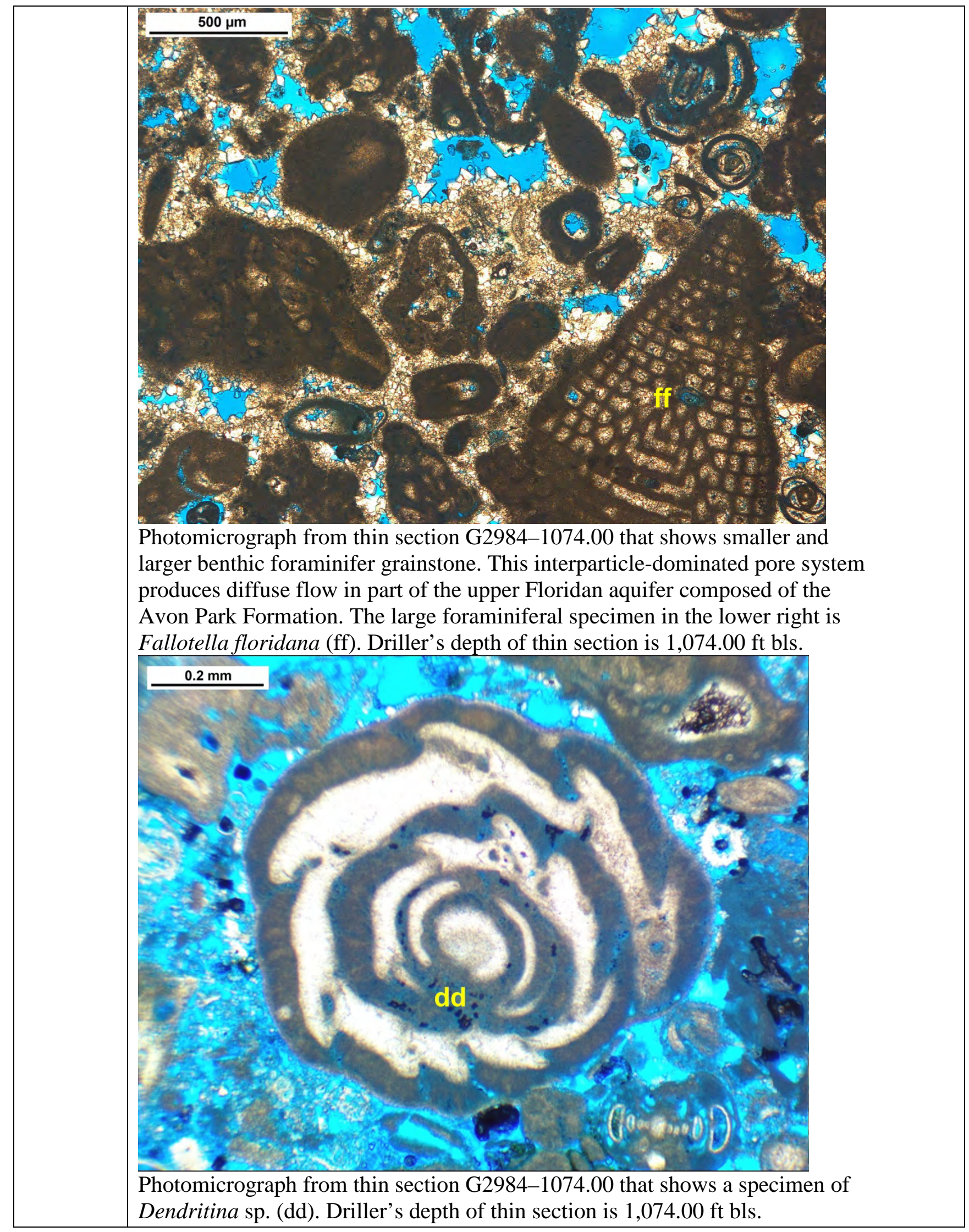




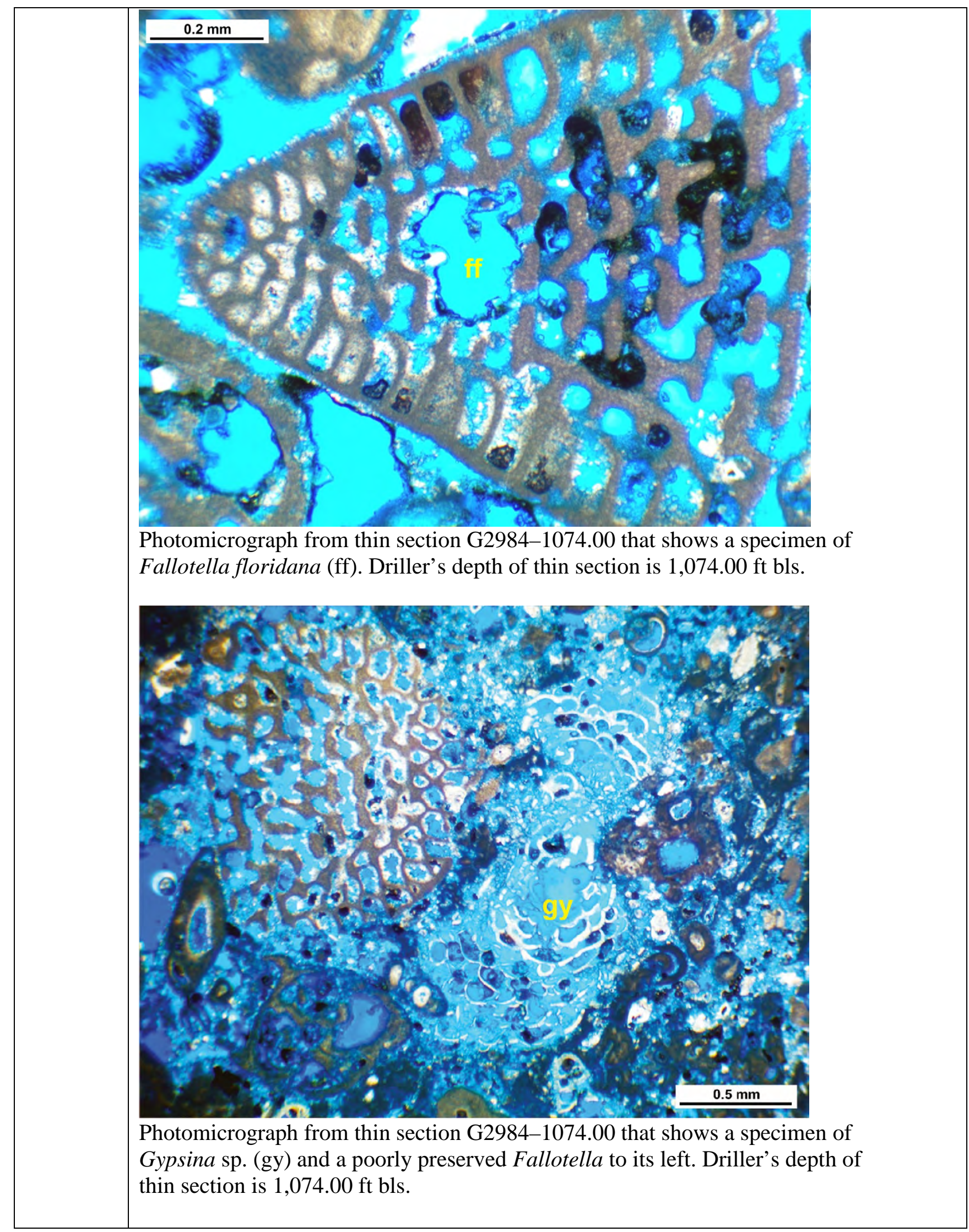




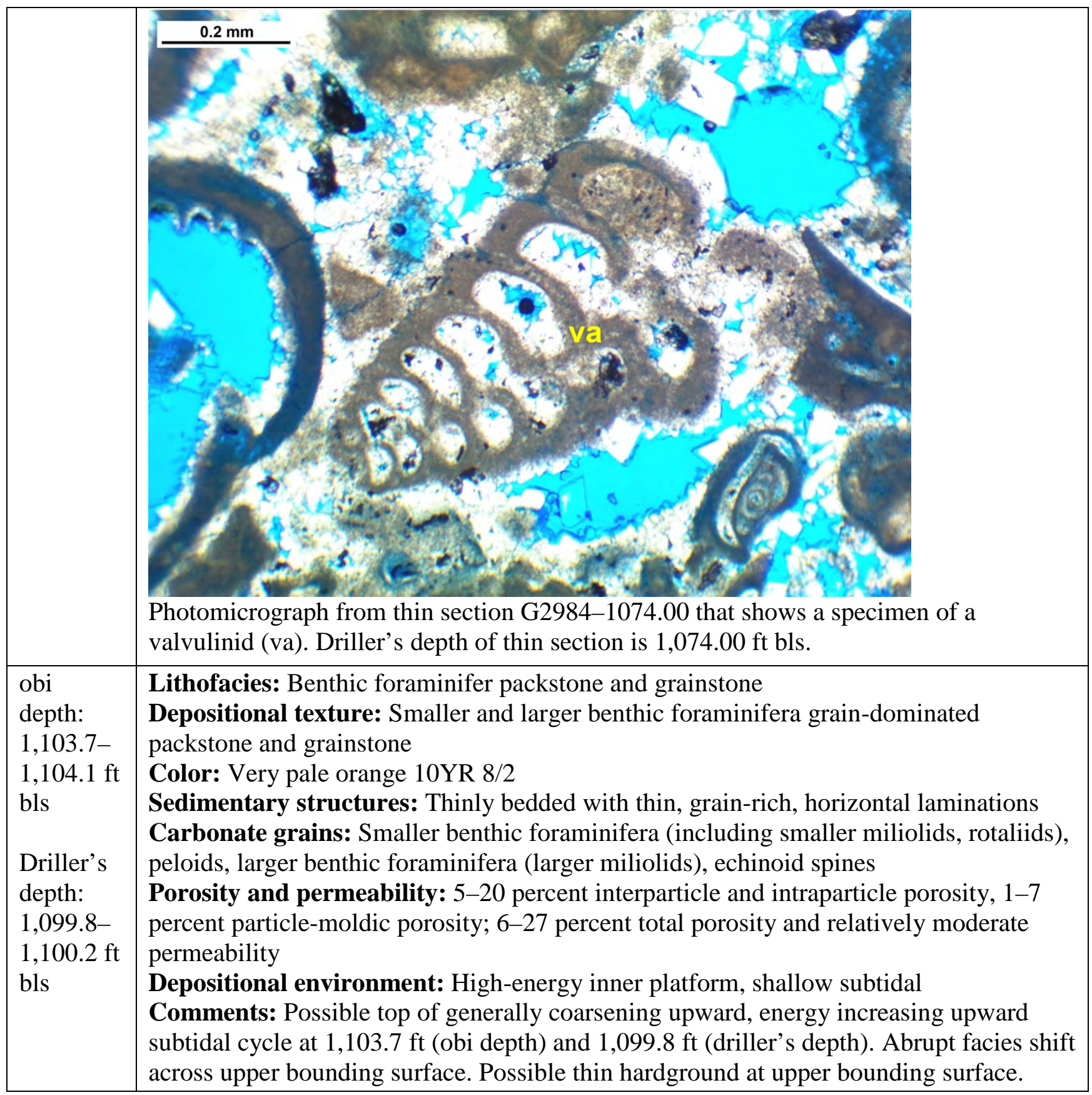




\begin{tabular}{|c|c|}
\hline & 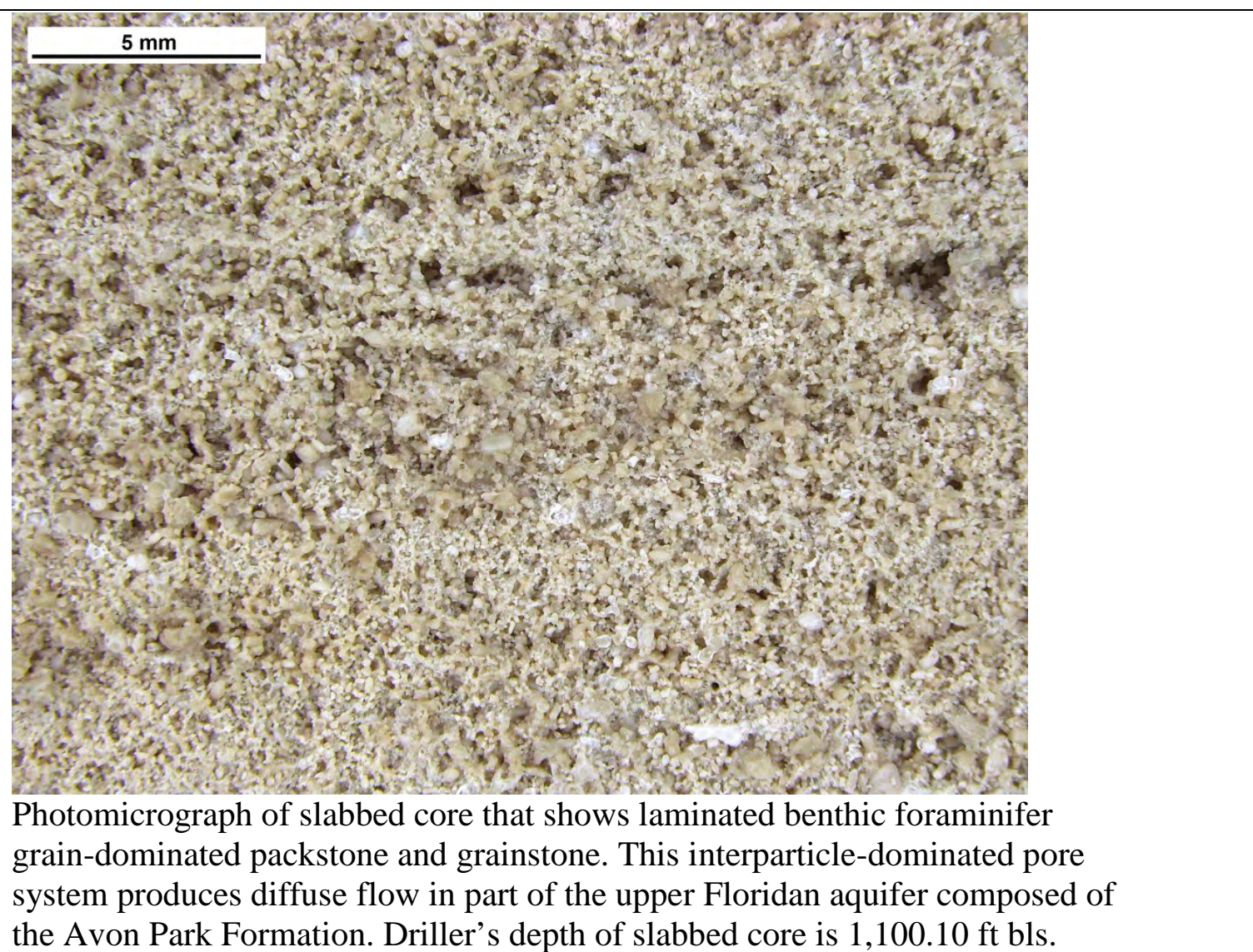 \\
\hline $\begin{array}{l}\text { obi } \\
\text { depth: } \\
1,104.1- \\
1,108.9 \mathrm{ft} \\
\text { bls } \\
\text { Driller's } \\
\text { depth: } \\
\text { 1,100.2- } \\
\text { 1,106.0 ft } \\
\text { bls }\end{array}$ & $\begin{array}{l}\text { Lithofacies: Benthic foraminifer packstone and grainstone } \\
\text { Depositional texture: Smaller and larger benthic foraminifer mud- and grain-dominated } \\
\text { packstone and grainstone } \\
\text { Color: Very pale orange } 10 \text { YR } 8 / 2 \\
\text { Trace fossils: Bioturbated } \\
\text { Ichnofabrics: Ichnofabric index } 5 \\
\text { Sedimentary structures: Very thickly bedded } \\
\text { Carbonate grains: Smaller benthic foraminifera (including smaller miliolids, rotaliids), } \\
\text { peloids, larger benthic foraminifera (Fallotella, larger miliolids), echinoid spines } \\
\text { Porosity and permeability: 5-18 percent interparticle and intraparticle porosity, 1-3 } \\
\text { percent particle-moldic porosity; } 6 \text {-21 percent total porosity and relatively moderate } \\
\text { permeability } \\
\text { Depositional environment: High-energy inner platform, shallow subtidal }\end{array}$ \\
\hline $\begin{array}{l}\text { obi } \\
\text { depth: } \\
1,108.9- \\
1,110.9 \mathrm{ft} \\
\text { bls } \\
\text { Driller's } \\
\text { depth: } \\
1,106.0-\end{array}$ & $\begin{array}{l}\text { Lithofacies: Benthic foraminifer wackestone and packstone } \\
\text { Depositional texture: Smaller and larger benthic foraminifer mud-dominated packstone } \\
\text { Color: Very pale orange 10YR 8/2 } \\
\text { Sedimentary structures: Thickly bedded } \\
\text { Trace fossils: Bioturbated } \\
\text { Ichnofabrics: Ichnofabric index } 5 \\
\text { Carbonate grains: Smaller benthic foraminifera (including smaller miliolids, rotaliids), } \\
\text { peloids, larger benthic foraminifera (Fallotella, larger miliolids), echinoid spines } \\
\text { Porosity and permeability: 5-10 percent interparticle and intraparticle porosity, 1-3 }\end{array}$ \\
\hline
\end{tabular}




\begin{tabular}{|c|c|}
\hline $\begin{array}{l}1,108.0 \mathrm{ft} \\
\text { bls }\end{array}$ & $\begin{array}{l}\text { percent particle-moldic porosity; 6-13 percent total porosity and relatively low } \\
\text { permeability } \\
\text { Depositional environment: Low-energy inner platform, shallow subtidal }\end{array}$ \\
\hline $\begin{array}{l}\text { obi } \\
\text { depth: } \\
\text { 1,110.9- } \\
\text { 1,111.4 ft } \\
\text { bls } \\
\text { Driller's } \\
\text { depth: } \\
\text { 1,108.0- } \\
\text { 1,109.5 ft } \\
\text { bls }\end{array}$ & 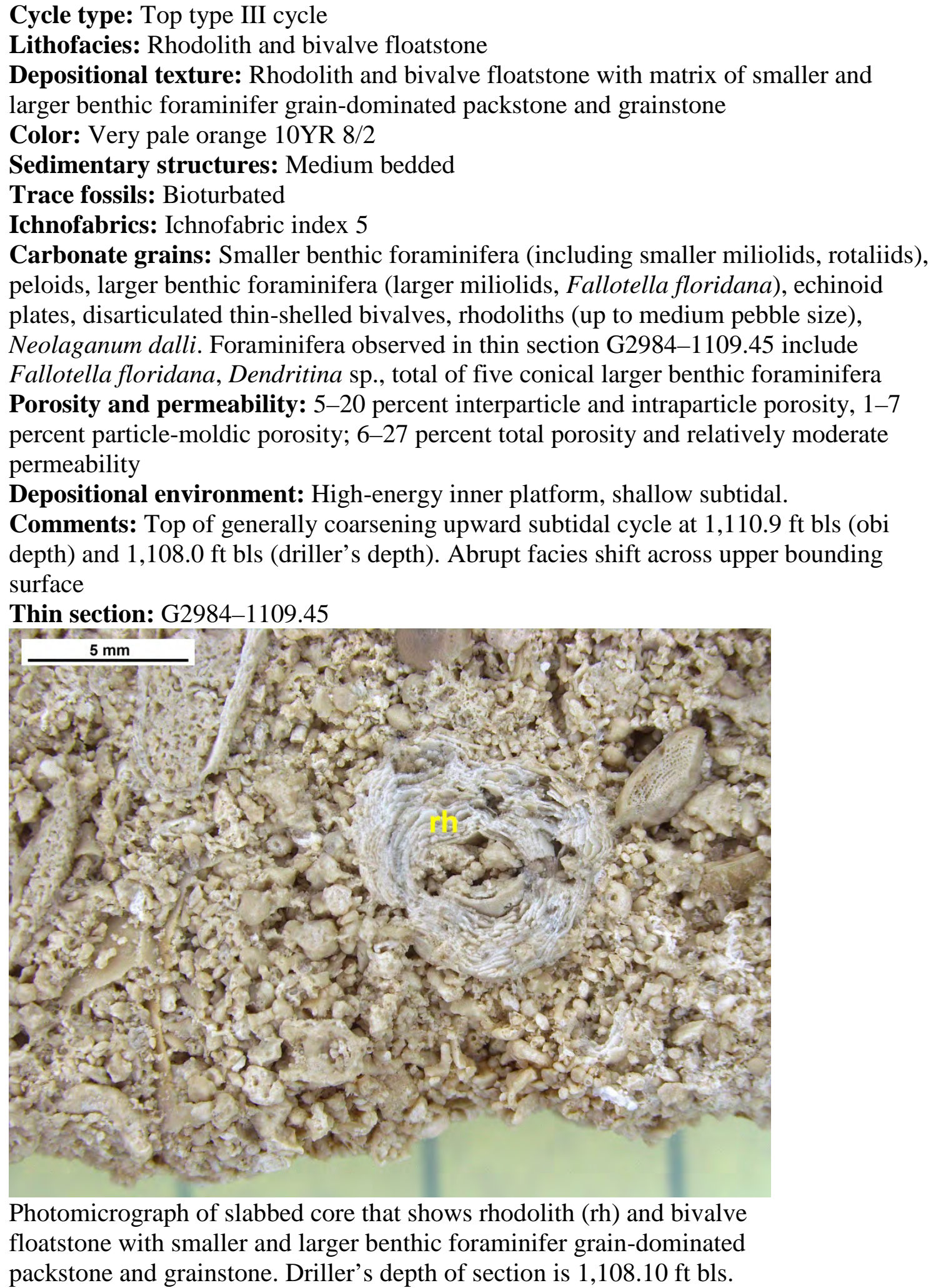 \\
\hline
\end{tabular}




\begin{tabular}{|c|c|}
\hline & 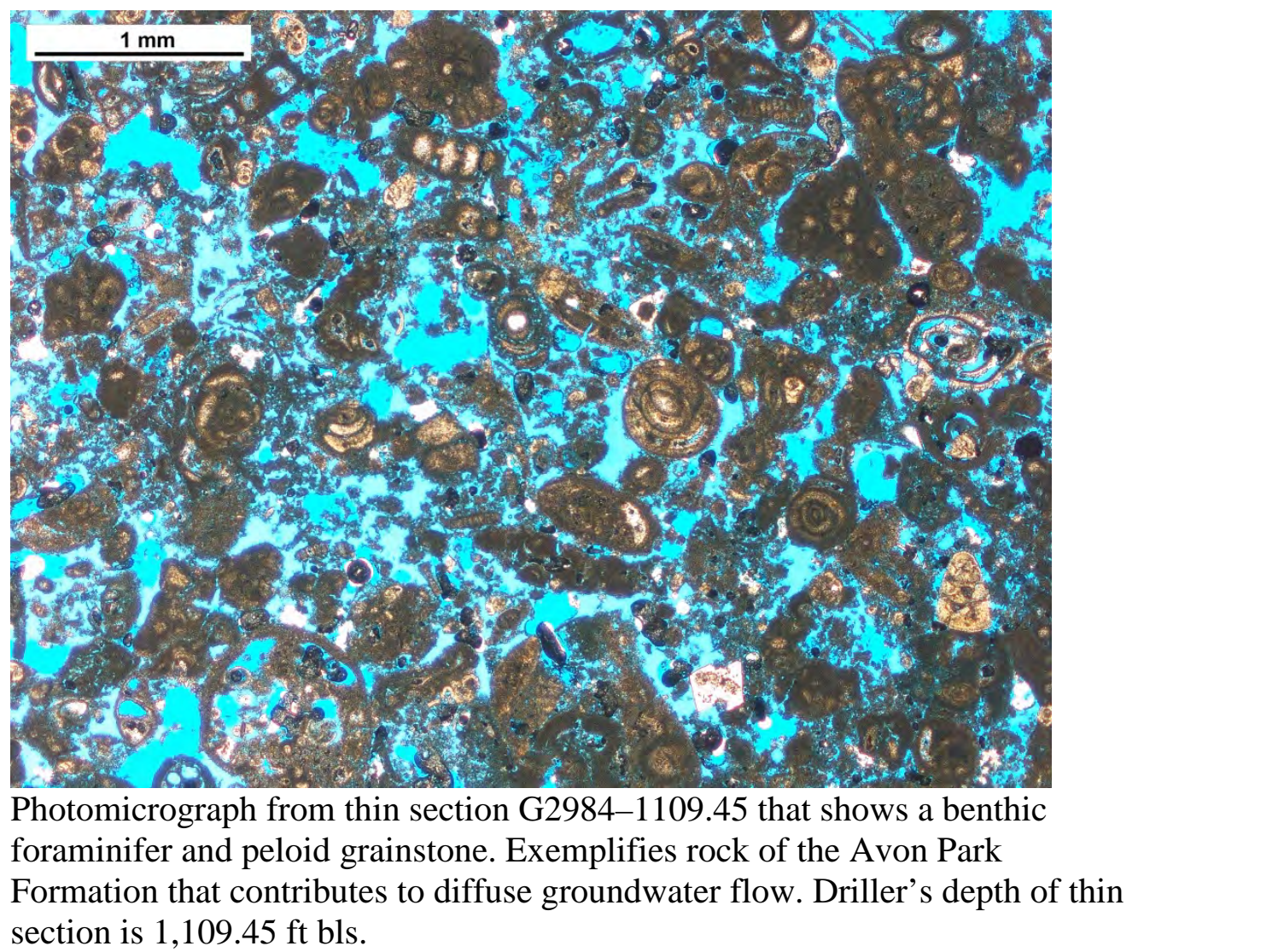 \\
\hline $\begin{array}{l}\text { obi } \\
\text { depth: } \\
1,111.4- \\
1,121.6 \mathrm{ft} \\
\text { bls } \\
\\
\text { Driller's } \\
\text { depth: } \\
\text { 1,109.5- } \\
\text { 1,119.8 ft } \\
\text { bls }\end{array}$ & $\begin{array}{l}\text { Cycle type: Intra-type III cycle } \\
\text { Lithofacies: Benthic foraminifer packstone and grainstone } \\
\text { Depositional texture: Smaller and larger benthic foraminifer grain-dominated } \\
\text { packstone and grainstone } \\
\text { Color: Very pale orange } 10 \text { YR } 8 / 2 \\
\text { Sedimentary structures: Medium bedded } \\
\text { Trace fossils: Bioturbated } \\
\text { Ichnofabrics: Ichnofabric index } 5 \\
\text { Carbonate grains: Smaller benthic foraminifera (including smaller miliolids, rotaliids), } \\
\text { peloids, larger benthic foraminifera (larger miliolids, Fallotella floridana), echinoid } \\
\text { plates and spines } \\
\text { Porosity and permeability: } 5-20 \text { percent interparticle and intraparticle porosity, } 1-7 \\
\text { percent particle-moldic porosity; } 6-27 \text { percent total porosity and relatively moderate } \\
\text { permeability } \\
\text { Depositional environment: High-energy inner platform, shallow subtidal }\end{array}$ \\
\hline $\begin{array}{l}\text { obi } \\
\text { depth: } \\
1,121.6- \\
1,121.8 \mathrm{ft} \\
\text { bls } \\
\text { Driller's }\end{array}$ & $\begin{array}{l}\text { Cycle type: Top type II cycle } \\
\text { Lithofacies: Benthic foraminifer wackestone and packstone } \\
\text { Depositional texture: Smaller benthic foraminifer wackestone } \\
\text { Color: Very light gray N8 } \\
\text { Sedimentary structures: Thickly bedded, irregular vugs, skew-plane desiccation cracks } \\
\text { Trace fossils: Rhizoliths } \\
\text { Carbonate grains: Smaller benthic foraminifera (including miliolids), ostracods, minor }\end{array}$ \\
\hline
\end{tabular}




\begin{tabular}{|c|c|}
\hline $\begin{array}{l}\text { depth: } \\
\text { 1,119.8- } \\
1,120.20 \\
\text { ft bls }\end{array}$ & $\begin{array}{l}\text { peloids, gastropods, intraclasts } \\
\text { Porosity and permeability: } 1-2 \text { percent intraparticle porosity, } 1-2 \text { percent particle- } \\
\text { moldic porosity, } 1-3 \text { percent vuggy porosity, less than } 1 \text { percent irregular vugs; } 3-7 \\
\text { percent total porosity and relatively low permeability } \\
\text { Depositional environment: Low-energy restricted inner platform, intertidal to } \\
\text { supratidal } \\
\text { Comments: Top of generally fining upward peritidal cycle at } 1,121.6 \mathrm{ft} \text { (obi depth) and } \\
\text { 1,119.8 ft (driller's depth). Abrupt facies shift across upper bounding surface. Subaerial } \\
\text { exposure post deposition, as evidenced by karstic enlargement of rhizolith diameters and } \\
\text { irregular vugs. Irregular vugs infilled with sediment from superjacent cycle } \\
\quad 5 \mathrm{~mm} \\
\end{array}$ \\
\hline $\begin{array}{l}\text { obi } \\
\text { depth: } \\
\text { 1,121.8- } \\
\text { 1,127.0 ft } \\
\text { bls } \\
\text { Driller's } \\
\text { depth: } \\
\text { 1,120.2- } \\
\text { 1,126.0 ft } \\
\text { bls }\end{array}$ & $\begin{array}{l}\text { Lithofacies: Benthic foraminifer packstone and grainstone } \\
\text { Depositional texture: Smaller and larger benthic foraminifer grain-dominated } \\
\text { packstone and grainstone } \\
\text { Color: Very pale orange } 10 \text { YR } 8 / 2 \\
\text { Sedimentary structures: Very thickly bedded } \\
\text { Trace fossils: Bioturbated } \\
\text { Ichnofabrics: Ichnofabric index } 5 \\
\text { Carbonate grains: Mainly smaller benthic foraminifer (including rotaliids, miliolids), } \\
\text { peloids, larger benthic foraminifera (including Fallotella floridana, miliolids), minor } \\
\text { amphisteginids (including Amphistegina parvula), echinoid plates and spines. } \\
\text { Foraminifera observed in thin section G2984-1123.50 include smaller benthic } \\
\text { foraminifera, Fallotella floridana, total of } 10 \text { conical larger benthic foraminifera } \\
\text { Porosity and permeability: 1-22 percent interparticle and intraparticle porosity, 1-5 } \\
\text { percent moldic porosity; } 2-27 \text { percent total porosity and moderate permeability }\end{array}$ \\
\hline
\end{tabular}




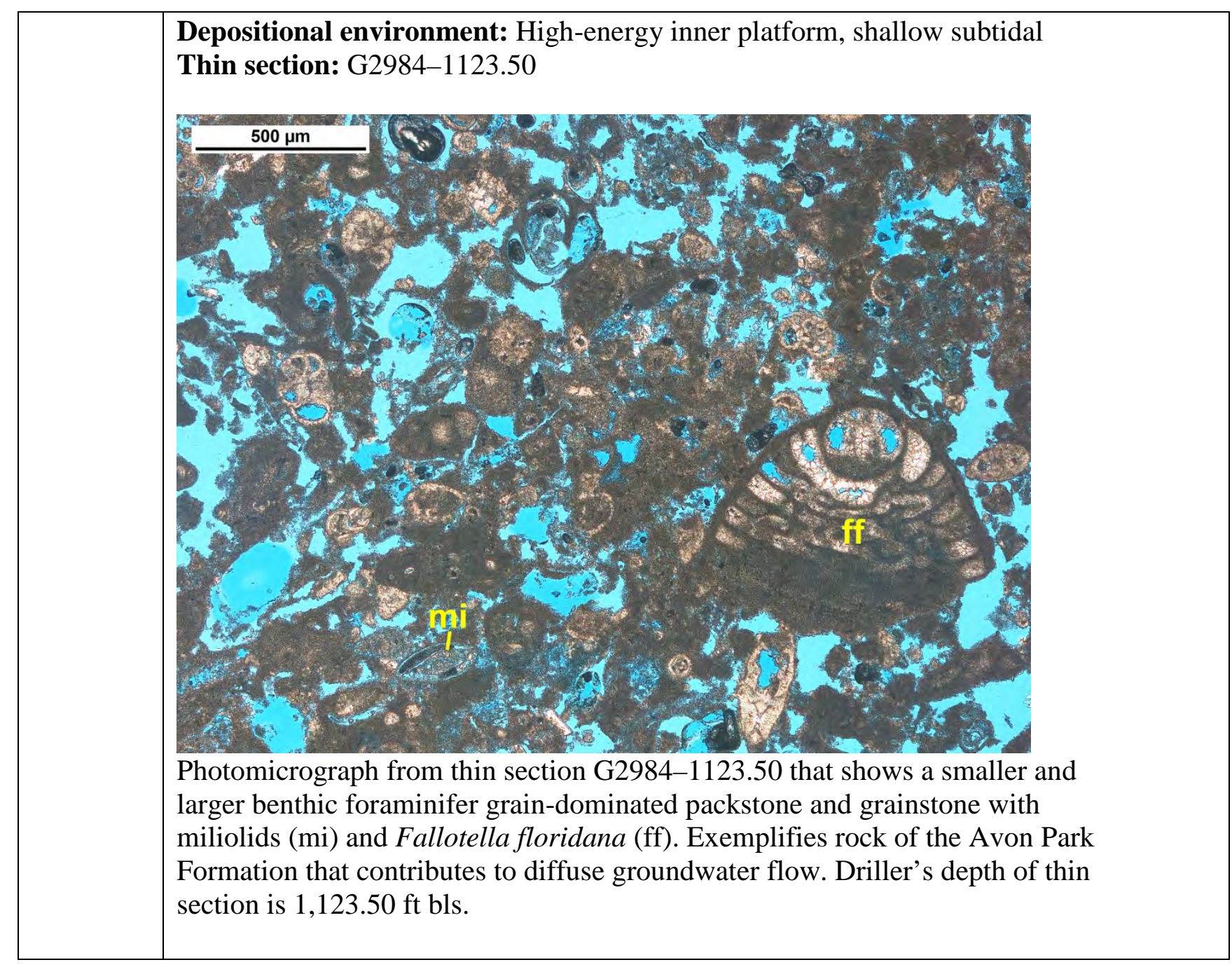




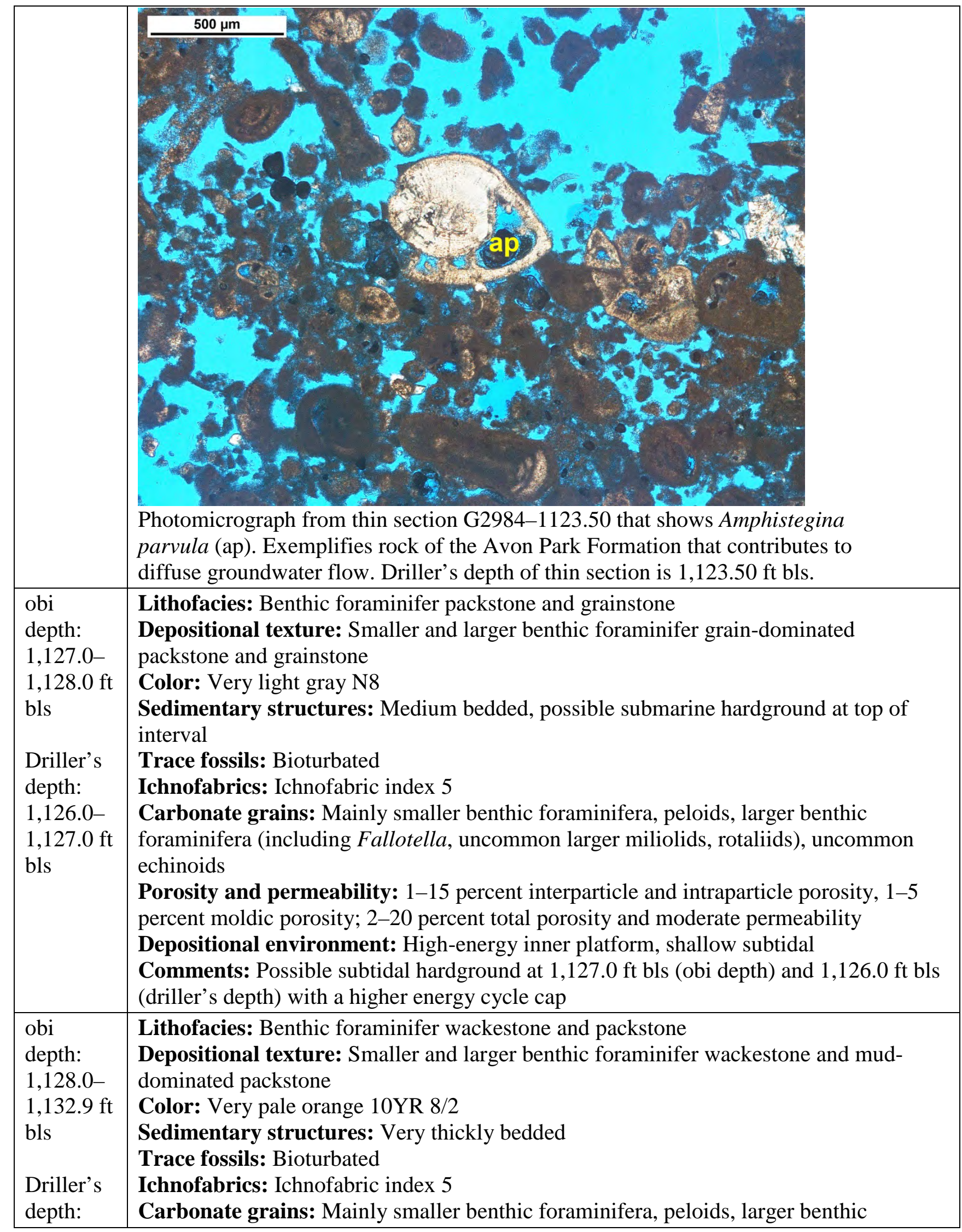




\begin{tabular}{|c|c|}
\hline $\begin{array}{l}1,127.0- \\
1,132.2 \mathrm{ft} \\
\text { bls }\end{array}$ & $\begin{array}{l}\text { foraminifera (including Fallotella floridana, uncommon larger miliolids), uncommon } \\
\text { echinoids } \\
\text { Porosity and permeability: } 1-5 \text { percent intraparticle porosity, } 1-5 \text { percent moldic } \\
\text { porosity; } 2-10 \text { percent total porosity and low permeability } \\
\text { Depositional environment: Low-energy restricted inner platform, shallow subtidal }\end{array}$ \\
\hline $\begin{array}{l}\text { obi } \\
\text { depth: } \\
\text { 1,132.9- } \\
\text { 1,134.2 ft } \\
\text { bls } \\
\text { Driller's } \\
\text { depth: } \\
\text { 1,132.2- } \\
\text { 1,133.5 ft } \\
\text { bls }\end{array}$ & $\begin{array}{l}\text { Lithofacies: Benthic foraminifer wackestone and packstone } \\
\text { Depositional texture: Smaller and larger benthic foraminifer mud-dominated packstone } \\
\text { Color: Very pale orange } 10 \text { YR } 8 / 2 \\
\text { Sedimentary structures: Medium bedded } \\
\text { Trace fossils: Bioturbated } \\
\text { Ichnofabrics: Ichnofabric index } 3-5 \\
\text { Carbonate grains: Mainly smaller benthic foraminifera, peloids, larger benthic } \\
\text { foraminifera (including Fallotella floridana, uncommon larger miliolids), uncommon } \\
\text { echinoids } \\
\text { Porosity and permeability: } 1-5 \text { percent intraparticle porosity, } 1-5 \text { percent moldic } \\
\text { porosity; } 2-10 \text { percent total porosity and low permeability } \\
\text { Depositional environment: Low-energy inner platform, shallow subtidal }\end{array}$ \\
\hline $\begin{array}{l}\text { obi } \\
\text { depth: } \\
\text { 1,134.2- } \\
\text { 1,135.5 ft } \\
\text { bls } \\
\text { Driller's } \\
\text { depth: } \\
\text { 1,133.5- } \\
\text { 1,134.6 ft } \\
\text { bls }\end{array}$ & $\begin{array}{l}\text { Cycle type: Top type II cycle and depositional sequence AP2 } \\
\text { Lithofacies: Benthic foraminifer wackestone and packstone } \\
\text { Depositional texture: Smaller benthic foraminifer wackestone } \\
\text { Color: Yellowish gray } 5 Y \text { Y } 1 \text { (closest to this color when wet) } \\
\text { Sedimentary structures: Thickly bedded, irregular vugs } \\
\text { Trace fossils: Rhizoliths } 0.5-4-m m \text { inner tubule diameter; in some cases, root molds } \\
\text { bifurcate and are solution enlarged, and in some cases lined with micritized grains, } \\
\text { concentric bladed microspar, and concentric micrite } \\
\text { Ichnofabrics: Ichnofabric index } 2-3 \\
\text { Ichnofacies: Psilonichnus } \\
\text { Carbonate grains: Smaller benthic foraminifera (including rotaliids, smaller miliolids), } \\
\text { ostracods, minor peloids, uncommon echinoid spines, abraded (transported) } \\
\text { Microcodium, intraclasts within the uppermost inch of the interval. Foraminifera } \\
\text { observed in thin section G2984-1133.60 include smaller benthic foraminifera, larger } \\
\text { valvulinids, planktic foraminifera } \\
\text { Porosity and permeability: 1-2 percent intraparticle porosity, 3-10 percent root-mold } \\
\text { porosity, 1-7 percent particle-moldic porosity, less than } 1 \text { percent irregular vugs; 4-19 } \\
\text { percent total porosity and relatively low permeability } \\
\text { Depositional environment: Low-energy restricted inner platform, shallow intertidal to } \\
\text { supratidal } \\
\text { Comments: Top of generally fining upward peritidal cycle at 1,134.2 ft bls (obi depth) } \\
\text { and 1,133.5 ft bls (driller's depth). Abrupt facies shift across upper bounding surface. } \\
\text { Subaerial exposure post deposition, as evidenced by karstic enlargement of rhizolith } \\
\text { diameters and irregular vugs, and calcification of rhizoliths (Wright and Tucker, 1991) } \\
\text { Thin section: G2984-1133.60 }\end{array}$ \\
\hline
\end{tabular}




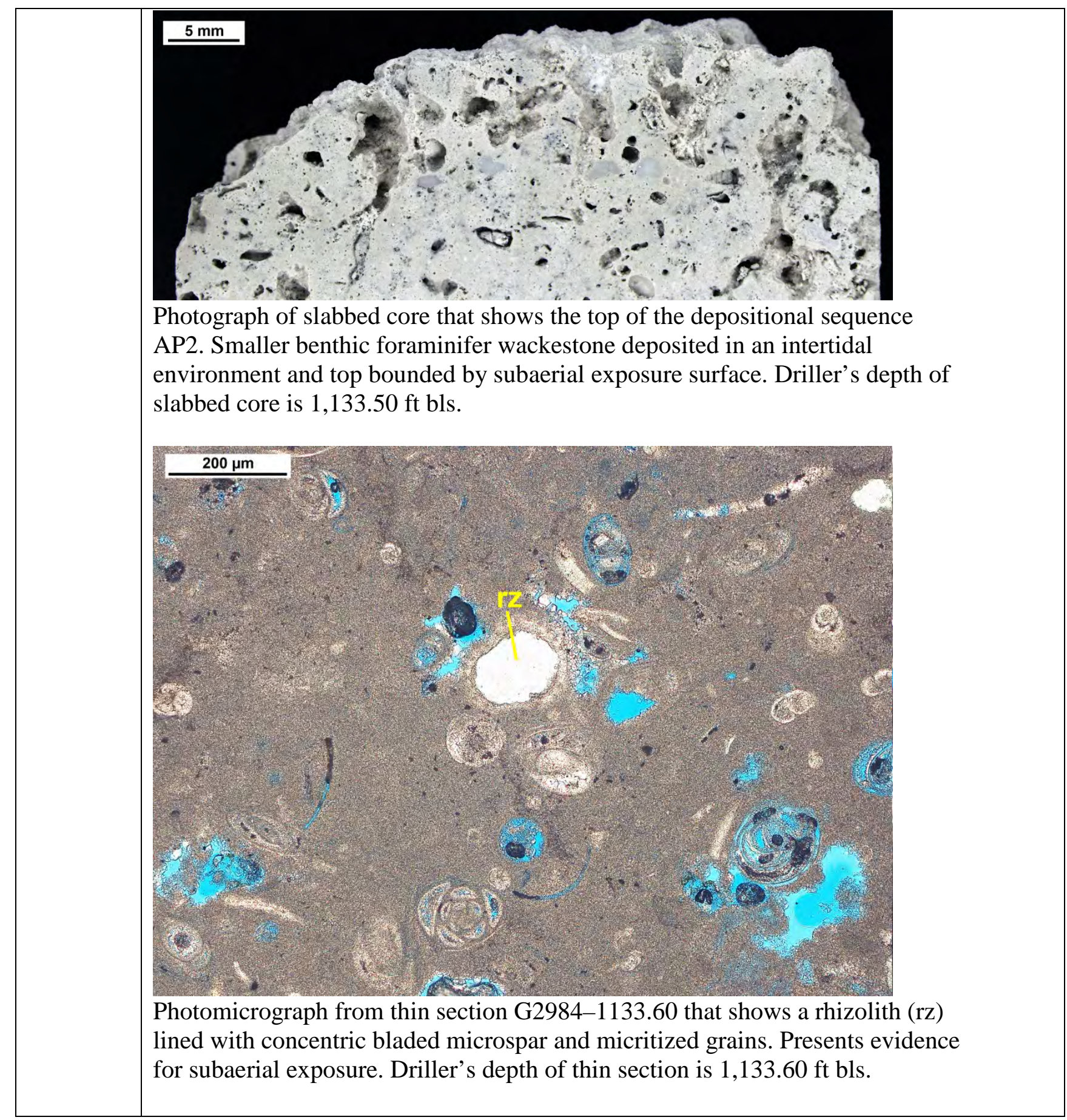




\begin{tabular}{|c|c|}
\hline & $\begin{array}{l}\text { Photomicrograph from thin section G2984-1133.60 that shows rhizolith (rz) } \\
\text { lined with concentric micrite and minor microspar. Presents evidence for } \\
\text { subaerial exposure. Driller's depth of thin section is } 1,133.60 \mathrm{ft} \text { bls. }\end{array}$ \\
\hline $\begin{array}{l}\text { obi } \\
\text { depth: } \\
\text { 1,135.5- } \\
\text { 1,136.3 ft } \\
\text { bls } \\
\text { Driller's } \\
\text { depth: } \\
\text { 1,134.6- } \\
\text { 1,135.4 ft } \\
\text { bls }\end{array}$ & $\begin{array}{l}\text { Lithofacies: Benthic foraminifer packstone and grainstone } \\
\text { Depositional texture: Smaller and larger benthic foraminifer grain-dominated } \\
\text { packstone and grainstone } \\
\text { Color: Very pale orange } 10 \text { YR } 8 / 2 \\
\text { Sedimentary structures: Medium bedded } \\
\text { Trace fossils: Bioturbated } \\
\text { Ichnofabrics: Ichnofabric index } 5 \\
\text { Carbonate grains: Mainly smaller benthic foraminifera, larger benthic foraminifera } \\
\text { (including Fallotella), peloids } \\
\text { Porosity and permeability: } 1-18 \text { percent interparticle and intraparticle porosity, } 1-5 \\
\text { percent moldic porosity; } 2-23 \text { percent total porosity and relatively moderate } \\
\text { permeability } \\
\text { Depositional environment: High-energy inner platform, shallow subtidal }\end{array}$ \\
\hline $\begin{array}{l}\text { obi } \\
\text { depth: } \\
\text { 1,136.3- } \\
\text { 1,136.9 ft } \\
\text { bls } \\
\text { Driller's } \\
\text { depth: } \\
\text { 1,135.4- } \\
\text { 1,136.0 ft } \\
\text { bls }\end{array}$ & $\begin{array}{l}\text { Lithofacies: Benthic foraminifer wackestone and packstone } \\
\text { Depositional texture: Smaller and larger benthic foraminifer mud-dominated packstone } \\
\text { Color: Very pale orange } 10 \text { YR } 8 / 2 \\
\text { Sedimentary structures: Medium bedded } \\
\text { Trace fossils: Bioturbated } \\
\text { Ichnofabrics: Ichnofabric index } 4-5 \\
\text { Carbonate grains: Mainly smaller benthic foraminifera, larger benthic foraminifera } \\
\text { (including Fallotella, uncommon larger miliolids), peloids } \\
\text { Porosity and permeability: } 1-2 \text { percent intraparticle porosity, } 1-5 \text { percent moldic } \\
\text { porosity; } 2-7 \text { percent total porosity and low permeability } \\
\text { Depositional environment: Low-energy inner platform, shallow subtidal }\end{array}$ \\
\hline
\end{tabular}




\begin{tabular}{|c|c|}
\hline $\begin{array}{l}\text { obi } \\
\text { depth: } \\
\text { 1,136.9- } \\
\text { 1,138.2 ft } \\
\text { bls } \\
\text { Driller's } \\
\text { depth: } \\
\text { 1,136.0- } \\
\text { 1,137.3 ft } \\
\text { bls }\end{array}$ & 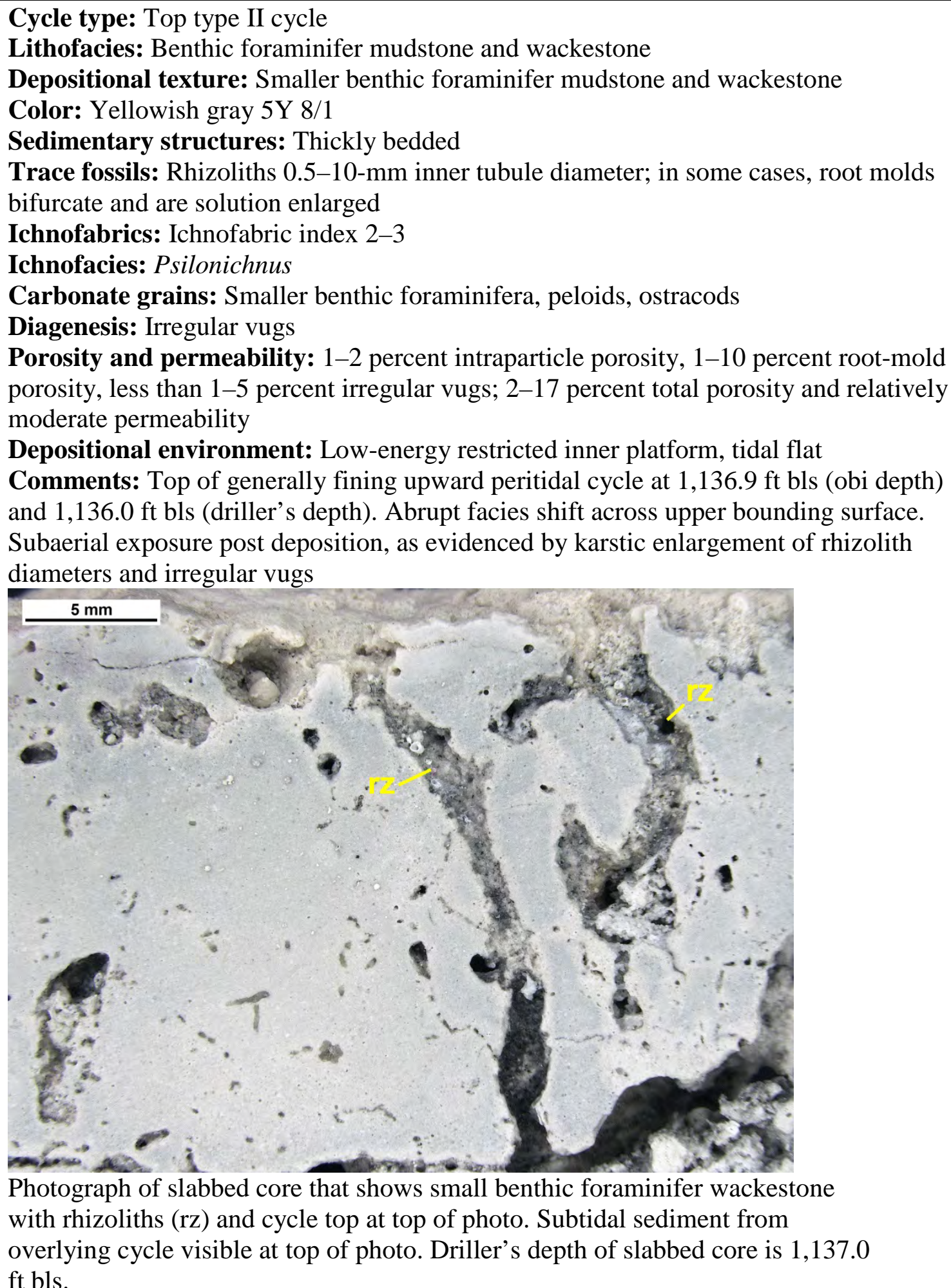 \\
\hline $\begin{array}{l}\text { obi } \\
\text { depth: } \\
1,138.2- \\
1,141.7 \mathrm{ft}\end{array}$ & $\begin{array}{l}\text { Lithofacies: Benthic foraminifer wackestone and packstone } \\
\text { Depositional texture: Smaller and larger benthic foraminifer wackestone and mud- } \\
\text { dominated packstone } \\
\text { Color: Yellowish gray } 5 Y \text { 8/1 }\end{array}$ \\
\hline
\end{tabular}




\begin{tabular}{|c|c|}
\hline $\begin{array}{l}\text { bls } \\
\text { Driller's } \\
\text { depth: } \\
\text { 1,137.3- } \\
\text { 1,140.6 ft } \\
\text { bls }\end{array}$ & $\begin{array}{l}\text { Sedimentary structures: Very thickly bedded } \\
\text { Trace fossils: Bioturbated } \\
\text { Ichnofabrics: Ichnofabric index } 5 \\
\text { Carbonate grains: Smaller benthic foraminifera (including rotaliids, smaller miliolids, } \\
\text { very uncommon Reussella?), larger benthic foraminifera (including Fallotella floridana, } \\
\text { larger miliolids), peloids. Foraminifera observed in thin section G2984-1139.18 include } \\
\text { smaller benthic foraminifera, Fallotella floridana, Pseudochrysalidina?, total of } 14 \\
\text { conical larger benthic foraminifera } \\
\text { Porosity and permeability: 1-5 percent intraparticle porosity, 1-3 percent moldic } \\
\text { porosity, 1-3 percent irregular vugs; 3-11 percent total porosity and relatively low } \\
\text { permeability } \\
\text { Depositional environment: Low-energy restricted inner platform, shallow subtidal } \\
\text { Thin section: G2984-1139.18 } \\
\text { 2 mm }\end{array}$ \\
\hline $\begin{array}{l}\text { obi } \\
\text { depth: } \\
\text { 1,141.7- } \\
\text { 1,145.1 ft } \\
\text { bls } \\
\text { Driller's } \\
\text { depth: } \\
\text { 1,140.6- } \\
\text { 1,144.0 ft } \\
\text { bls }\end{array}$ & $\begin{array}{l}\text { Cycle type: Top type II cycle } \\
\text { Lithofacies: Benthic foraminifer wackestone and packstone } \\
\text { Depositional texture: Smaller benthic foraminifer wackestone and mud-dominated } \\
\text { packstone } \\
\text { Color: Yellowish gray } 5 Y \text { Y } 8 \text { 1 } \\
\text { Sedimentary structures: Thickly bedded, bird's-eye voids, curved- and skew-plane } \\
\text { cracking produced autochthonous brecciation } \\
\text { Trace fossils: Rhizoliths } 0.5-2-m m \text { inner tubule diameter; in some cases, root molds } \\
\text { bifurcate, and in some cases, the inner diameter of the rhizoliths have a semiconcentric } \\
\text { microspar and inner concentric micritization and microbladed calcite cement } \\
\text { Ichnofabrics: Ichnofabric index } 2-3\end{array}$ \\
\hline
\end{tabular}




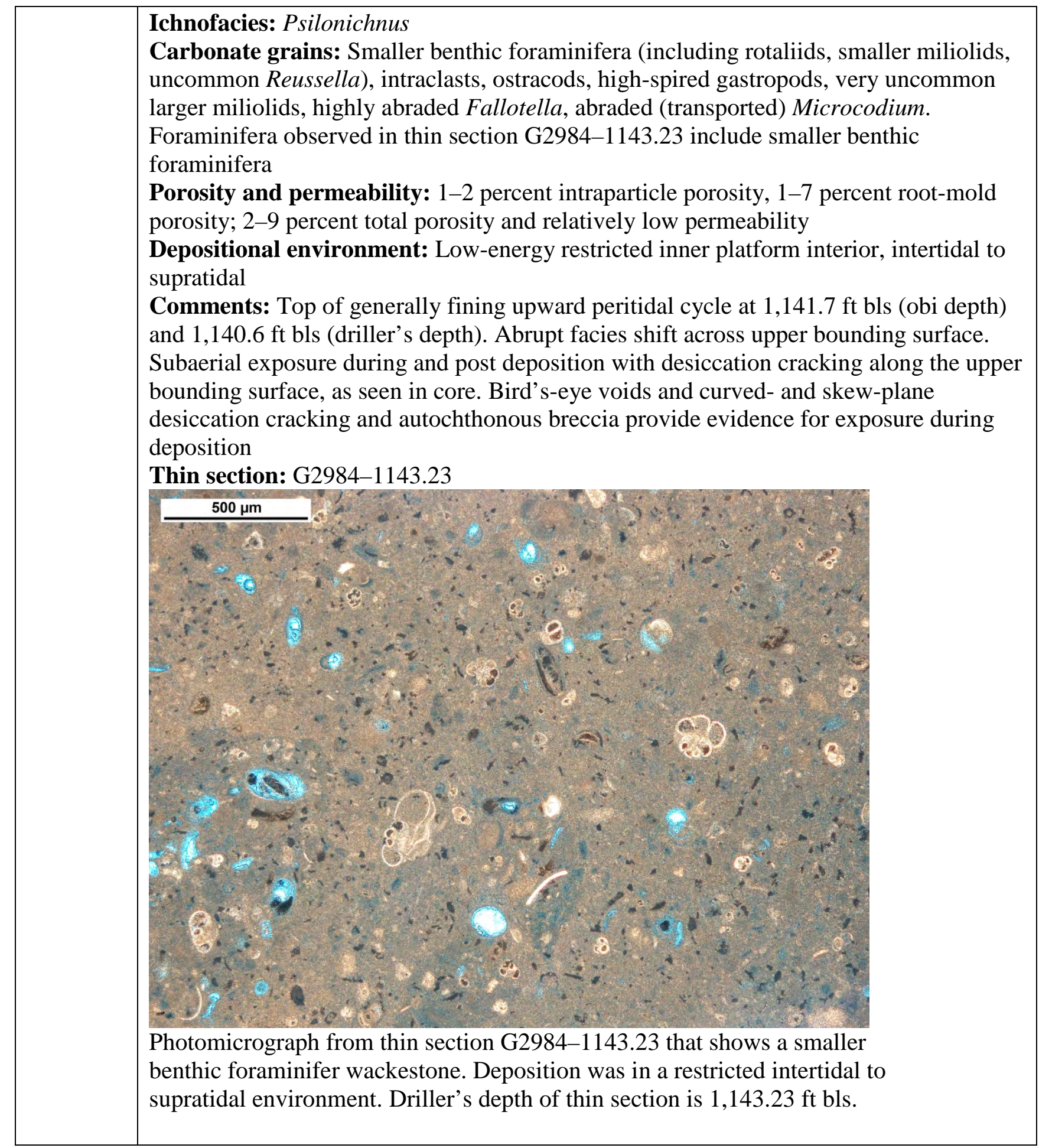




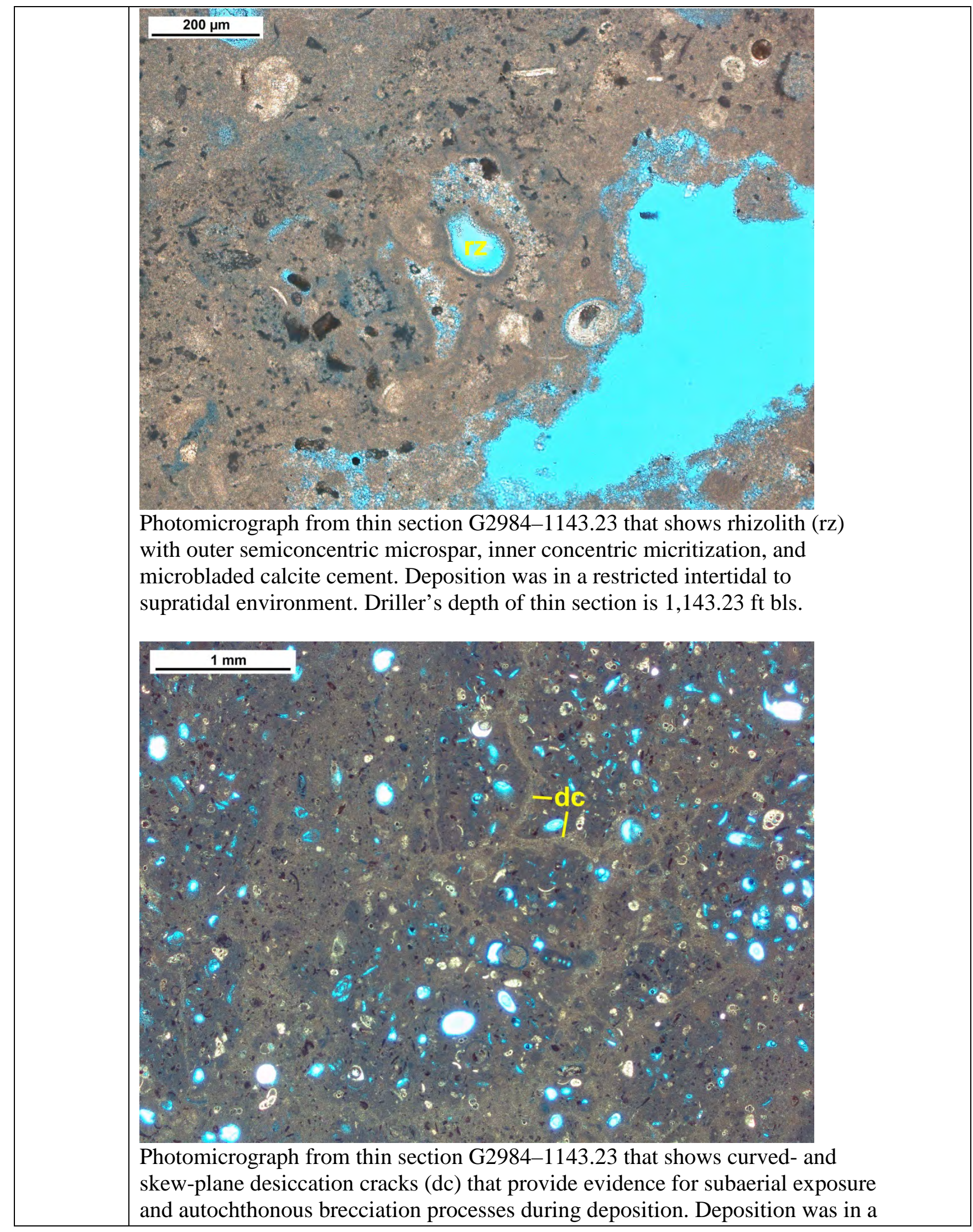




\begin{tabular}{|c|c|}
\hline & $\begin{array}{l}\text { restricted intertidal to supratidal environment. Driller's depth of thin section is } \\
\text { Phot,143.23 ft bls. } \\
\text { foraminifer wackestone and mud-dominated packstone with rhizoliths and } \\
\text { bird's-eye voids. Driller's depth of slabbed core is } 1,142.50 \mathrm{ft} \text { bls. }\end{array}$ \\
\hline $\begin{array}{l}\text { obi } \\
\text { depth: } \\
1,145.1- \\
1,148.9 \mathrm{ft} \\
\text { bls } \\
\text { Driller's } \\
\text { depth: } \\
1,144.0- \\
1,147.5 \mathrm{ft} \\
\text { bls }\end{array}$ & $\begin{array}{l}\text { Lithofacies: Benthic foraminifer packstone and grainstone } \\
\text { Depositional texture: Smaller and larger benthic foraminifer mud- and grain-dominated } \\
\text { packstone } \\
\text { Color: Yellowish gray } 5 Y \text { Y } 1 \\
\text { Sedimentary structures: Very thickly bedded } \\
\text { Trace fossils: Bioturbated } \\
\text { Ichnofabrics: Ichnofabric index } 5 \\
\text { Carbonate grains: Mainly smaller benthic foraminifera, peloids, larger benthic } \\
\text { foraminifera (including Fallotella), ostracods } \\
\text { Porosity and permeability: } 1-12 \text { percent interparticle and intraparticle porosity, } 1-8 \\
\text { percent moldic porosity; } 2-20 \text { percent total porosity and relatively low permeability } \\
\text { Depositional environment: High-energy inner platform, shallow subtidal }\end{array}$ \\
\hline $\begin{array}{l}\text { obi } \\
\text { depth: } \\
1,148.9- \\
1,150.0 \mathrm{ft} \\
\text { bls } \\
\text { Driller's } \\
\text { depth: }\end{array}$ & $\begin{array}{l}\text { Cycle type: Top type II cycle } \\
\text { Lithofacies: Benthic foraminifer wackestone and packstone } \\
\text { Depositional texture: Smaller benthic foraminifer wackestone and mud-dominated } \\
\text { packstone } \\
\text { Color: Yellowish gray } 5 \text { Y } 8 / 1 \\
\text { Sedimentary structures: Thickly bedded, desiccation cracks } \\
\text { Trace fossils: Rhizoliths } 0.5-2-m m \text { inner tubule diameter; in some cases, root molds } \\
\text { bifurcate }\end{array}$ \\
\hline
\end{tabular}




\begin{tabular}{|c|c|}
\hline $\begin{array}{l}1,147.5- \\
1,148.6 \mathrm{ft} \\
\text { bls }\end{array}$ & $\begin{array}{l}\text { Ichnofabrics: Ichnofabric index } 2-3 \\
\text { Ichnofacies: Psilonichnus } \\
\text { Carbonate grains: Smaller benthic foraminifera, peloids, ostracods, gastropods (high } \\
\text { spired), rip-up intraclasts } \\
\text { Porosity and permeability: } 1-2 \text { percent intraparticle porosity, } 1-7 \text { percent root-mold } \\
\text { porosity; } 2-9 \text { percent total porosity and relatively low permeability } \\
\text { Depositional environment: Low-energy, restricted inner platform, tidal flat } \\
\text { Comments: Top of generally fining upward peritidal cycle at 1,148.9 ft bls (obi depth) } \\
\text { and 1,147.5 ft bls (driller's depth). Abrupt facies shift across upper bounding surface }\end{array}$ \\
\hline $\begin{array}{l}\text { obi } \\
\text { depth: } \\
1,150.0- \\
\text { 1,151.3 ft } \\
\text { bls } \\
\text { Driller's } \\
\text { depth: } \\
\text { 1,148.6- } \\
\text { 1,149.2 ft } \\
\text { bls }\end{array}$ & $\begin{array}{l}\text { Lithofacies: Benthic foraminifer wackestone and packstone } \\
\text { Depositional texture: Smaller benthic foraminifer wackestone and mud-dominated } \\
\text { packstone } \\
\text { Color: Yellowish gray 5Y 8/1 } \\
\text { Sedimentary structures: Thickly bedded with paper-thin wispy laminations } \\
\text { Trace fossils: Bioturbated, Planolites?, small Thalassinoides? } \\
\text { Ichnofabrics: Ichnofabric index } 5 \\
\text { Ichnofacies: Proximal Planolites-Thalassinoides-dominated Cruziana } \\
\text { Carbonate grains: Mainly smaller benthic foraminifera, peloids, ostracods, uncommon } \\
\text { larger miliolids, Fallotella } \\
\text { Porosity and permeability: 1-2 percent intraparticle porosity, 1-10 percent moldic } \\
\text { porosity; } 2-12 \text { percent total porosity and relatively low permeability } \\
\text { Depositional environment: Low-energy restricted lagoon inner platform, subtidal }\end{array}$ \\
\hline & $\begin{array}{l}\text { Photograph of slabbed core that shows a smaller foraminifer wackestone and } \\
\text { packstone with wispy laminations. Bioturbation represents a proximal } \\
\text { Planolites-Thalassinoides-dominated Cruziana ichnofacies and low-energy } \\
\text { restricted lagoon. Driller's depth of section is } 1,148.9 \mathrm{ft} \text { bls. }\end{array}$ \\
\hline obi & Top type II \\
\hline
\end{tabular}




\begin{tabular}{|c|c|}
\hline $\begin{array}{l}\text { depth: } \\
\text { 1,151.3- } \\
\text { 1,152.4 ft } \\
\text { bls } \\
\text { Driller's } \\
\text { depth: } \\
\text { 1,149.2- } \\
\text { 1,150.3 ft } \\
\text { bls }\end{array}$ & $\begin{array}{l}\text { Lithofacies: Benthic foraminifer wackestone and packstone } \\
\text { Depositional texture: Smaller benthic foraminifer wackestone and mud-dominated } \\
\text { packstone } \\
\text { Color: Yellowish gray } 5 Y \text { Y } 1 \\
\text { Sedimentary structures: Medium bedded } \\
\text { Trace fossils: Rhizoliths } 0.5-1 \text {-mm inner tubule diameter; in some cases, root molds } \\
\text { bifurcate } \\
\text { Ichnofabrics: Ichnofabric index } 2 \\
\text { Ichnofacies: Psilonichnus } \\
\text { Carbonate grains: Smaller benthic foraminifera, ostracods, uncommon larger miliolids, } \\
\text { peloids } \\
\text { Porosity and permeability: } 1-2 \text { percent intraparticle porosity, } 1-5 \text { percent root-mold } \\
\text { porosity; } 2-7 \text { percent total porosity and relatively low permeability } \\
\text { Depositional environment: Low-energy restricted inner platform, tidal flat } \\
\text { Comments: Top of generally fining upward peritidal cycle at 1,151.3 ft bls (obi depth) } \\
\text { and } 1,149.2 \text { ft bls (driller's depth). Abrupt facies shift across upper bounding surface. } \\
\text { Upper cycle cap has minor desiccation cracking and overlying intraclasts of this interval } \\
\text { at base of superjacent cycle. }\end{array}$ \\
\hline $\begin{array}{l}\text { obi } \\
\text { depth: } \\
1,152.4- \\
1,155.0 \mathrm{ft} \\
\text { bls } \\
\text { Driller's } \\
\text { depth: } \\
1,150.3- \\
1,153.0 \mathrm{ft} \\
\text { bls }\end{array}$ & $\begin{array}{l}\text { Lithofacies: Benthic foraminifer packstone and grainstone } \\
\text { Depositional texture: Smaller foraminifer mud- and grain-dominated packstone } \\
\text { Color: Very pale orange } 10 \text { YR } 8 / 2 \\
\text { Sedimentary structures: Thickly bedded } \\
\text { Trace fossils: Bioturbated } \\
\text { Ichnofabrics: Ichnofabric index } 5 \\
\text { Carbonate grains: Mainly smaller benthic foraminifera, peloids, minor ostracods, } \\
\text { larger benthic foraminifera (including Fallotella, larger miliolids) } \\
\text { Porosity and permeability: } 1-10 \text { percent interparticle and intraparticle porosity, } 1-5 \\
\text { percent moldic porosity; } 2-15 \text { percent total porosity and low permeability } \\
\text { Depositional environment: High-energy inner platform, shallow subtidal }\end{array}$ \\
\hline $\begin{array}{l}\text { obi } \\
\text { depth: } \\
\text { 1,155.0- } \\
\text { 1,155.6 ft } \\
\text { bls } \\
\text { Driller's } \\
\text { depth: } \\
\text { 1,153.0- } \\
\text { 1,153.6 ft } \\
\text { bls }\end{array}$ & $\begin{array}{l}\text { Cycle type: Top type II cycle } \\
\text { Lithofacies: Benthic foraminifer mudstone and wackestone } \\
\text { Depositional texture: Smaller benthic foraminifer wackestone } \\
\text { Color: Very pale orange 10YR 8/2 } \\
\text { Sedimentary structures: Medium bedded, fenestral fabrics } \\
\text { Trace fossils: Rhizoliths } 0.5-3 \mathrm{~mm} \text { inner tubule diameter; in some cases, root molds } \\
\text { bifurcate } \\
\text { Ichnofabrics: Ichnofabric index } 2 \\
\text { Ichnofacies: Psilonichnus } \\
\text { Carbonate grains: Smaller benthic foraminifera (including rotaliids), intraclasts, } \\
\text { ostracods, uncommon larger miliolids, peloids, very uncommon discoidal larger benthic } \\
\text { foraminifera, dasycladacean algae. Foraminifera observed in thin section G2984- } \\
\text { 1153.08 include smaller benthic foraminifera, larger valvulinids } \\
\text { Diagenesis: Curved-plane desiccation cracks } \\
\text { Porosity and permeability: } 1-2 \text { percent intraparticle porosity; } 1-2 \text { percent total } \\
\text { porosity and relatively low permeability } \\
\text { Depositional environment: Low-energy restricted inner platform, intertidal to }\end{array}$ \\
\hline
\end{tabular}




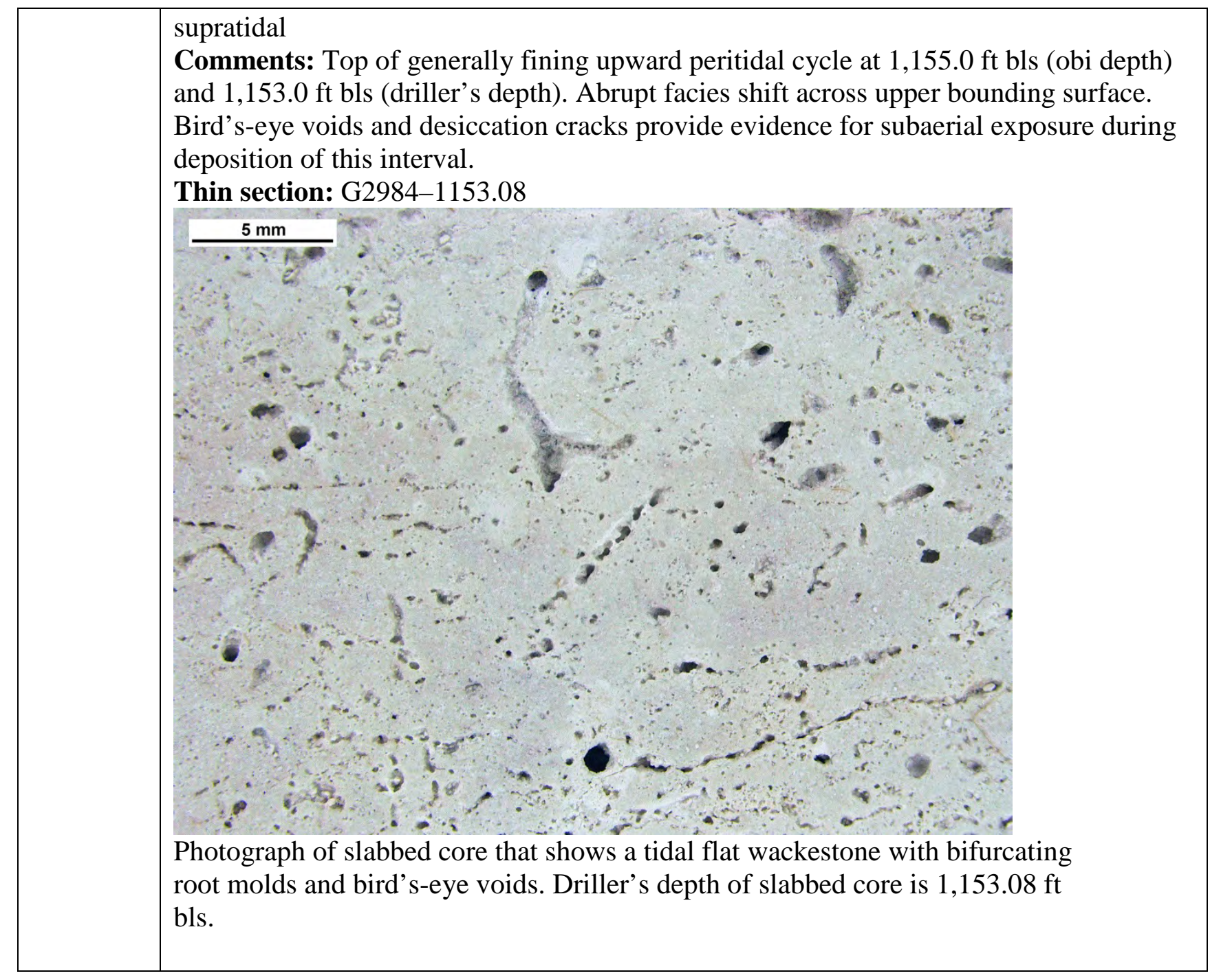




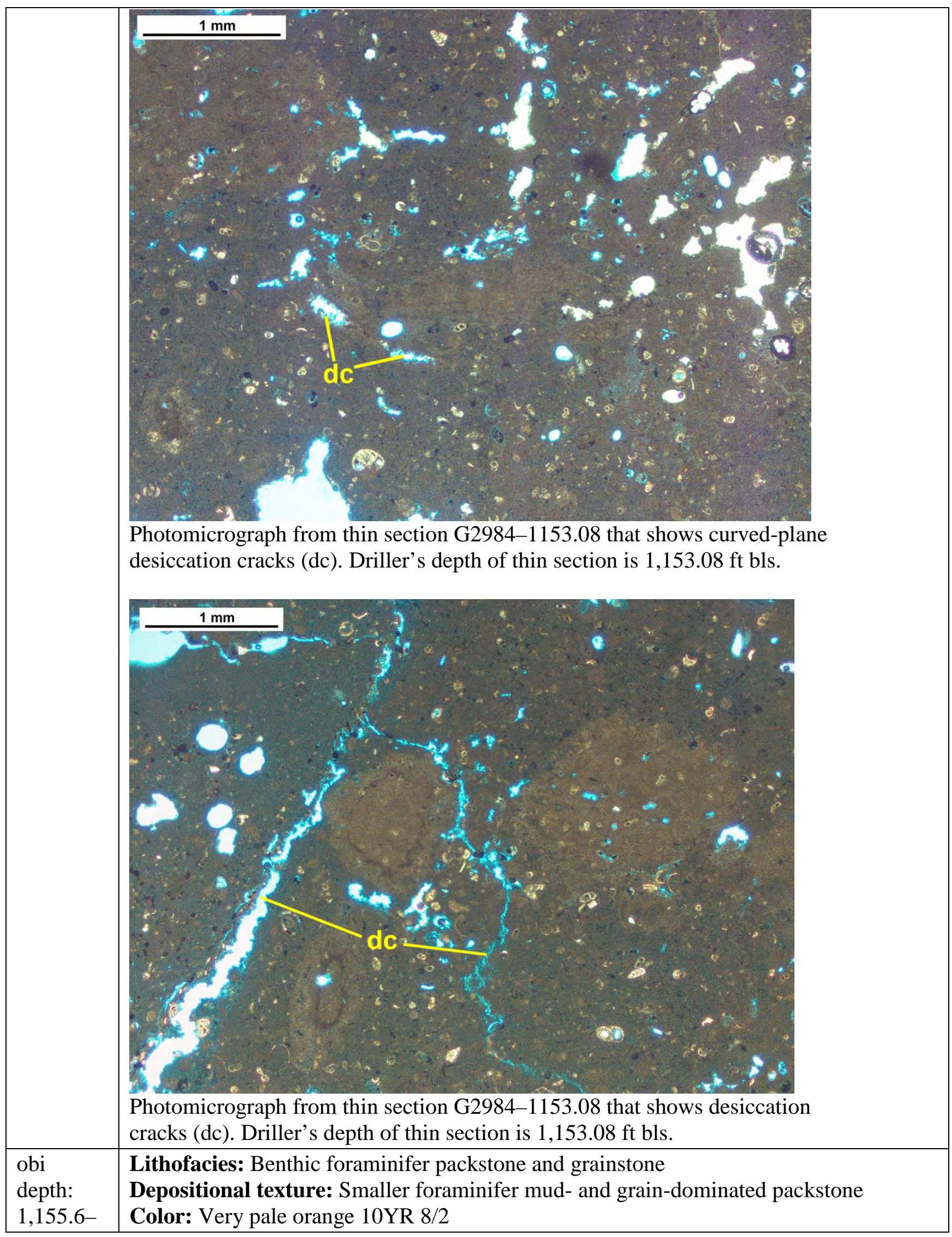




\begin{tabular}{|c|c|}
\hline $\begin{array}{l}1,156.8 \mathrm{ft} \\
\text { bls } \\
\text { Driller's } \\
\text { depth: } \\
1,153.6- \\
1,154.8 \mathrm{ft} \\
\text { bls }\end{array}$ & $\begin{array}{l}\text { Sedimentary structures: Thickly bedded } \\
\text { Trace fossils: Bioturbated } \\
\text { Ichnofabrics: Ichnofabric index } 5 \\
\text { Carbonate grains: Mainly smaller benthic foraminifera, peloids, minor ostracods, } \\
\text { uncommon larger benthic foraminifera (including Fallotella) } \\
\text { Porosity and permeability: } 1-10 \text { percent interparticle and intraparticle porosity, 1-5 } \\
\text { percent moldic porosity; } 2-15 \text { percent total porosity and low permeability } \\
\text { Depositional environment: High-energy inner platform, shallow subtidal }\end{array}$ \\
\hline $\begin{array}{l}\text { obi } \\
\text { depth: } \\
\text { 1,156.8- } \\
\text { 1,158.1 ft } \\
\text { bls } \\
\text { Driller's } \\
\text { depth: } \\
\text { 1,154.8- } \\
\text { 1,156.1 ft } \\
\text { bls }\end{array}$ & $\begin{array}{l}\text { Lithofacies: Benthic foraminifer packstone and grainstone } \\
\text { Depositional texture: Smaller and larger benthic foraminifer mud- and grain-dominated } \\
\text { packstone } \\
\text { Color: Very pale orange 10YR 8/2 } \\
\text { Sedimentary structures: Thickly bedded } \\
\text { Trace fossils: Bioturbated } \\
\text { Ichnofabrics: Ichnofabric index } 5 \\
\text { Carbonate grains: Mainly smaller benthic foraminifera, peloids, and larger benthic } \\
\text { foraminifera (including Fallotella), minor ostracods } \\
\text { Porosity and permeability: } 5-15 \text { percent interparticle and intraparticle porosity, 1-5 } \\
\text { percent moldic porosity; 6-20 percent total porosity and low permeability } \\
\text { Depositional environment: High-energy inner platform, shallow subtidal }\end{array}$ \\
\hline $\begin{array}{l}\text { obi } \\
\text { depth: } \\
\text { 1,158.1- } \\
\text { 1,158.4 ft } \\
\text { bls } \\
\text { Driller's } \\
\text { depth: } \\
\text { 1,156.1- } \\
\text { 1,156.4 ft } \\
\text { bls }\end{array}$ & $\begin{array}{l}\text { Lithofacies: Benthic foraminifer mudstone and wackestone } \\
\text { Depositional texture: Smaller benthic foraminifer wackestone } \\
\text { Color: Very pale orange } 10 \mathrm{YR} 8 / 2 \text { and pale yellowish brown } 10 \mathrm{YR} 6 / 2 \\
\text { Sedimentary structures: Very thinly bedded with paper-thin laminations } \\
\text { Ichnofabrics: Ichnofabric index } 2-3 \\
\text { Carbonate grains: Smaller benthic foraminifera and peloids, uncommon larger benthic } \\
\text { foraminifera } \\
\text { Accessory grains: } 1 \text { percent carbonaceous fragments of plants-paper-thin } \\
\text { discontinuous accumulations parallel to laminations } \\
\text { Porosity and permeability: } 1-2 \text { percent intraparticle porosity; } 1-2 \text { percent total } \\
\text { porosity and relatively low permeability } \\
\text { Depositional environment: Low-energy restricted inner platform, shallow subtidal } \\
\text { Comments: Transgressive base of cycle }\end{array}$ \\
\hline
\end{tabular}




\begin{tabular}{|c|c|}
\hline & $\begin{array}{l}\text { Photograph of slabbed core that shows a small benthic foraminifer wackestone } \\
\text { that is the transgressive base of a high-frequency cycle. Driller's depth of } \\
\text { slabbed core is } 1,159.8 \mathrm{ft} \text { bls. }\end{array}$ \\
\hline $\begin{array}{l}\text { obi } \\
\text { depth: } \\
\text { 1,158.4- } \\
\text { 1,160.9 ft } \\
\text { bls } \\
\text { Driller's } \\
\text { depth: } \\
\text { 1,156.4- } \\
\text { 1,158.9 ft } \\
\text { bls }\end{array}$ & $\begin{array}{l}\text { Cycle type: Top type II cycle } \\
\text { Lithofacies: Benthic foraminifer wackestone and packstone } \\
\text { Depositional texture: Smaller benthic foraminifer wackestone and mud-dominated } \\
\text { packstone } \\
\text { Color: Very pale orange 10YR 8/2 (wet) } \\
\text { Sedimentary structures: Thickly bedded } \\
\text { Trace fossils: Rhizoliths } 0.5-3-\text {-mm inner tubule diameter } \\
\text { Ichnofabrics: Ichnofabric index } 2 \\
\text { Ichnofacies: Psilonichnus } \\
\text { Carbonate grains: Mainly smaller benthic foraminifera, peloids, minor ostracods, high- } \\
\text { spired gastropods, uncommon larger miliolids } \\
\text { Porosity and permeability: } 1 \text { percent intraparticle porosity, 1-5 percent fossil molds, } \\
\text { 1-5 percent root molds; 3-11 percent total porosity and relatively low permeability } \\
\text { Depositional environment: Low-energy restricted inner platform, tidal flat } \\
\text { Comments: Top of generally fining upward peritidal cycle at 1,158.4 ft bls (obi depth) } \\
\text { and 1,156.4 ft bls (driller's depth). Probable subaerial exposure at upper bounding } \\
\text { surface where there is an abrupt shift in lithofacies }\end{array}$ \\
\hline $\begin{array}{l}\text { obi } \\
\text { depth: } \\
1,160.9- \\
1,163.1 \mathrm{ft} \\
\text { bls } \\
\text { Driller's }\end{array}$ & $\begin{array}{l}\text { Lithofacies: Benthic foraminifer packstone and grainstone } \\
\text { Depositional texture: Smaller and larger benthic foraminifer mud- and grain-dominated } \\
\text { packstone and grainstone } \\
\text { Color: Very pale orange } 10 \text { YR } 8 / 2 \\
\text { Sedimentary structures: Thickly bedded } \\
\text { Trace fossils: Bioturbated } \\
\text { Ichnofabrics: Ichnofabric index } 5\end{array}$ \\
\hline
\end{tabular}




\begin{tabular}{|c|c|}
\hline $\begin{array}{l}\text { depth: } \\
\text { 1,158.9- } \\
\text { 1,162.1 ft } \\
\text { bls }\end{array}$ & $\begin{array}{l}\text { Carbonate grains: Mainly smaller benthic foraminifera, peloids, and larger benthic } \\
\text { foraminifera (including Fallotella), gastropods (including high-spired gastropods), } \\
\text { minor ostracods } \\
\text { Porosity and permeability: 5-20 percent interparticle and intraparticle porosity, 1-5 } \\
\text { percent moldic porosity; 6-25 percent total porosity and low to moderate permeability } \\
\text { Depositional environment: High-energy inner platform, shallow subtidal }\end{array}$ \\
\hline $\begin{array}{l}\text { obi } \\
\text { depth: } \\
\text { 1,163.1- } \\
\text { 1,167.9 ft } \\
\text { bls } \\
\text { Driller's } \\
\text { depth: } \\
\text { 1,162.1- } \\
\text { 1,166.7 ft } \\
\text { bls }\end{array}$ & $\begin{array}{l}\text { Cycle type: Top type II cycle } \\
\text { Lithofacies: Benthic foraminifer wackestone and packstone } \\
\text { Depositional texture: Smaller benthic foraminifer wackestone and mud-dominated } \\
\text { packstone } \\
\text { Color: Very light gray N8 } \\
\text { Sedimentary structures: Thickly bedded, fenestral fabric in uppermost } 2 \text { in., curved } \\
\text { plane desiccation cracks } \\
\text { Trace fossils: Rhizoliths } 0.5-5-\text { mm inner tubule diameter (in a few cases with } \\
\text { microspar lining inner wall); in some cases, solution enlarged inner diameter } \\
\text { Ichnofabrics: Ichnofabric index } 2 \\
\text { Ichnofacies: Psilonichnus for the uppermost } 1 \text { ft } \\
\text { Carbonate grains: Mainly smaller benthic foraminifera (including rotaliids, } \\
\text { Reussella?), peloids, larger benthic foraminifera (including larger miliolids, Fallotella } \\
\text { floridana), ostracods, uncommon gastropods, small bivalves, Microcodium. } \\
\text { Foraminifera observed in thin section G2984-1162.82 include smaller benthic } \\
\text { foraminifera, Fallotella floridana, total of nine conical larger benthic foraminifera } \\
\text { Porosity and permeability: } 1 \text { percent intraparticle porosity, } 1-3 \text { percent fossil molds, } 1 \\
\text { percent local fenestral fabric porosity, } 1-5 \text { percent irregular vugs; } 4-11 \text { percent total } \\
\text { porosity and relatively low permeability } \\
\text { Depositional environment: Low-energy restricted inner platform, intertidal to } \\
\text { supratidal } \\
\text { Comments: Uppermost } 2 \text { in. is definitively intertidal with fenestral fabric and very } \\
\text { restricted fauna (smaller benthic foraminifera wackestone and mud-dominated } \\
\text { packstone, no thin section here to accurately identify particle types). Top of generally } \\
\text { fining upward peritidal cycle at } 1,163.1 \text { ft bls (obi depth) and } 1,162.1 \text { ft bls (driller's } \\
\text { depth). Subaerial exposure at upper bounding surface } \\
\text { Thin section: G2984-1162.82 }\end{array}$ \\
\hline
\end{tabular}




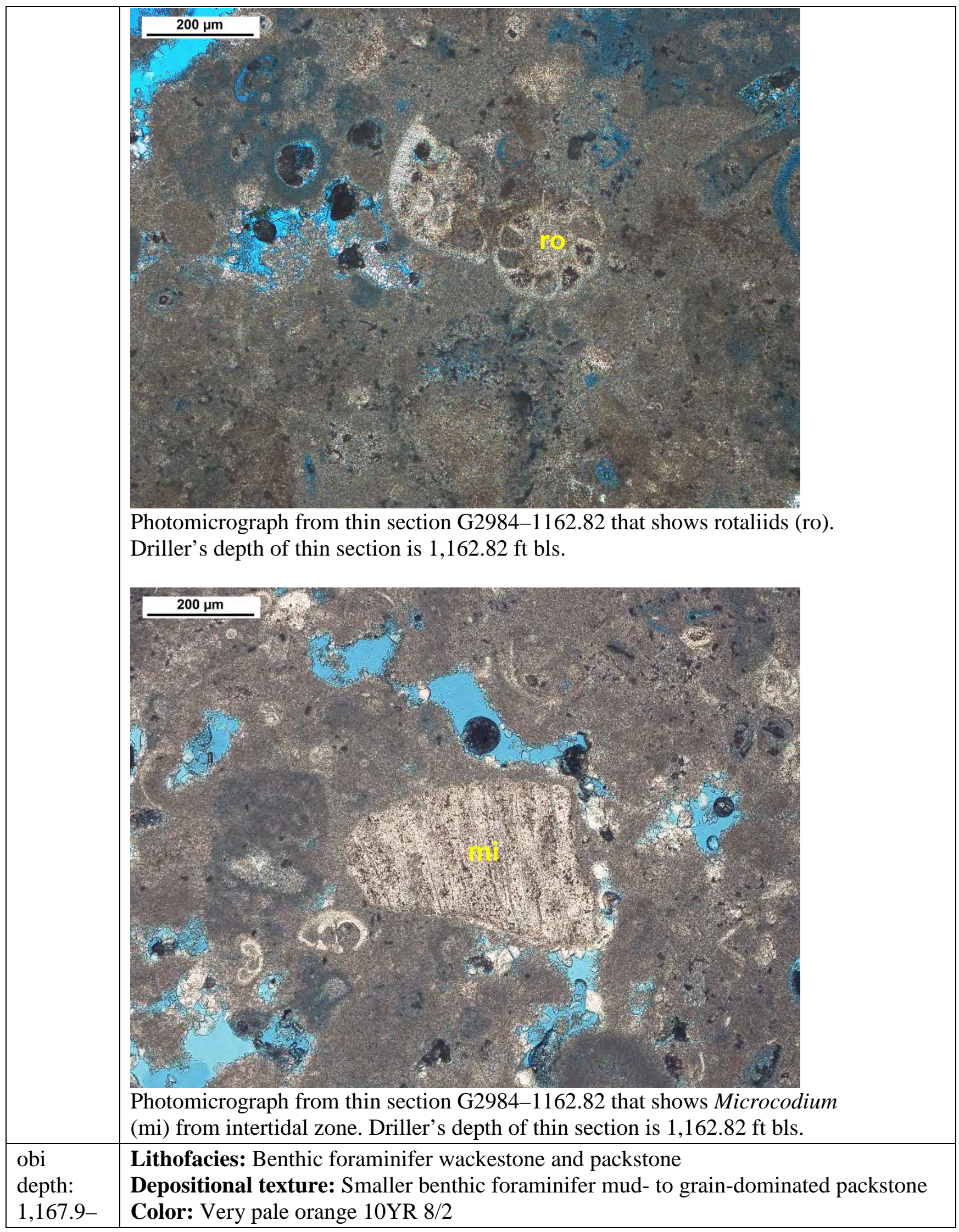




\begin{tabular}{|c|c|}
\hline $\begin{array}{l}\text { 1,170.6 ft } \\
\text { bls } \\
\text { Driller's } \\
\text { depth: } \\
\text { 1,166.7- } \\
1,169.35 \\
\text { ft bls }\end{array}$ & $\begin{array}{l}\text { Sedimentary structures: Thickly bedded } \\
\text { Trace fossils: Burrow mottled } \\
\text { Ichnofabrics: Ichnofabric index } 5 \\
\text { Carbonate grains: Mainly smaller benthic foraminifera (including rotaliids), peloids, } \\
\text { minor larger benthic foraminifera (including Fallotella), intraclasts, high-spired } \\
\text { gastropods, ostracods } \\
\text { Porosity and permeability: } 1-15 \text { percent interparticle and intraparticle porosity; 1-15 } \\
\text { percent total porosity and relatively low permeability } \\
\text { Depositional environment: Low-energy inner platform, shallow subtidal }\end{array}$ \\
\hline $\begin{array}{l}\text { obi } \\
\text { depth: } \\
1,170.6- \\
\text { 1,172.6 ft } \\
\text { bls } \\
\text { Driller's } \\
\text { depth: } \\
\text { 1,169.35- } \\
\text { 1,171.35 } \\
\text { ft bls }\end{array}$ & $\begin{array}{l}\text { Cycle type: Top type II cycle } \\
\text { Lithofacies: Benthic foraminifer wackestone and mud-dominated packstone } \\
\text { Depositional texture: Smaller benthic foraminifer wackestone } \\
\text { Color: Very light gray N8 } \\
\text { Sedimentary structures: Thickly bedded } \\
\text { Trace fossils: Rhizoliths } 0.5-5-\text { mm inner tubule diameter; in some cases, solution } \\
\text { enlarged inner diameter } \\
\text { Ichnofabrics: Ichnofabric index } 2 \\
\text { Ichnofacies: Psilonichnus } \\
\text { Carbonate grains: Peloids and smaller benthic foraminifera, gastropods (high spired), } \\
\text { uncommon bivalves } \\
\text { Porosity and permeability: } 1-3 \text { percent interparticle porosity, intraparticle porosity, } \\
\text { and fossil moldic porosity; } 2-5 \text { percent root mold porosity; 3-8 percent total porosity } \\
\text { and relatively low to moderate permeability } \\
\text { Depositional environment: Low-energy restricted inner platform, tidal flat } \\
\text { Comments: Top of generally fining upward peritidal cycle at 1,170.6 ft bls (obi depth) } \\
\text { and } 1,169.35 \text { ft bls (driller's depth). Subaerial exposure at upper bounding surface }\end{array}$ \\
\hline $\begin{array}{l}\text { obi } \\
\text { depth: } \\
1,172.6- \\
1,174.95 \\
\text { ft bls } \\
\text { Driller's } \\
\text { depth: } \\
\text { 1,171.35- } \\
\text { 1,173.7 ft } \\
\text { bls }\end{array}$ & $\begin{array}{l}\text { Lithofacies: Benthic foraminifer wackestone and packstone } \\
\text { Depositional texture: Smaller benthic foraminifer wackestone and mud-dominated } \\
\text { packstone } \\
\text { Color: Upper part of interval very light gray N8 } \\
\text { Sedimentary structures: Thickly bedded } \\
\text { Trace fossils: Bioturbated } \\
\text { Ichnofabrics: Ichnofabric index } 5 \\
\text { Carbonate grains: Mainly smaller benthic foraminifera, unidentified grains, } \\
\text { gastropods, thin-shelled bivalves, possible Fallotella } \\
\text { Porosity and permeability: } 1-18 \text { percent interparticle and intraparticle porosity, 1-8 } \\
\text { percent fossil molds; } 2-26 \text { percent total porosity and relatively low to moderate } \\
\text { permeability } \\
\text { Depositional environment: Low-energy inner platform, shallow subtidal }\end{array}$ \\
\hline $\begin{array}{l}\text { obi } \\
\text { depth: } \\
1,174.95- \\
1,176.25 \\
\text { ft bls }\end{array}$ & $\begin{array}{l}\text { Cycle type: Top type II cycle } \\
\text { Lithofacies: Benthic foraminifer wackestone and packstone } \\
\text { Depositional texture: Smaller benthic foraminifer wackestone } \\
\text { Color: Very light gray N8 } \\
\text { Sedimentary structures: Part of a very thickly bedded unit } \\
\text { Trace fossils: Rhizoliths } 0.5-5-\mathrm{mm} \text { inner tubule diameter; in some cases, solution }\end{array}$ \\
\hline
\end{tabular}




\begin{tabular}{|c|c|}
\hline $\begin{array}{l}\text { Driller's } \\
\text { depth: } \\
1,173.7- \\
1,175.0 \mathrm{ft} \\
\text { bls }\end{array}$ & $\begin{array}{l}\text { enlarged inner diameter. Horizontal lateral mangrove roots with vertical } \\
\text { pneumatophores and geotropic roots } \\
\text { Ichnofabrics: Ichnofabric index } 2 \\
\text { Ichnofacies: Psilonichnus } \\
\text { Carbonate grains: Peloids and smaller benthic foraminifera, larger benthic foraminifer } \\
\text { (larger miliolids and very uncommon Fallotella), gastropods (high spired) } \\
\text { Porosity and permeability: } 1-3 \text { percent interparticle porosity, intraparticle porosity, } \\
\text { and fossil moldic porosity; } 2-5 \text { percent root mold porosity; } 3-8 \text { percent total porosity } \\
\text { and relatively low to moderate permeability } \\
\text { Depositional environment: Low-energy restricted inner platform interior, tidal flat } \\
\text { Comments: Thin (1-4 mm thick) microbial laminite at } 1,181.4 \text { ft bls (obi depth) and } \\
1,174 \text { ft bls (driller's depth). Top of generally fining upward peritidal cycle at } 1,174.95 \\
\text { ft bls (obi depth) and 1,176.25 ft bls (driller's depth). Subaerial exposure at upper } \\
\text { bounding surface }\end{array}$ \\
\hline $\begin{array}{l}\text { obi } \\
\text { depth: } \\
\text { 1,176.25- } \\
\text { 1,186.4 ft } \\
\text { bls } \\
\text { Driller's } \\
\text { depth: } \\
\text { 1,175.0- } \\
\text { 1,185.0 ft } \\
\text { bls }\end{array}$ & $\begin{array}{l}\text { Lithofacies: Benthic foraminifer wackestone and packstone } \\
\text { Depositional texture: Smaller benthic foraminifer wackestone and packstone } \\
\text { Color: Upper part of interval very light gray N8 and lower part very pale orange 10YR } \\
8 / 2 \\
\text { Sedimentary structures: Very thickly bedded } \\
\text { Trace fossils: Bioturbated } \\
\text { Ichnofabrics: Ichnofabric index } 5 \\
\text { Carbonate grains: Mainly smaller benthic foraminifera (including rotaliids, smaller } \\
\text { miliolids, Reussella?), peloids, minor ostracods, uncommon larger miliolids. } \\
\text { Foraminifera observed in thin section G2984-1184.85 include smaller benthic } \\
\text { foraminifera, planktic foraminifera } \\
\text { Porosity and permeability: } 1-18 \text { percent interparticle and intraparticle porosity, 1-8 } \\
\text { percent fossil molds; } 2-26 \text { percent total porosity and relatively low to moderate } \\
\text { permeability } \\
\text { Depositional environment: Low-energy restricted lagoon? inner platform, shallow } \\
\text { subtidal } \\
\text { Thin section: G2984-1184.85 }\end{array}$ \\
\hline
\end{tabular}




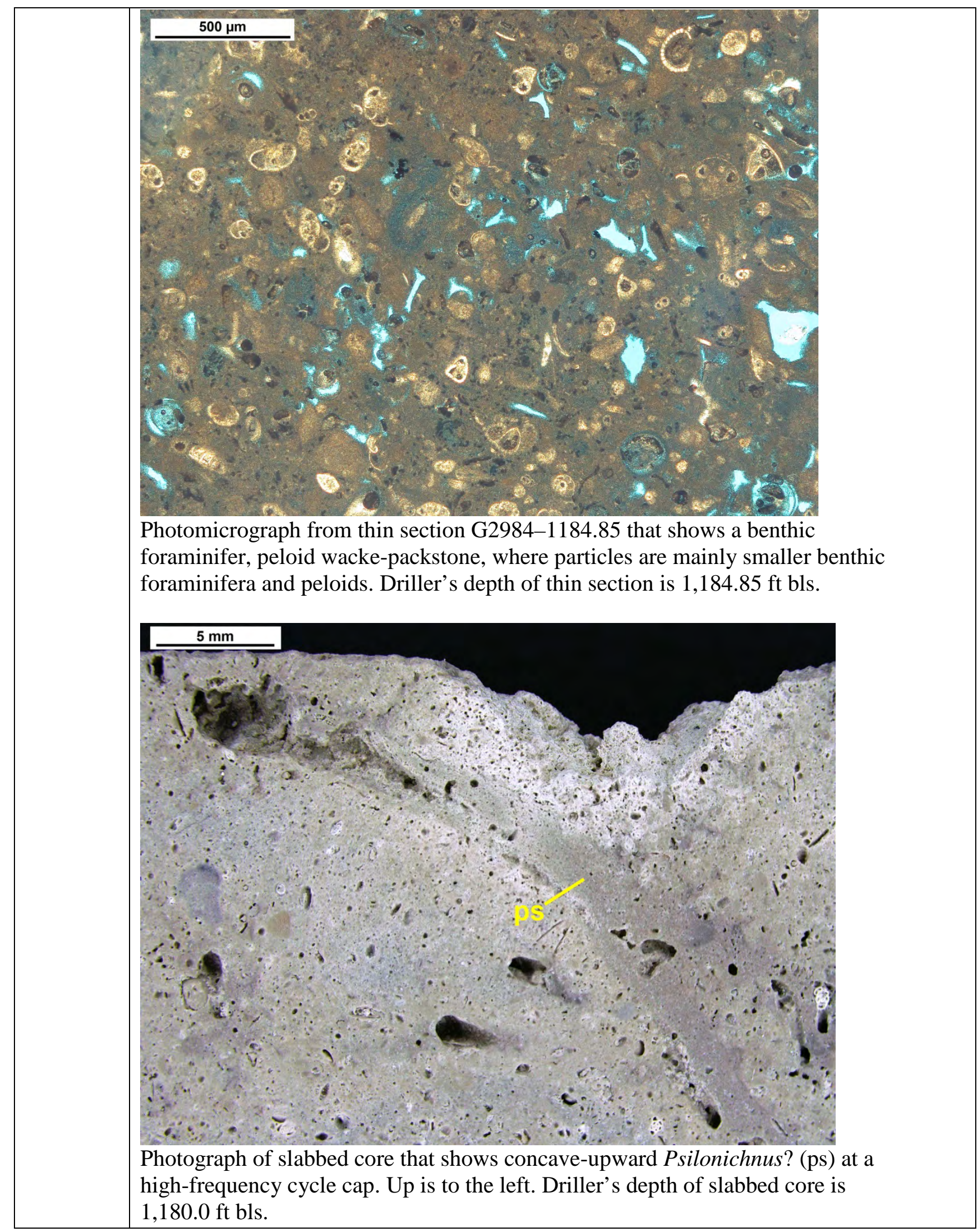




\begin{tabular}{|c|c|}
\hline $\begin{array}{l}\text { obi } \\
\text { depth: } \\
\text { 1,186.4- } \\
\text { 1,189.7 ft } \\
\text { bls } \\
\text { Driller's } \\
\text { depth: } \\
\text { 1,185.0- } \\
\text { 1,188.3 ft } \\
\text { bls }\end{array}$ & $\begin{array}{l}\text { Lithofacies: Benthic foraminifer packstone and grainstone } \\
\text { Depositional texture: Smaller and larger benthic foraminifer mud- and grain-dominated } \\
\text { packstone and grainstone } \\
\text { Color: Very pale orange } 10 \text { YR } 8 / 2 \\
\text { Sedimentary structures: Very thickly bedded } \\
\text { Trace fossils: Bioturbated } \\
\text { Ichnofabrics: Ichnofabric index } 5 \\
\text { Carbonate grains: Mainly smaller benthic foraminifera, peloids, and larger benthic } \\
\text { foraminifera (including Fallotella, larger miliolids), minor ostracods, disarticulated thin } \\
\text { bivalves, fragmented echinoids, very uncommon planktic foraminifera? } \\
\text { Porosity and permeability: } 5-25 \text { percent interparticle and intraparticle porosity, 1-5 } \\
\text { percent moldic porosity; 6-30 percent total porosity and low to moderate permeability } \\
\text { Depositional environment: High-energy inner platform, shallow subtidal }\end{array}$ \\
\hline $\begin{array}{l}\text { obi } \\
\text { depth: } \\
\text { 1,189.7- } \\
\text { 1,190.5 ft } \\
\text { bls } \\
\text { Driller's } \\
\text { depth: } \\
\text { 1,188.3- } \\
\text { 1,189.1 ft } \\
\text { bls }\end{array}$ & $\begin{array}{l}\text { Cycle type: Top type II cycle } \\
\text { Lithofacies: Benthic foraminifer mudstone and wackestone } \\
\text { Depositional texture: Smaller benthic foraminifer wackestone } \\
\text { Color: Very pale orange } 10 \text { YR } 8 / 2 \\
\text { Sedimentary structures: Thinly bedded } \\
\text { Trace fossils: Bioturbated; rhizoliths } 0.5-1.0-\text { mm wide inner tubule diameter } \\
\text { Ichnofabrics: Ichnofabric index } 2-5 \\
\text { Ichnofacies: Psilonichnus } \\
\text { Carbonate grains: Wackestone is mainly smaller benthic foraminifera (including } \\
\text { rotaliids, Reussella), peloids, minor ostracods, echinoid plates, rare larger miliolids, } \\
\text { gastropods, planktic foraminifera. Foraminifera observed in G2984-1188.73 include } \\
\text { smaller benthic foraminifera, Fallotella floridana, total of two conical larger benthic } \\
\text { foraminifera } \\
\text { Porosity and permeability: } 1-12 \text { percent interparticle and intraparticle porosity, 1-2 } \\
\text { percent root molds, } 1-2 \text { percent fossil molds; } 3-16 \text { percent total porosity and relatively } \\
\text { low permeability } \\
\text { Depositional environment: Low-energy restricted inner platform, tidal flat } \\
\text { Comments: Cycle cap at } 1,189.7 \text { ft bls (obi depth) and 1,188.3 ft bls (driller's depth). } \\
\text { Upper bounding surface is an exposure surface } \\
\text { Thin section: G2984-1188.73 }\end{array}$ \\
\hline
\end{tabular}




\begin{tabular}{|c|c|}
\hline & $\begin{array}{l}\text { Photomicrograph from thin section G2984-1188.73 that shows a Reussella (re), } \\
\text { rotaliid (ro), and ostracod (os). Driller's depth of section is } 1,188.73 \mathrm{ft} \text { bls. }\end{array}$ \\
\hline $\begin{array}{l}\text { obi } \\
\text { depth: } \\
\text { 1,190.5- } \\
\text { 1,192.5 ft } \\
\text { bls } \\
\text { Driller's } \\
\text { depth: } \\
\text { 1,189.1- } \\
\text { 1,191 ft } \\
\text { bls }\end{array}$ & $\begin{array}{l}\text { Lithofacies: Benthic foraminifer packstone and grainstone } \\
\text { Depositional texture: Smaller and larger grain-dominated benthic foraminifer mud- and } \\
\text { grain-dominated packstone and grainstone } \\
\text { Color: Very pale orange } 10 \text { YR } 8 / 2 \\
\text { Sedimentary structures: Thickly bedded } \\
\text { Trace fossils: Bioturbated } \\
\text { Ichnofabrics: Ichnofabric index } 5 \\
\text { Carbonate grains: Mainly smaller benthic foraminifera, peloids, intraclasts, and larger } \\
\text { benthic foraminifera (including very uncommon Fallotella floridana), minor ostracods, } \\
\text { disarticulated thin bivalves, fragmented echinoids } \\
\text { Porosity and permeability: } 5-25 \text { percent interparticle and intraparticle porosity, } 1-5 \\
\text { percent moldic porosity; } 6 \text {-30 percent total porosity and low to moderate permeability } \\
\text { Depositional environment: High-energy inner platform interior, shallow subtidal }\end{array}$ \\
\hline $\begin{array}{l}\text { obi } \\
\text { depth: } \\
\text { 1,192.5- } \\
\text { 1,198.0 ft } \\
\text { bls } \\
\text { Driller's } \\
\text { depth: } \\
\text { 1,191- } \\
\text { 1,196.7 ft } \\
\text { bls }\end{array}$ & $\begin{array}{l}\text { Cycle type: Top type II cycle } \\
\text { Lithofacies: Benthic foraminifer wackestone and packstone } \\
\text { Depositional texture: Smaller benthic foraminifer wackestone and mud-dominated } \\
\text { packstone } \\
\text { Color: Very pale orange } 10 \text { YR } 8 / 2 \\
\text { Sedimentary structures: Very thickly bedded } \\
\text { Trace fossils: Bioturbated; rhizoliths in uppermost part and upper-middle part of cycle } \\
\text { with 0.5-1.0-mm wide inner tubule diameter and deeper penetrating vertical, rhizoliths } \\
\text { with 5-mm wide inner tubule diameter } \\
\text { Ichnofabrics: Ichnofabric index } 2-5 \\
\text { Ichnofacies: Psilonichnus in uppermost part of cycle } \\
\text { Carbonate grains: Wackestone is mainly smaller benthic foraminifera (including }\end{array}$ \\
\hline
\end{tabular}




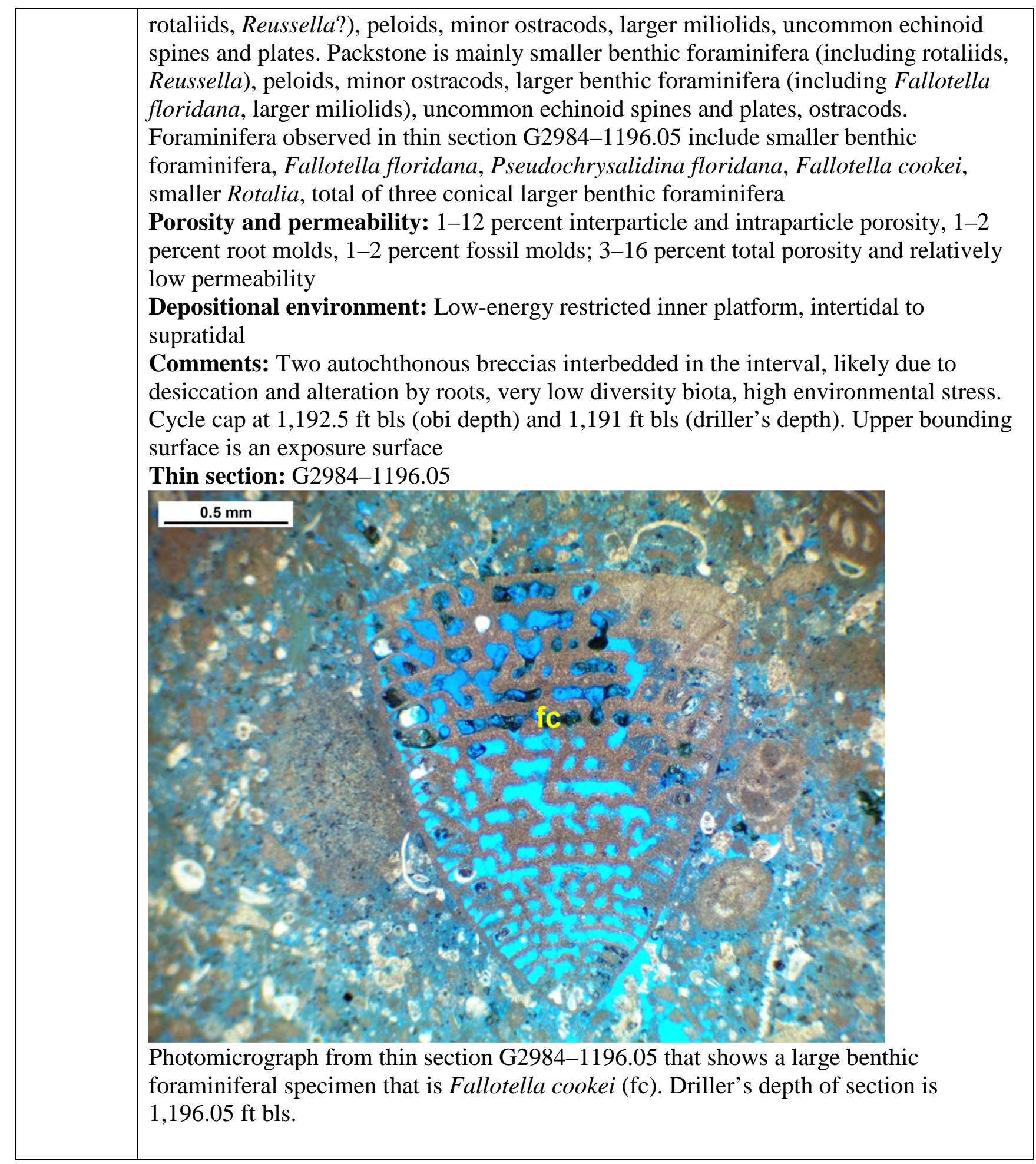




\begin{tabular}{|c|c|}
\hline & $\begin{array}{l}\text { Photomicrograph from thin section G2984-1196.05 that shows a large benthic } \\
\text { foraminiferal specimen that is Pseudochrysalidina floridana (pf). Driller's depth } \\
\text { of section is 1,196.05 ft bls. }\end{array}$ \\
\hline $\begin{array}{l}\text { obi } \\
\text { depth: } \\
1,198.0- \\
1,199.6 \mathrm{ft} \\
\text { bls } \\
\text { Driller's } \\
\text { depth: } \\
1,196.7- \\
1,198.25 \\
\text { ft bls }\end{array}$ & $\begin{array}{l}\text { Lithofacies: Benthic foraminifer packstone and grainstone } \\
\text { Depositional texture: Smaller and larger benthic foraminifer mud- and grain-dominated } \\
\text { packstone and grainstone } \\
\text { Color: Very pale orange } 10 \text { YR } 8 / 2 \\
\text { Sedimentary structures: Thickly bedded } \\
\text { Trace fossils: Bioturbated } \\
\text { Ichnofabrics: Ichnofabric index } 5 \\
\text { Carbonate grains: Mainly peloids, smaller benthic foraminifera (including rotaliids, } \\
\text { biserials, miliolids, Reussella?), intraclasts, and larger benthic foraminifera (including } \\
\text { Fallotella cookei and larger miliolids), minor ostracods, disarticulated thin bivalves } \\
\text { Porosity and permeability: } 5-25 \text { percent interparticle and intraparticle porosity, } 1-5 \\
\text { percent moldic porosity; } 6 \text {-30 percent total porosity and low to moderate permeability } \\
\text { Depositional environment: High-energy inner platform, shallow subtidal }\end{array}$ \\
\hline $\begin{array}{l}\text { obi } \\
\text { depth: } \\
1,199.6- \\
1,205.3 \mathrm{ft} \\
\text { bls } \\
\\
\text { Driller's } \\
\text { depth: } \\
1,198.25- \\
1,204.0 \mathrm{ft}\end{array}$ & $\begin{array}{l}\text { Cycle type: Top type II cycle } \\
\text { Lithofacies: Benthic foraminifer wackestone and packstone } \\
\text { Depositional texture: Smaller benthic foraminifer wackestone and mud-dominated } \\
\text { packstone } \\
\text { Color: Very pale orange } 10 \text { YR } 8 / 2 \\
\text { Sedimentary structures: Very thickly bedded } \\
\text { Trace fossils: Bioturbated; rhizoliths in uppermost part of cycle, } 0.5-1.0 \text {-mm wide inner } \\
\text { tubule diameter, deeper penetrating, vertical, rhizoliths with 5-mm wide inner tubule } \\
\text { diameter based on acoustic borehole wall image and examples in core samples—-some } \\
\text { tubules solution enlarged }\end{array}$ \\
\hline
\end{tabular}




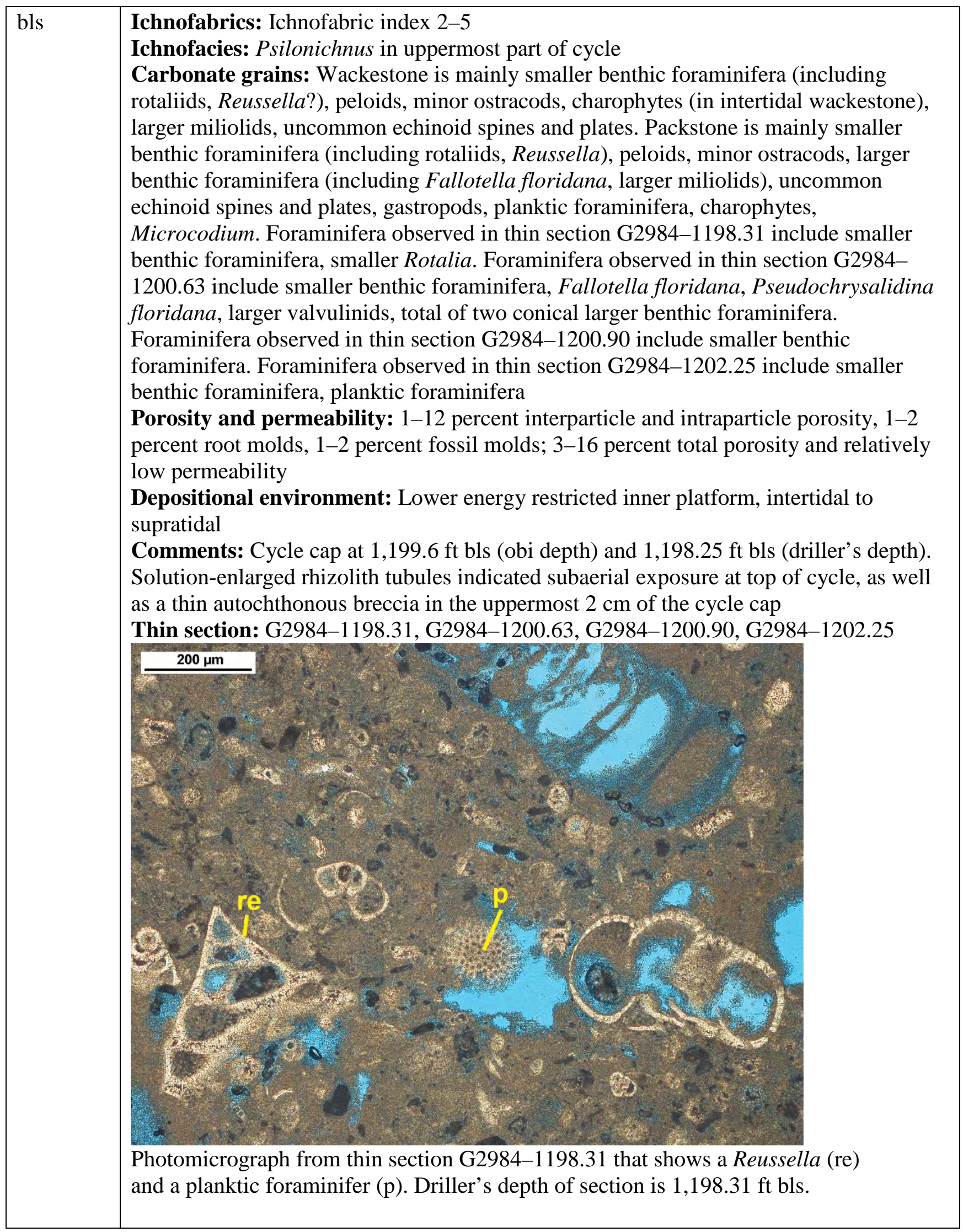




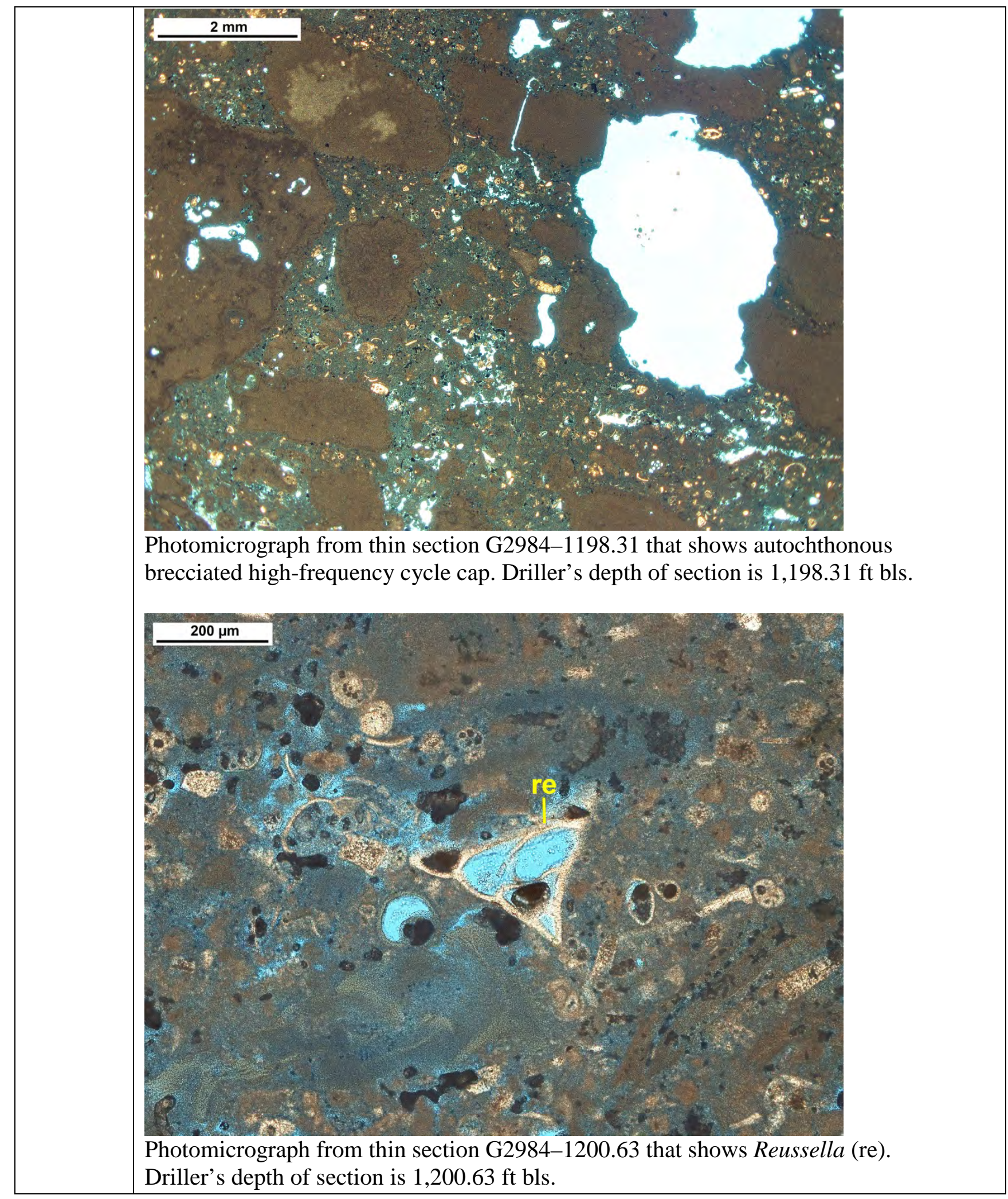




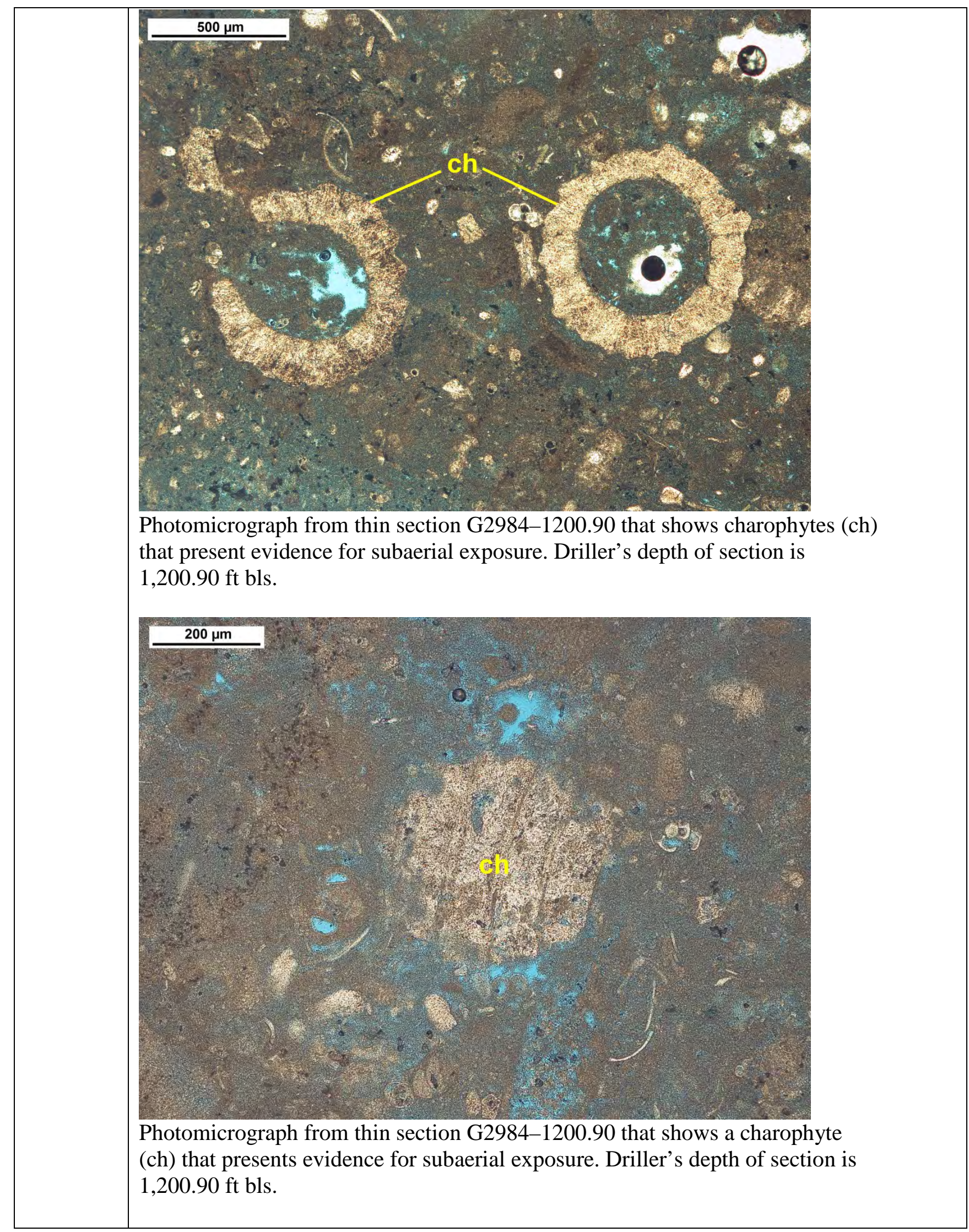




\begin{tabular}{|c|c|}
\hline $\begin{array}{l}\text { obi } \\
\text { depth: } \\
\text { 1,205.3- } \\
\text { 1,206.4 ft } \\
\text { bls } \\
\text { Driller's } \\
\text { depth: } \\
\text { 1,204.0- } \\
\text { 1,205.1 ft } \\
\text { bls }\end{array}$ & 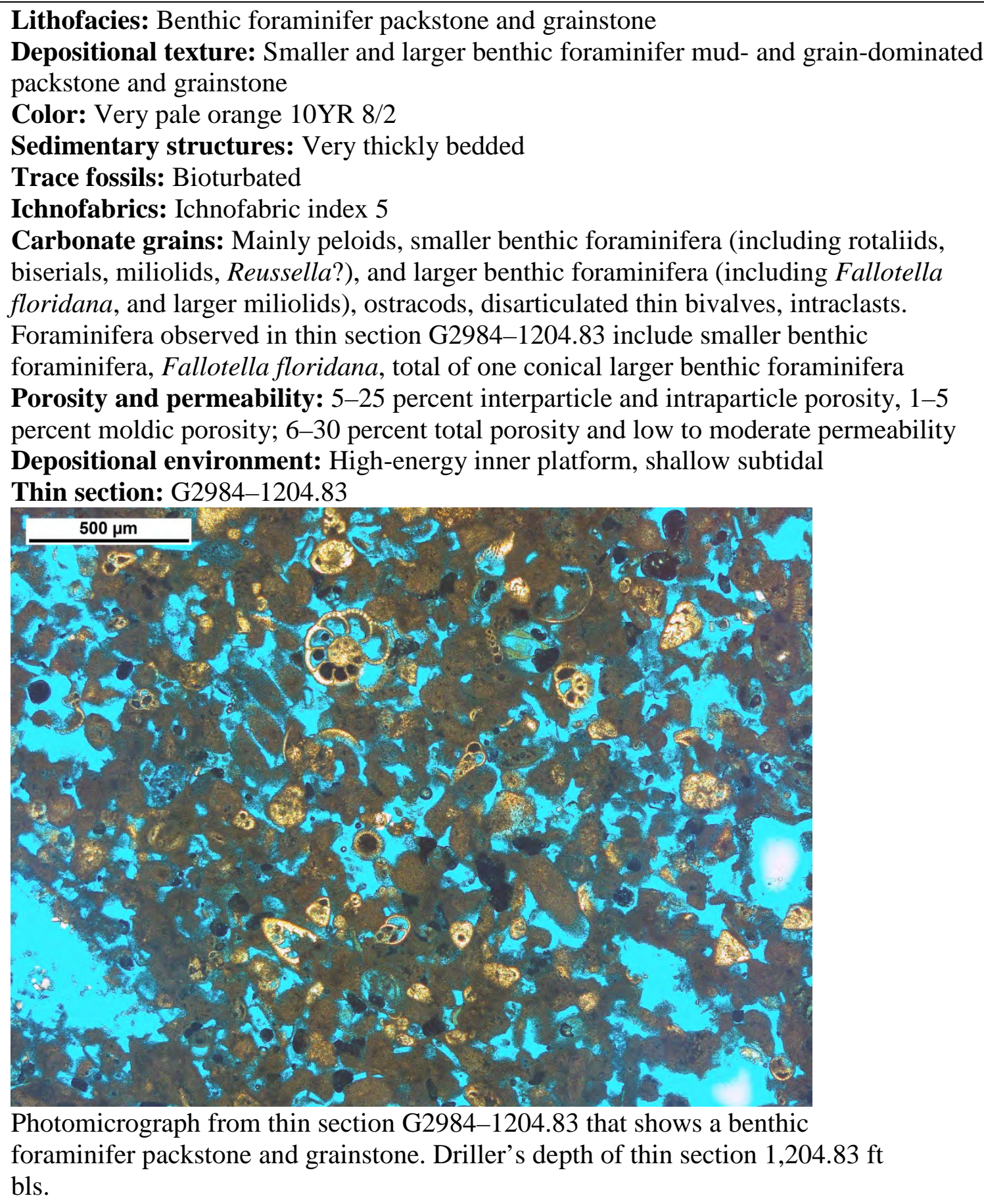 \\
\hline $\begin{array}{l}\text { obi } \\
\text { depth: } \\
1,206.4- \\
1,206.9 \mathrm{ft} \\
\text { bls } \\
\text { Driller's } \\
\text { depth: }\end{array}$ & $\begin{array}{l}\text { Lithofacies: Benthic foraminifer wackestone and packstone } \\
\text { Depositional texture: Smaller benthic and larger foraminifer mud-dominated packstone } \\
\text { Color: Very pale orange } 10 \mathrm{YR} 8 / 2 \text { with minor pale yellowish brown } 10 \mathrm{YR} 6 / 2 \text { paper- } \\
\text { thin, wispy laminations } \\
\text { Sedimentary structures: Thickly bedded } \\
\text { Trace fossils: Burrow mottled } \\
\text { Ichnofabrics: Ichnofabric index } 5 \\
\text { Carbonate grains: Mainly peloids, smaller benthic foraminifera (including rotaliids), }\end{array}$ \\
\hline
\end{tabular}




\begin{tabular}{|c|c|}
\hline $\begin{array}{l}1,205.1- \\
1,205.6 \mathrm{ft} \\
\text { bls }\end{array}$ & $\begin{array}{l}\text { larger benthic foraminifera (including Fallotella cookei, Fallotella floridana), ostracods } \\
\text { Porosity and permeability: } 1-15 \text { percent interparticle and intraparticle porosity; } 1-15 \\
\text { percent total porosity and relatively low permeability } \\
\text { Depositional environment: Low-energy inner platform, shallow subtidal }\end{array}$ \\
\hline $\begin{array}{l}\text { obi } \\
\text { depth: } \\
\text { 1,206.9- } \\
\text { 1,207.4 ft } \\
\text { bls } \\
\text { Driller's } \\
\text { depth: } \\
\text { 1,205.6- } \\
\text { 1,206.1 ft } \\
\text { bls }\end{array}$ & $\begin{array}{l}\text { Cycle type: Top type II cycle } \\
\text { Lithofacies: Benthic foraminifer mudstone and wackestone } \\
\text { Depositional texture: Smaller benthic foraminifer mudstone and wackestone } \\
\text { Color: Very pale orange } 10 \text { YR } 8 / 2 \\
\text { Sedimentary structures: Medium bedded, fenestral fabric } \\
\text { Trace fossils: Rhizoliths } 0.5-1.0-\mathrm{mm} \text { inner tubule diameter } \\
\text { Ichnofabrics: Ichnofabric index } 2 \\
\text { Ichnofacies: Psilonichnus } \\
\text { Carbonate grains: Peloids and smaller benthic foraminifera } \\
\text { Porosity and permeability: } 1-5 \text { percent interparticle and intraparticle porosity, } 2-3 \\
\text { percent root mold porosity, } 1 \text { percent fenestrae porosity; } 1-9 \text { percent total porosity and } \\
\text { relatively low permeability } \\
\text { Depositional environment: Low-energy restricted inner platform, tidal flat } \\
\text { Comments: Top of generally fining upward peritidal cycle at } 1,206.9 \mathrm{ft} \text { bls (obi depth) } \\
\text { and } 1,205.6 \text { ft bls (driller's depth) }\end{array}$ \\
\hline $\begin{array}{l}\text { obi } \\
\text { depth: } \\
\text { 1,207.4- } \\
\text { 1,209.0 ft } \\
\text { bls } \\
\text { Driller's } \\
\text { depth: } \\
\text { 1,206.1- } \\
\text { 1,207.8 ft } \\
\text { bls }\end{array}$ & $\begin{array}{l}\text { Lithofacies: Benthic foraminifer wackestone and packstone } \\
\text { Depositional texture: Smaller benthic foraminifer wackestone and mud-dominated } \\
\text { packstone } \\
\text { Color: Very pale orange } 10 \text { YR } 8 / 2 \\
\text { Sedimentary structures: Thickly bedded } \\
\text { Trace fossils: Bioturbated, rhizoliths } \\
\text { Ichnofabrics: Ichnofabric index } 5 \\
\text { Carbonate grains: Mainly smaller benthic foraminifera (including miliolids), peloids, } \\
\text { minor ostracods, larger miliolids, uncommon echinoid spines } \\
\text { Porosity and permeability: } 1-10 \text { percent interparticle and intraparticle porosity, } 1 \\
\text { percent root molds, } 1-2 \text { percent fossil molds; } 3-13 \text { percent total porosity and relatively } \\
\text { low permeability } \\
\text { Depositional environment: Low-energy restricted inner platform, tidal flat }\end{array}$ \\
\hline $\begin{array}{l}\text { obi } \\
\text { depth: } \\
1,209.0- \\
\text { 1,210.0 ft } \\
\text { bls } \\
\text { Driller's } \\
\text { depth: } \\
\text { 1,207.8- } \\
\text { 1,208.8 ft } \\
\text { bls }\end{array}$ & $\begin{array}{l}\text { Lithofacies: Benthic foraminifer packstone and grainstone } \\
\text { Depositional texture: Smaller and larger benthic foraminifer mud- and grain-dominated } \\
\text { packstone and grainstone } \\
\text { Color: Very pale orange } 10 \text { YR } 8 / 2 \\
\text { Sedimentary structures: Medium bedded } \\
\text { Trace fossils: Bioturbated } \\
\text { Ichnofabrics: Ichnofabric index } 5 \\
\text { Carbonate grains: Mainly smaller benthic foraminifera (including rotaliids, biserials, } \\
\text { Reussella?), peloids, and larger benthic foraminifera (including Fallotella floridana, and } \\
\text { larger miliolids), intraclasts, ostracods, fragmented bivalves } \\
\text { Porosity and permeability: } 5-25 \text { percent interparticle and intraparticle porosity, 1-2 } \\
\text { percent moldic porosity; 6-27 percent total porosity and moderate permeability } \\
\text { Depositional environment: High-energy inner platform, shallow subtidal } \\
\text { Comments: Minor rip-up intraclasts along base of interval up to medium pebble size }\end{array}$ \\
\hline
\end{tabular}




\begin{tabular}{|c|c|}
\hline $\begin{array}{l}\text { obi } \\
\text { depth: } \\
1,210.0- \\
\text { 1,211.4 ft } \\
\text { bls } \\
\text { Driller's } \\
\text { depth: } \\
\text { 1,208.8- } \\
\text { 1,210.2 ft } \\
\text { bls }\end{array}$ & $\begin{array}{l}\text { Cycle type: Top type II cycle } \\
\text { Lithofacies: Benthic foraminifer wackestone and packstone } \\
\text { Depositional texture: Smaller benthic foraminifer wackestone and mud-dominated } \\
\text { packstone } \\
\text { Color: Very light gray N8 } \\
\text { Sedimentary structures: Thickly bedded } \\
\text { Trace fossils: Minor rhizoliths with } 0.5 \text {-mm inner tubule diameter } \\
\text { Ichnofabrics: Ichnofabric index } 5 \\
\text { Ichnofacies: Psilonichnus } \\
\text { Carbonate grains: Mainly smaller benthic foraminifera (including miliolids, } \\
\text { Reussella?), peloids, minor ostracods, larger miliolids, uncommon echinoid spines, rare } \\
\text { charophytes? Foraminifera observed in thin section G2984-1210.42 include smaller } \\
\text { benthic foraminifera (thin section fits into this interval) } \\
\text { Diagenesis: Circumgranular curved-plane cracks and vertical joint plane cracks } \\
\text { Porosity and permeability: } 1-10 \text { percent interparticle and intraparticle porosity, } 1 \text { root } \\
\text { molds, } 1-2 \text { percent fossil molds; } 3-13 \text { percent total porosity and relatively low } \\
\text { permeability } \\
\text { Depositional environment: Low-energy restricted inner platform, intertidal to } \\
\text { supratidal } \\
\text { Comments: Highly irregular paleotopography with about } 1 \text { in. of paleorelief probably } \\
\text { related to desiccation (cracks) and karst dissolution during exposure. Top of generally } \\
\text { fining upward peritidal cycle at } 1,210.0 \text { ft bls (obi depth) and 1,208.8 ft bls (driller's } \\
\text { depth) } \\
\text { Thin section: G2984-1210.42 (thin section fits into this interval) }\end{array}$ \\
\hline $\begin{array}{l}\text { obi } \\
\text { depth: } \\
\text { 1,211.4- } \\
\text { 1,214.9 ft } \\
\text { bls } \\
\text { Driller's } \\
\text { depth: } \\
\text { 1,210.2- } \\
\text { 1,213.7 ft } \\
\text { bls }\end{array}$ & $\begin{array}{l}\text { Lithofacies: Benthic foraminifer packstone and grainstone } \\
\text { Depositional texture: Smaller and larger benthic foraminifer mud- and grain-dominated } \\
\text { packstone and grainstone } \\
\text { Color: Very pale orange } 10 \text { YR } 8 / 2 \\
\text { Sedimentary structures: Thickly bedded } \\
\text { Trace fossils: Bioturbated } \\
\text { Ichnofabrics: Ichnofabric index } 5 \\
\text { Carbonate grains: Mainly smaller benthic foraminifera (including rotaliids, biserials, } \\
\text { Reussella?), peloids, and larger benthic foraminifera (including Fallotella floridana, and } \\
\text { larger miliolids), ostracods, fragmented bivalves. Foraminifera observed in thin section } \\
\text { G2984-1213.33 include smaller benthic foraminifera, Fallotella floridana, larger } \\
\text { valvulinids, total of seven conical larger benthic foraminifera } \\
\text { Porosity and permeability: } 5-25 \text { percent interparticle and intraparticle porosity, 1-2 } \\
\text { percent moldic porosity; 6-27 percent total porosity and moderate permeability } \\
\text { Depositional environment: High-energy inner platform, shallow subtidal } \\
\text { Thin section: G2984-1213.33 }\end{array}$ \\
\hline
\end{tabular}




\begin{tabular}{|c|c|}
\hline & 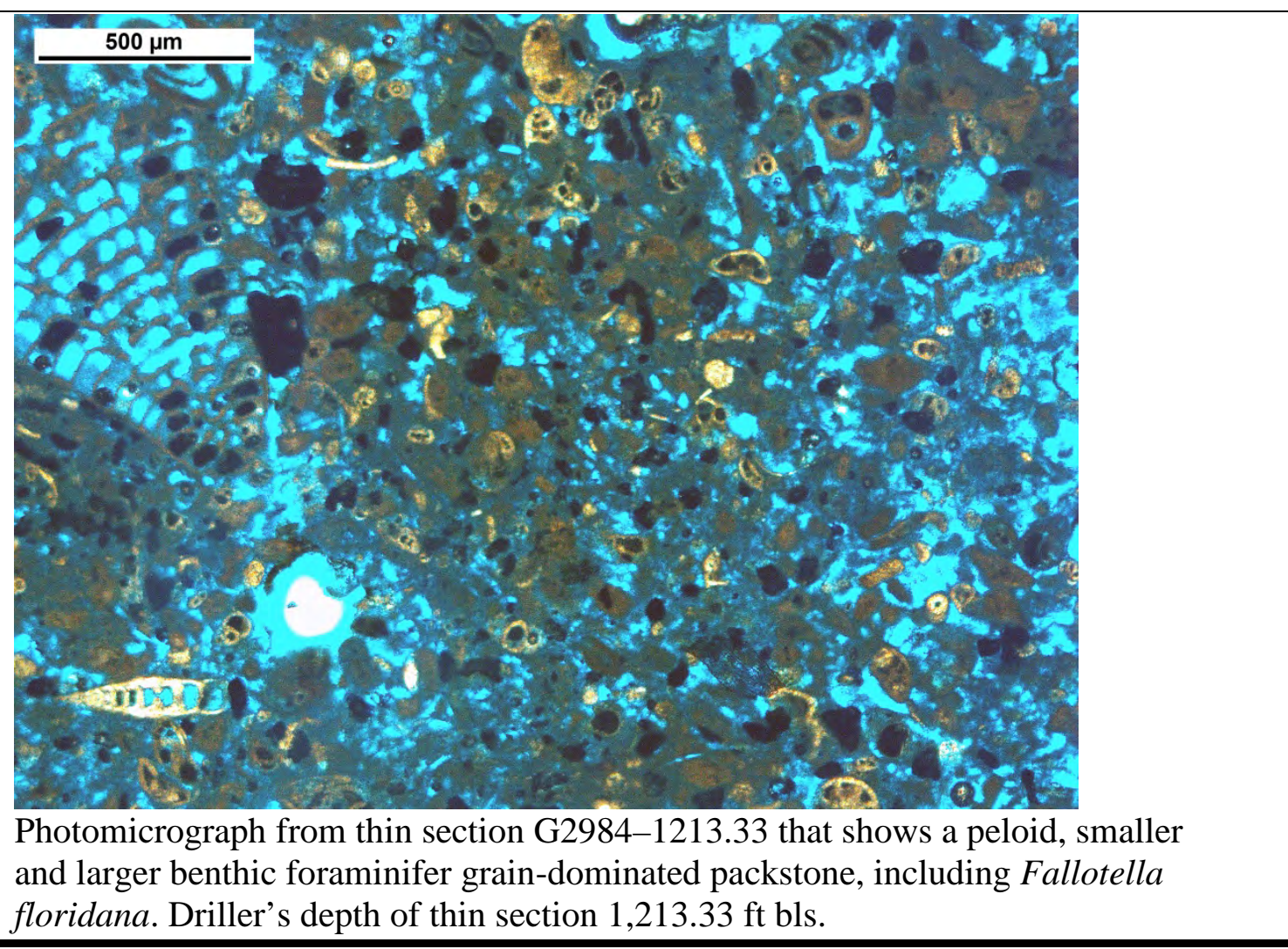 \\
\hline $\begin{array}{l}\text { obi } \\
\text { depth: } \\
\text { 1,214.9- } \\
\text { 1,215.0 ft } \\
\text { bls } \\
\text { Driller's } \\
\text { depth: } \\
\text { 1,213.7- } \\
\text { 1,213.8 ft } \\
\text { bls }\end{array}$ & $\begin{array}{l}\text { Cycle type: Top type II cycle } \\
\text { Lithofacies: Benthic foraminifer mudstone and wackestone } \\
\text { Depositional texture: Smaller benthic foraminifer mudstone and wackestone } \\
\text { Color: Yellow gray 5Y 8/1 (wet) } \\
\text { Sedimentary structures: Thinly bedded, vertical desiccation cracks } \\
\text { Trace fossils: Rhizoliths } 0.5-\mathrm{mm} \text { inner tubule diameter } \\
\text { Ichnofabrics: Ichnofabric index } 2 \\
\text { Ichnofacies: Psilonichnus } \\
\text { Carbonate grains: Peloids and smaller benthic foraminifera } \\
\text { Porosity and permeability: } 1-5 \text { percent interparticle and intraparticle porosity, } 2-3 \\
\text { percent root mold porosity; } 3-8 \text { percent total porosity and relatively low permeability } \\
\text { Depositional environment: Low-energy restricted inner platform, tidal flat } \\
\text { Comments: Top of generally fining upward peritidal cycle at 1,214.9 ft bls (obi depth) } \\
\text { and } 1,213.7 \mathrm{ft} \text { bls (driller's depth) }\end{array}$ \\
\hline $\begin{array}{l}\text { obi } \\
\text { depth: } \\
\text { 1,215.0- } \\
\text { 1,217.8 ft } \\
\text { bls } \\
\text { Driller's } \\
\text { depth: } \\
\text { 1,213.8- } \\
\text { 1,217.7 ft }\end{array}$ & $\begin{array}{l}\text { Lithofacies: Benthic foraminifer wackestone and packstone } \\
\text { Depositional texture: Smaller benthic foraminifer mud-dominated packstone } \\
\text { Color: Very pale orange } 10 \text { YR } 8 / 2 \\
\text { Sedimentary structures: Thickly bedded } \\
\text { Trace fossils: Bioturbated } \\
\text { Ichnofabrics: Ichnofabric index } 5 \\
\text { Carbonate grains: Mainly peloids (most pellets), smaller benthic foraminifera } \\
\text { (including uncommon rotaliids, Reussella?), larger benthic foraminifera (including } \\
\text { Fallotella floridana), ostracods, Neolaganum dalli along base of interval, rare planktic } \\
\text { foraminifera. Foraminifera observed in thin section G2984-1215.31 include smaller }\end{array}$ \\
\hline
\end{tabular}




\begin{tabular}{|c|c|}
\hline bls & $\begin{array}{l}\text { benthic foraminifera, Fallotella floridana, larger valvulinids, Coskinolina floridana?, } \\
\text { total of } 10 \text { conical larger benthic foraminifera } \\
\text { Porosity and permeability: } 1-10 \text { percent interparticle and intraparticle porosity; 1-10 } \\
\text { percent total porosity and relatively low permeability } \\
\text { Depositional environment: Low-energy inner platform, shallow subtidal } \\
\text { Thin section: G2984-1215.31 } \\
\frac{1 \mathrm{~mm}}{6}\end{array}$ \\
\hline $\begin{array}{l}\text { obi } \\
\text { depth: } \\
1,217.8- \\
1,219.3 \mathrm{ft} \\
\text { bls } \\
\text { Driller's } \\
\text { depth: } \\
1,217.7- \\
1,219.6 \mathrm{ft} \\
\text { bls }\end{array}$ & $\begin{array}{l}\text { Lithofacies: Benthic foraminifer wackestone and packstone } \\
\text { Depositional texture: Smaller benthic foraminifer wackestone } \\
\text { Color: Very pale orange 10YR } 8 / 2 \text { and pale yellowish brown 10YR 6/2 very thin } \\
\text { laminations } \\
\text { Sedimentary structures: Thinly bedded } \\
\text { Trace fossils: Burrow mottled; Thalassinoides, Asterosoma } \\
\text { Ichnofabrics: Ichnofabric index } 5 \\
\text { Ichnofacies: Proximal Cruziana } \\
\text { Carbonate grains: Mainly peloids (probably many pellets), smaller benthic foraminifera } \\
\text { (including common globular-shaped multichambered foraminifera, uncommon rotaliids, } \\
\text { Reussella?), minor intraclasts, larger benthic foraminifera (including Fallotella cookei, } \\
\text { Fallotella floridana), ostracods, rare planktic foraminifera. Foraminifera observed in thin } \\
\text { section G2984-1218.92 include smaller benthic foraminifera, Fallotella floridana, larger } \\
\text { valvulinids, Arenagula sp., total of four conical larger benthic foraminifera } \\
\text { Porosity and permeability: 1-7 percent interparticle and intraparticle porosity; } 1-7 \\
\text { percent total porosity and relatively low permeability } \\
\text { Depositional environment: Low-energy restricted lagoon or inner platform, shallow } \\
\text { subtidal } \\
\text { Comments: Abrupt lithofacies shift at top of interval at 1,217.8 ft bls (obi depth) and }\end{array}$ \\
\hline
\end{tabular}




\begin{tabular}{|c|c|}
\hline & 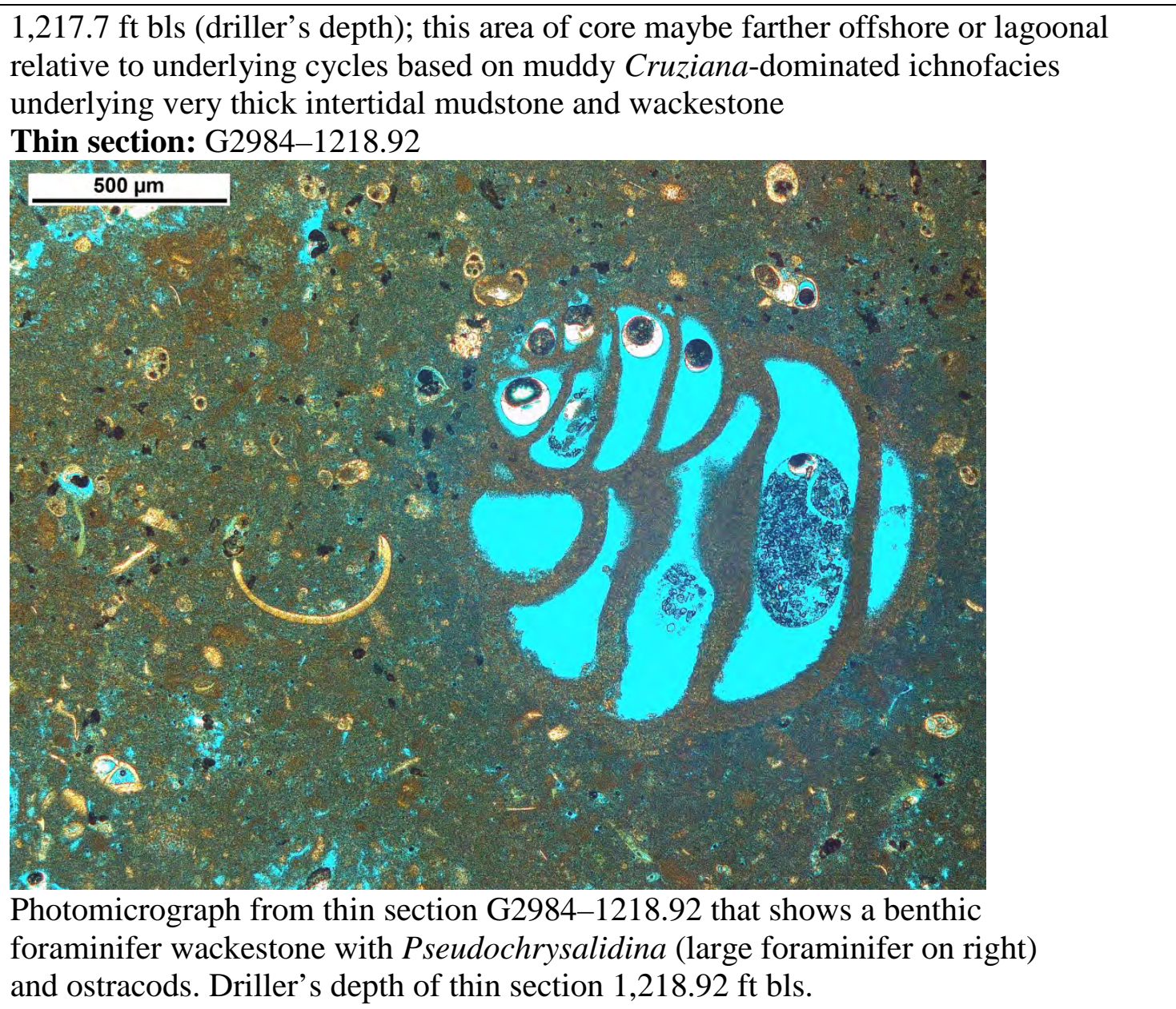 \\
\hline $\begin{array}{l}\text { obi } \\
\text { depth: } \\
\text { 1,219.3- } \\
\text { 1,219.4 ft } \\
\text { bls } \\
\text { Driller's } \\
\text { depth: } \\
\text { 1,219.6- } \\
\text { 1,219.7 ft } \\
\text { bls }\end{array}$ & $\begin{array}{l}\text { Cycle type: Top type II cycle } \\
\text { Lithofacies: Benthic foraminifer mudstone and wackestone } \\
\text { Depositional texture: Smaller benthic foraminifer mudstone and wackestone } \\
\text { Color: Yellow gray } 5 Y \text { Y } 1 \text { (wet) } \\
\text { Sedimentary structures: Thinly bedded } \\
\text { Trace fossils: Rhizoliths } 0.5-1.5-m m \text { inner tubule diameter } \\
\text { Ichnofabrics: Ichnofabric index } 2 \\
\text { Ichnofacies: Psilonichnus } \\
\text { Carbonate grains: Peloids and smaller benthic foraminifera } \\
\text { Porosity and permeability: } 1-5 \text { percent interparticle and intraparticle porosity, } 2-7 \\
\text { percent root mold porosity; } 3-12 \text { percent total porosity and relatively low permeability } \\
\text { Depositional environment: Low-energy restricted inner platform, tidal flat } \\
\text { Comments: Top of generally fining upward peritidal cycle at 1,219.3 ft bls (obi depth) } \\
\text { and } 1,219.6 \mathrm{ft} \text { bls (driller's depth) }\end{array}$ \\
\hline $\begin{array}{l}\text { obi } \\
\text { depth: } \\
1,219.4- \\
1,219.6 \mathrm{ft} \\
\text { bls }\end{array}$ & $\begin{array}{l}\text { Lithofacies: Benthic foraminifer wackestone and packstone } \\
\text { Depositional texture: Smaller benthic foraminifer wackestone } \\
\text { Color: Very pale orange 10YR 8/2 and pale yellowish brown 10YR 6/2 very thin } \\
\text { laminations } \\
\text { Sedimentary structures: Thinly bedded } \\
\text { Trace fossils: Burrow mottled; Thalassinoides }\end{array}$ \\
\hline
\end{tabular}




\begin{tabular}{|c|c|}
\hline $\begin{array}{l}\text { Driller's } \\
\text { depth: } \\
1,219.7- \\
1,219.9 \mathrm{ft} \\
\text { bls }\end{array}$ & $\begin{array}{l}\text { Ichnofabrics: Ichnofabric index } 5 \\
\text { Ichnofacies: Proximal Cruziana } \\
\text { Carbonate grains: Mainly peloids (probably mainly pellets), smaller benthic } \\
\text { foraminifera (including common globular-shaped multichambered foraminifera, } \\
\text { uncommon rotaliids, Reussella?), minor larger benthic foraminifera (including Fallotella } \\
\text { cookei, Fallotella floridana), ostracods, rare planktic foraminifera } \\
\text { Porosity and permeability: } 1-7 \text { percent interparticle and intraparticle porosity; 1-7 } \\
\text { percent total porosity and relatively low permeability } \\
\text { Depositional environment: Low-energy inner platform, shallow subtidal }\end{array}$ \\
\hline $\begin{array}{l}\text { obi } \\
\text { depth: } \\
\text { 1,219.6- } \\
\text { 1,219.8 ft } \\
\text { bls } \\
\text { Driller's } \\
\text { depth: } \\
\text { 1,219.9- } \\
\text { 1,220.1 ft } \\
\text { bls }\end{array}$ & $\begin{array}{l}\text { Cycle type: Top type II cycle } \\
\text { Lithofacies: Benthic foraminifer mudstone and wackestone } \\
\text { Depositional texture: Smaller benthic foraminifer mudstone and wackestone } \\
\text { Color: Yellow gray } 5 Y \text { Y } 1 \text { (wet) } \\
\text { Sedimentary structures: Thinly bedded } \\
\text { Trace fossils: Rhizoliths } 0.5-1.5-\mathrm{mm} \text { inner tubule diameter } \\
\text { Ichnofabrics: Ichnofabric index } 2 \\
\text { Ichnofacies: Psilonichnus } \\
\text { Carbonate grains: Peloids and smaller benthic foraminifera, minor gastropods } \\
\text { Porosity and permeability: } 1-5 \text { percent interparticle and intraparticle porosity, } 2-7 \\
\text { percent root mold porosity; } 3-12 \text { percent total porosity and relatively low permeability } \\
\text { Depositional environment: Low-energy restricted inner platform, tidal flat } \\
\text { Comments: Top of generally fining upward peritidal cycle at 1,219.6 ft bls (obi depth) } \\
\text { and } 1,219.9 \mathrm{ft} \text { bls (driller's depth) }\end{array}$ \\
\hline $\begin{array}{l}\text { obi } \\
\text { depth: } \\
\text { 1,219.8- } \\
\text { 1,220.1 ft } \\
\text { bls } \\
\text { Driller's } \\
\text { depth: } \\
\text { 1,220.1- } \\
\text { 1,220.4 ft } \\
\text { bls }\end{array}$ & $\begin{array}{l}\text { Lithofacies: Benthic foraminifer wackestone and packstone } \\
\text { Depositional texture: Smaller benthic foraminifer wackestone } \\
\text { Color: Very pale orange 10YR 8/2 and pale yellowish brown 10YR 6/2 very thin } \\
\text { laminations } \\
\text { Sedimentary structures: Thinly bedded } \\
\text { Trace fossils: Burrow mottled; Thalassinoides, Rhizocorallium? } \\
\text { Ichnofabrics: Ichnofabric index } 5 \\
\text { Ichnofacies: Proximal Cruziana } \\
\text { Carbonate grains: Mainly peloids (probably many pellets), smaller benthic foraminifera } \\
\text { (including common globular-shaped multichambered foraminifera, uncommon rotaliids, } \\
\text { Reussella?), minor larger benthic foraminifera (including Fallotella cookei, Fallotella } \\
\text { floridana), ostracods, rare planktic foraminifera } \\
\text { Porosity and permeability: } 1-7 \text { percent interparticle and intraparticle porosity; 1-7 } \\
\text { percent total porosity and relatively low permeability } \\
\text { Depositional environment: Low-energy inner platform, shallow subtidal }\end{array}$ \\
\hline $\begin{array}{l}\text { obi } \\
\text { depth: } \\
1,220.1- \\
1,221.0 \mathrm{ft} \\
\text { bls } \\
\text { Driller's } \\
\text { depth: }\end{array}$ & $\begin{array}{l}\text { Lithofacies: Benthic foraminifer wackestone and packstone } \\
\text { Depositional texture: Smaller benthic foraminifer, intraclast wackestone and mud- } \\
\text { dominated packstone } \\
\text { Color: Very pale orange } 10 \text { YR } 8 / 2 \\
\text { Sedimentary structures: Medium bedded } \\
\text { Trace fossils: Bioturbated } \\
\text { Ichnofabrics: Ichnofabric index } 5 \\
\text { Carbonate grains: Mainly peloids (probably many micritized unidentified skeletal }\end{array}$ \\
\hline
\end{tabular}




\begin{tabular}{|c|c|}
\hline $\begin{array}{l}1,220.4- \\
1,221.3 \mathrm{ft} \\
\text { bls }\end{array}$ & $\begin{array}{l}\text { grains), smaller benthic foraminifera, and intraclasts } \\
\text { Porosity and permeability: } 1-15 \text { percent interparticle and intraparticle porosity; 1-15 } \\
\text { percent total porosity and relatively low permeability } \\
\text { Depositional environment: Low-energy inner platform, shallow subtidal }\end{array}$ \\
\hline $\begin{array}{l}\text { obi } \\
\text { depth: } \\
\text { 1,221.0- } \\
\text { 1,221.2 ft } \\
\text { bls } \\
\text { Driller's } \\
\text { depth: } \\
\text { 1,221.3- } \\
\text { 1,221.5 ft } \\
\text { bls }\end{array}$ & $\begin{array}{l}\text { Cycle type: Top type II cycle } \\
\text { Lithofacies: Benthic foraminifer mudstone and wackestone } \\
\text { Depositional texture: Smaller benthic foraminifer mudstone and wackestone } \\
\text { Color: Very pale orange } 10 \text { YR } 8 / 2 \\
\text { Sedimentary structures: Very thinly bedded } \\
\text { Trace fossils: Rhizoliths } 0.5-1.0-\mathrm{mm} \text { inner tubule diameter with some bifurcating } \\
\text { Ichnofabrics: Ichnofabric index } 5 \\
\text { Ichnofacies: Psilonichnus } \\
\text { Carbonate grains: Unidentified skeletal grains and peloids, minor gastropods } \\
\text { Porosity and permeability: } 1-10 \text { percent interparticle and intraparticle porosity, } 2-7 \\
\text { percent root mold porosity; } 3-17 \text { percent total porosity and relatively low permeability } \\
\text { Depositional environment: Low-energy restricted inner platform, tidal flat } \\
\text { Comments: Top of generally fining upward peritidal cycle at } 1,221.0 \mathrm{ft} \text { bls (obi depth) } \\
\text { and } 1,222.0 \mathrm{ft} \text { bls (driller's depth) }\end{array}$ \\
\hline $\begin{array}{l}\text { obi } \\
\text { depth: } \\
1,221.2- \\
\text { 1,221.4 ft } \\
\text { bls } \\
\text { Driller's } \\
\text { depth: } \\
\text { 1,221.5- } \\
\text { 1,221.7 ft } \\
\text { bls }\end{array}$ & $\begin{array}{l}\text { Lithofacies: Benthic foraminifer wackestone and packstone } \\
\text { Depositional texture: Smaller benthic foraminifer wackestone and mud-dominated } \\
\text { packstone } \\
\text { Color: Very pale orange } 10 \mathrm{YR} 8 / 2 \\
\text { Sedimentary structures: Very thinly bedded } \\
\text { Trace fossils: Bioturbated } \\
\text { Ichnofabrics: Ichnofabric index } 5 \\
\text { Carbonate grains: Mainly peloids (probably many micritized unidentified skeletal } \\
\text { grains), smaller benthic foraminifera (including very minor small rotaliids), Neolaganum } \\
\text { dalli } \\
\text { Porosity and permeability: } 1-15 \text { percent interparticle and intraparticle porosity; } 1-15 \\
\text { percent total porosity and relatively low permeability } \\
\text { Depositional environment: Low-energy inner platform, shallow subtidal }\end{array}$ \\
\hline $\begin{array}{l}\text { obi } \\
\text { depth: } \\
\text { 1,221.4- } \\
\text { 1,221.6 ft } \\
\text { bls } \\
\text { Driller's } \\
\text { depth: } \\
\text { 1,221.7- } \\
\text { 1,221.9 ft } \\
\text { bls }\end{array}$ & $\begin{array}{l}\text { Cycle type: Top type II cycle } \\
\text { Lithofacies: Benthic foraminifer mudstone and wackestone } \\
\text { Depositional texture: Smaller benthic foraminifer mudstone and wackestone } \\
\text { Color: Very pale orange } 10 \text { YR } 8 / 2 \\
\text { Sedimentary structures: Very thinly bedded, very minor fenestral fabric } \\
\text { Trace fossils: Rhizoliths } 0.5-1.0 \text {-mm inner tubule diameter with some bifurcating } \\
\text { Ichnofabrics: Ichnofabric index } 5 \\
\text { Ichnofacies: Psilonichnus } \\
\text { Carbonate grains: Unidentified skeletal grains and peloids, minor gastropods } \\
\text { Porosity and permeability: 1-10 percent interparticle and intraparticle porosity, } 2-7 \\
\text { percent root mold porosity; } 3-17 \text { percent total porosity and relatively low permeability } \\
\text { Depositional environment: Low-energy restricted inner platform, tidal flat } \\
\text { Comments: Top of generally fining upward peritidal cycle at } 1,221.4 \mathrm{ft} \text { bls (obi depth) } \\
\text { and } 1,221.7 \mathrm{ft} \text { bls (driller's depth) }\end{array}$ \\
\hline
\end{tabular}




\begin{tabular}{|c|c|}
\hline $\begin{array}{l}\text { obi } \\
\text { depth: } \\
\text { 1,221.6- } \\
\text { 1,225.6 ft } \\
\text { bls } \\
\text { Driller's } \\
\text { depth: } \\
\text { 1,221.9- } \\
\text { 1,224.1 ft } \\
\text { bls }\end{array}$ & 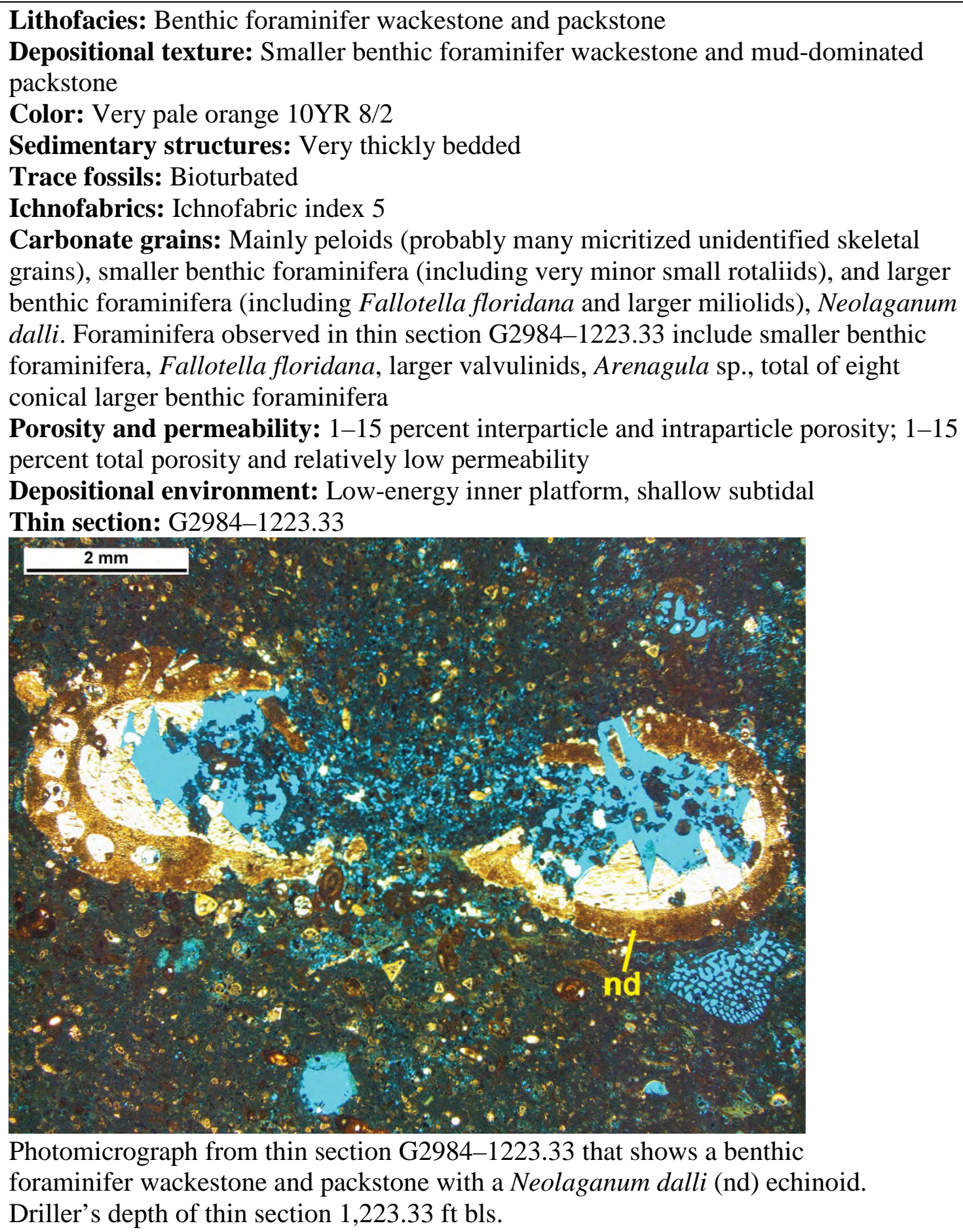 \\
\hline $\begin{array}{l}\text { obi } \\
\text { depth: } \\
1,225.6- \\
1,226.5 \mathrm{ft} \\
\text { bls } \\
\text { Driller's }\end{array}$ & $\begin{array}{l}\text { Lithofacies: Benthic foraminifer packstone and grainstone } \\
\text { Depositional texture: Smaller foraminifer mud- and grain-dominated packstone and } \\
\text { grainstone } \\
\text { Color: Very pale orange } 10 \text { YR } 8 / 2 \\
\text { Sedimentary structures: Medium bedded } \\
\text { Trace fossils: Bioturbated } \\
\text { Ichnofabrics: Ichnofabric index } 5\end{array}$ \\
\hline
\end{tabular}




\begin{tabular}{|c|c|}
\hline $\begin{array}{l}\text { depth: } \\
1,224.1- \\
1,225.0 \mathrm{ft} \\
\text { bls }\end{array}$ & $\begin{array}{l}\text { Carbonate grains: Mainly unidentified small grains, smaller benthic foraminifera, } \\
\text { intraclasts } \\
\text { Porosity and permeability: } 1-15 \text { percent interparticle and intraparticle porosity; } 1-15 \\
\text { percent total porosity and low permeability } \\
\text { Depositional environment: High-energy inner platform, shallow subtidal }\end{array}$ \\
\hline $\begin{array}{l}\text { obi } \\
\text { depth: } \\
\text { 1,226.5- } \\
\text { 1,226.55 } \\
\text { ft bls } \\
\text { Driller's } \\
\text { depth: } \\
\text { 1,225.0- } \\
\text { 1,225.05 } \\
\text { ft bls }\end{array}$ & $\begin{array}{l}\text { Cycle type: Top type I cycle } \\
\text { Lithofacies: Microbial laminite } \\
\text { Depositional texture: Microbial-laminite packstone } \\
\text { Color: Interlaminated very pale orange } 10 \mathrm{YR} 8 / 2 \text { and pale yellowish brown } 10 \mathrm{YR} 6 / 2 \\
\text { and dusky yellowish brown } 10 \mathrm{YR} 2 / 2 \text { organic laminations } \\
\text { Sedimentary structures: Thinly laminated } \\
\text { Carbonate grains: Mainly peloids and smaller benthic foraminifera, minor larger } \\
\text { benthic foraminifera (including Fallotella floridana) } \\
\text { Accessory grains: Organic paper-thin laminations } \\
\text { Porosity and permeability: } 1-5 \text { percent interparticle and intraparticle porosity, and } \\
\text { relatively low permeability } \\
\text { Depositional environment: Low-energy restricted inner platform, tidal flat } \\
\text { Comments: Top of generally fining upward peritidal cycle at } 1,226.5 \mathrm{ft} \text { bls (obi depth) } \\
\text { and 1,225.05 ft bls (driller's depth) }\end{array}$ \\
\hline $\begin{array}{l}\text { obi } \\
\text { depth: } \\
\text { 1,226.55- } \\
\text { 1,227.05 } \\
\text { ft bls } \\
\text { Driller's } \\
\text { depth: } \\
\text { 1,225.05- } \\
\text { 1,225.55 } \\
\text { ft bls }\end{array}$ & $\begin{array}{l}\text { Lithofacies: Benthic foraminifer packstone and grainstone } \\
\text { Depositional texture: Smaller foraminifer mud- and grain-dominated packstone and } \\
\text { grainstone } \\
\text { Color: Very pale orange } 10 \mathrm{YR} 8 / 2 \\
\text { Sedimentary structures: Medium bedded } \\
\text { Trace fossils: Bioturbated } \\
\text { Ichnofabrics: Ichnofabric index } 5 \\
\text { Carbonate grains: Mainly unidentified small grains, smaller benthic foraminifera, } \\
\text { intraclasts, Neolaganum dalli } \\
\text { Porosity and permeability: } 1-15 \text { percent interparticle and intraparticle porosity; } 1-15 \\
\text { percent total porosity and low permeability } \\
\text { Depositional environment: High-energy inner platform, shallow subtidal }\end{array}$ \\
\hline $\begin{array}{l}\text { obi: } \\
\text { 1,227.05- } \\
1,227.1 \mathrm{ft} \\
\text { bls } \\
\text { Driller's } \\
\text { depth: } \\
1,225.55- \\
1,225.6 \mathrm{ft} \\
\text { bls }\end{array}$ & $\begin{array}{l}\text { Cycle type: Top type I cycle } \\
\text { Lithofacies: Microbial laminite } \\
\text { Depositional texture: Microbial laminite-packstone } \\
\text { Color: Interlaminated very pale orange 10YR } 8 / 2 \text { and pale yellowish brown } 10 \mathrm{YR} 6 / 2 \\
\text { Sedimentary structures: Thinly laminated } \\
\text { Carbonate grains: Mainly peloids and smaller benthic foraminifera } \\
\text { Porosity and permeability: } 1-5 \text { percent interparticle and intraparticle porosity, and } \\
\text { relatively low permeability } \\
\text { Depositional environment: Low-energy restricted inner platform, tidal flat } \\
\text { Comments: Top of generally fining upward peritidal cycle at 1,227.05 ft bls (obi depth) } \\
\text { and 1,225.55 ft bls (driller's depth) }\end{array}$ \\
\hline $\begin{array}{l}\text { obi } \\
\text { depth: } \\
1,227.1-\end{array}$ & $\begin{array}{l}\text { Lithofacies: Benthic foraminifer packstone and grainstone } \\
\text { Depositional texture: Smaller and larger benthic foraminifer mud- and grain-dominated } \\
\text { packstone }\end{array}$ \\
\hline
\end{tabular}




\begin{tabular}{|c|c|}
\hline $\begin{array}{l}\text { 1,229.4 ft } \\
\text { bls } \\
\text { Driller's } \\
\text { depth: } \\
\text { 1,225.6- } \\
\text { 1,227.9 ft } \\
\text { bls }\end{array}$ & 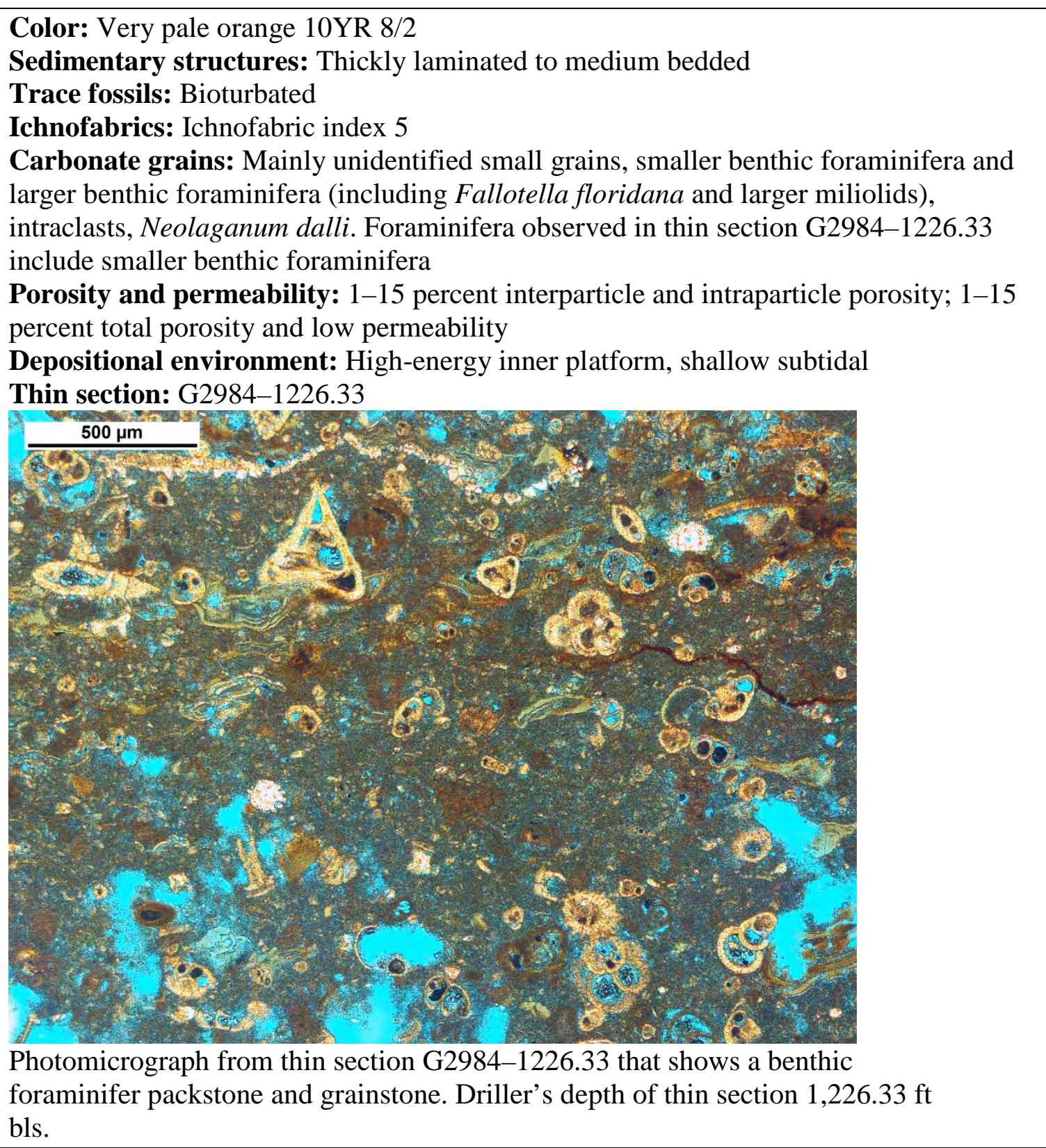 \\
\hline $\begin{array}{l}\text { obi } \\
\text { depth: } \\
\text { 1,229.4- } \\
\text { 1,230.5 ft } \\
\text { bls } \\
\text { Driller's } \\
\text { depth: } \\
\text { 1,227.9- } \\
\text { 1,229.0 ft } \\
\text { bls }\end{array}$ & $\begin{array}{l}\text { Lithofacies: Rip-up clast floatstone } \\
\text { Depositional texture: Intraclast floatstone with a smaller benthic foraminifer grain- } \\
\text { dominated packstone and grainstone matrix } \\
\text { Color: Matrix is very pale orange } 10 \text { YR } 8 / 2 \text { and contains monocolored intraclasts either } \\
\text { very pale orange } 10 Y \mathrm{Y} \text { 8/2, pale yellowish brown } 10 \mathrm{YR} 6 / 2 \text {, light gray N7, or medium } \\
\text { light gray N6 } \\
\text { Sedimentary structures: Medium bedded } \\
\text { Trace fossils: Bioturbated } \\
\text { Ichnofabrics: Ichnofabric index } 5 \\
\text { Carbonate grains: Mainly peloids (probably many micritized unidentified skeletal } \\
\text { grains), smaller benthic foraminifera (including rotaliids), and probably intraclasts are } \\
\text { rip-up clasts (up to small cobble-sized clasts that are mainly composed of smaller benthic }\end{array}$ \\
\hline
\end{tabular}




\begin{tabular}{|c|c|}
\hline & $\begin{array}{l}\text { foraminifera wackestone and minor benthic foraminifera grainstone) } \\
\text { Porosity and permeability: } 1-15 \text { percent interparticle and intraparticle porosity; } 1-15 \\
\text { percent total porosity and relatively low permeability } \\
\text { Depositional environment: High-energy event, marine subtidal }\end{array}$ \\
\hline $\begin{array}{l}\text { obi } \\
\text { depth: } \\
1,230.5- \\
\text { 1,230.8 ft } \\
\text { bls } \\
\text { Driller's } \\
\text { depth: } \\
\text { 1,229.0- } \\
\text { 1,229.3 ft } \\
\text { bls }\end{array}$ & $\begin{array}{l}\text { Cycle type: Top type I cycle } \\
\text { Lithofacies: Microbial laminite } \\
\text { Depositional texture: Microbial laminate-packstone } \\
\text { Color: Interlaminated very pale orange 10YR } 8 / 2 \text { and pale yellowish brown } 10 Y R 6 / 2 \\
\text { and dark yellowish brown 10YR } 4 / 2 \text { organic laminations } \\
\text { Sedimentary structures: Thinly laminated to very thinly bedded } \\
\text { Trace fossils: Planolites } \\
\text { Ichnofabrics: Ichnofabric index 1-3 } \\
\text { Ichnofacies: Skolithos } \\
\text { Carbonate grains: Mainly peloids and smaller benthic foraminifera (including rotaliids } \\
\text { and smaller miliolids), larger miliolids, ostracods, very minor Neolaganum dalli } \\
\text { Accessory grains: Organic paper-thin laminations } \\
\text { Porosity and permeability: 1-5 percent interparticle and intraparticle porosity, and } \\
\text { relatively low permeability } \\
\text { Depositional environment: Low-energy restricted inner platform, tidal flat } \\
\text { Comments: Top of generally fining upward peritidal cycle at 1,230.5 ft bls (obi depth) } \\
\text { and } 1,229.0 \mathrm{ft} \text { bls (driller's depth) }\end{array}$ \\
\hline $\begin{array}{l}\text { obi } \\
\text { depth: } \\
\text { 1,230.8- } \\
\text { 1,231.5 ft } \\
\text { bls } \\
\text { Driller's } \\
\text { depth: } \\
\text { 1,229.3- } \\
\text { 1,230.0 ft } \\
\text { bls }\end{array}$ & $\begin{array}{l}\text { Lithofacies: Benthic foraminifer packstone and grainstone } \\
\text { Depositional texture: Smaller and larger benthic foraminifer grain-dominated packstone } \\
\text { and grainstone } \\
\text { Color: Very pale orange 10YR 8/2 } \\
\text { Sedimentary structures: Thinly laminated to medium bedded } \\
\text { Trace fossils: Thalassinoides } \\
\text { Ichnofabrics: Ichnofabric index 1-5 } \\
\text { Ichnofacies: Distal Skolithos } \\
\text { Carbonate grains: Mainly unidentified small grains, smaller benthic foraminifera, and } \\
\text { larger benthic foraminifera (including Fallotella floridana and larger miliolids), echinoid } \\
\text { spines } \\
\text { Porosity and permeability: 5-25 percent interparticle and intraparticle porosity, 1-8 } \\
\text { percent moldic porosity; 6-33 percent total porosity and moderate permeability } \\
\text { Depositional environment: High-energy inner platform, shallow subtidal }\end{array}$ \\
\hline $\begin{array}{l}\text { obi } \\
\text { depth: } \\
\text { 1,231.5- } \\
\text { 1,234.4 ft } \\
\text { bls } \\
\text { Driller's } \\
\text { depth: } \\
\text { 1,230.0- } \\
\text { 1,232.9 ft } \\
\text { bls }\end{array}$ & $\begin{array}{l}\text { Lithofacies: Skeletal floatstone and rudstone } \\
\text { Depositional texture: Bivalve, echinoid floatstone with peloid, benthic foraminifer } \\
\text { mud- and grain-dominated packstone matrix } \\
\text { Color: Very pale orange } 10 Y \mathrm{Y} 8 / 2 \\
\text { Sedimentary structures: Burrow mottled, thickly bedded } \\
\text { Trace fossils: Minor rhizoliths that are probably from marine plants } \\
\text { Ichnofabrics: Ichnofabric index } 5 \\
\text { Carbonate grains: Mainly peloids (probably many micritized unidentified skeletal } \\
\text { grains), mollusks (articulated and disarticulated bivalves and gastropods), Neolaganum } \\
\text { dalli, smaller benthic foraminifera and larger benthic foraminifera (including Fallotella } \\
\text { floridana), dasycladacean algae, intraclasts, very uncommon possible stick-shaped small }\end{array}$ \\
\hline
\end{tabular}




\begin{tabular}{|c|c|}
\hline & $\begin{array}{l}\text { coral } \\
\text { Porosity and permeability: 5-20 percent interparticle and intraparticle porosity, 1-10 } \\
\text { percent moldic porosity; 6-30 percent total porosity and low to moderate permeability } \\
\text { Depositional environment: Low-energy inner platform, shallow subtidal }\end{array}$ \\
\hline $\begin{array}{l}\text { obi } \\
\text { depth: } \\
\text { 1,234.4- } \\
\text { 1,235.4 ft } \\
\text { bls } \\
\text { Driller's } \\
\text { depth: } \\
\text { 1,232.9- } \\
\text { 1,233.9 ft } \\
\text { bls }\end{array}$ & $\begin{array}{l}\text { Cycle type: Top type I cycle } \\
\text { Lithofacies: Autochthonous breccia } \\
\text { Depositional texture: Breccia } \\
\text { Color: Very pale orange 10YR 8/2, pale yellowish brown 10YR 6/2, local very light } \\
\text { gray N8 to medium gray N5 irregular coloring of wackestone clasts possibly due to local } \\
\text { concentration of organic material } \\
\text { Sedimentary structures: Medium bedded, irregular dissolution voids and interparticle } \\
\text { voids infilled with sediment from superjacent unit } \\
\text { Trace fossils: Uncommon semivertical rhizoliths with about } 0.5-1.0 \text {-mm inner tubule } \\
\text { diameter } \\
\text { Carbonate grains: Mainly rounded intraclasts that contain smaller benthic foraminifera } \\
\text { and unidentified skeletal grains } \\
\text { Porosity and permeability: } 1-10 \text { percent interparticle and intraparticle porosity, } 1 \\
\text { percent root-mold porosity; } 2-11 \text { percent total porosity and relatively low permeability } \\
\text { Depositional environment: Low-energy restricted inner platform, supratidal } \\
\text { Comments: Autochthonous breccia that underwent pedogenetic alteration. Probably } \\
\text { several phases of alteration by desiccation and root growth. Top of generally fining } \\
\text { upward peritidal cycle at } 1,234.4 \mathrm{ft} \text { bls (obi depth) and } 1,233.9 \mathrm{ft} \text { bls (driller's depth) } \\
\text { with evidence for karst dissolution during subaerial exposure }\end{array}$ \\
\hline $\begin{array}{l}\text { obi } \\
\text { depth: } \\
\text { 1,235.4- } \\
\text { 1,235.8 ft } \\
\text { bls } \\
\text { Driller's } \\
\text { depth: } \\
\text { 1,233.9- } \\
\text { 1,234.3 ft } \\
\text { bls }\end{array}$ & $\begin{array}{l}\text { Lithofacies: Microbial laminite } \\
\text { Depositional texture: Microbial laminate-packstone } \\
\text { Color: Interlaminated very pale orange 10YR 8/2 and pale yellowish brown 10YR 6/2 } \\
\text { and dark yellowish brown 10YR 4/2 organic laminations } \\
\text { Sedimentary structures: Thinly laminated to very thinly bedded } \\
\text { Trace fossils: Thalassinoides? } \\
\text { Ichnofabrics: Ichnofabric index 1-2 } \\
\text { Carbonate grains: Mainly peloids and smaller benthic foraminifera (including rotaliids } \\
\text { and smaller miliolids), larger miliolids, ostracods, very minor Neolaganum dalli } \\
\text { Porosity and permeability: 1-5 percent interparticle and intraparticle porosity, and } \\
\text { relatively low permeability } \\
\text { Depositional environment: Low-energy restricted inner platform, tidal flat }\end{array}$ \\
\hline $\begin{array}{l}\text { obi } \\
\text { depth: } \\
\text { 1,235.8- } \\
\text { 1,238.5 ft } \\
\text { bls } \\
\text { Driller's } \\
\text { depth: } \\
\text { 1,234.3- } \\
\text { 1,237.1 ft } \\
\text { bls }\end{array}$ & $\begin{array}{l}\text { Lithofacies: Benthic foraminifer packstone and grainstone } \\
\text { Depositional texture: Smaller and larger benthic foraminifer grain-dominated packstone } \\
\text { and grainstone } \\
\text { Color: Very pale orange } 10 \text { YR } 8 / 2 \\
\text { Sedimentary structures: Burrow mottled, thickly bedded } \\
\text { Trace fossils: None identified } \\
\text { Ichnofabrics: Ichnofabric index } 5 \\
\text { Carbonate grains: Mainly peloids (probably many micritized unidentified skeletal } \\
\text { grains), smaller benthic foraminifera and larger benthic foraminifera (including } \\
\text { Fallotella floridana and larger miliolids), very minor Neolaganum dalli } \\
\text { Porosity and permeability: } 5-25 \text { percent interparticle and intraparticle porosity, 1-3 }\end{array}$ \\
\hline
\end{tabular}




\begin{tabular}{|c|c|}
\hline & $\begin{array}{l}\text { cent moldic porosity; } 6 \text {-28 percent total porosity and moderate permeability } \\
\text { positional environment: High-energy inner platform, shallow subtidal }\end{array}$ \\
\hline $\begin{array}{l}\text { obi } \\
\text { depth: } \\
\text { 1,238.5- } \\
\text { 1,239.3 ft } \\
\text { bls } \\
\text { Driller's } \\
\text { depth: } \\
\text { 1,237.1- } \\
\text { 1,237.9 ft } \\
\text { bls }\end{array}$ & $\begin{array}{l}\text { Cycle type: Top type IV cycle } \\
\text { Lithofacies: Benthic foraminifer mudstone and wackestone } \\
\text { Depositional texture: Smaller benthic foraminifer mudstone and wackestone } \\
\text { Color: Very light gray N8 to light gray N7 } \\
\text { Sedimentary structures: Medium bedded } \\
\text { Trace fossils: Thalassinoides, Psilonichnus } \\
\text { Ichnofabrics: Ichnofabric index } 5 \\
\text { Ichnofacies: Thalassinoides-dominated Glossifungites } \\
\text { Carbonate grains: Unidentified skeletal grains; uncommon, very small gastropods } \\
\text { Porosity and permeability: 1-10 percent interparticle and intraparticle porosity; } 1-10 \\
\text { percent total porosity and relatively low permeability } \\
\text { Depositional environment: Low-energy restricted inner platform, inner tidal flat } \\
\text { Comments: Top of a firmground and Thalassinoides-dominated Glossifungites } \\
\text { ichnofacies cap at 1,238.5 ft bls (obi depth) and 1,237.1 ft bls (driller’s depth). Infill in } \\
\text { Thalassinoides burrow from superjacent benthic foraminifer grain-dominated packstone } \\
\text { and grainstone interval from 1,238.5 to 1,239.3 ft bls (obi depth) and 1,237.1 to 1,237.9 } \\
\text { ft bls (driller's depth). Burrows and Glossifungites ichnofacies continue downward into } \\
\text { subjacent ostracod mudstone and microbial laminite }\end{array}$ \\
\hline $\begin{array}{l}\text { obi } \\
\text { depth: } \\
\text { 1,239.3- } \\
\text { 1,239.8 ft } \\
\text { bls } \\
\text { Driller's } \\
\text { depth: } \\
\text { 1,237.9- } \\
\text { 1,238.4 ft } \\
\text { bls }\end{array}$ & $\begin{array}{l}\text { Lithofacies: Ostracod mudstone } \\
\text { Depositional texture: Ostracod and smaller benthic foraminifer mudstone } \\
\text { Color: Very light gray N8 to light gray N7 } \\
\text { Sedimentary structures: Thickly bedded, skew-plane and curved-plane desiccation } \\
\text { cracks } \\
\text { Trace fossils: Thalassinoides, rhizoliths with } 0.5-1.0 \text {-mm inner diameter and in some } \\
\text { cases have bifurcating tubules, Psilonichnus } \\
\text { Ichnofabrics: Ichnofabric index } 5 \\
\text { Ichnofacies: Thalassinoides-dominated Glossifungites } \\
\text { Carbonate grains: Minor ostracods and unidentified smaller benthic foraminifera. } \\
\text { Foraminifera observed in thin section G2984-1238.25 include smaller benthic } \\
\text { foraminifera } \\
\text { Porosity and permeability: } 1-3 \text { percent root-mold porosity, } 1 \text { percent desiccation-crack } \\
\text { porosity; 2-4 percent total porosity and relatively low permeability } \\
\text { Depositional environment: Low-energy restricted inner platform, inner tidal flat } \\
\text { Comments: Thalassinoides-dominated Glossifungites ichnofacies associated with } \\
\text { superjacent and subjacent Glossifungites ichnofacies. Infill in Thalassinoides burrow } \\
\text { from overlying interval from 1,238.5 to 1,239.3 ft bls (obi depth) and 1,237.1 to 1,237.9 } \\
\text { ft bls (driller's depth) } \\
\text { Thin section: G2984-1238.25 }\end{array}$ \\
\hline
\end{tabular}




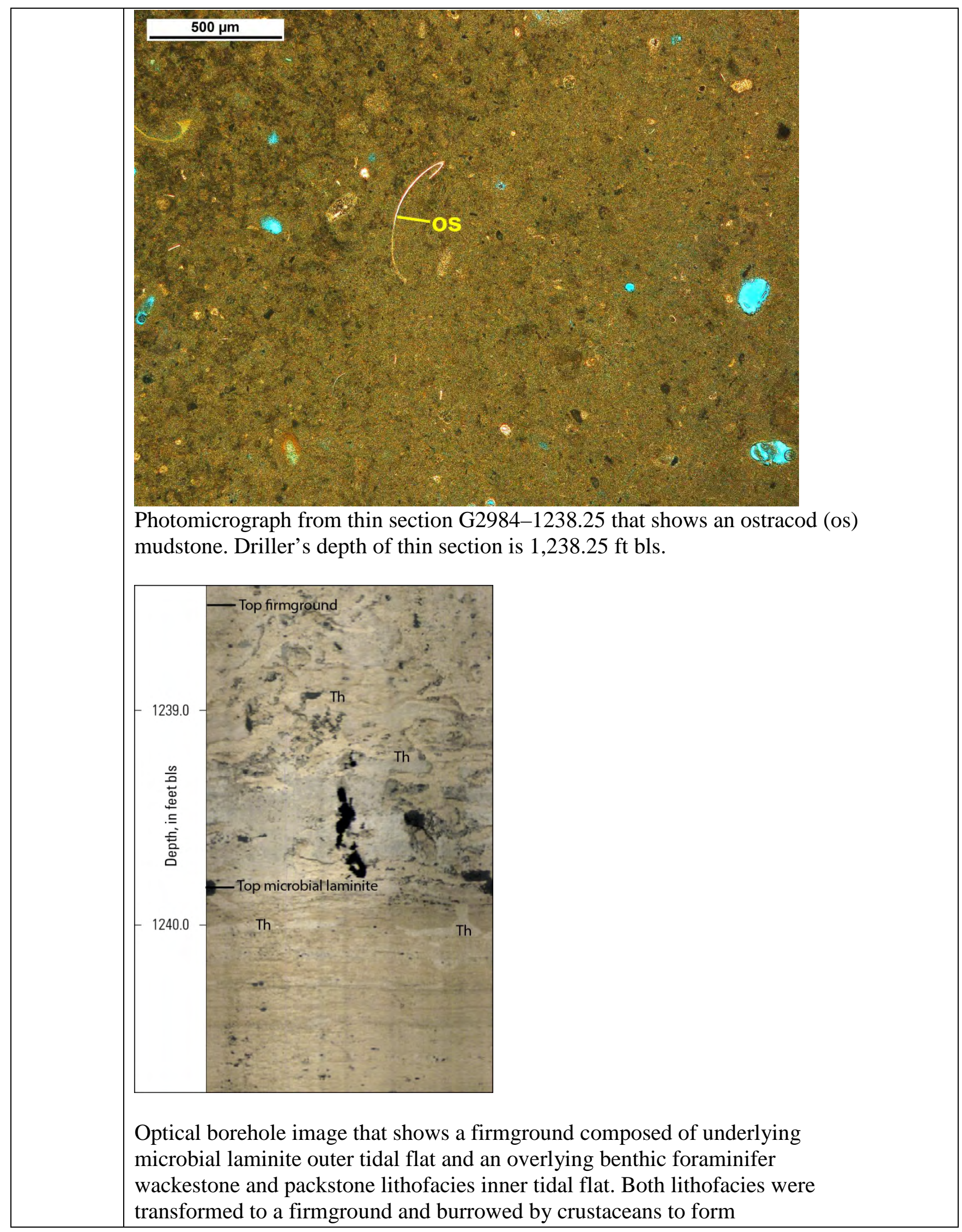




\begin{tabular}{|c|c|}
\hline & $\begin{array}{l}\text { halassinoides (Glossifungites ichnofacies) extending downward from the cycle } \\
\text { p and backfilled with sediment from the overlying cycle. Th is abbreviation } \\
\text { or Thalassinoides. }\end{array}$ \\
\hline $\begin{array}{l}\text { obi } \\
\text { depth: } \\
\text { 1,239.8- } \\
\text { 1,240.5 ft } \\
\text { bls } \\
\text { Driller's } \\
\text { depth: } \\
\text { 1,238.4- } \\
\text { 1,239.1 ft } \\
\text { bls }\end{array}$ & $\begin{array}{l}\text { Lithofacies: Microbial laminite } \\
\text { Depositional texture: Microbial laminite-packstone } \\
\text { Color: Interlaminated very pale orange 10YR } 8 / 2 \text { and pale yellowish brown 10YR 6/2 } \\
\text { Sedimentary structures: Thinly laminated to very thinly bedded } \\
\text { Trace fossils: Thalassinoides } \\
\text { Ichnofabrics: Ichnofabric index 1-2 } \\
\text { Ichnofacies: Thalassinoides-dominated Glossifungites } \\
\text { Carbonate grains: Mainly peloids and smaller benthic foraminifera (including rotaliids } \\
\text { and smaller miliolids), larger miliolids, ostracods } \\
\text { Porosity and permeability: 1-5 percent interparticle and intraparticle porosity, and } \\
\text { relatively low permeability } \\
\text { Depositional environment: Low-energy restricted inner platform, outer tidal flat } \\
\text { Comments: Glossifungites ichnofacies in uppermost part of unit is associated with } \\
\text { superjacent Glossifungites ichnofacies intervals. The uppermost part of this microbial } \\
\text { laminite is a firmground. Infill in Thalassinoides burrow from overlying interval from } \\
1,238.5 \text { to 1,239.3 ft bls (obi depth) and 1,237.1 to 1,237.9 ft bls (driller's depth). }\end{array}$ \\
\hline $\begin{array}{l}\text { obi } \\
\text { depth: } \\
\text { 1,240.5- } \\
\text { 1,243.1 ft } \\
\text { bls } \\
\text { Driller's } \\
\text { depth: } \\
\text { 1,239.1- } \\
\text { 1,241.8 ft } \\
\text { bls }\end{array}$ & $\begin{array}{l}\text { Lithofacies: Benthic foraminifer packstone and grainstone } \\
\text { Depositional texture: Smaller and larger benthic foraminifer grain-dominated packstone } \\
\text { and grainstone } \\
\text { Color: Very pale orange } 10 \text { YR } 8 / 2 \\
\text { Sedimentary structures: Burrow mottled, thickly bedded } \\
\text { Trace fossils: Ophiomorpha } \\
\text { Ichnofabrics: Ichnofabric index } 5 \\
\text { Ichnofacies: Skolithos } \\
\text { Carbonate grains: Mainly peloids (probably many micritized unidentified skeletal } \\
\text { grains), smaller benthic foraminifera and larger benthic foraminifera (including } \\
\text { Fallotella floridana, and larger miliolids), very minor Neolaganum dalli } \\
\text { Porosity and permeability: 5-25 percent interparticle and intraparticle porosity, 1-3 } \\
\text { percent moldic porosity; 6-28 percent total porosity and moderate permeability } \\
\text { Depositional environment: High-energy inner platform, shallow subtidal }\end{array}$ \\
\hline $\begin{array}{l}\text { obi } \\
\text { depth: } \\
\text { 1,243.1- } \\
\text { 1,243.7 ft } \\
\text { bls } \\
\\
\text { Driller's } \\
\text { depth: } \\
\text { 1,241.8- } \\
\text { 1,242.4 ft } \\
\text { bls }\end{array}$ & $\begin{array}{l}\text { Cycle type: Type IV cycle } \\
\text { Lithofacies: Benthic foraminifer wackestone and packstone } \\
\text { Depositional texture: Smaller and larger benthic foraminifer mud- and grain-dominated } \\
\text { packstone } \\
\text { Color: Very pale orange } 10 \text { YR } 8 / 2 \text { matrix, light gray N7 to medium light gray N8 rip-up } \\
\text { intraclasts } \\
\text { Sedimentary structures: Burrow mottled, medium bedded } \\
\text { Trace fossils: Thalassinoides } \\
\text { Ichnofabrics: Ichnofabric index } 5 \\
\text { Ichnofacies: Thalassinoides-dominated Glossifungites } \\
\text { Carbonate grains: Mainly peloids (probably many micritized unidentified skeletal } \\
\text { grains), smaller benthic foraminifera and larger benthic foraminifera (including } \\
\text { Fallotella floridana and larger miliolids), rip-up intraclasts up to small pebble size (rip- }\end{array}$ \\
\hline
\end{tabular}




\begin{tabular}{|c|c|}
\hline & $\begin{array}{l}\text { ups of benthic foraminifer wackestone-possibly total erosion of a pre-existing } \\
\text { underlying benthic foraminifer wackestone) } \\
\text { Porosity and permeability: 5-15 percent interparticle and intraparticle porosity, 1-3 } \\
\text { percent moldic porosity; 6-18 percent total porosity and low permeability } \\
\text { Depositional environment: Low-energy inner platform, shallow subtidal } \\
\text { Comments: Thalassinoides-dominated Glossifungites ichnofacies with burrows infilled } \\
\text { with superjacent benthic foraminifer grain-dominated packstone and grainstone } \\
\text { Comments: Top of a firmground-capped subtidal cycle at 1,243.1 ft bls (obi depth) and } \\
1,241.8 \mathrm{ft} \text { bls (driller's depth) }\end{array}$ \\
\hline $\begin{array}{l}\text { obi } \\
\text { depth: } \\
\text { 1,243.7- } \\
\text { 1,244.0 ft } \\
\text { bls } \\
\text { Driller's } \\
\text { depth: } \\
\text { 1,242.4- } \\
\text { 1,242.7 ft } \\
\text { bls }\end{array}$ & $\begin{array}{l}\text { Cycle type: Top type I cycle } \\
\text { Lithofacies: Microbial laminite } \\
\text { Depositional texture: Microbial laminite-packstone } \\
\text { Color: Interlaminated very pale orange 10YR } 8 / 2 \text { and pale yellowish brown } 10 \mathrm{YR} 6 / 2 \text {, } \\
\text { dark yellowish brown } 10 \mathrm{YR} 4 / 2 \text { organic laminations } \\
\text { Sedimentary structures: Thinly to thickly laminated, horizontal to wavy laminations } \\
\text { Trace fossils: Minor small burrows, Planolites? } \\
\text { Ichnofabrics: Ichnofabric index } 2 \\
\text { Carbonate grains: Mainly peloids and smaller benthic foraminifera (including rotaliids } \\
\text { and smaller miliolids), larger miliolids, ostracods } \\
\text { Accessory grains: Very minor paper-thin organic laminations } \\
\text { Porosity and permeability: } 1-5 \text { percent interparticle and intraparticle porosity, and } \\
\text { relatively low permeability } \\
\text { Depositional environment: Low-energy restricted inner platform, tidal flat } \\
\text { Comments: Cycle top at 1,243.7 ft bls (obi depth) and 1,242.4 ft bls (driller's depth) }\end{array}$ \\
\hline $\begin{array}{l}\text { obi } \\
\text { depth: } \\
1,244.0- \\
1,245.3 \mathrm{ft} \\
\text { bls } \\
\text { Driller's } \\
\text { depth: } \\
\text { 1,242.7- } \\
1,243.7 \mathrm{ft} \\
\text { bls }\end{array}$ & $\begin{array}{l}\text { Lithofacies: Benthic foraminifer packstone and grainstone } \\
\text { Depositional texture: Smaller and larger benthic foraminifer grain-dominated packstone } \\
\text { and grainstone } \\
\text { Color: Very pale orange } 10 \mathrm{YR} 8 / 2 \\
\text { Sedimentary structures: Burrow mottled, medium bedded } \\
\text { Trace fossils: None identified } \\
\text { Ichnofabrics: Ichnofabric index } 5 \\
\text { Carbonate grains: Mainly peloids (probably many micritized unidentified skeletal } \\
\text { grains), smaller benthic foraminifera, and larger benthic foraminifera (including } \\
\text { Fallotella floridana and larger miliolids) } \\
\text { Porosity and permeability: } 5 \text {-25 percent interparticle and intraparticle porosity, 1-3 } \\
\text { percent moldic porosity; 6-28 percent total porosity and moderate permeability } \\
\text { Depositional environment: High-energy inner platform, shallow subtidal }\end{array}$ \\
\hline $\begin{array}{l}\text { obi } \\
\text { depth: } \\
1,245.3- \\
1,246.15 \\
\text { ft bls }\end{array}$ & $\begin{array}{l}\text { Lithofacies: Rip-up clast floatstone } \\
\text { Depositional texture: Intraclast floatstone with matrix of smaller and larger benthic } \\
\text { foraminifer mud- and grain-dominated packstone } \\
\text { Color: Very pale orange 10YR 8/2 matrix, light gray N7 to medium light gray N8 rip-up } \\
\text { intraclasts } \\
\text { Sedimentary structures: Burrow mottled, medium bedded } \\
\text { Trace fossils: None identified } \\
\text { Ichnofabrics: Ichnofabric index } 5\end{array}$ \\
\hline
\end{tabular}




\begin{tabular}{|c|c|}
\hline $\begin{array}{l}1,243.7- \\
1,244.8 \mathrm{ft} \\
\text { bls }\end{array}$ & $\begin{array}{l}\text { Carbonate grains: Mainly peloids (probably many micritized unidentified skeletal } \\
\text { grains), smaller benthic foraminifera and larger benthic foraminifera (including } \\
\text { Fallotella floridana, and larger miliolids), rip-up intraclasts up to medium pebble size } \\
\text { (rip-ups of benthic foraminifer wackestone-possibly total erosion of a pre-existing } \\
\text { micrite-rich intertidal to supratidal lithofacies), very minor Neolaganum dalli } \\
\text { Porosity and permeability: } 5 \text {-15 percent interparticle and intraparticle porosity, 1-3 } \\
\text { percent moldic porosity; } 6-18 \text { percent total porosity and low permeability } \\
\text { Depositional environment: High-energy event, marine subtidal } \\
\text { Comment: Marine erosion of intertidal to supratidal sediments }\end{array}$ \\
\hline $\begin{array}{l}\text { obi } \\
\text { depth: } \\
\text { 1,246.15- } \\
\text { 1,246.4 ft } \\
\text { bls } \\
\text { Driller's } \\
\text { depth: } \\
\text { 1,244.8- } \\
\text { 1,245.0 ft } \\
\text { bls }\end{array}$ & $\begin{array}{l}\text { Cycle type: Top type I cycle } \\
\text { Lithofacies: Microbial laminite } \\
\text { Depositional texture: Microbial laminite-packstone } \\
\text { Color: Interlaminated very pale orange } 10 \mathrm{YR} 8 / 2 \text { and pale yellowish brown } 10 \mathrm{YR} 6 / 2 \\
\text { Sedimentary structures: Thinly to thickly laminated, horizontal laminations } \\
\text { Trace fossils: Thalassinoides } \\
\text { Ichnofabrics: Ichnofabric index } 2-3 \\
\text { Ichnofacies: Distal Cruziana } \\
\text { Carbonate grains: Mainly peloids and smaller benthic foraminifera } \\
\text { Porosity and permeability: } 1-5 \text { percent interparticle and intraparticle porosity, and } \\
\text { relatively low permeability } \\
\text { Depositional environment: Low-energy restricted inner platform, tidal flat } \\
\text { Comments: Top of generally fining upward peritidal cycle at } 1,246.15 \mathrm{ft} \text { bls (obi depth) } \\
\text { and } 1,244.8 \mathrm{ft} \text { bls (driller's depth) }\end{array}$ \\
\hline $\begin{array}{l}\text { Obi } \\
\text { depth: } \\
\text { 1,246.4- } \\
\text { 1,249.3 ft } \\
\text { bls } \\
\text { Driller's } \\
\text { depth: } \\
\text { 1,245.0- } \\
\text { 1,248.7 ft } \\
\text { bls }\end{array}$ & $\begin{array}{l}\text { Cycle type: Intra-type I cycle } \\
\text { Lithofacies: Benthic foraminifer packstone and grainstone } \\
\text { Depositional texture: Smaller and larger benthic foraminifer grain-dominated packstone } \\
\text { and grainstone } \\
\text { Color: Very pale orange } 10 \text { YR } 8 / 2 \\
\text { Sedimentary structures: Burrow mottled, thickly bedded } \\
\text { Trace fossils: Planolites (deposit feeder), Thalassinoides in uppermost } 0.3 \mathrm{ft} \text { of unit } \\
\text { Ichnofabrics: Ichnofabric index } 5 \text { in general, } 2 \text { in uppermost } 0.3 \mathrm{ft} \text { of unit } \\
\text { Ichnofacies: Proximal Cruziana } \\
\text { Carbonate grains: Mainly peloids (probably many micritized unidentified skeletal } \\
\text { grains), smaller benthic foraminifera, and larger benthic foraminifera (including } \\
\text { Fallotella floridana and larger miliolids) } \\
\text { Porosity and permeability: } 5-25 \text { percent interparticle and intraparticle porosity, } 1-3 \\
\text { percent moldic porosity; } 6 \text {-28 percent total porosity and moderate permeability } \\
\text { Depositional environment: High-energy inner platform, shallow subtidal }\end{array}$ \\
\hline $\begin{array}{l}\text { obi } \\
\text { depth: } \\
1,249.3- \\
1,251.2 \mathrm{ft} \\
\text { bls } \\
\text { Driller's }\end{array}$ & $\begin{array}{l}\text { Cycle type: Top type IV cycle } \\
\text { Lithofacies: Benthic foraminifer mudstone and wackestone } \\
\text { Depositional texture: Smaller benthic foraminifer mudstone and wackestone } \\
\text { Color: Very light gray N8, very minor light gray N7 to medium light gray N6 } \\
\text { Sedimentary structures: Thickly bedded } \\
\text { Trace fossils: Thalassinoides (suspension feeder) } \\
\text { Ichnofabrics: Ichnofabric index } 5\end{array}$ \\
\hline
\end{tabular}




\begin{tabular}{|c|c|}
\hline $\begin{array}{l}\text { depth: } \\
1,248.7- \\
1,250.6 \mathrm{ft} \\
\text { bls }\end{array}$ & $\begin{array}{l}\text { Ichnofacies: Glossifungites } \\
\text { Carbonate grains: Mainly smaller benthic foraminifera (including very minor small } \\
\text { rotaliids, smaller miliolids) and ostracods, unidentified skeletal grains, minor } \\
\text { dasycladacean algae. Foraminifera observed in thin section G2984-1249.58 include } \\
\text { smaller benthic foraminifera } \\
\text { Porosity and permeability: } 1-10 \text { percent interparticle and intraparticle porosity; 3-17 } \\
\text { percent total porosity and relatively low permeability } \\
\text { Depositional environment: Low-energy inner platform, shallow subtidal } \\
\text { Comments: Cycle top at top of interval, interval is a firmground and Thalassinoides- } \\
\text { dominated Glossifungites ichnofacies with a top at 1,249.3 ft bls (obi depth) and 1,248.7 } \\
\text { ft bls (driller's depth). Thalassinoides burrows infilled with superjacent benthic } \\
\text { foraminifer packstone and grainstone }\end{array}$ \\
\hline $\begin{array}{l}\text { obi } \\
\text { depth: } \\
\text { 1,251.2- } \\
\text { 1,251.7 ft } \\
\text { bls } \\
\text { Driller's } \\
\text { depth: } \\
\text { 1,250.6- } \\
\text { 1,251.0 ft } \\
\text { bls }\end{array}$ & $\begin{array}{l}\text { Cycle type: Top type I cycle } \\
\text { Lithofacies: Microbial laminite } \\
\text { Depositional texture: Microbial laminite-wackestone to packstone } \\
\text { Color: Interlaminated very pale orange 10YR } 8 / 2 \text { and pale yellowish brown } 10 \text { YR 6/2 } \\
\text { Sedimentary structures: Thinly to thickly laminated, topography-draping laminations } \\
\text { Trace fossils: Semivertical rhizolith tubules } \\
\text { Ichnofabrics: Ichnofabric index } 1 \\
\text { Ichnofacies: Psilonichnus? } \\
\text { Carbonate grains: Mainly peloids, Microcodium prisms, and smaller benthic } \\
\text { foraminifera (including rotaliids), minor larger miliolids, ostracods, very uncommon } \\
\text { globular planktic foraminifera. Foraminifera observed in thin section G2984-1250.77 } \\
\text { include smaller benthic foraminifera } \\
\text { Porosity and permeability: 1-5 percent interparticle and intraparticle porosity, and } \\
\text { relatively low permeability } \\
\text { Depositional environment: Low-energy restricted inner platform, tidal flat } \\
\text { Comments: Top of generally fining upward peritidal cycle at 1,251.5 ft bls (obi depth) } \\
\text { and 1,250.8 ft bls (driller’s depth) } \\
\text { Thin section: G2984-1250.77 }\end{array}$ \\
\hline
\end{tabular}




\begin{tabular}{|c|c|}
\hline & 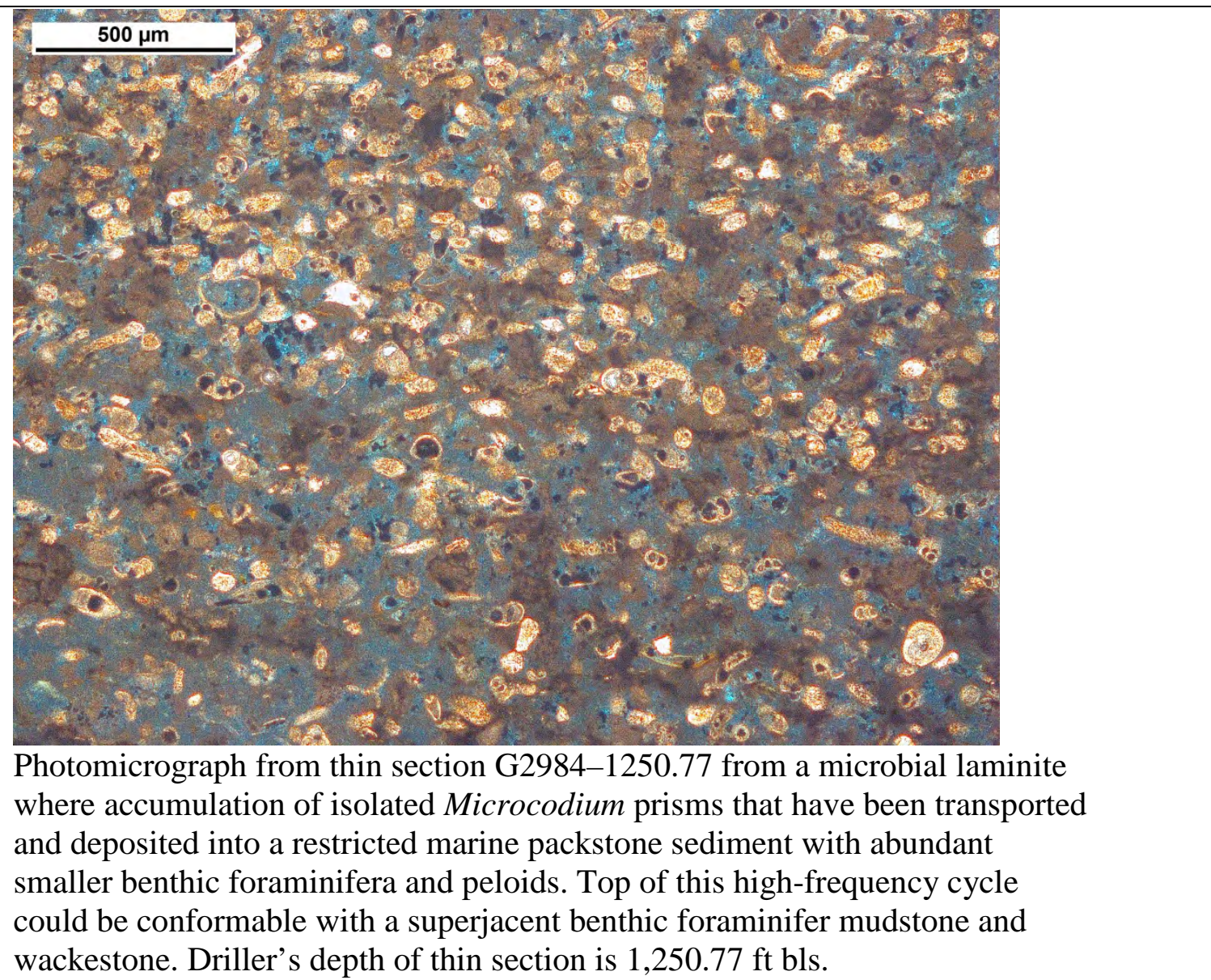 \\
\hline $\begin{array}{l}\text { obi } \\
\text { depth: } \\
\text { 1,251.7- } \\
\text { 1,259.0 ft } \\
\text { bls } \\
\text { Driller's } \\
\text { depth: } \\
\text { 1,251.0- } \\
\text { 1,257.5 ft } \\
\text { bls }\end{array}$ & $\begin{array}{l}\text { Lithofacies: Benthic foraminifer packstone and grainstone } \\
\text { Depositional texture: Smaller and larger benthic foraminifer mud- and grain-dominated } \\
\text { packstone and grainstone } \\
\text { Color: Very pale orange 10YR 8/2 } \\
\text { Sedimentary structures: Burrow mottled, very thickly bedded } \\
\text { Trace fossils: Rhizoliths throughout much of cycle, probably seagrass root molds } \\
\text { Ichnofabrics: Ichnofabric index } 5 \\
\text { Carbonate grains: Mainly peloids (probably many micritized unidentified skeletal } \\
\text { grains), smaller benthic foraminifera (including small rotaliids and smaller miliolids), } \\
\text { and larger benthic foraminifera (including Fallotella floridana and larger miliolids), } \\
\text { uncommon ostracods, echinoid plates } \\
\text { Porosity and permeability: 5-25 percent interparticle and intraparticle porosity, 1-3 } \\
\text { percent moldic porosity; 6-28 percent total porosity and moderate permeability } \\
\text { Depositional environment: High-energy inner platform, shallow subtidal }\end{array}$ \\
\hline $\begin{array}{l}\text { obi } \\
\text { depth: } \\
1,259.0- \\
1,259.8 \mathrm{ft} \\
\text { bls } \\
\text { Driller's }\end{array}$ & $\begin{array}{l}\text { Cycle type: Top type IV cycle } \\
\text { Lithofacies: Benthic foraminifer packstone and grainstone } \\
\text { Depositional texture: Larger and smaller benthic foraminifer mud- to grain-dominated } \\
\text { packstone and grainstone } \\
\text { Color: Very pale orange } 10 \text { YR } 8 / 2 \\
\text { Sedimentary structures: Medium bedded } \\
\text { Trace fossils: Very small Thalassinoides-like burrow? at top of interval (identification }\end{array}$ \\
\hline
\end{tabular}




\begin{tabular}{|c|c|}
\hline $\begin{array}{l}\text { depth: } \\
1,257.5- \\
1,258.3 \mathrm{ft} \\
\text { bls }\end{array}$ & $\begin{array}{l}\text { based on obi borehole wall image only) } \\
\text { Ichnofabrics: Ichnofabric index } 5 \\
\text { Ichnofacies: Glossifungites at uppermost } 0.25 \mathrm{ft} \text { of interval } \\
\text { Carbonate grains: Mainly larger benthic foraminifera (including Fallotella floridana } \\
\text { and larger miliolids), smaller benthic foraminifera, echinoid plates } \\
\text { Porosity and permeability: } 5-20 \text { percent interparticle and intraparticle porosity, 1-3 } \\
\text { percent moldic porosity; 6-23 percent total porosity and low permeability } \\
\text { Depositional environment: High-energy inner platform, shallow subtidal } \\
\text { Comments: Cycle top at top of interval, interval is a firmground and Glossifungites } \\
\text { ichnofacies with a top at 1,259.0 ft bls (obi depth) and 1,258.3 ft bls (driller’s depth). } \\
\text { Very small Thalassinoides-like burrows maybe infilled with superjacent benthic } \\
\text { foraminifer packstone and grainstone. Base of interval appears to be conformable with } \\
\text { subjacent microbial laminite }\end{array}$ \\
\hline $\begin{array}{l}\text { obi } \\
\text { depth: } \\
\text { 1,259.8- } \\
\text { 1,260.0 ft } \\
\text { bls } \\
\text { Driller's } \\
\text { depth: } \\
\text { 1,258.3- } \\
\text { 1,258.5 ft } \\
\text { bls }\end{array}$ & $\begin{array}{l}\text { Cycle top: Top type I cycle } \\
\text { Lithofacies: Microbial laminite } \\
\text { Depositional texture: Microbial laminite_packstone } \\
\text { Color: Interlaminated very pale orange } 10 \text { YR } 8 / 2 \text { and pale yellowish brown } 10 \mathrm{YR} 6 / 2 \\
\text { Sedimentary structures: Thinly to thickly laminated, topography draping laminations, } \\
\text { minor vertical desiccation cracks } \\
\text { Trace fossils: Planolites? } \\
\text { Ichnofabrics: Ichnofabric index } 2 \\
\text { Ichnofacies: Psilonichnus? } \\
\text { Carbonate grains: Mainly peloids and smaller benthic foraminifera } \\
\text { Porosity and permeability: } 1-5 \text { percent interparticle and intraparticle porosity, and } \\
\text { relatively low permeability } \\
\text { Depositional environment: Low-energy restricted inner platform, tidal flat } \\
\text { Comments: Top of generally fining upward peritidal cycle at 1,259.8 ft bls (obi depth) } \\
\text { and } 1,258.3 \text { ft bls (driller's depth) }\end{array}$ \\
\hline $\begin{array}{l}\text { obi } \\
\text { depth: } \\
\text { 1,260.0- } \\
\text { 1,262.5 ft } \\
\text { bls } \\
\text { Driller's } \\
\text { depth: } \\
\text { 1,258.5- } \\
\text { 1,261.1 ft } \\
\text { bls }\end{array}$ & $\begin{array}{l}\text { Lithofacies: Benthic foraminifer packstone and grainstone } \\
\text { Depositional texture: Smaller and larger benthic foraminifer grain-dominated packstone } \\
\text { and grainstone } \\
\text { Color: Very pale orange } 10 \text { YR } 8 / 2 \\
\text { Sedimentary structures: Burrow mottled, thickly bedded } \\
\text { Trace fossils: None identified } \\
\text { Ichnofabrics: Ichnofabric index } 5 \\
\text { Carbonate grains: Mainly peloids (probably many micritized unidentified skeletal } \\
\text { grains), smaller benthic foraminifera (including small rotaliids and smaller miliolids), } \\
\text { larger benthic foraminifera (including Fallotella floridana and larger miliolids), } \\
\text { uncommon ostracods, echinoid plates } \\
\text { Porosity and permeability: } 5-20 \text { percent interparticle and intraparticle porosity, 1-3 } \\
\text { percent moldic porosity; 6-23 percent total porosity and low permeability } \\
\text { Depositional environment: High-energy inner platform, shallow subtidal }\end{array}$ \\
\hline $\begin{array}{l}\text { obi } \\
\text { depth: } \\
1,262.5- \\
1,263.6 \mathrm{ft}\end{array}$ & $\begin{array}{l}\text { Lithofacies: Rip-up clast floatstone } \\
\text { Depositional texture: Intraclast floatstone with matrix of smaller and larger benthic } \\
\text { foraminifer mud- and grain-dominated packstone } \\
\text { Color: Very pale orange } 10 \text { YR } 8 / 2\end{array}$ \\
\hline
\end{tabular}




\begin{tabular}{|c|c|}
\hline $\begin{array}{l}\text { Driller's } \\
\text { depth: } \\
1,261.1- \\
1,262.2 \mathrm{ft} \\
\text { bls }\end{array}$ & $\begin{array}{l}\text { Sedimentary structures: Burrow mottled matrix and intraclasts, medium bedded } \\
\text { Trace fossils: None identified } \\
\text { Ichnofabrics: Ichnofabric index } 5 \\
\text { Carbonate grains: Mainly peloids (probably many micritized unidentified skeletal } \\
\text { grains), smaller benthic foraminifera (including small rotaliids and smaller miliolids), } \\
\text { larger benthic foraminifera (including Fallotella floridana, and larger miliolids), rip-up } \\
\text { intraclasts up to small cobble size (rip-up intraclasts of wackestone and packstone) } \\
\text { Porosity and permeability: 5-15 percent interparticle and intraparticle porosity, 1-3 } \\
\text { percent moldic porosity; 6-18 percent total porosity and low permeability } \\
\text { Depositional environment: High-energy event, marine subtidal } \\
\text { Comments: Intraclasts from transgressive total erosion of micrite-rich cycle cap } \\
\text { associated with subjacent cycle top }\end{array}$ \\
\hline $\begin{array}{l}\text { obi } \\
\text { depth: } \\
\text { 1,263.6- } \\
\text { 1,264.3 ft } \\
\text { bls } \\
\text { Driller's } \\
\text { depth: } \\
\text { 1,262.2- } \\
\text { 1,262.9 ft } \\
\text { bls }\end{array}$ & $\begin{array}{l}\text { Cycle type: Top type I cycle with erosionally removed micrite-rich cycle cap } \\
\text { Lithofacies: Microbial laminite } \\
\text { Depositional texture: Microbial laminite-packstone } \\
\text { Color: Interlaminated very pale orange 10YR 8/2 and pale yellowish brown 10YR 6/2 } \\
\text { Sedimentary structures: Thinly laminated, topography-draping laminations and slight } \\
\text { buildup topography near upper part of unit } \\
\text { Trace fossils: Planolites } \\
\text { Ichnofabrics: Ichnofabric index } 2 \\
\text { Ichnofacies: Psilonichnus } \\
\text { Carbonate grains: Mainly peloids and smaller benthic foraminifera (including rotaliids } \\
\text { and smaller miliolids), larger miliolids, ostracods } \\
\text { Porosity and permeability: 1-5 percent interparticle and intraparticle porosity, and } \\
\text { relatively low permeability } \\
\text { Depositional environment: Low-energy restricted inner platform, tidal flat } \\
\text { Comments: Top of generally fining upward peritidal cycle at 1,263.6 ft bls (obi depth) } \\
\text { and } 1,262.2 \text { ft bls (driller's depth) }\end{array}$ \\
\hline $\begin{array}{l}\text { obi } \\
\text { depth: } \\
\text { 1,264.3- } \\
\text { 1,268.5 ft } \\
\text { bls } \\
\text { Driller's } \\
\text { depth: } \\
\text { 1,262.9- } \\
\text { 1,267.3 ft } \\
\text { bls }\end{array}$ & $\begin{array}{l}\text { Lithofacies: Benthic foraminifer packstone and grainstone } \\
\text { Depositional texture: Smaller and larger benthic foraminifer grain-dominated packstone } \\
\text { and grainstone } \\
\text { Color: Very pale orange 10YR 8/2 } \\
\text { Sedimentary structures: Burrow mottled, very thinly bedded } \\
\text { Trace fossils: Skolithos } \\
\text { Ichnofabrics: Ichnofabric index } 5 \\
\text { Ichnofacies: Distal Skolithos } \\
\text { Carbonate grains: Mainly peloids (probably many micritized unidentified skeletal } \\
\text { grains), smaller benthic foraminifera (including small rotaliids and smaller miliolids), } \\
\text { larger benthic foraminifera (including Fallotella floridana and larger miliolids), } \\
\text { Neolaganum dalli, uncommon ostracods, echinoid plates. Foraminifera observed in thin } \\
\text { section G2984-1266.19 include smaller benthic foraminifera, Fallotella floridana, } \\
\text { Fallotella cookei, total of two conical larger benthic foraminifera } \\
\text { Porosity and permeability: 5-20 percent interparticle and intraparticle porosity, 1-3 } \\
\text { percent moldic porosity; 6-23 percent total porosity and moderate permeability } \\
\text { Depositional environment: High-energy inner platform, shallow subtidal } \\
\text { Thin section: G2984-1266.19 }\end{array}$ \\
\hline
\end{tabular}




\begin{tabular}{|c|c|}
\hline & 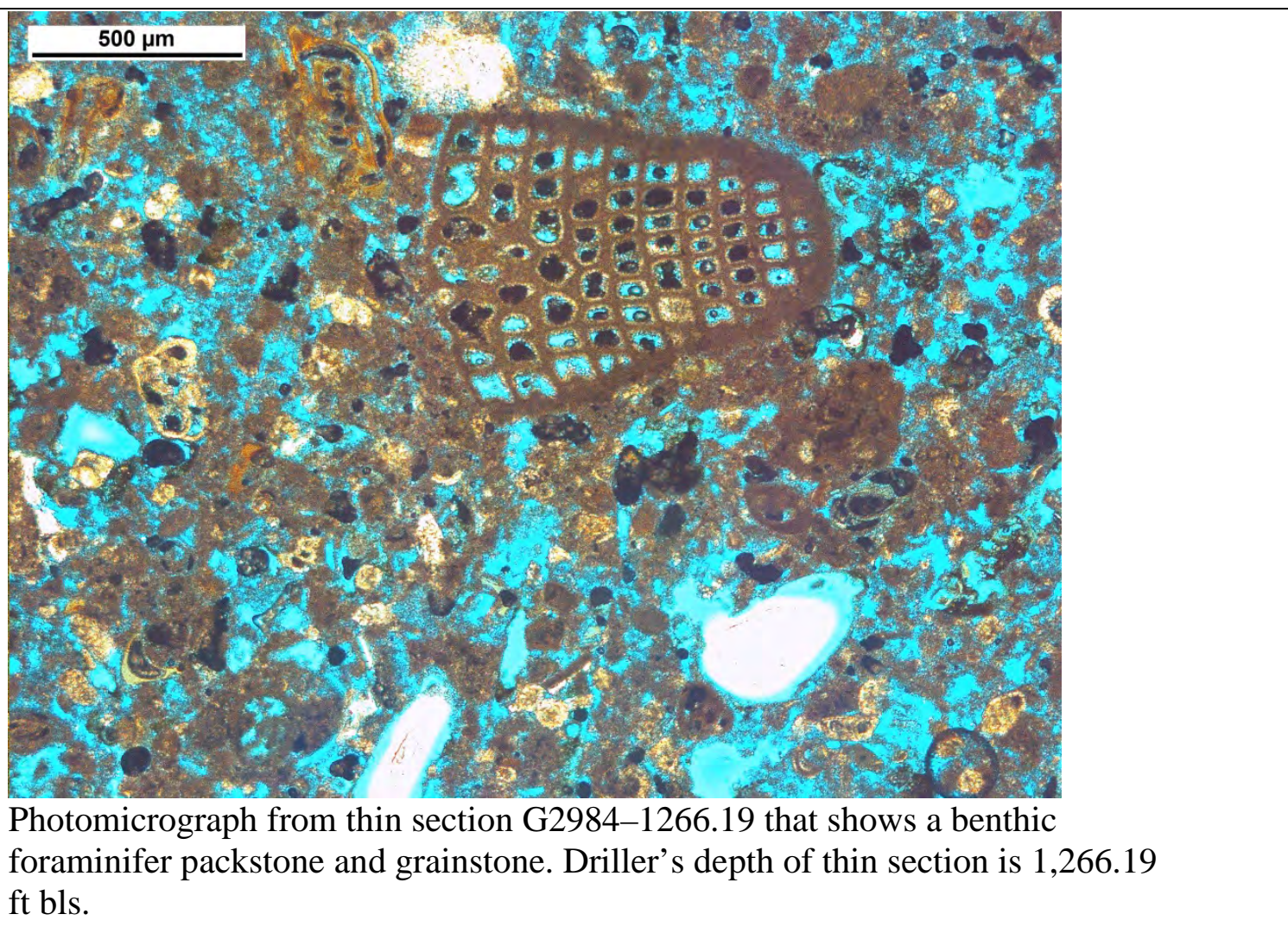 \\
\hline $\begin{array}{l}\text { obi } \\
\text { depth: } \\
\text { 1,268.5- } \\
\text { 1,269.7 ft } \\
\text { bls } \\
\text { Driller's } \\
\text { depth: } \\
\text { 1,267.3- } \\
\text { 1,268.5 ft } \\
\text { bls }\end{array}$ & $\begin{array}{l}\text { Lithofacies: Rip-up clast floatstone } \\
\text { Depositional texture: Intraclast floatstone with matrix of smaller and larger benthic } \\
\text { foraminifer grain-dominated packstone and grainstone } \\
\text { Color: Very pale orange } 10 \text { YR } 8 / 2 \\
\text { Sedimentary structures: Burrow mottled, thickly bedded } \\
\text { Trace fossils: None identified } \\
\text { Ichnofabrics: Ichnofabric index } 5 \\
\text { Carbonate grains: Mainly peloids (probably many micritized unidentified skeletal } \\
\text { grains), smaller benthic foraminifera (including small rotaliids and smaller miliolids), } \\
\text { larger benthic foraminifera (including Fallotella floridana and larger miliolids), rip-up } \\
\text { intraclasts up to medium pebble size (rip-up clasts of underlying benthic foraminifer } \\
\text { mudstone and wackestone-intertidal to supratidal sediment) } \\
\text { Porosity and permeability: 5-15 percent interparticle and intraparticle porosity, 1-3 } \\
\text { percent moldic porosity; 6-18 percent total porosity and low permeability } \\
\text { Depositional environment: High-energy event, marine subtidal. Probably shallow } \\
\text { subtidal transgression }\end{array}$ \\
\hline $\begin{array}{l}\text { obi } \\
\text { depth: } \\
1,269.7- \\
1,270.3 \mathrm{ft} \\
\text { bls } \\
\text { Driller's } \\
\text { depth: }\end{array}$ & $\begin{array}{l}\text { Cycle type: Top-type I cycle } \\
\text { Lithofacies: Autochthonous breccia } \\
\text { Depositional texture: Breccia with clasts of smaller benthic foraminifer mudstone and } \\
\text { wackestone with a matrix of transgressive subtidal marine sediment—-smaller and larger } \\
\text { benthic foraminifer, Microcodium-prism mud-dominated packstone } \\
\text { Color: Very light gray N8, very minor light gray N7 to medium light gray N6 } \\
\text { Sedimentary structures: Very thinly bedded, common desiccation cracks, fenestrae, } \\
\text { autochthonous breccia clasts up to small cobble size }\end{array}$ \\
\hline
\end{tabular}




\begin{tabular}{|c|c|}
\hline $\begin{array}{l}1,268.5- \\
1,268.9 \mathrm{ft} \\
\text { bls }\end{array}$ & $\begin{array}{l}\text { Trace fossils: Rhizoliths as evidenced by } 0.5-0.1 \text {-mm inner diameter tubules and } \\
\text { presence of Microcodium and Microcodium-filled voids (root molds?) up to } 1.5 \mathrm{~cm} \text { in } \\
\text { diameter } \\
\text { Ichnofabrics: Ichnofabric index } 1-2 \\
\text { Ichnofacies: Psilonichnus } \\
\text { Carbonate grains: Mainly smaller benthic foraminifera (including very minor small } \\
\text { rotaliids, smaller miliolids) and ostracods, unidentified skeletal grains, minor larger } \\
\text { benthic foraminifera (including Fallotella floridana), Microcodium. Foraminifera } \\
\text { observed in thin section G2984-1268.83 include smaller benthic foraminifera, Fallotella } \\
\text { floridana, larger valvulinids, total of one conical larger benthic foraminifera } \\
\text { Porosity and permeability: } 1-10 \text { percent interparticle and intraparticle porosity, } 1-3 \\
\text { percent root-mold porosity, } 1-4 \text { percent fenestrae porosity; } 3-17 \text { percent total porosity } \\
\text { and relatively low permeability } \\
\text { Depositional environment: Low-energy restricted inner platform, supratidal } \\
\text { Comments: Top of generally fining upward peritidal cycle at 1,269.7 ft bls (obi depth) } \\
\text { and } 1,268.5 \mathrm{ft} \text { bls (driller's depth). Autochthonous breccia resulting from desiccation and } \\
\text { root stresses } \\
\text { Thin section: G2984-1268.83 }\end{array}$ \\
\hline $\begin{array}{l}\text { obi } \\
\text { depth: } \\
\text { 1,270.3- } \\
\text { 1,270.9 ft } \\
\text { bls } \\
\text { Driller's } \\
\text { depth: } \\
\text { 1,268.9- } \\
\text { 1,269.5 ft } \\
\text { bls }\end{array}$ & $\begin{array}{l}\text { Lithofacies: Microbial laminite } \\
\text { Depositional texture: Microbial laminite-packstone } \\
\text { Color: Interlaminated very pale orange 10YR 8/2 and pale yellowish brown } 10 \mathrm{YR} 6 / 2 \\
\text { Sedimentary structures: Thinly laminated, topography-draping laminations, } \\
\text { interlaminations of organic-rich laminations, and wackestone and mud-dominated } \\
\text { packstone } \\
\text { Carbonate grains: Mainly peloids and smaller benthic foraminifera (including rotaliids } \\
\text { and smaller miliolids), larger miliolids, ostracods, Microcodium. Foraminifera observed } \\
\text { in thin section G2984-1269.04 include smaller benthic foraminifera. Foraminifera } \\
\text { observed in thin section G2984-1269.17 include smaller benthic foraminifera, planktic } \\
\text { foraminifera } \\
\text { Porosity and permeability: } 1-5 \text { percent interparticle and intraparticle porosity, and } \\
\text { relatively low permeability } \\
\text { Depositional environment: Low-energy restricted inner platform, tidal flat } \\
\text { Thin section: G2984-1269.04, G2984-1269.17 }\end{array}$ \\
\hline $\begin{array}{l}\text { obi } \\
\text { depth: } \\
\text { 1,270.9- } \\
\text { 1,274.7 ft } \\
\text { bls } \\
\text { Driller's } \\
\text { depth: } \\
\text { 1,269.5- } \\
\text { 1,273.2 ft } \\
\text { bls }\end{array}$ & $\begin{array}{l}\text { Lithofacies: Benthic foraminifer grain-dominated packstone and grainstone } \\
\text { Depositional texture: Smaller and larger benthic foraminifer grain-dominated packstone } \\
\text { and grainstone } \\
\text { Color: Very pale orange } 10 \text { YR } 8 / 2 \\
\text { Sedimentary structures: Burrow mottled in lower and middle part of unit, very thickly } \\
\text { bedded at base and middle part of unit and thickly laminated and very thinly bedded in } \\
\text { upper part of unit } \\
\text { Trace fossils: None identified } \\
\text { Ichnofabrics: Ichnofabric index } 1-5 \\
\text { Carbonate grains: Mainly peloids (probably many micritized unidentified skeletal } \\
\text { grains), smaller benthic foraminifera (including small rotaliids and smaller miliolids), } \\
\text { larger benthic foraminifera (including Fallotella floridana and larger miliolids), minor } \\
\text { intraclasts up to small pebble size, Neolaganum dalli, uncommon ostracods, thin-shelled }\end{array}$ \\
\hline
\end{tabular}




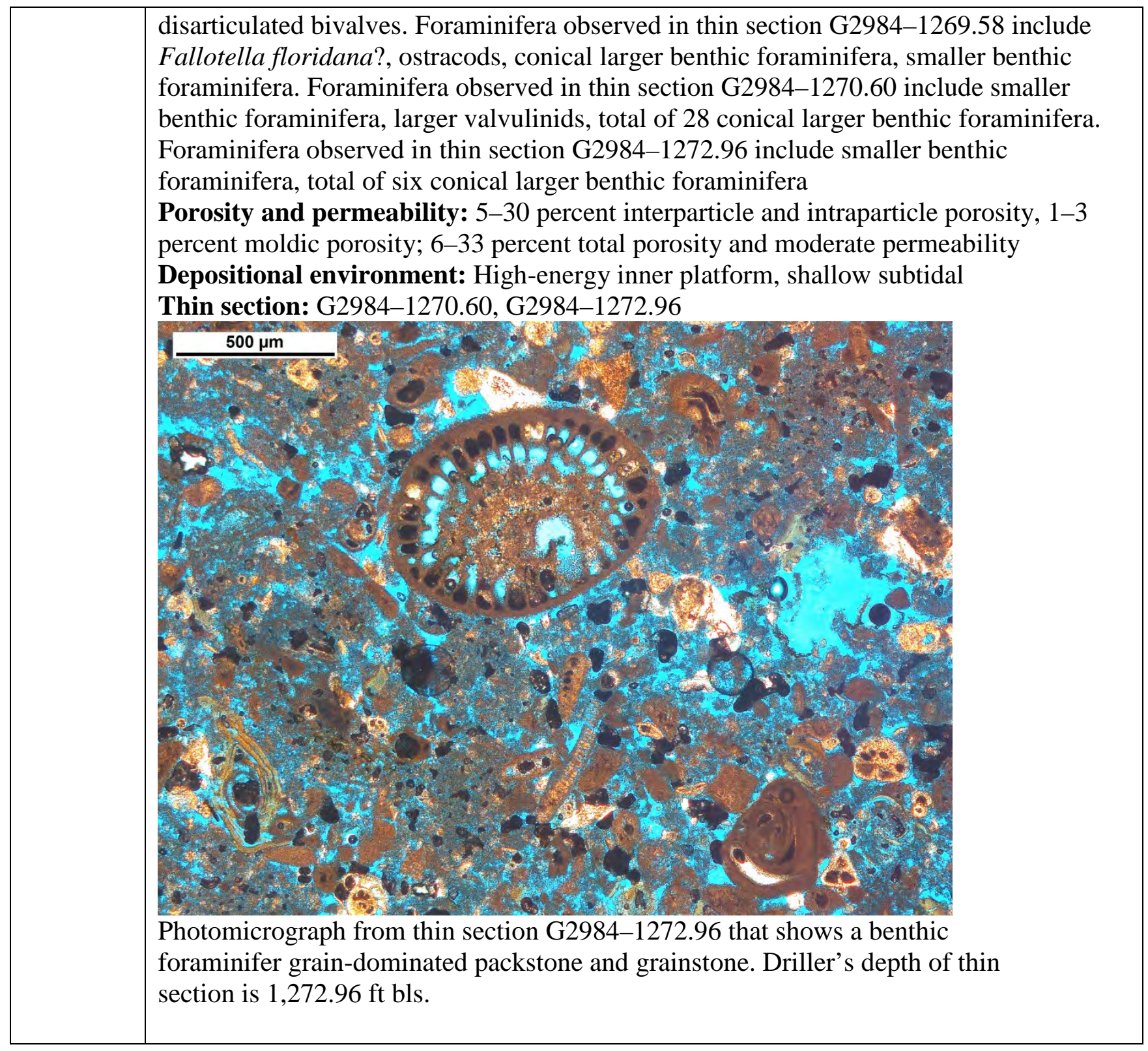




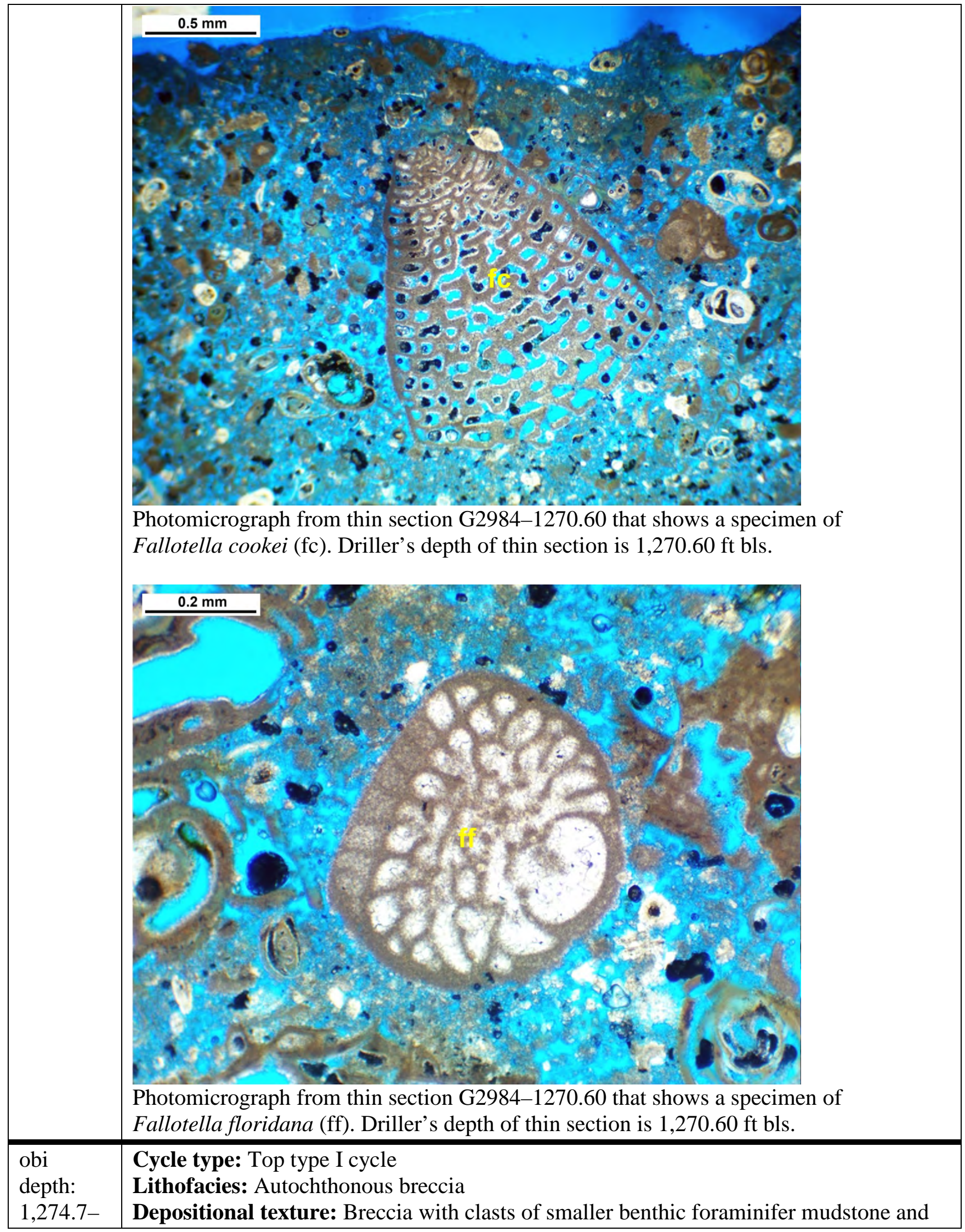




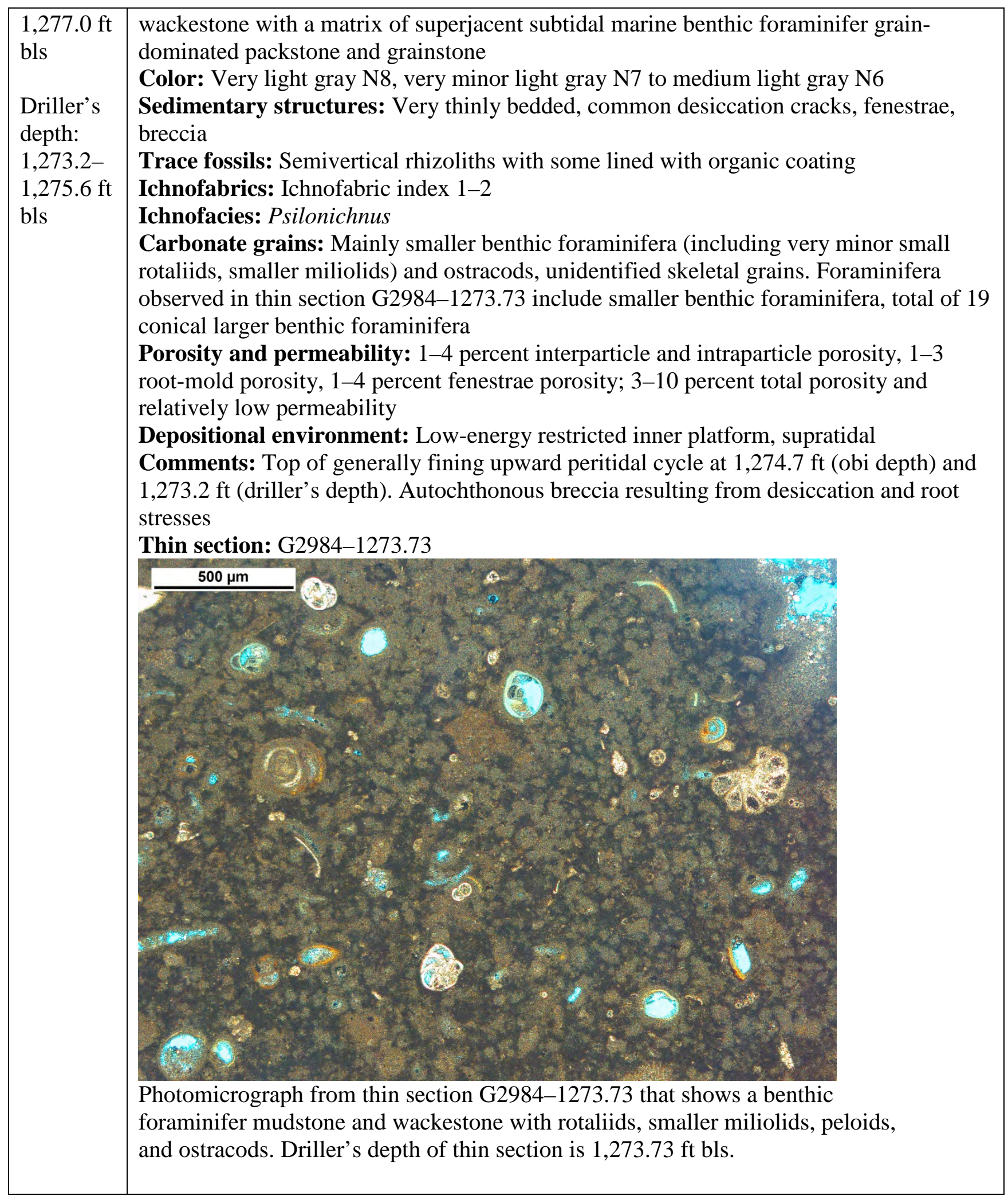




\begin{tabular}{|c|c|}
\hline & 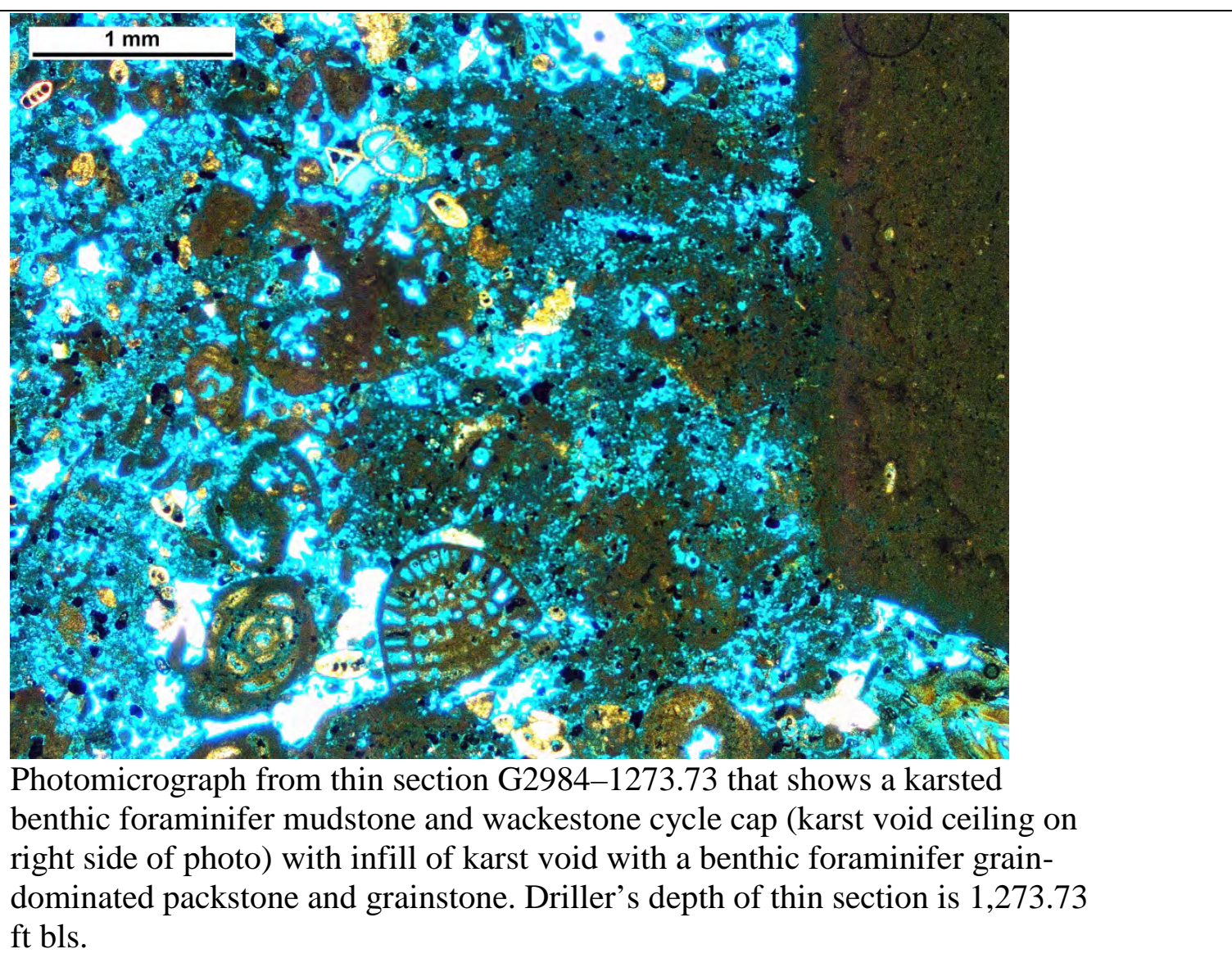 \\
\hline $\begin{array}{l}\text { obi } \\
\text { depth: } \\
\text { 1,277.0- } \\
\text { 1,277.1 ft } \\
\text { bls } \\
\text { Driller's } \\
\text { depth: } \\
\text { 1,275.6- } \\
\text { 1,275.7 ft } \\
\text { bls }\end{array}$ & $\begin{array}{l}\text { Lithofacies: Microbial laminite } \\
\text { Depositional texture: Microbial laminite-packstone } \\
\text { Color: Interlaminated very pale orange } 10 \mathrm{YR} 8 / 2 \text { and pale yellowish brown } 10 \mathrm{YR} 6 / 2 \\
\text { Sedimentary structures: Thinly to thickly laminated, topography-draping laminations } \\
\text { Carbonate grains: Mainly peloids and smaller benthic foraminifera } \\
\text { Porosity and permeability: } 1-10 \text { percent interparticle and intraparticle porosity, and } \\
\text { relatively low permeability } \\
\text { Depositional environment: Low-energy restricted inner platform, tidal flat }\end{array}$ \\
\hline $\begin{array}{l}\text { obi } \\
\text { depth: } \\
\text { 1,277.1- } \\
\text { 1,282.6 ft } \\
\text { bls obi } \\
\text { depth } \\
\text { Driller's } \\
\text { depth: } \\
\text { 1,275.7- } \\
1,281.2 \mathrm{ft}\end{array}$ & $\begin{array}{l}\text { Lithofacies: Benthic foraminifer packstone and grainstone } \\
\text { Depositional texture: Smaller and larger benthic foraminifer grain-dominated packstone } \\
\text { and grainstone with minor smaller and larger benthic foraminifer mud-dominated } \\
\text { packstone } \\
\text { Color: Very pale orange } 10 \text { YR } 8 / 2 \\
\text { Sedimentary structures: Burrow mottled, thickly to very thickly bedded } \\
\text { Trace fossils: Thalassinoides in mud-dominated packstone } \\
\text { Ichnofabrics: Ichnofabric index } 5 \\
\text { Ichnofacies: Distal Skolithos } \\
\text { Carbonate grains: Mainly peloids (probably many micritized unidentified skeletal } \\
\text { grains), smaller benthic foraminifera (including small rotaliids and smaller miliolids), }\end{array}$ \\
\hline
\end{tabular}




\begin{tabular}{|c|c|}
\hline bls & $\begin{array}{l}\text { larger benthic foraminifera (including Fallotella floridana, Coskinolina floridana, and } \\
\text { larger miliolids), minor intraclasts, echinoid plates, uncommon ostracods. Foraminifera } \\
\text { observed in thin section G2984-1281.17 include smaller benthic foraminifera, } \\
\text { Coskinolina floridana, Thomasella? sp., total of nine conical larger benthic foraminifera } \\
\text { Accessory grains: Less than } 1 \text { percent carbonaceous fragments of plants } \\
\text { Porosity and permeability: } 5 \text {-30 percent interparticle and intraparticle porosity, 1-3 } \\
\text { percent moldic porosity; 6-33 percent total porosity and moderate permeability } \\
\text { Depositional environment: High-energy inner platform, shallow subtidal } \\
\text { Thin section: G2984-1281.17 } \\
\quad \mathbf{2 0 0 \mu m}\end{array}$ \\
\hline $\begin{array}{l}\text { obi } \\
\text { depth: } \\
1,282.6- \\
1,282.8 \mathrm{ft} \\
\text { bls } \\
\text { Driller's } \\
\text { depth: } \\
1,281.2- \\
1,281.4 \mathrm{ft} \\
\text { bls }\end{array}$ & $\begin{array}{l}\text { Lithofacies: Rip-up clast floatstone } \\
\text { Depositional texture: Intraclast floatstone with matrix of smaller and larger benthic } \\
\text { foraminifer grain-dominated packstone and grainstone } \\
\text { Color: Very pale orange } 10 Y \mathrm{Y} 8 / 2 \\
\text { Sedimentary structures: Burrow mottled, thinly bedded } \\
\text { Trace fossils: None identified } \\
\text { Ichnofabrics: Ichnofabric index } 5 \\
\text { Carbonate grains: Mainly peloids (probably many micritized unidentified skeletal } \\
\text { grains), smaller benthic foraminifera (including small rotaliids and smaller miliolids), } \\
\text { larger benthic foraminifera (including larger miliolids), minor rip-up intraclasts up to } \\
\text { medium pebble size (rip-ups of underlying benthic foraminifer wackestone-intertidal to } \\
\text { supratidal sediment) } \\
\text { Porosity and permeability: } 5 \text {-15 percent interparticle and intraparticle porosity, 1-3 } \\
\text { percent moldic porosity; 6-18 percent total porosity and low permeability } \\
\text { Depositional environment: High-energy event, marine subtidal. Probably shallow }\end{array}$ \\
\hline
\end{tabular}




\begin{tabular}{|c|c|}
\hline & $\begin{array}{l}\text { marine transgression } \\
\text { Comment: Marine erosion of underlying substrate }\end{array}$ \\
\hline $\begin{array}{l}\text { obi } \\
\text { depth: } \\
\text { 1,282.8- } \\
\text { 1,283.1 ft } \\
\text { bls } \\
\text { Driller's } \\
\text { depth: } \\
\text { 1,281.4- } \\
\text { 1,281.7 ft } \\
\text { bls }\end{array}$ & $\begin{array}{l}\text { Cycle type: Top type II cycle } \\
\text { Lithofacies: Benthic foraminifer wackestone and packstone } \\
\text { Depositional texture: Smaller benthic foraminifer wackestone } \\
\text { Color: Very light gray N8 } \\
\text { Sedimentary structures: Very thinly bedded, common desiccation cracks, fenestrae } \\
\text { Trace fossils: Semivertical rhizoliths? } \\
\text { Ichnofabrics: Ichnofabric index 1-2? } \\
\text { Ichnofacies: Psilonichnus } \\
\text { Carbonate grains: Mainly smaller benthic foraminifera (including very minor small } \\
\text { rotaliids) and ostracods, minor peloids. Foraminifera observed in thin section G2984- } \\
\text { 1281.46 include smaller benthic foraminifera, total of four conical larger benthic } \\
\text { foraminifera } \\
\text { Porosity and permeability: } 1-4 \text { percent interparticle and intraparticle porosity, 1-3 } \\
\text { percent root-mold porosity, 1-4 percent fenestrae porosity; } 3-10 \text { percent total porosity } \\
\text { and relatively low permeability } \\
\text { Depositional environment: Low-energy restricted inner platform, intertidal to } \\
\text { supratidal } \\
\text { Comments: Top of generally fining upward peritidal cycle at } 1,282.8 \mathrm{ft} \text { bls (obi depth) } \\
\text { and } 1,281.4 \mathrm{ft} \text { bls (driller's depth) } \\
\text { Thin section: G2984-1281.46 } \\
\qquad 500 \mu \mathrm{m}\end{array}$ \\
\hline $\begin{array}{l}\text { obi } \\
\text { depth: }\end{array}$ & $\begin{array}{l}\text { Lithofacies: Benthic foraminifer packstone and grainstone } \\
\text { Depositional texture: Smaller and larger benthic foraminifer grain-dominated packstone }\end{array}$ \\
\hline
\end{tabular}




\begin{tabular}{|c|c|}
\hline $\begin{array}{l}\text { 1,283.1- } \\
1,283.8 \mathrm{ft} \\
\text { bls } \\
\text { Driller's } \\
\text { depth: } \\
\text { 1,281.7- } \\
1,282.4 \mathrm{ft} \\
\text { bls }\end{array}$ & $\begin{array}{l}\text { and grainstone } \\
\text { Color: Very pale orange } 10 \mathrm{YR} 8 / 2 \\
\text { Sedimentary structures: Burrow mottled, thinly bedded } \\
\text { Trace fossils: None identified } \\
\text { Ichnofabrics: Ichnofabric index } 5 \\
\text { Carbonate grains: Mainly peloids (probably many micritized unidentified skeletal } \\
\text { grains) and smaller benthic foraminifera (including small rotaliids and smaller miliolids), } \\
\text { minor larger benthic foraminifera (including Fallotella floridana, larger miliolids), } \\
\text { gastropods, uncommon disarticulated bivalves, intraclasts } \\
\text { Porosity and permeability: } 5 \text {-15 percent interparticle and intraparticle porosity, 1-3 } \\
\text { percent moldic porosity; 6-18 percent total porosity and low permeability } \\
\text { Depositional environment: High-energy inner platform, shallow subtidal }\end{array}$ \\
\hline $\begin{array}{l}\text { obi } \\
\text { depth: } \\
\text { 1,283.8- } \\
\text { 1,284.6 ft } \\
\text { bls } \\
\text { Driller's } \\
\text { depth: } \\
\text { 1,282.4- } \\
\text { 1,283.3 ft } \\
\text { bls }\end{array}$ & $\begin{array}{l}\text { Cycle type: Top type II cycle } \\
\text { Lithofacies: Benthic foraminifer wackestone and packstone } \\
\text { Depositional texture: Smaller benthic foraminifer wackestone } \\
\text { Color: Very light gray N8 } \\
\text { Sedimentary structures: Burrow mottled in part, semivertical mudcracks, thickly } \\
\text { laminated to very thinly bedded in part } \\
\text { Trace fossils: Semivertical rhizoliths (some tapering downward and bifurcating } \\
\text { downward) and possible minor worm tubes, both } 0.5 \text {-1-mm inner diameter of tubes } \\
\text { Ichnofabrics: Ichnofabric index } 1-5 \\
\text { Ichnofacies: Psilonichnus } \\
\text { Carbonate grains: Mainly smaller benthic foraminifera (including small rotaliids and a } \\
\text { few small miliolids and biserial foraminifera), minor ostracods, intraclasts. Foraminifera } \\
\text { observed in thin section G2984-1282.50 include smaller benthic foraminifera } \\
\text { Accessory grains: } 1-3 \text { percent small pieces (silt-sized to } 1 \text {-mm diameter or long } \\
\text { roundish, angular, or bladed grains) of black N1 organic material, probably plant debris } \\
\text { Porosity and permeability: } 1 \text {-4 percent interparticle and intraparticle porosity, } 1-3 \\
\text { percent root-mold porosity; } 2-7 \text { percent total porosity and relatively low permeability } \\
\text { Depositional environment: Low-energy restricted inner platform, intertidal to } \\
\text { supratidal } \\
\text { Comments: Lowermost } 0.5 \text { cm of unit is an organic-rich microbial mat overlain by a } \\
\text { very thinly bedded autochthonous breccia, likely due to desiccation and alteration by } \\
\text { roots, very low diversity biota, high environmental stress. Top of generally fining } \\
\text { upward peritidal cycle at } 1,283.8 \mathrm{ft} \text { bls (obi depth) and } 1 \text {,282.4 ft bls (driller's depth) } \\
\text { Thin section: G2984-1282.50 }\end{array}$ \\
\hline
\end{tabular}




\begin{tabular}{|c|c|}
\hline & 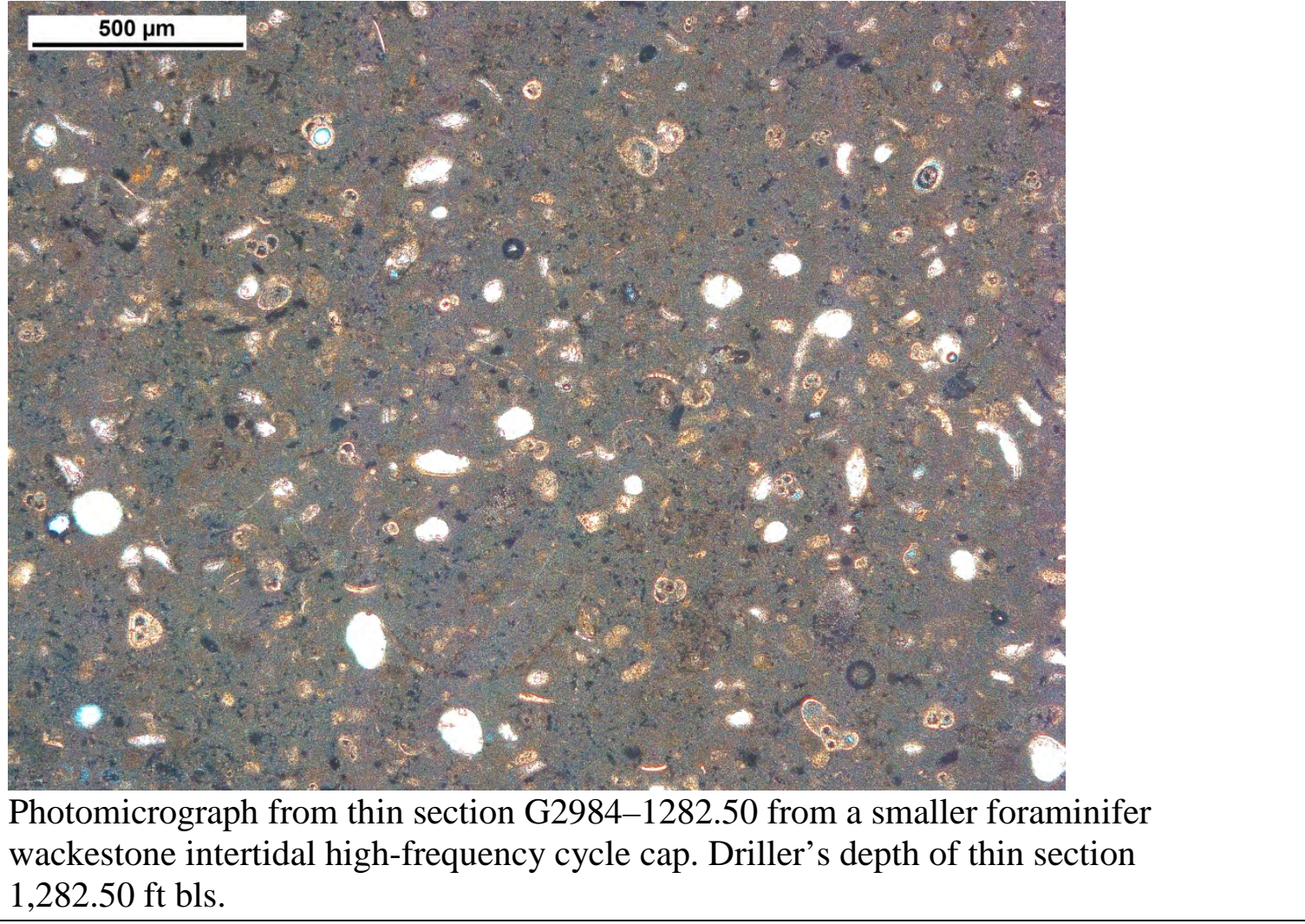 \\
\hline $\begin{array}{l}\text { obi } \\
\text { depth: } \\
\text { 1,284.6- } \\
\text { 1,284.7 ft } \\
\text { bls } \\
\text { Driller's } \\
\text { depth: } \\
\text { 1,283.3- } \\
\text { 1,283.4 ft } \\
\text { bls }\end{array}$ & $\begin{array}{l}\text { Lithofacies: Microbial laminite } \\
\text { Depositional texture: Microbial laminite-packstone } \\
\text { Color: Interlaminated very pale orange } 10 \mathrm{YR} 8 / 2 \text { and pale yellowish brown } 10 \mathrm{YR} 6 / 2 \\
\text { Sedimentary structures: Thinly to thickly laminated, topography-draping laminations } \\
\text { Carbonate grains: Mainly peloids and smaller benthic foraminifera } \\
\text { Porosity and permeability: } 1-5 \text { percent interparticle and intraparticle porosity, and } \\
\text { relatively low permeability } \\
\text { Depositional environment: Low-energy restricted inner platform, tidal flat }\end{array}$ \\
\hline $\begin{array}{l}\text { obi } \\
\text { depth: } \\
\text { 1,284.7- } \\
\text { 1,289.0 ft } \\
\text { bls } \\
\text { Driller's } \\
\text { depth: } \\
\text { 1,283.4- } \\
\text { 1,287.1 ft } \\
\text { bls }\end{array}$ & $\begin{array}{l}\text { Lithofacies: Benthic foraminifer grain-dominated packstone and grainstone } \\
\text { Depositional texture: Smaller and larger benthic foraminifer grain-dominated packstone } \\
\text { and grainstone } \\
\text { Color: Very pale orange } 10 \text { YR } 8 / 2 \\
\text { Sedimentary structures: Burrow mottled, very thickly bedded } \\
\text { Trace fossils: Ophiomorpha? } \\
\text { Ichnofabrics: Ichnofabric index } 5 \\
\text { Ichnofacies: Skolithos? } \\
\text { Carbonate grains: Mainly peloids (probably many micritized unidentified skeletal } \\
\text { grains), smaller benthic foraminifera (including small rotaliids and smaller miliolids), } \\
\text { larger benthic foraminifera (including Fallotella floridana and larger miliolids), minor } \\
\text { Neolaganum dalli, uncommon disarticulated bivalves } \\
\text { Accessory grains: Less than } 1 \text { percent carbonaceous fragments }\end{array}$ \\
\hline
\end{tabular}




\begin{tabular}{|c|c|}
\hline & $\begin{array}{l}\text { Porosity and permeability: 5-25 percent interparticle and intraparticle porosity, 1-3 } \\
\text { percent moldic porosity; 6-28 percent total porosity and moderate permeability } \\
\text { Depositional environment: High-energy inner platform, shallow subtidal }\end{array}$ \\
\hline $\begin{array}{l}\text { obi } \\
\text { depth: } \\
\text { 1,289.0- } \\
\text { 1,293.2 ft } \\
\text { bls } \\
\text { Driller's } \\
\text { depth: } \\
\text { 1,287.1- } \\
\text { 1,291.9 ft } \\
\text { bls }\end{array}$ & $\begin{array}{l}\text { Cycle type: Top type IV cycle } \\
\text { Lithofacies: Benthic foraminifer packstone and grainstone } \\
\text { Depositional texture: Smaller and larger benthic foraminifer grain-dominated packstone } \\
\text { and grainstone with smaller and larger benthic foraminifer mud-dominated packstone in } \\
\text { uppermost } 1 \mathrm{ft} \text { of interval } \\
\text { Color: Very pale orange } 10 \text { YR } 8 / 2 \\
\text { Sedimentary structures: Burrow mottled, very thickly bedded } \\
\text { Trace fossils: Thalassinoides } \\
\text { Ichnofabrics: Ichnofabric index } 5 \\
\text { Ichnofacies: Distal Skolithos? } \\
\text { Carbonate grains: Mainly peloids (probably many micritized unidentified skeletal } \\
\text { grains), smaller benthic foraminifera (including small rotaliids and smaller miliolids), } \\
\text { larger benthic foraminifera (including Fallotella floridana and larger miliolids), minor } \\
\text { Neolaganum dalli, uncommon disarticulated bivalves } \\
\text { Accessory grains: Less than } 1 \text { percent carbonaceous fragments } \\
\text { Porosity and permeability: } 5 \text {-25 percent interparticle and intraparticle porosity, 1-3 } \\
\text { percent moldic porosity; } 6 \text {-28 percent total porosity and moderate permeability } \\
\text { Depositional environment: High-energy inner platform, shallow subtidal } \\
\text { Comments: Minor mud-dominated packstone with a subtidal cycle top and top of a } \\
\text { firmground with an associated underlying Thalassinoides-dominated Glossifungites } \\
\text { ichnofacies at 1,289.0 ft bls (obi depth) and 1,287.1 ft bls (driller's depth) }\end{array}$ \\
\hline $\begin{array}{l}\text { obi } \\
\text { depth: } \\
\text { 1,293.2- } \\
\text { 1,293.35 } \\
\text { ft bls } \\
\text { Driller's } \\
\text { depth: } \\
\text { 1,291.9- } \\
\text { 1,292.05 } \\
\text { ft bls }\end{array}$ & $\begin{array}{l}\text { Cycle type: Top type I cycle } \\
\text { Lithofacies: Microbial laminite } \\
\text { Depositional texture: Microbial laminite-packstone } \\
\text { Color: Pale yellowish brown } 10 \mathrm{YR} 6 / 2 \\
\text { Sedimentary structures: Thinly to thickly laminated, topography-draping laminations } \\
\text { Carbonate grains: Mainly peloids and smaller benthic foraminifera } \\
\text { Porosity and permeability: } 1-15 \text { percent interparticle and intraparticle porosity, and } \\
\text { relatively low permeability } \\
\text { Depositional environment: Low-energy restricted inner platform, tidal flat } \\
\text { Comments: Top of generally fining upward peritidal cycle at } 1,293.2 \mathrm{ft} \text { bls (obi depth) } \\
\text { and } 1,291.9 \mathrm{ft} \text { bls (driller's depth) }\end{array}$ \\
\hline $\begin{array}{l}\text { obi } \\
\text { depth: } \\
1,293.35- \\
1,295.5 \mathrm{ft} \\
\text { bls } \\
\text { Driller's } \\
\text { depth: } \\
1,292.05- \\
1,296.0 \mathrm{ft}\end{array}$ & $\begin{array}{l}\text { Lithofacies: Benthic foraminifer packstone and grainstone } \\
\text { Depositional texture: Smaller and larger benthic foraminifer grain-dominated packstone } \\
\text { and grainstone } \\
\text { Color: Very pale orange 10YR } 8 / 2 \\
\text { Sedimentary structures: Burrow mottled, very thickly bedded } \\
\text { Trace fossils: Small Ophiomorpha } \\
\text { Ichnofabrics: Ichnofabric index } 5 \\
\text { Carbonate grains: Mainly peloids (probably many micritized unidentified skeletal } \\
\text { grains), smaller benthic foraminifera (including small rotaliids and smaller miliolids), } \\
\text { larger benthic foraminifera (including Fallotella floridana and larger miliolids), minor }\end{array}$ \\
\hline
\end{tabular}




\begin{tabular}{|c|c|}
\hline bls & $\begin{array}{l}\text { Neolaganum dalli, uncommon disarticulated bivalves, intraclasts (rip-up clasts of tidal- } \\
\text { flat sediment up to large pebble size) } \\
\text { Accessory grains: Less than } 1 \text { percent carbonaceous fragments } \\
\text { Porosity and permeability: 5-25 percent interparticle and intraparticle porosity, 1-3 } \\
\text { percent moldic porosity; 6-28 percent total porosity and moderate permeability } \\
\text { Depositional environment: High-energy inner platform, shallow subtidal }\end{array}$ \\
\hline $\begin{array}{l}\text { obi } \\
\text { depth: } \\
\text { 1,295.5- } \\
\text { 1,296.6 ft } \\
\text { bls } \\
\text { Driller's } \\
\text { depth: } \\
\text { 1,296.0- } \\
\text { 1,297.3 ft } \\
\text { bls }\end{array}$ & $\begin{array}{l}\text { Lithofacies: Skeletal packstone and grainstone } \\
\text { Depositional texture: Skeletal grain-dominated packstone-grainstone } \\
\text { Color: Very pale orange 10YR 8/2 } \\
\text { Sedimentary structures: Burrow mottled, thickly bedded } \\
\text { Trace fossils: None identified } \\
\text { Ichnofabrics: Ichnofabric index } 5 \\
\text { Carbonate grains: Mainly peloids (probably many micritized unidentified skeletal } \\
\text { grains), pellets, benthic foraminifera, dasycladacean algae, small mollusks (disarticulated } \\
\text { and articulated bivalves and gastropods), intraclasts (rip-up clasts of micrite-dominated } \\
\text { tidal-flat sediment), and small centimeter-scale stick-shaped corals } \\
\text { Porosity and permeability: 5-20 percent interparticle and intraparticle porosity, 1-3 } \\
\text { percent moldic porosity; 6-23 percent total porosity and low permeability } \\
\text { Depositional environment: High-energy inner platform, shallow subtidal }\end{array}$ \\
\hline $\begin{array}{l}\text { obi } \\
\text { depth: } \\
\text { 1,296.6- } \\
\text { 1,296.9 ft } \\
\text { bls } \\
\text { Driller's } \\
\text { depth: } \\
\text { 1,297.3- } \\
\text { 1,297.6 ft } \\
\text { bls }\end{array}$ & $\begin{array}{l}\text { Cycle type: Top type II cycle } \\
\text { Lithofacies: Benthic foraminifer wackestone and packstone } \\
\text { Depositional texture: Smaller benthic foraminifer wackestone and mud-dominated } \\
\text { packstone } \\
\text { Color: Very light gray N8 } \\
\text { Sedimentary structures: Thickly laminated, vertical desiccation cracks, fenestral fabric } \\
\text { Trace fossils: Semivertical rhizoliths (some bifurcation of rhizolith tubes foraminifera } \\
\text { observed) } \\
\text { Ichnofabrics: Ichnofabric index 1-2? } \\
\text { Ichnofacies: Psilonichnus } \\
\text { Carbonate grains: Mainly smaller benthic foraminifera (including small rotaliids and a } \\
\text { few small miliolids and biserial foraminifera) and peloids, minor ostracods, intraclasts, } \\
\text { uncommon larger miliolids. Foraminifera observed in thin section G2984-1297.35 } \\
\text { include smaller benthic foraminifera } \\
\text { Porosity and permeability: } 1-4 \text { percent interparticle and intraparticle porosity, 1-3 } \\
\text { percent root-mold porosity, } 1 \text { percent fenestral fabric; } 2-7 \text { percent total porosity and } \\
\text { relatively low permeability } \\
\text { Depositional environment: Low-energy restricted inner platform, intertidal to } \\
\text { supratidal } \\
\text { Comments: Top of generally fining upward peritidal cycle at } 1,296.6 \text { ft bls (obi depth) } \\
\text { and } 1,297.3 \text { ft bls (driller’s depth) } \\
\text { Thin section: G2984-1297.35 }\end{array}$ \\
\hline
\end{tabular}




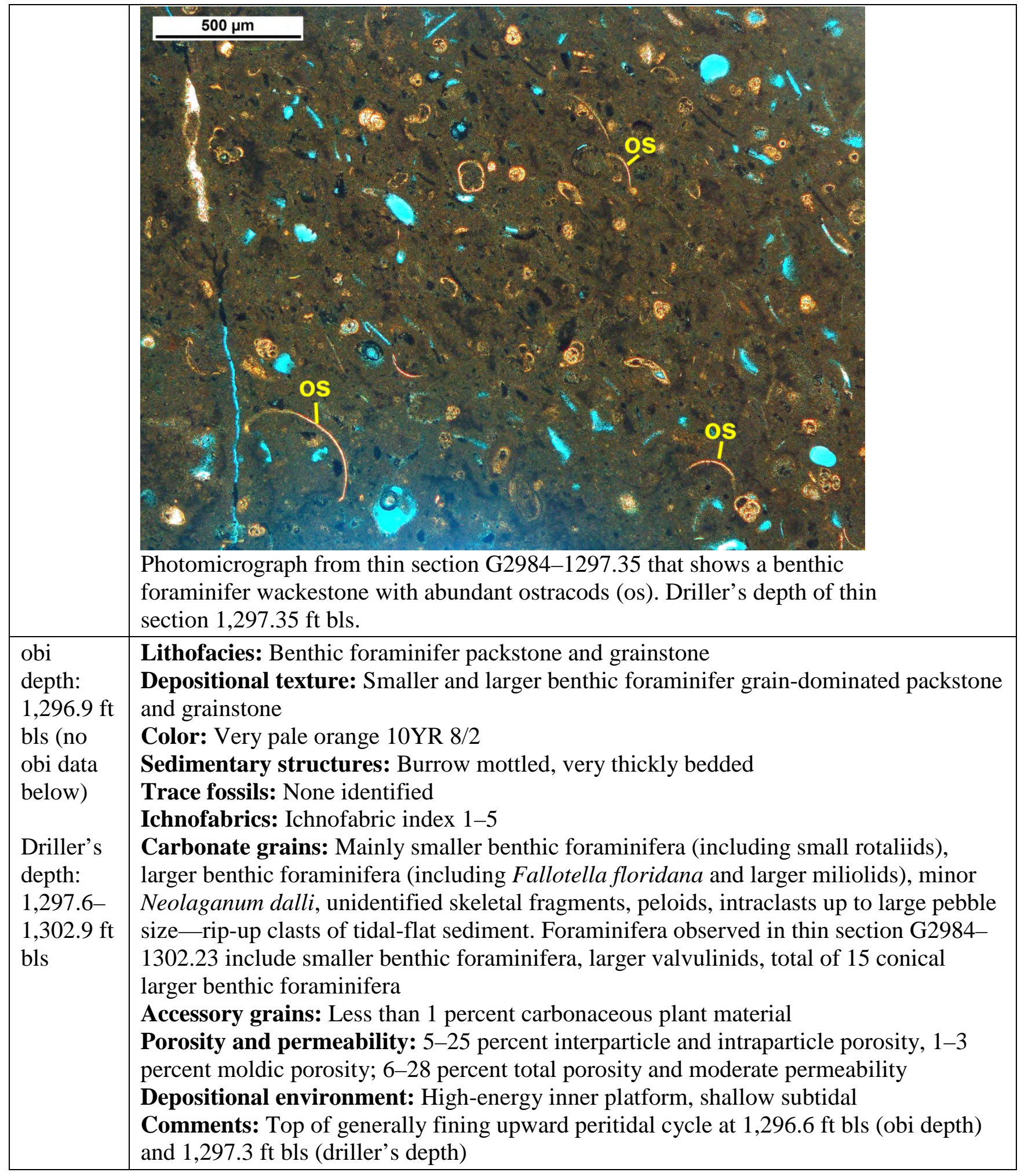




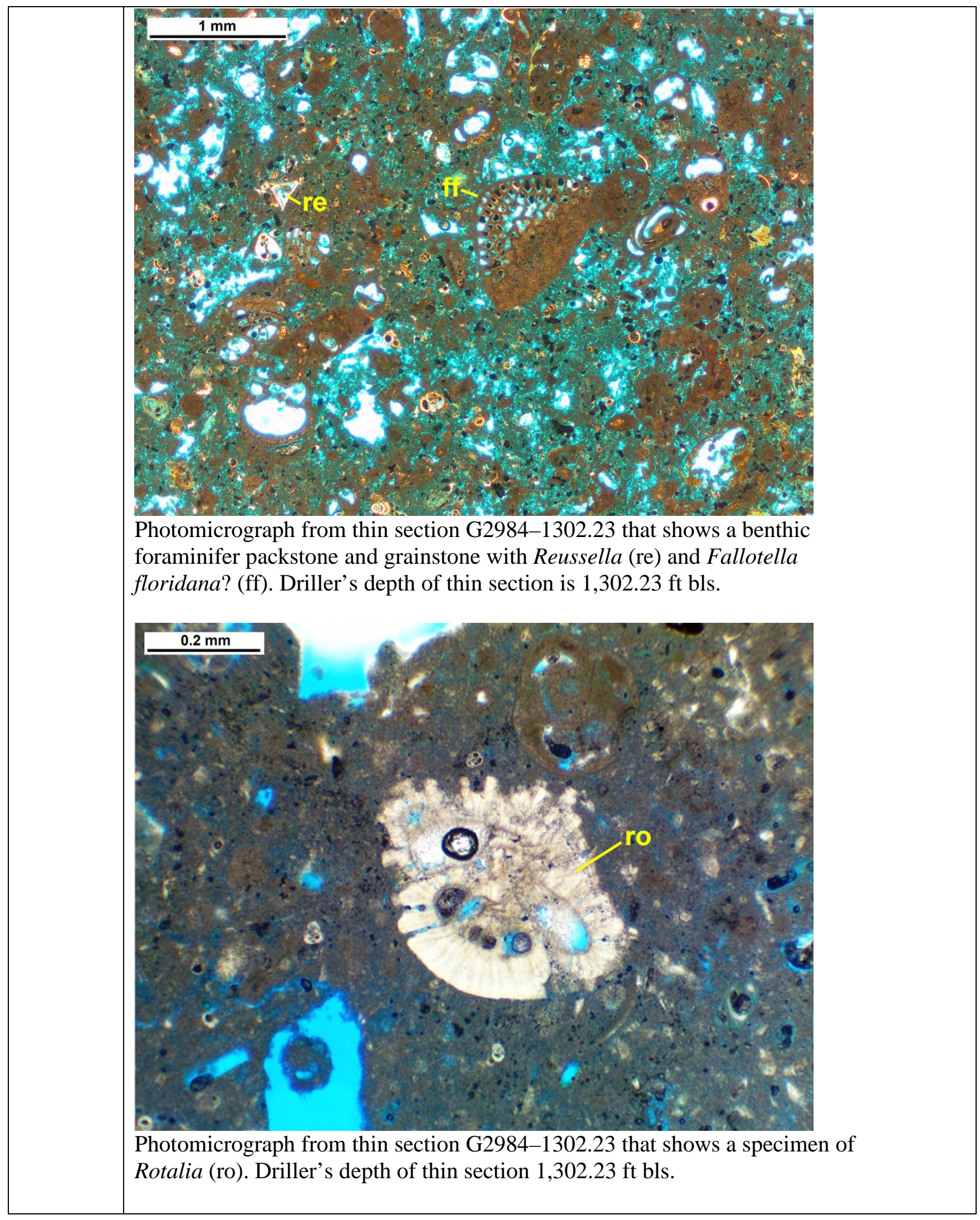




\begin{tabular}{|c|c|}
\hline & $\begin{array}{l}\text { Photomicrograph from thin section G2984-1302.23 that shows a specimen of } \\
\text { Valvulina avon-parkensis (vp). Driller's depth of thin section } 1,302.23 \mathrm{ft} \text { bls. }\end{array}$ \\
\hline $\begin{array}{l}\text { Driller's } \\
\text { depth: } \\
1,302.9- \\
1,303.2 \mathrm{ft} \\
\text { bls }\end{array}$ & $\begin{array}{l}\text { Lithofacies: Rip-up clast floatstone } \\
\text { Depositional texture: Intraclast floatstone with matrix of smaller and larger benthic } \\
\text { foraminifer grain-dominated packstone and grainstone } \\
\text { Color: Very pale orange 10YR 8/2 } \\
\text { Sedimentary structures: Burrow mottled, thinly bedded } \\
\text { Trace fossils: None identified } \\
\text { Ichnofabrics: Ichnofabric index } 5 \\
\text { Carbonate grains: Mainly peloids (probably many micritized unidentified skeletal } \\
\text { grains), smaller benthic foraminifera (including small rotaliids and smaller miliolids), } \\
\text { larger benthic foraminifera (including Fallotella floridana, Coskinolina floridana, and } \\
\text { larger miliolids), minor rip-up intraclasts up to medium pebble size (rip-ups of } \\
\text { underlying benthic foraminifer wackestone-tidal-flat sediment). Foraminifera observed } \\
\text { in thin section G2984-1303.13 include smaller benthic foraminifera, Coskinolina } \\
\text { floridana, total of seven conical larger benthic foraminifera } \\
\text { Porosity and permeability: } 5-15 \text { percent interparticle and intraparticle porosity, 1-3 } \\
\text { percent moldic porosity; } 6-18 \text { percent total porosity and low permeability } \\
\text { Depositional environment: High-energy event, marine subtidal. Marine erosion of } \\
\text { tidal-flat sediments } \\
\text { Comment: Marine erosion of underlying tidal-flat substrate } \\
\text { Thin section: G2984-1303.13 }\end{array}$ \\
\hline $\begin{array}{l}\text { Driller's } \\
\text { depth: } \\
1,303.2- \\
1,304.7 \mathrm{ft} \\
\text { bls }\end{array}$ & $\begin{array}{l}\text { Cycle type: Top type II cycle } \\
\text { Lithofacies: Benthic foraminifer wackestone and packstone } \\
\text { Depositional texture: Smaller benthic foraminifer wackestone } \\
\text { Color: Very light gray N8 to light gray N7 } \\
\text { Sedimentary structures: Thinly bedded to medium bedded. Uncommon very thinly (1- }\end{array}$ \\
\hline
\end{tabular}


$2 \mathrm{~mm}$ thick) laminated organic-rich algal mats approximately in middle of interval

Trace fossils: Semivertical to minor semihorizontal rhizoliths (some bifurcation of rhizolith tubes)

Ichnofabrics: Ichnofabric index 1-2

Ichnofacies: Psilonichnus

Carbonate grains: Mainly smaller benthic foraminifera (including small rotaliids and a few small miliolids and biserial foraminifera) and peloids, minor ostracods, intraclasts, uncommon larger miliolids and conical larger benthic foraminifera. Foraminifera observed in thin section G2984-1303.38 include smaller benthic foraminifera. Foraminifera observed in thin section G2984-1303.65 include smaller benthic foraminifera. Foraminifera observed in thin section G2984-1304.00 include Fallotella floridana, smaller benthic foraminifera, total of three conical larger benthic foraminifera. Foraminifera observed in thin section G2984-1304.42 include Dendritina sp., larger valvulinids, Coskinolina floridana, total of 12 conical larger benthic foraminifera Porosity and permeability: 1-5 percent interparticle and intraparticle porosity, 1-3 percent root-mold porosity, 1-3 percent irregular vugs; 3-11 percent total porosity and relatively low permeability

Depositional environment: Low-energy restricted inner platform, tidal flat Comments: Top of generally fining upward peritidal cycle at 1,303.0 ft bls (driller's depth). Conical benthic foraminifera are concentrated at the base of the unit and may be mixed upward by bioturbation from conical benthic foraminifera-rich grainstones underlying the unit

Thin section: G2984-1303.38, G2984-1303.65, G2984-1304.00, G2984-1304.42

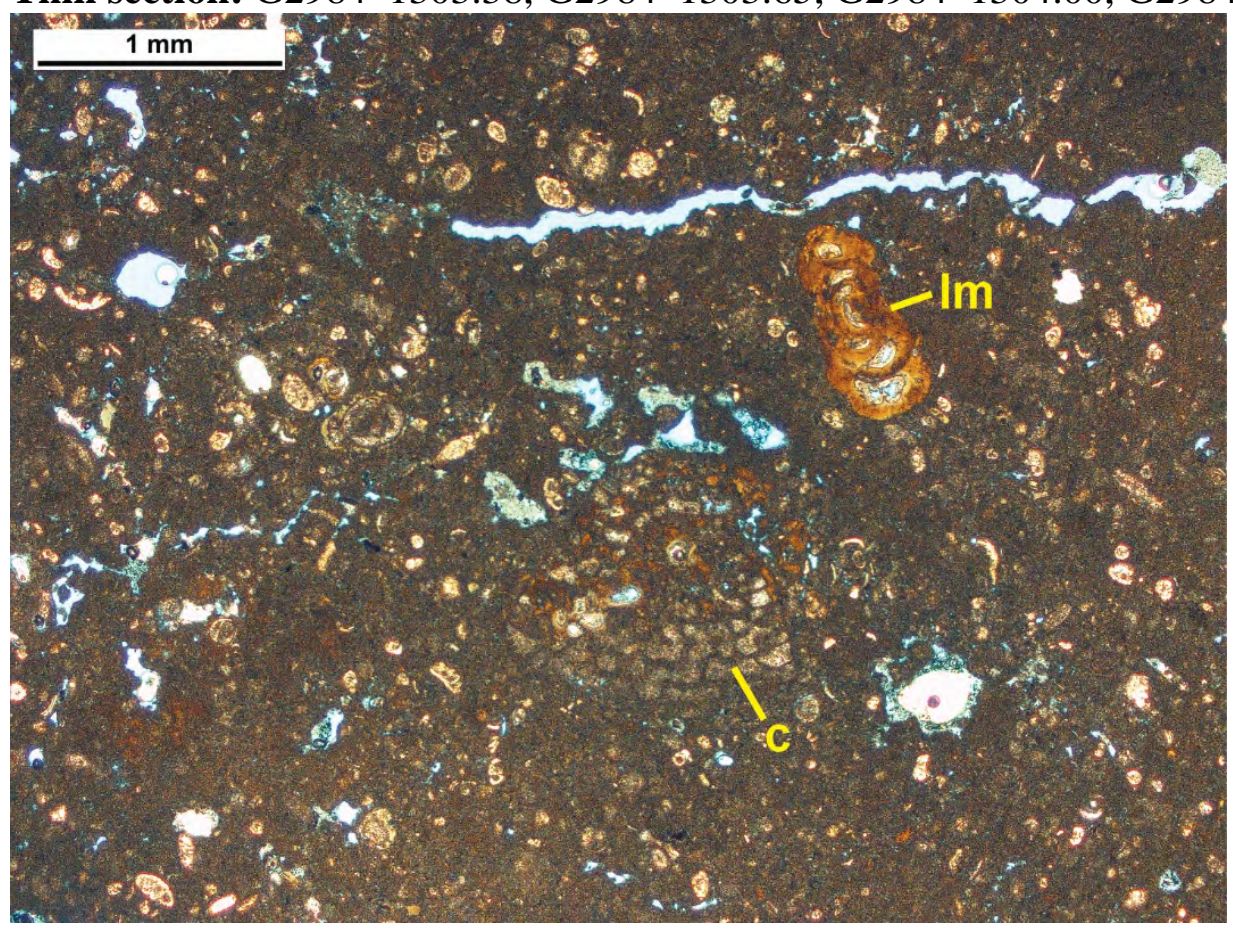

Photomicrograph from thin section G2984-1304.42 that shows a smaller benthic foraminifer wackestone that contains a conical larger benthic foraminifer (c) and larger miliolid (lm). Driller's depth of thin section is $1,304.42 \mathrm{ft}$ bls. 


\begin{tabular}{|c|c|}
\hline $\begin{array}{l}\text { Driller's } \\
\text { depth: } \\
1,304.7- \\
1,307.0 \mathrm{ft} \\
\text { bls }\end{array}$ & $\begin{array}{l}\text { Lithofacies: Benthic foraminifer packstone and grainstone } \\
\text { Depositional texture: Smaller and larger benthic foraminifer grain-dominated packstone } \\
\text { and grainstone } \\
\text { Color: Very pale orange 10YR 8/2 } \\
\text { Sedimentary structures: Burrow mottled, thickly bedded } \\
\text { Trace fossils: None identified } \\
\text { Ichnofabrics: Ichnofabric index } 5 \\
\text { Carbonate grains: Mainly smaller benthic foraminifera (including small rotaliids), } \\
\text { larger benthic foraminifera (including Fallotella floridana, Coskinolina floridana, and } \\
\text { larger miliolids), minor Neolaganum dalli, unidentified skeletal fragments, peloids, } \\
\text { intraclasts up to small pebble size, ostracods, thin disarticulated bivalves, one stick- } \\
\text { shaped coral, Microcodium. Foraminifera observed in thin section G2984-1305.16 } \\
\text { include Dendritina sp. Foraminifera observed in thin section G2984-1306.10 include } \\
\text { smaller benthic foraminifera, larger valvulinids, Coskinolina floridana, Arenagula sp., } \\
\text { total of } 12 \text { conical larger benthic foraminifera } \\
\text { Porosity and permeability: } 5 \text {-25 percent interparticle and intraparticle porosity, 1-3 } \\
\text { percent moldic porosity; 6-28 percent total porosity and moderate permeability } \\
\text { Depositional environment: High-energy inner platform, shallow subtidal } \\
\text { Thin section: G2984-1305.16, G2984-1306.10 } \\
1 \text { mm }\end{array}$ \\
\hline $\begin{array}{l}\text { Driller's } \\
\text { depth: } \\
1,307.0- \\
1,307.3 \mathrm{ft}\end{array}$ & $\begin{array}{l}\text { Lithofacies: Rip-up clast floatstone } \\
\text { Depositional texture: Intraclast floatstone with matrix of smaller and larger benthic } \\
\text { foraminifer grain-dominated packstone and grainstone } \\
\text { Color: Very pale orange 10YR } 8 / 2\end{array}$ \\
\hline
\end{tabular}




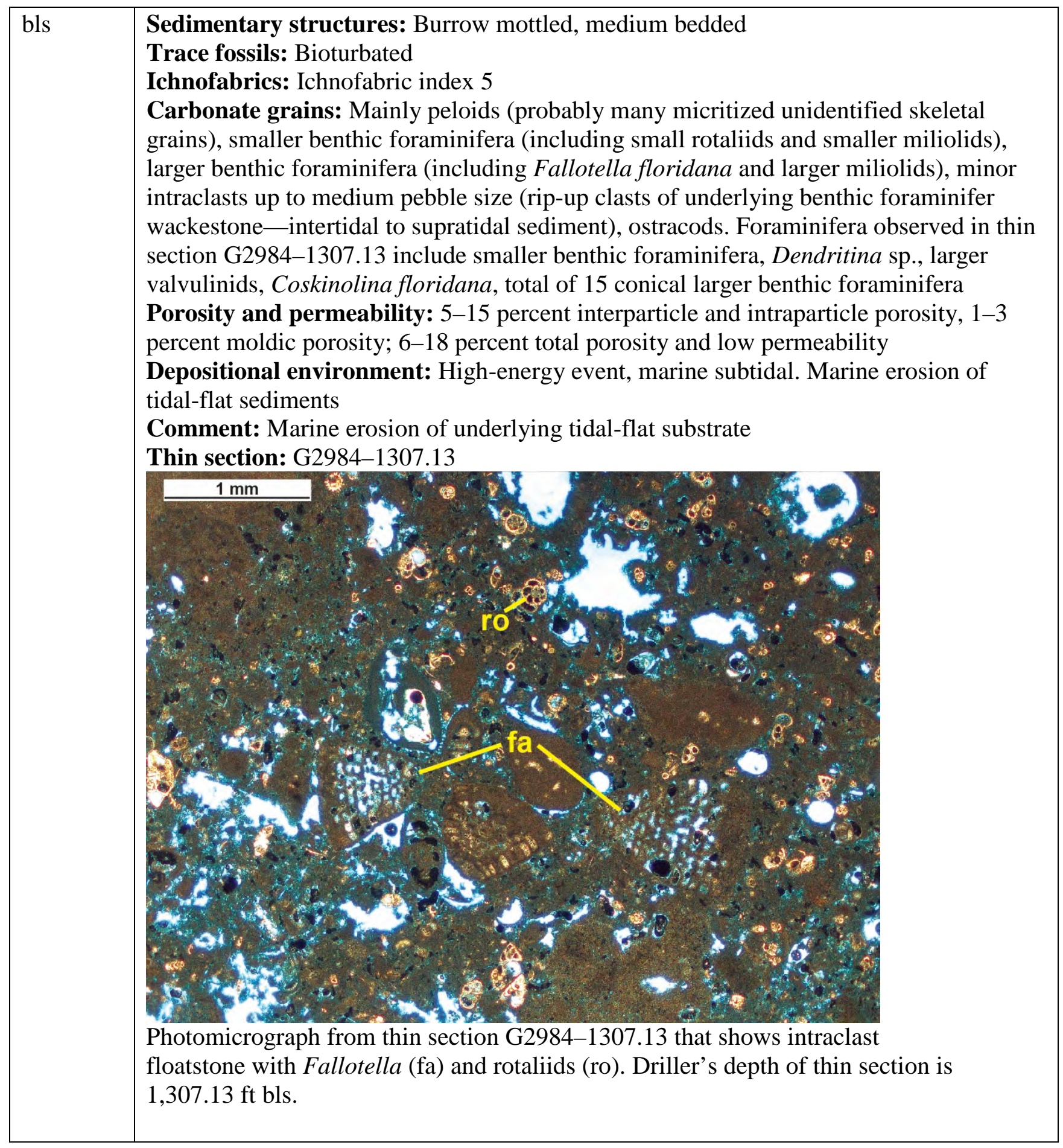




\begin{tabular}{|c|c|}
\hline & 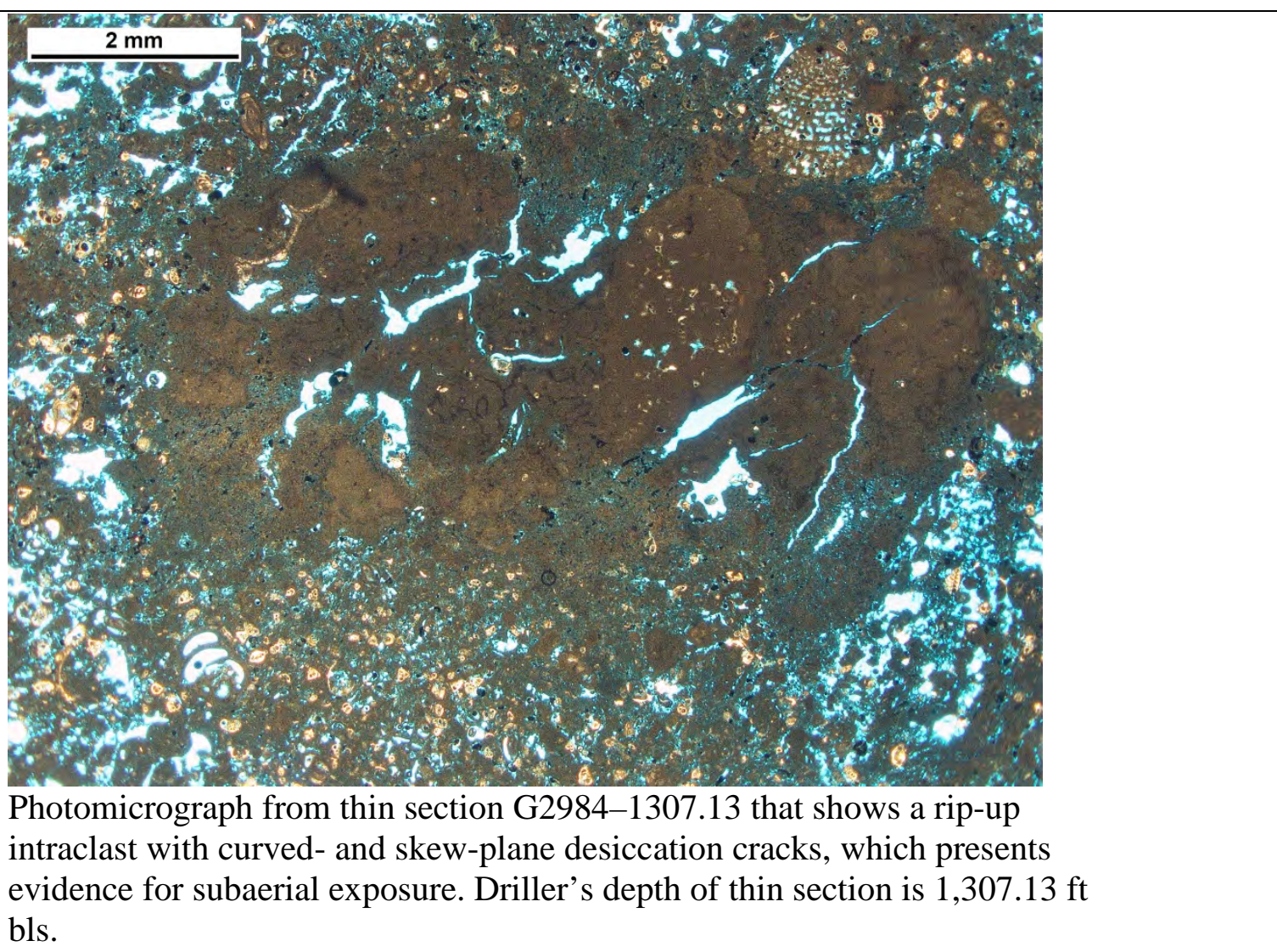 \\
\hline $\begin{array}{l}\text { Driller's } \\
\text { depth: } \\
\text { 1,307.3- } \\
\text { 1,307.7 ft } \\
\text { bls (total } \\
\text { depth) }\end{array}$ & $\begin{array}{l}\text { Cycle type: Top-type II cycle? } \\
\text { Lithofacies: Benthic foraminifer wackestone and packstone } \\
\text { Depositional texture: Smaller benthic foraminifer wackestone } \\
\text { Color: Very light gray N8 } \\
\text { Sedimentary structures: Thinly bedded, fenestrae, desiccation cracks (mainly craze } \\
\text { plane and skew plane) } \\
\text { Trace fossils: Rhizoliths } \\
\text { Ichnofabrics: Ichnofabric index uncertain } \\
\text { Ichnofacies: Psilonichnus } \\
\text { Carbonate grains: Mainly smaller benthic foraminifera (including small rotaliids and a } \\
\text { few small miliolids and biserial foraminifera) and peloids, minor ostracods, intraclasts, } \\
\text { uncommon larger miliolids, and other larger benthic foraminifera (larger miliolids, } \\
\text { Fallotella floridana). Foraminifera observed in thin section G2984-1307.50 include total } \\
\text { of two conical larger benthic foraminifera } \\
\text { Porosity and permeability: } 1 \text {-5 percent interparticle and intraparticle porosity, } 1-5 \\
\text { percent fenestrae porosity, } 1 \text {-3 percent root-mold porosity, 1-2 percent desiccation crack } \\
\text { porosity; } 4 \text {-15 percent total porosity and relatively low permeability } \\
\text { Depositional environment: Low-energy restricted inner platform, intertidal to } \\
\text { supratidal } \\
\text { Comments: Top of generally fining upward peritidal cycle at 1,307.3 ft bls (driller's } \\
\text { depth). Conical benthic foraminifera are concentrated at the top of the unit and may be } \\
\text { mixed downward by bioturbation from conical benthic foraminifera-rich grainstones } \\
\text { overlying the unit }\end{array}$ \\
\hline
\end{tabular}




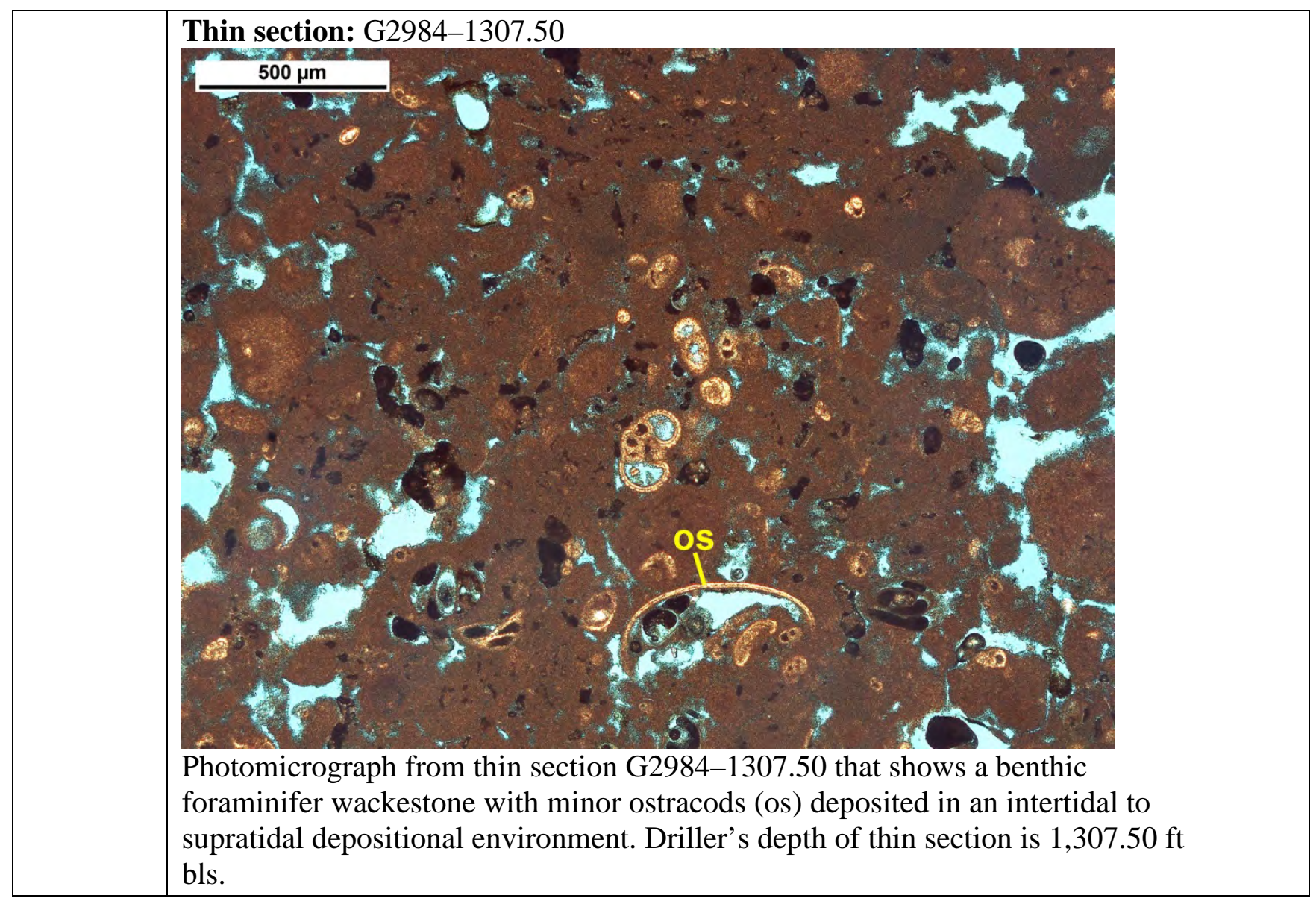




\section{Lithofacies Description and Sequence Stratigraphy of Continuously Drilled Samples from the Arcadia Formation at U.S. Geological Survey G-2984 Test Corehole}

\begin{tabular}{|c|c|}
\hline $\begin{array}{l}\text { Depth } \\
\text { interval } \\
\text { (using } \\
\text { obi log } \\
\text { depth } \\
\text { and } \\
\text { driller's } \\
\text { depth, in } \\
\text { feet bls) }\end{array}$ & $\begin{array}{l}\text { Estimates of permeability: Based on comparison of Arcadia Formation to lithofacies and } \\
\text { pore types to } 276 \text { Pliocene and Pleistocene eogenetic carbonate rock specimens with } \\
\text { similar lithofacies and pore types and their air-permeability measurements (Cunningham } \\
\text { and others, 2006) and lattice Boltzmann permeability calculations of both Pleistocene and } \\
\text { Cretaceous carbonate rocks (Cunningham and others, 2009, 2012; Cunningham and } \\
\text { Sukop, 2011, 2012; Sukop and others, 2013; Sukop and Cunningham, 2014) } \\
\text { Colors: Colors based on comparison to Munsell rock color chart (Geological Society of } \\
\text { America, 1991) } \\
\text { Ichnofabric: Index based on Droser and Bottjer (1986, 1989) } \\
\text { Top of Arcadia Formation: } 596.25 \mathrm{ft} \text { bls (obi depth) and 592.25 ft bls (driller's depth) } \\
\text { Base of Arcadia Formation: } 1,067.60 \mathrm{ft} \text { bls (obi depth) and 1,067.60 ft bls (driller's } \\
\text { depth) }\end{array}$ \\
\hline $\begin{array}{l}\text { obi } \\
\text { depth: } \\
596.25- \\
597 \mathrm{ft} \\
\text { bls } \\
\text { Driller's } \\
\text { depth: } \\
592.25- \\
593 \mathrm{ft} \\
\text { bls }\end{array}$ & $\begin{array}{l}\text { Lithofacies: Phosphorite } \\
\text { Depositional texture: Phosphorite } \\
\text { Color: Black N1 and pale yellowish brown } 10 \mathrm{YR} 6 / 2 \\
\text { Sedimentary structures: Burrow mottled } \\
\text { Ichnofabrics: Ichnofabric index } 5 \\
\text { Ichnofacies: Glossifungites } \\
\text { Accessory grains: Foraminifera observed in thin section G2984-592.82 include } \\
\text { Globigerinoides gr. trilobus, bolivinids, total of } 10 \text { planktic foraminifera, total of } 3 \\
\text { smaller benthic foraminifera } \\
\text { Porosity and permeability: } 3 \text { percent interparticle porosity, } 1 \text { percent intraparticle } \\
\text { porosity, } 3 \text { percent moldic porosity; } 7 \text { percent total porosity and low permeability } \\
\text { Depositional environment: Outer ramp } \\
\text { Comments: Thalassinoides-dominated Glossifungites extends downward from top of } \\
\text { cycle } 3.5 \mathrm{ft} \text { and contains fill dominated by globular planktic foraminifera. Uppermost part } \\
\text { of cycle is a firmground that was later transformed to a hardground by phosphatization. } \\
\text { Top of depositional sequence (fig. } 2 \text { ) at } 592.25 \mathrm{ft} \text { (driller’s depth) } \\
\text { Thin section: G2984-592.82 }\end{array}$ \\
\hline $\begin{array}{l}\text { obi } \\
\text { depth: } \\
\text { 597-605 } \\
\text { ft bls } \\
\text { Driller's } \\
\text { depth: } \\
\text { 593-605 } \\
\text { ft bls }\end{array}$ & $\begin{array}{l}\text { Lithofacies: Foraminifer wackestone to packstone } \\
\text { Depositional texture: Smaller benthic and globular planktic foraminifer wackestone to } \\
\text { mud-dominated packstone } \\
\text { Color: Yellowish gray 5Y 8/1 } \\
\text { Sedimentary structures: Burrow mottled } \\
\text { Trace fossils: Thalassinoides, Rhizocorallium? } \\
\text { Ichnofabrics: Ichnofabric index } 5 \\
\text { Ichnofacies: Cruziana and Thalassinoides-dominated Glossifungites at top of unit } \\
\text { Carbonate grains: Mainly silt to very fine sand-sized angular skeletal fragments, } \\
\text { globular planktic foraminifera, smaller benthic foraminifera (including rotaliids), and } \\
\text { minor ostracods. Foraminifera observed in thin section G2984-593.00 include Orbulina }\end{array}$ \\
\hline
\end{tabular}




\begin{tabular}{|c|c|}
\hline & $\begin{array}{l}\text { suturalis?, Globigerinoides gr. trilobus, rotaliids (rare), planktic foraminifera (dominant), } \\
\text { smaller benthic foraminifera with bolivinids (dominant). Foraminifera observed in thin } \\
\text { section G2984-599.13 include Globigerinoides gr. trilobus, unidentified planktic } \\
\text { foraminifera, bolivinids, planktic foraminifera, smaller benthic foraminifera. Mud to } \\
\text { coarse sand-sized carbonate grains } \\
\text { Accessory grains: } 1-5 \text { percent silt to very fine sand-sized, angular, well sorted quartz } \\
\text { grains; } 1-2 \text { percent silt to very fine to coarse sand-sized phosphorite grains; } 1-3 \text { percent } \\
\text { very fine sand-sized dolomite rhombs } \\
\text { Porosity and permeability: } 4 \text { percent interparticle porosity, } 1 \text { percent intraparticle } \\
\text { porosity, } 3 \text { percent moldic porosity; } 8 \text { percent total porosity and low permeability } \\
\text { Depositional environment: Outer ramp } \\
\text { Comments: Thalassinoides-dominated Glossifungites extends downward } 3.5 \mathrm{ft} \text { from top } \\
\text { of cycle at 592.25 ft bls (driller's depth) and contains fill dominated by globular planktic } \\
\text { foraminifera. } \\
\text { Thin section: G2984-593.00, G2984-599.13 }\end{array}$ \\
\hline $\begin{array}{l}\text { obi } \\
\text { depth: } \\
605- \\
605.5 \mathrm{ft} \\
\text { bls } \\
\text { Driller's } \\
\text { depth: } \\
605- \\
605.5 \mathrm{ft} \\
\text { bls }\end{array}$ & $\begin{array}{l}\text { Lithofacies: Foraminifer packstone } \\
\text { Depositional texture: Smaller benthic and globular planktic foraminifer mud-dominated } \\
\text { packstone } \\
\text { Color: Yellowish gray } 5 Y \text { Y } 1 \\
\text { Sedimentary structures: Burrow mottled } \\
\text { Trace fossils: Thalassinoides } \\
\text { Ichnofabrics: Ichnofabric index } 5 \\
\text { Ichnofacies: Cruziana? } \\
\text { Carbonate grains: Mainly silt to very fine sand-sized angular skeletal fragments, smaller } \\
\text { benthic foraminifera, globular planktic foraminifera, and minor ostracods, bivalve } \\
\text { fragments. Foraminifera observed in thin section G2984-605.25 include Praeorbulina } \\
\text { sicanus?, Globoquadrina sp., Globigerinoides gr. trilobus, unidentified planktic } \\
\text { foraminifera, bolivinids, lenticulinids, planktic foraminifera (dominant), smaller benthic } \\
\text { foraminifera } \\
\text { Accessory grains: } 1-3 \text { percent silt to very fine sand-sized, angular, well sorted quartz } \\
\text { grains; } 5-7 \text { percent silt to very fine to small pebble-sized phosphorite grains decreasing } \\
\text { upward } \\
\text { Porosity and permeability: } 4 \text { percent interparticle porosity, } 1 \text { percent intraparticle } \\
\text { porosity; } 5 \text { percent total porosity and low permeability } \\
\text { Depositional environment: Outer ramp } \\
\text { Comments: Base of fining upward cycle at } 605.5 \text { ft bls (driller’s depth) that fines upward } \\
\text { to top of cycle at } 592.25 \text { ft bls (driller's depth) } \\
\text { Thin section: G2984-605.25 }\end{array}$ \\
\hline $\begin{array}{l}\text { obi } \\
\text { depth: } \\
605.5- \\
616 \mathrm{ft} \\
\text { bls }\end{array}$ & $\begin{array}{l}\text { Lithofacies: Foraminifer wackestone to packstone } \\
\text { Depositional texture: Smaller benthic and globular planktic foraminifer wackestone to } \\
\text { mud-dominated packstone } \\
\text { Color: Yellowish gray } 5 Y \text { 8/1 } \\
\text { Sedimentary structures: Burrow mottled } \\
\text { Trace fossils: Thalassinoides, Rhizocorallium? } \\
\text { Ichnofabrics: Ichnofabric index } 5 \\
\text { Ichnofacies: Cruziana with a Glossifungites ichnofacies capping the cycle }\end{array}$ \\
\hline
\end{tabular}




\begin{tabular}{|c|c|}
\hline $\begin{array}{l}605.5- \\
616 \mathrm{ft} \\
\text { bls }\end{array}$ & $\begin{array}{l}\text { Carbonate grains: Mainly silt to very fine sand-sized angular skeletal fragments, smaller } \\
\text { benthic foraminifera, globular planktic foraminifera, and minor ostracods, bivalve } \\
\text { fragments. Foraminifera observed in thin section G2984-606.76 include Praeorbulina } \\
\text { glomerosa s.l., unidentified planktic foraminifera, bolivinids (dominant), planktic } \\
\text { foraminifera, smaller benthic foraminifera (dominant). Foraminifera observed in thin } \\
\text { section G2984-611.90 include Praeorbulina glomerosa s.l., unidentified planktic } \\
\text { foraminifera, bolivinids (dominant), lenticulinids, planktic foraminifera, smaller benthic } \\
\text { foraminifera (dominant) } \\
\text { Accessory grains: } 1-7 \text { percent silt to very fine sand-sized, angular, well sorted quartz } \\
\text { grains; } 5 \text {-7 percent silt to very fine to coarse sand-sized phosphorite grains over lower } 0.5 \\
\text { ft of cycle and overlying part of cycle contains } 1 \text { percent silt to fine sand-sized black } \\
\text { phosphorite grains; } 1-3 \text { percent very fine sand-sized dolomite rhombs } \\
\text { XRD mineralogy: At } 606.76 \text { ft bls (driller's depth), whole rock mineralogy in weight } \\
\text { percentage (quartz } 4.8 \text { percent, calcite } 64.6 \text { percent, dolomite and [Iron (Fe),Calcium } \\
\text { (Ca)]-dolomite } 16.9 \text { percent, total clay minerals } 13.7 \text { percent); clay mineralogy in weight } \\
\text { percentage (illite/smectite* } 3.8 \text { percent, palygorskite } 9.9 \text { percent). *Mixed-layer } \\
\text { illite/smectite that contains } 70-80 \text { percent smectite layers } \\
\text { XRD mineralogy: At } 611.90 \mathrm{ft} \text { bls (driller's depth), whole rock mineralogy in weight } \\
\text { percentage (quartz } 9.6 \text { percent, k-feldspar } 1.0 \text { percent, plagioclase } 1.7 \text { percent, calcite } 57.2 \\
\text { percent, dolomite and [Fe,Ca]-dolomite } 16.7 \text { percent, total clay minerals } 13.8 \text { percent); } \\
\text { clay mineralogy in weight percentage (illite/smectite* } 4.5 \text { percent, palygorskite } 9.3 \\
\text { percent). *Mixed-layer illite/smectite that contains } 70-80 \text { percent smectite layers } \\
\text { Porosity and permeability: } 2 \text { percent interparticle porosity, } 1 \text { percent intraparticle } \\
\text { porosity; } 3 \text { percent total porosity and low permeability } \\
\text { Depositional environment: Outer ramp } \\
\text { Comments: Base of fining upward cycle at } 616 \mathrm{ft} \text { (driller's depth) that fines upward to } \\
\text { top of cycle at } 605.6 \mathrm{ft} \text { (driller's depth) and } 1 \mathrm{ft} \text { thick Thalassinoides-dominated } \\
\text { Glossifungites ichnofacies } \\
\text { Thin section: G606.76, G2984-611.90 }\end{array}$ \\
\hline $\begin{array}{l}\text { obi } \\
\text { depth: } \\
616- \\
620.7 \mathrm{ft} \\
\text { bls } \\
\\
\text { Driller's } \\
\text { depth: } \\
616- \\
620.8 \mathrm{ft} \\
\text { bls }\end{array}$ & $\begin{array}{l}\text { Lithofacies: Foraminifer mudstone to packstone } \\
\text { Depositional texture: Smaller benthic and globular planktic foraminifer mudstone to } \\
\text { mud-dominated packstone } \\
\text { Color: Yellowish gray 5Y 7/2 } \\
\text { Sedimentary structures: Burrow mottled } \\
\text { Trace fossils: Thalassinoides, Rosselia? } \\
\text { Ichnofabrics: Ichnofabric index } 5 \\
\text { Ichnofacies: Cruziana with a Glossifungites ichnofacies capping the cycle } \\
\text { Carbonate grains: Mainly silt to very fine sand-sized angular skeletal fragments, smaller } \\
\text { benthic foraminifera, globular planktic foraminifera, and minor ostracods } \\
\text { Accessory grains: } 1 \text { percent silt to very fine sand-sized, angular, well sorted quartz } \\
\text { grains; } 1-2 \text { percent silt to very fine to medium sand-sized black phosphorite grains } \\
\text { XRD mineralogy: At } 619.50 \text { ft bls (driller's depth), whole rock mineralogy in weight } \\
\text { percentage (quartz } 9.1 \text { percent, calcite } 15.0 \text { percent, dolomite and [Fe,Ca]-dolomite } 29.7 \\
\text { percent, total clay minerals } 46.2 \text { percent); clay mineralogy in weight percentage } \\
\text { (illite/smectite* } 20.6 \text { percent, palygorskite } 25.2 \text { percent, kaolinite } 0.4 \text { percent). *Mixed- } \\
\text { layer illite/smectite that contains } 70-80 \text { percent smectite layers }\end{array}$ \\
\hline
\end{tabular}




\begin{tabular}{|c|c|}
\hline & $\begin{array}{l}\text { Porosity and permeability: } 2 \text { percent interparticle porosity, } 1 \text { percent intraparticle } \\
\text { porosity; } 3 \text { percent total porosity and low permeability } \\
\text { Depositional environment: Outer ramp } \\
\text { Comments: Top of fining upward cycle at } 616 \mathrm{ft} \text { bls (driller's depth) that is capped by a } 1 \\
\mathrm{ft} \text { thick Thalassinoides-dominated Glossifungites ichnofacies. Upper surface of cycle is a } \\
\text { firmground }\end{array}$ \\
\hline $\begin{array}{l}\text { obi } \\
\text { depth: } \\
620.7- \\
621.5 \mathrm{ft} \\
\text { bls } \\
\text { Driller's } \\
\text { depth: } \\
620.8- \\
621.6 \mathrm{ft} \\
\text { bls }\end{array}$ & $\begin{array}{l}\text { Lithofacies: Foraminifer wackestone and packstone } \\
\text { Depositional texture: Smaller benthic and globular planktic foraminifer wackestone and } \\
\text { mud-dominated packstone } \\
\text { Color: Yellowish gray } 5 Y \text { Y } 7 / 2 \\
\text { Sedimentary structures: Burrow mottled } \\
\text { Trace fossils: None identified } \\
\text { Ichnofabrics: Ichnofabric index } 5 \\
\text { Ichnofacies: Cruziana? } \\
\text { Carbonate grains: Mainly silt to very fine sand-sized angular skeletal fragments, smaller } \\
\text { benthic foraminifera, globular planktic foraminifera, and minor ostracods, echinoid } \\
\text { spines, bivalve fragments } \\
\text { Accessory grains: } 1 \text { percent silt to very fine sand-sized, angular, well sorted quartz } \\
\text { grains; } 5-7 \text { percent silt to very fine to coarse sand-sized black phosphorite grains } \\
\text { Porosity and permeability: } 2 \text { percent interparticle porosity, } 1 \text { percent intraparticle } \\
\text { porosity; } 3 \text { percent total porosity and low permeability } \\
\text { Depositional environment: Outer ramp } \\
\text { Comments: Base of fining upward cycle at } 621.6 \mathrm{ft} \text { bls (driller’s depth) that fines upward } \\
\text { to top of cycle at } 616 \mathrm{ft} \text { obi depth and capped by a } 1 \mathrm{ft} \text { thick Thalassinoides-dominated } \\
\text { Glossifungites ichnofacies. Upper surface of cycle is a firmground }\end{array}$ \\
\hline $\begin{array}{l}\text { obi } \\
\text { depth: } \\
621.5- \\
622.0 \mathrm{ft} \\
\text { bls } \\
\text { Driller's } \\
\text { depth: } \\
621.6- \\
622.1 \mathrm{ft} \\
\text { bls }\end{array}$ & $\begin{array}{l}\text { Lithofacies: Coral floatstone } \\
\text { Depositional texture: Coral floatstone with a skeletal and globular planktic foraminifer } \\
\text { wackestone matrix } \\
\text { Color: Yellowish gray 5Y 8/1 } \\
\text { Trace fossils: Thalassinoides } \\
\text { Ichnofabrics: Ichnofabric index } 5 \\
\text { Ichnofacies: Glossifungites ichnofacies capping the cycle } \\
\text { Carbonate grains: Mainly silt to very fine sand-sized angular skeletal fragments, } \\
\text { globular planktic foraminifera, small (3-mm diameter) solitary corals, smaller benthic } \\
\text { foraminifera (including rotaliids), and echinoid spines. Foraminifera observed in thin } \\
\text { section G2984-621.75 include Orbulina suturalis?, Praeorbulina glomerosa s.l., } \\
\text { unidentified planktic foraminifera, bolivinids, lenticulinids, rotaliids, planktic foraminifera } \\
\text { (dominant), smaller benthic foraminifera } \\
\text { Accessory grains: Less than } 1 \text { percent silt to very fine sand-sized, angular, well sorted } \\
\text { quartz grains; } 1 \text {-2 percent silt to medium sand-sized black phosphorite grains above } \\
\text { through to top of cycle } \\
\text { Porosity and permeability: } 1 \text { percent interparticle porosity, } 3 \text { percent intraparticle } \\
\text { porosity, } 6 \text { percent fossil moldic porosity, } 2 \text { percent vuggy porosity; } 12 \text { percent total } \\
\text { porosity and low permeability } \\
\text { Depositional environment: Outer ramp } \\
\text { Comments: Top of cycle at } 621.6 \text { ft bls (driller’s depth) and capped by a firmground }\end{array}$ \\
\hline
\end{tabular}




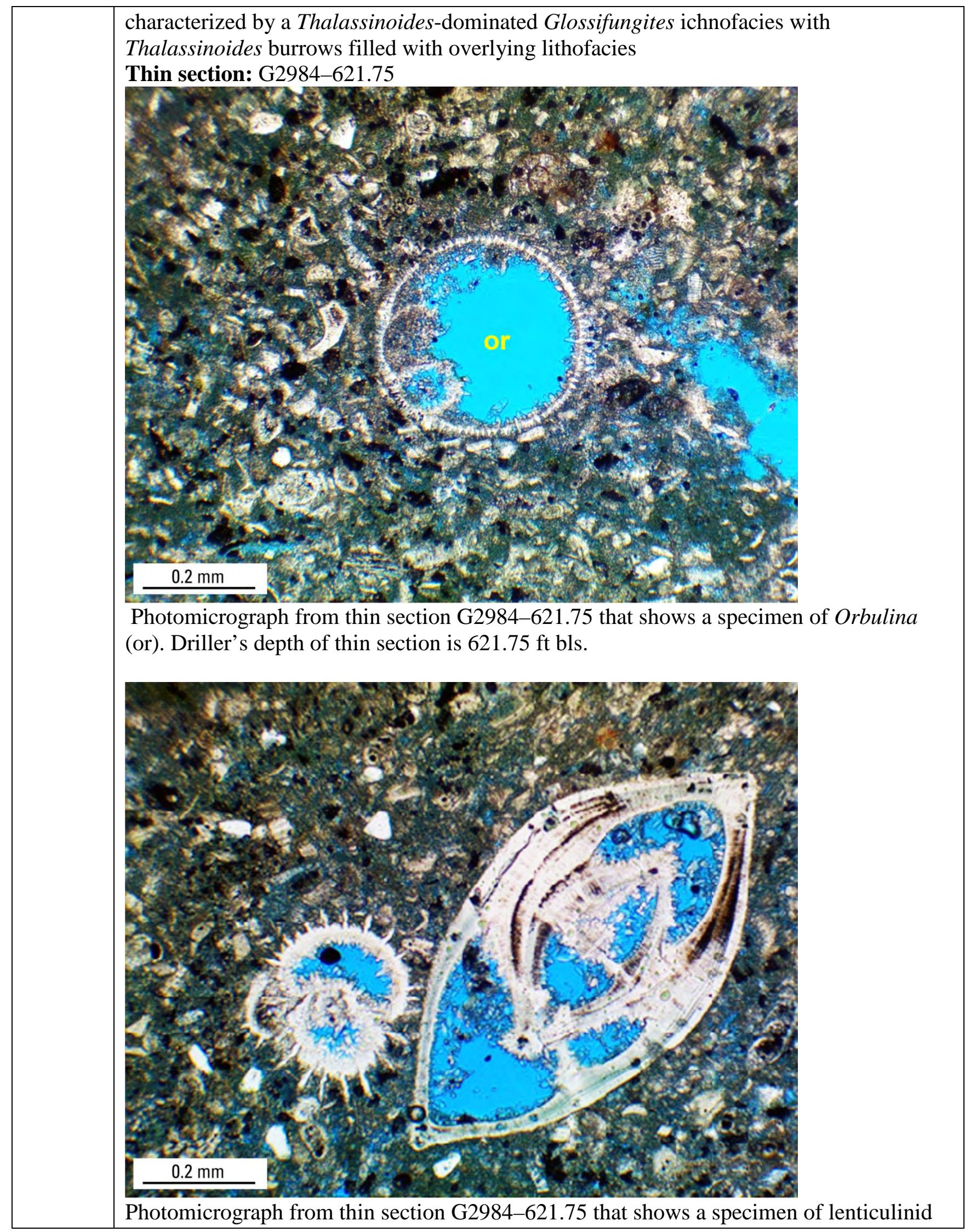




\begin{tabular}{|c|c|}
\hline & $\begin{array}{l}\text { specimen on right side) and a spinose planktic foraminifer to the left. Driller's } \\
\text { of thin section is } 621.75 \mathrm{ft} \text { bls. }\end{array}$ \\
\hline $\begin{array}{l}\text { obi } \\
\text { depth: } \\
622.0- \\
623.15 \\
\text { ft bls } \\
\\
\text { Driller's } \\
\text { depth: } \\
622.1- \\
623.24 \\
\text { ft bls }\end{array}$ & $\begin{array}{l}\text { Lithofacies: Foraminifer mudstone to packstone } \\
\text { Depositional texture: Smaller benthic and globular planktic foraminifer mudstone to } \\
\text { mud- to grain-dominated packstone } \\
\text { Color: Yellowish gray } 5 Y \text { Y } 8 \text { 1 } \\
\text { Sedimentary structures: Burrow mottled } \\
\text { Trace fossils: None identified } \\
\text { Ichnofabrics: Ichnofabric index } 5 \\
\text { Ichnofacies: Cruziana? } \\
\text { Carbonate grains: Mainly silt to very fine sand-sized angular skeletal fragments, smaller } \\
\text { benthic foraminifera, globular planktic foraminifera and minor ostracods, echinoid spines, } \\
\text { bivalve fragments. Foraminifera observed in thin section G2984-622.85 include Orbulina } \\
\text { suturalis?, Praeorbulina glomerosa s.l., Globoquadrina sp., unidentified planktic } \\
\text { foraminifera, bolivinids, lenticulinids, planktic foraminifera (dominant), smaller benthic } \\
\text { foraminifera } \\
\text { Accessory grains: } 1-10 \text { percent silt to very fine sand-sized, angular, well sorted quartz } \\
\text { grains; } 5 \text {-7 percent silt to very fine to very coarse sand-sized phosphorite grains } \\
\text { Porosity and permeability: } 1 \text {-10 percent interparticle porosity, 1-3 percent intraparticle } \\
\text { porosity, less than } 1 \text { percent fossil moldic porosity; } 2-12 \text { percent total porosity and low } \\
\text { permeability } \\
\text { Depositional environment: Outer ramp } \\
\text { Thin section: G2984-622.85 }\end{array}$ \\
\hline $\begin{array}{l}\text { obi } \\
\text { depth: } \\
623.15- \\
650.5 \mathrm{ft} \\
\text { bls } \\
\text { Driller's } \\
\text { depth: } \\
623.24- \\
650.5 \mathrm{ft} \\
\text { bls }\end{array}$ & $\begin{array}{l}\text { Lithofacies: Foraminifer wackestone and packstone } \\
\text { Depositional texture: Smaller benthic and globular planktic foraminifer wackestone and } \\
\text { mud- and grain-dominated packstone } \\
\text { Color: Yellowish gray 5Y 8/1 } \\
\text { Sedimentary structures: Burrow mottled } \\
\text { Trace fossils: Thalassinoides, Asterosoma, Ophiomorpha nodosa } \\
\text { Ichnofabrics: Ichnofabric index } 5 \\
\text { Ichnofacies: Cruziana and a thin Glossifungites ichnofacies capping the cycle } \\
\text { Carbonate grains: Mainly silt to very fine sand-sized angular skeletal fragments, smaller } \\
\text { benthic foraminifera, globular planktic foraminifera and minor ostracods, echinoid spines, } \\
\text { bivalve fragments. Foraminifera observed in thin section G2984-624.07 include } \\
\text { Praeorbulina glomerosa s.l.?, Globoquadrina sp., Globigerinoides gr. trilobus, } \\
\text { unidentified planktic foraminifera, bolivinids, smaller benthic foraminifera (dominant). } \\
\text { Foraminifera observed in thin section G2984-632.68 include Praeorbulina glomerosa s.l., } \\
\text { Globigerinoides gr. trilobus, unidentified planktic foraminifera, bolivinids, planktic } \\
\text { foraminifera, smaller benthic foraminifera (dominant). Foraminifera observed in thin } \\
\text { section G2984-644.87 include Globoquadrina sp., Globigerinoides gr. trilobus, } \\
\text { unidentified planktic foraminifera, bolivinids, lenticulinids, planktic foraminifera, smaller } \\
\text { benthic foraminifera (dominant) } \\
\text { Accessory grains: 1-10 percent silt to very fine sand-sized, angular, well sorted quartz } \\
\text { grains; } 5 \text {-7 percent silt to very fine to very coarse sand-sized phosphorite grains over } \\
\text { lower } 1.5 \text { ft of cycle; } 1 \text {-2 percent silt to medium sand-sized black phosphorite grains }\end{array}$ \\
\hline
\end{tabular}




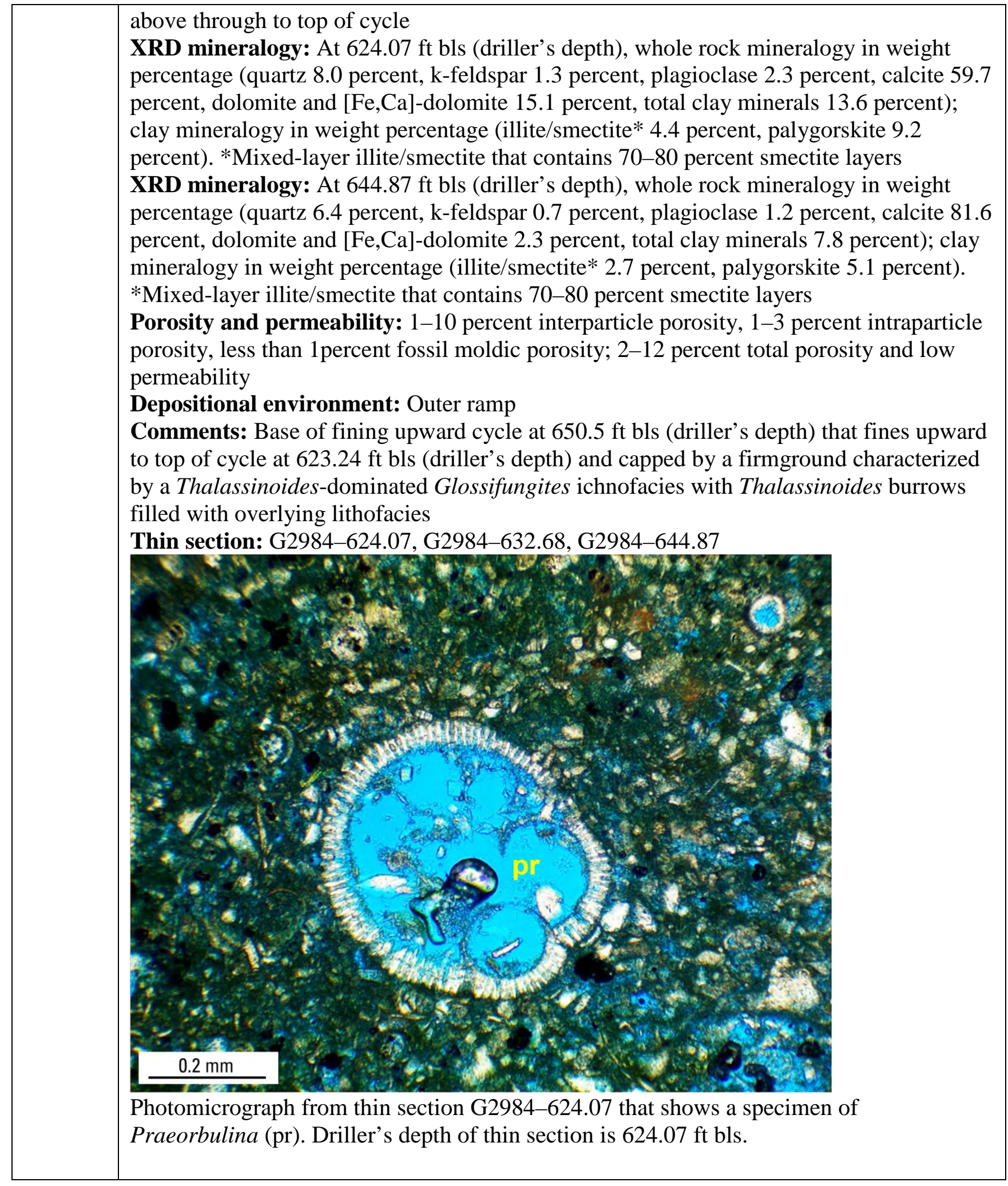




\begin{tabular}{|c|c|}
\hline & 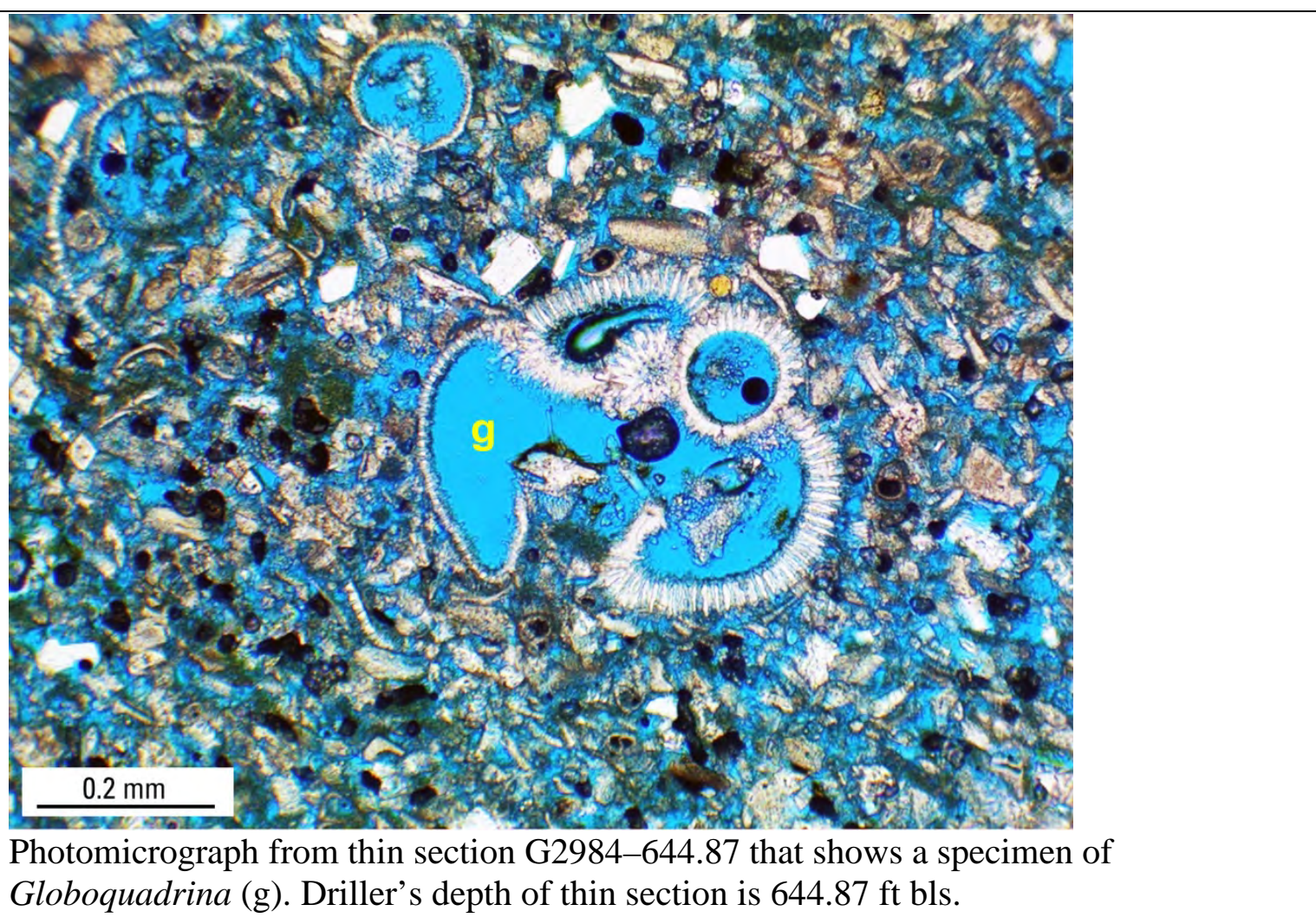 \\
\hline $\begin{array}{l}\text { obi } \\
\text { depth: } \\
650.5- \\
658.2 \mathrm{ft} \\
\text { bls } \\
\\
\text { Driller's } \\
\text { depth: } \\
650.5- \\
658.2 \mathrm{ft} \\
\text { bls }\end{array}$ & $\begin{array}{l}\text { Lithofacies: Foraminifer wackestone } \\
\text { Depositional texture: Smaller benthic foraminifer and globular planktic foraminifer } \\
\text { mudstone to wackestone } \\
\text { Color: Yellowish gray 5Y 7/2 } \\
\text { Sedimentary structures: Burrow mottled } \\
\text { Trace fossils: Thalassinoides, Zoophycos, Rhizocorallium?, Asterosoma?, Terebellina } \\
\text { (Schaubcylindrichnus)? } \\
\text { Ichnofabrics: Ichnofabric index } 5 \\
\text { Ichnofacies: Cruziana or Zoophycos with a Glossifungites ichnofacies cap } \\
\text { Carbonate grains: Mainly silt-sized and very fine sand-sized carbonate skeletal } \\
\text { fragments, smaller benthic foraminifera, globular planktic foraminifera, ostracods, bivalve } \\
\text { fragments. Foraminifera observed in thin section G2984-658.07 include Praeorbulina } \\
\text { glomerosa s.l., Globoquadrina sp., Globigerinoides gr. trilobus, unidentified planktic } \\
\text { foraminifera, bolivinids (rare), rotaliids, planktic foraminifera (dominant), smaller benthic } \\
\text { foraminifera present with lenticulinids (dominant) } \\
\text { Accessory grains: Less than } 1 \text { percent silt to fine sand-sized, angular, well sorted quartz } \\
\text { grains; } 1-2 \text { percent silt to very fine sand-sized black grains (mainly phosphorite) } \\
\text { XRD mineralogy: At } 654.15 \text { ft bls (driller's depth), whole rock mineralogy in weight } \\
\text { percentage (quartz } 5.4 \text { percent, calcite } 68.6 \text { percent, dolomite and [Fe,Ca]-dolomite } 3.1 \\
\text { percent, total clay minerals } 22.9 \text { percent); clay mineralogy in weight percentage } \\
\text { (illite/smectite* } 10.7 \text { percent, palygorskite } 12.2 \text { percent). *Mixed-layer illite/smectite that } \\
\text { contains } 70-80 \text { percent smectite layers } \\
\text { Porosity and permeability: } 1-5 \text { percent interparticle porosity, } 3 \text { percent intraparticle } \\
\text { porosity; } 4-7 \text { percent total porosity and low permeability }\end{array}$ \\
\hline
\end{tabular}




\begin{tabular}{|c|c|}
\hline & $\begin{array}{l}\text { Depositional environment: Outer ramp } \\
\text { Comments: Top of a fining upward cycle with a top at } 650.5 \mathrm{ft} \text { bls (driller's depth). } \\
\text { Upper } 2 \mathrm{ft} \text { of cycle is a Thalassinoides-dominated Glossifungites ichnofacies, and major } \\
\text { lithofacies shift across cycle top. The Glossifungites ichnofacies associates with a } \\
\text { firmground capping the cycle } \\
\text { Thin section: G2984-658.07 }\end{array}$ \\
\hline $\begin{array}{l}\text { obi } \\
\text { depth: } \\
658.2- \\
659.1 \mathrm{ft} \\
\text { bls } \\
\\
\text { Driller's } \\
\text { depth: } \\
658.2- \\
659.1 \mathrm{ft} \\
\text { bls }\end{array}$ & $\begin{array}{l}\text { Lithofacies: Foraminifer wackestone to packstone } \\
\text { Depositional texture: Smaller benthic foraminifer and globular planktic foraminifer } \\
\text { wackestone and mud-dominated packstone } \\
\text { Color: Yellowish gray 5Y 8/1 } \\
\text { Sedimentary structures: Burrow mottled } \\
\text { Trace fossils: Thalassinoides, Asterosoma } \\
\text { Ichnofabrics: Ichnofabric index } 5 \\
\text { Ichnofacies: Cruziana } \\
\text { Carbonate grains: Mainly smaller benthic foraminifera (including lenticulinids } \\
\text { [dominant], rotaliids), globular planktic foraminifera, and silt to very fine sand-sized } \\
\text { angular skeletal fragments; minor ostracods, echinoid spines, bivalve fragments } \\
\text { Accessory grains: } 1 \text { percent silt to very fine sand-sized, angular, well sorted quartz } \\
\text { grains; } 5-7 \text { percent silt to small pebble-sized black phosphorite grains } \\
\text { Porosity and permeability: } 1 \text { percent interparticle porosity, } 1 \text { percent intraparticle } \\
\text { porosity; } 2 \text { percent total porosity and low permeability } \\
\text { Depositional environment: Outer ramp } \\
\text { Comments: Base of fining upward cycle at } 659.1 \mathrm{ft} \text { bls (driller's depth) that fines upward } \\
\text { to top of cycle at } 650.5 \mathrm{ft} \text { bls (driller's depth) and capped by a Thalassinoides-dominated } \\
\text { Glossifungites ichnofacies. Upper surface of cycle is a firmground }\end{array}$ \\
\hline $\begin{array}{l}\text { obi } \\
\text { depth: } \\
659.1- \\
659.9 \mathrm{ft} \\
\text { bls } \\
\\
\text { Driller's } \\
\text { depth: } \\
659.1- \\
659.9 \mathrm{ft} \\
\text { bls }\end{array}$ & $\begin{array}{l}\text { Lithofacies: Quartz sand (caved from above in borehole) } \\
\text { Depositional texture: Quartz sand sourced from the Peace River Formation } \\
\text { Color: Light gray N7 } \\
\text { Accessory grains: } 90 \text { percent silt to very fine sand-sized, angular, well sorted quartz } \\
\text { grains; } 10 \text { percent silt to fine sand-sized black grains (mainly phosphorite) } \\
\text { Porosity and permeability: } 20 \text { percent interparticle and total porosity; moderate } \\
\text { permeability } \\
\text { Depositional environment: Outer ramp } \\
\text { Comments: Regressive quartz sand(?). Observations weigh towards this quartz sand } \\
\text { sourced from the Peace River Formation above. No quartz sand was observed on OBI-40 } \\
\text { image log data of good quality at or near this depth interval and was not observed on the } \\
\text { ABI image log data. No increase in borehole diameter to indicate washout of quartz sand } \\
\text { across this interval—quartz sand recovered was "poured" into core box from core barrel } \\
\text { with no evidence of in situ firmness. The well as cased to a driller's depth about } 468 \mathrm{ft} \text { bls } \\
\text { at the time this interval was cored, so there was ample very fine quartz sand of the Peace } \\
\text { River Formation exposed along the borehole wall at the time this interval was drilled. The } \\
\text { quartz sand in this interval occurs at the top of a core run, where caved sand can be } \\
\text { recovered, if present at the bottom of a corehole. Also, composition of this quartz sand is } \\
\text { identical to quartz sand of the Peace River Formation that was exposed along the borehole } \\
\text { wall during coring of this interval }\end{array}$ \\
\hline
\end{tabular}




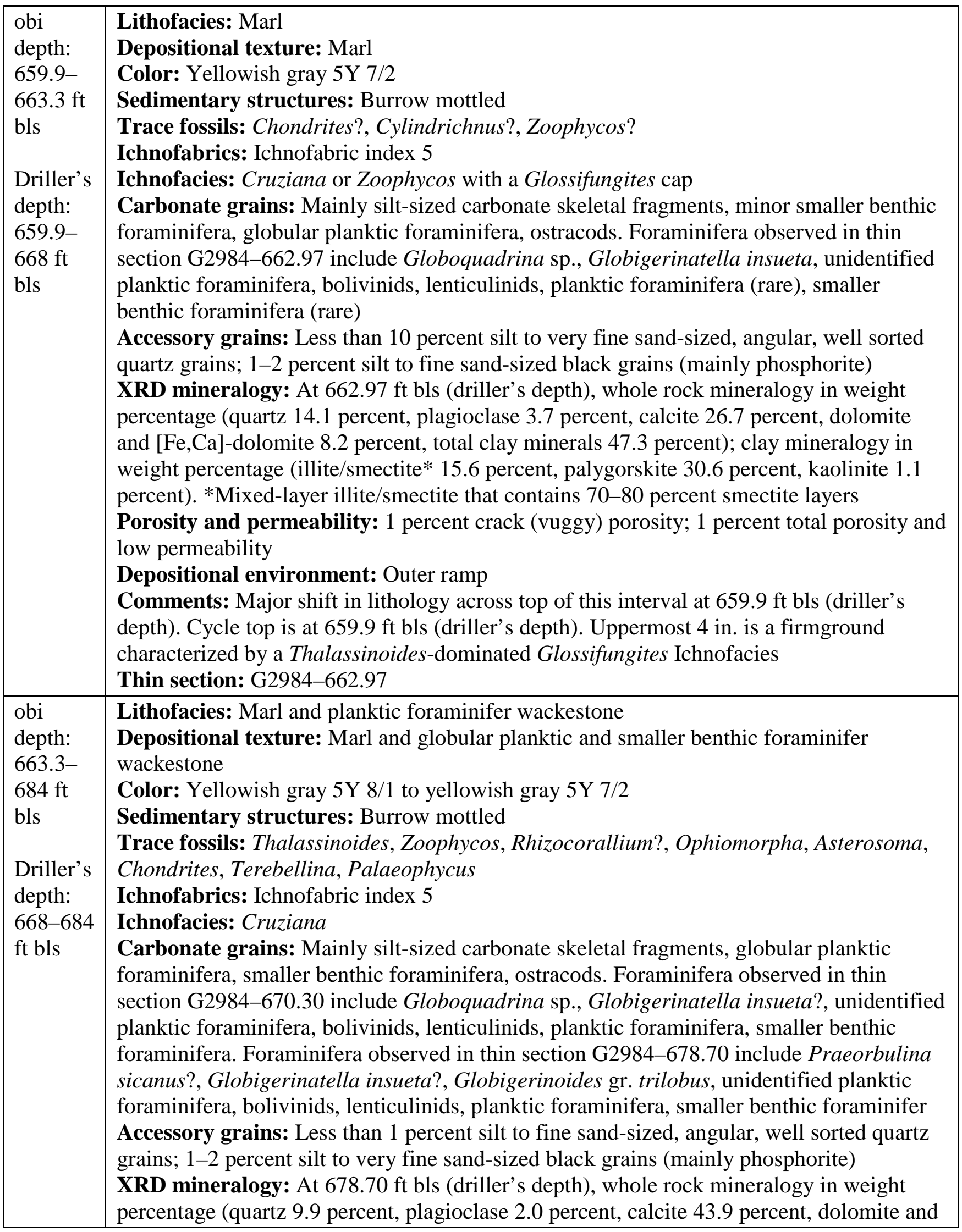




\begin{tabular}{|c|c|}
\hline & $\begin{array}{l}\text { [Fe,Ca]-dolomite } 7.1 \text { percent, total clay minerals } 37.1 \text { percent); clay mineralogy in weight } \\
\text { percentage (illite/smectite* } 12.4 \text { percent, palygorskite } 23.8 \text { percent, kaolinite } 0.9 \text { percent). } \\
\text { *Mixed-layer illite/smectite that contains } 70-80 \text { percent smectite layers } \\
\text { Porosity and permeability: } 1 \text { percent interparticle porosity, } 1 \text { percent intraparticle } \\
\text { porosity; } 2 \text { percent total porosity and low permeability } \\
\text { Depositional environment: Outer ramp } \\
\text { Thin section: G2984-670.30, G2984-678.70 }\end{array}$ \\
\hline $\begin{array}{l}\text { obi } \\
\text { depth: } \\
684- \\
688.25 \\
\text { ft bls } \\
\\
\text { Driller's } \\
\text { depth: } \\
684- \\
688.25 \\
\text { ft bls }\end{array}$ & $\begin{array}{l}\text { Lithofacies: Planktic foraminifer wackestone and packstone } \\
\text { Depositional texture: Globular planktic and smaller benthic foraminifer wackestone and } \\
\text { mud-dominated packstone } \\
\text { Color: Yellowish gray } 5 Y \text { Y } 8 \\
\text { Sedimentary structures: Burrow mottled } \\
\text { Trace fossils: Thalassinoides, Taenidium, Rhizocorallium (at } 687.5 \mathrm{ft} \text { bls driller's depth) } \\
\text { Ichnofabrics: Ichnofabric index } 5 \\
\text { Ichnofacies: Cruziana or Zoophycos } \\
\text { Carbonate grains: Mainly globular planktic foraminifera, smaller benthic foraminifera } \\
\text { (including rotaliids), silt to very fine sand-sized angular skeletal fragments and minor } \\
\text { ostracods, echinoid spines and plates. Foraminifera observed in thin section G2984- } \\
\text { 686.70 include Praeorbulina glomerosa s.l., Globoquadrina sp., Globigerinatella } \\
\text { insueta?, unidentified planktic foraminifera, bolivinids, lenticulinids, rotaliids, planktic } \\
\text { foraminifera, smaller benthic foraminifera } \\
\text { Accessory grains: } 1 \text { percent silt to very fine sand-sized, angular, well sorted quartz } \\
\text { grains; } 5 \text { percent silt to small pebble-sized black phosphorite grains } \\
\text { Porosity and permeability: } 3 \text { percent interparticle porosity, } 3 \text { percent intraparticle } \\
\text { porosity; } 6 \text { percent total porosity and low permeability } \\
\text { Depositional environment: Outer ramp } \\
\text { Comments: Base of fining upward cycle at } 688.25 \text { ft bls (driller's depth) that fines } \\
\text { upward to top of cycle at } 659.9 \text { ft bls (driller's depth) } \\
\text { Thin section: G2984-686.70 }\end{array}$ \\
\hline $\begin{array}{l}\text { obi } \\
\text { depth: } \\
688.25- \\
693.45 \\
\text { ft bls } \\
\text { Driller's } \\
\text { depth: } \\
688.25- \\
694.25 \\
\text { ft bls }\end{array}$ & $\begin{array}{l}\text { Lithofacies: Planktic foraminifer wackestone } \\
\text { Depositional texture: Globular planktic and smaller benthic foraminifer wackestone } \\
\text { Color: Yellowish gray } 5 Y \text { Y } 8 \text { to yellowish gray } 5 Y \text { Y/2 } \\
\text { Sedimentary structures: Mainly burrow mottled, minor thinly laminated to very thinly } \\
\text { bedded } \\
\text { Trace fossils: Thalassinoides, Zoophycos, Taenidium?, Rhizocoralliium? (694 ft bls } \\
\text { driller's depth) } \\
\text { Ichnofabrics: Ichnofabric index } 1-5 \text {, mostly } 5 \\
\text { Ichnofacies: Cruziana or Zoophycos with a Glossifungites ichnofacies capping the cycle } \\
\text { Carbonate grains: Mainly silt-sized carbonate skeletal fragments, globular planktic } \\
\text { foraminifera, smaller benthic foraminifera } \\
\text { Accessory grains: Less than } 1 \text { percent silt to fine sand-sized, angular, well sorted quartz } \\
\text { grains; } 1-2 \text { percent silt to very fine sand-sized black grains (mainly phosphorite) } \\
\text { XRD mineralogy: At } 689.90 \mathrm{ft} \text { bls (driller's depth), whole rock mineralogy in weight } \\
\text { percentage (quartz } 4.9 \text { percent, calcite } 64.1 \text { percent, dolomite and [Fe,Ca]-dolomite } 3.3 \\
\text { percent, fluorapatite } 1.1 \text { percent, total clay minerals } 26.5 \text { percent); clay mineralogy in } \\
\text { weight percentage (illite/smectite* } 12.4 \text { percent, palygorskite } 14.1 \text { percent). *Mixed-layer }\end{array}$ \\
\hline
\end{tabular}




\begin{tabular}{|c|c|}
\hline & $\begin{array}{l}\text { illite/smectite that contains } 70-80 \text { percent smectite layers } \\
\text { XRD mineralogy: At } 691.00 \mathrm{ft} \text { bls (driller's depth), whole rock mineralogy in weight } \\
\text { percentage (quartz } 4.7 \text { percent, calcite } 70.3 \text { percent, dolomite and [Fe,Ca]-dolomite } 2.6 \\
\text { percent, total clay minerals } 22.4 \text { percent); clay mineralogy in weight percentage } \\
\text { (illite/smectite* } 7.2 \text { percent, palygorskite } 15.2 \text { percent). *Mixed-layer illite/smectite that } \\
\text { contains } 70-80 \text { percent smectite layers } \\
\text { Porosity and permeability: } 3 \text { percent interparticle porosity, } 3 \text { percent intraparticle } \\
\text { porosity; } 6 \text { percent total porosity and low permeability } \\
\text { Depositional environment: Outer ramp } \\
\text { Comments: Uppermost part of cycle is a Thalassinoides-dominated Glossifungites } \\
\text { ichnofacies with sediment from overlying cycle piping down into Thalassinoides burrows. } \\
\text { Uppermost part of cycle is a firmground. Cycle top is at } 688.25 \mathrm{ft} \text { bls (driller's depth). } \\
\text { Glossifungites ichnofacies extends downward about } 2 \mathrm{ft} \text { from cycle top. Upper surface of } \\
\text { cycle is a firmground }\end{array}$ \\
\hline $\begin{array}{l}\text { obi } \\
\text { depth: } \\
693.45- \\
696.5 \mathrm{ft} \\
\text { bls } \\
\text { Driller's } \\
\text { depth: } \\
694.25- \\
697.3 \mathrm{ft} \\
\text { bls }\end{array}$ & $\begin{array}{l}\text { Lithofacies: Planktic foraminifer wackestone and packstone } \\
\text { Depositional texture: Globular planktic and smaller benthic foraminifer wackestone and } \\
\text { mud-dominated packstone } \\
\text { Color: Yellowish gray } 5 Y \text { Y } 1 \\
\text { Sedimentary structures: Burrow mottled } \\
\text { Trace fossils: Thalassinoides, Taenidium } \\
\text { Ichnofabrics: Ichnofabric index } 5 \\
\text { Ichnofacies: Cruziana or Zoophycos } \\
\text { Carbonate grains: Mainly globular planktic foraminifera, smaller benthic foraminifera, } \\
\text { silt to very fine sand-sized angular skeletal fragments and minor ostracods, echinoid } \\
\text { spines and plates } \\
\text { Accessory grains: } 1 \text { percent silt to very fine sand-sized, angular, well sorted quartz } \\
\text { grains; } 5 \text {-7 percent silt to small pebble-sized black phosphorite grains } \\
\text { XRD mineralogy: At } 696.00 \text { ft bls (driller's depth), whole rock mineralogy in weight } \\
\text { percentage (quartz } 2.0 \text { percent, calcite } 79.8 \text { percent, dolomite and [Fe,Ca]-dolomite } 1.1 \\
\text { percent, fluorapatite } 2.8 \text { percent, total clay minerals } 14.3 \text { percent); clay mineralogy in } \\
\text { weight percentage (illite/smectite* } 4.3 \text { percent, palygorskite } 10.0 \text { percent). *Mixed-layer } \\
\text { illite/smectite that contains } 70-80 \text { percent smectite layers } \\
\text { Porosity and permeability: } 3 \text { percent interparticle porosity, } 3 \text { percent intraparticle } \\
\text { porosity; } 6 \text { percent total porosity and low permeability } \\
\text { Depositional environment: Outer ramp } \\
\text { Comments: Base of fining upward cycle at } 697.3 \text { ft bls (driller's depth) that fines upward } \\
\text { to top of cycle at } 688.25 \mathrm{ft} \text { bls (driller's depth) }\end{array}$ \\
\hline $\begin{array}{l}\text { obi } \\
\text { depth: } \\
696.5- \\
698.8 \mathrm{ft} \\
\text { bls }\end{array}$ & $\begin{array}{l}\text { Lithofacies: Marl } \\
\text { Depositional texture: Marl } \\
\text { Color: Yellowish gray } 5 Y \text { 8/1 to yellowish gray } 5 Y \text { 7/2 } \\
\text { Sedimentary structures: Mainly burrow mottled, minor thinly laminated to thickly } \\
\text { laminated } \\
\text { Trace fossils: Thalassinoides } \\
\text { Ichnofabrics: Ichnofabric index 1-5, mostly } 5 \\
\text { Ichnofacies: Cruziana or Zoophycos with a Glossifungites ichnofacies capping the cycle } \\
\text { Carbonate grains: Mainly silt-sized carbonate skeletal fragments, globular planktic }\end{array}$ \\
\hline
\end{tabular}




\begin{tabular}{|c|c|}
\hline & $\begin{array}{l}\text { foraminifera, smaller benthic foraminifera. Foraminifera observed in thin section G2984- } \\
698.64 \text { include Praeorbulina glomerosa s.l.?, Globigerinoides gr. trilobus, bolivinids, } \\
\text { planktic foraminifera (rare), smaller benthic foraminifera (rare) } \\
\text { Accessory grains: Less than } 1 \text { percent silt to very fine sand-sized, angular, well sorted } \\
\text { quartz grains; } 1 \text {-2 percent silt to fine sand-sized black grains (mainly phosphorite) } \\
\text { XRD mineralogy: At } 698.64 \text { ft bls (driller's depth), whole rock mineralogy in weight } \\
\text { percentage (quartz } 3.8 \text { percent, plagioclase } 1.1 \text { percent, calcite } 48.2 \text { percent, dolomite and } \\
\text { [Fe,Ca]-dolomite } 10.6 \text { percent, total clay minerals } 36.3 \text { percent); clay mineralogy in } \\
\text { weight percentage (illite/smectite* } 12.3 \text { percent, palygorskite } 24.0 \text { percent). *Mixed-layer } \\
\text { illite/smectite that contains } 70-80 \text { percent smectite layers } \\
\text { Porosity and permeability: } 1 \text { percent interparticle porosity, } 1 \text { percent intraparticle } \\
\text { porosity; } 2 \text { percent total porosity and low permeability } \\
\text { Depositional environment: Outer ramp } \\
\text { Comments: Uppermost part of cycle is a Thalassinoides-dominated Glossifungites } \\
\text { ichnofacies with sediment from overlying cycle piping down into Thalassinoides burrows. } \\
\text { Uppermost part of cycle is a firmground. Cycle top is at } 697.3 \mathrm{ft} \text { bls (driller's depth). } \\
\text { Glossifungites ichnofacies extends downward about } 2 \mathrm{ft} \text { from cycle top. Upper surface of } \\
\text { cycle is a firmground } \\
\text { Thin section: G2984-698.64 }\end{array}$ \\
\hline $\begin{array}{l}\text { obi } \\
\text { depth: } \\
698.8- \\
710 \mathrm{ft} \\
\text { bls }\end{array}$ & $\begin{array}{l}\text { Lithofacies: Planktic foraminifer mudstone and wackestone } \\
\text { Depositional texture: Globular planktic and smaller benthic foraminifer mudstone and } \\
\text { wackestone } \\
\text { Color: Yellowish gray } 5 Y \text { 8/1 to yellowish gray } 5 Y \text { Y/2 } \\
\text { Sedimentary structures: Mainly burrow mottled, minor thinly laminated to very thinly } \\
\text { bedded }\end{array}$ \\
\hline $\begin{array}{l}\text { Driller’s } \\
\text { depth: } \\
699.6- \\
712 \mathrm{ft} \\
\text { bls }\end{array}$ & $\begin{array}{l}\text { Trace fossils: Thalassinoides, Zoophycos (708 ft bls [driller’s depth]), Taenidium? } \\
\text { Ichnofabrics: Ichnofabric index 1-5, mostly } 5 \\
\text { Ichnofacies: Cruziana or Zoophycos } \\
\text { Carbonate grains: Mainly silt-sized carbonate skeletal fragments, globular planktic } \\
\text { foraminifera, smaller benthic foraminifera. Foraminifera observed in thin section G2984- } \\
708.68 \text { include planktic foraminifera (including Globoquadrina sp., Globigerinoides gr. } \\
\text { trilobus), smaller benthic foraminifera (including bolivinids, lenticulinids) } \\
\text { Accessory grains: Less than } 1 \text { percent silt to fine sand-sized, angular, well sorted quartz } \\
\text { grains; } 1-2 \text { percent silt to very fine sand-sized black grains (mainly phosphorite); sponge } \\
\text { spicules } \\
\text { XRD mineralogy: At } 708.00 \text { ft bls (driller's depth), whole rock mineralogy in weight } \\
\text { percentage (quartz } 3.3 \text { percent, calcite } 76.3 \text { percent, dolomite and [Fe,Ca]-dolomite } 1.6 \\
\text { percent, total clay minerals } 18.8 \text { percent); clay mineralogy in weight percentage } \\
\text { (illite/smectite* } 7.2 \text { percent, palygorskite } 11.6 \text { percent) } * \text { Mixed-layer illite/smectite that } \\
\text { contains } 70-80 \text { percent smectite layers } \\
\text { Porosity and permeability: } 1-8 \text { percent interparticle porosity, } 1-3 \text { percent intraparticle } \\
\text { porosity; } 2-11 \text { percent total porosity and low permeability } \\
\text { Depositional environment: Outer ramp } \\
\text { Thin section: G2984-708.68 }\end{array}$ \\
\hline $\begin{array}{l}\text { obi } \\
\text { depth: }\end{array}$ & $\begin{array}{l}\text { Lithofacies: Planktic foraminifer wackestone and packstone } \\
\text { Depositional texture: Globular planktic and smaller benthic foraminifer wackeston }\end{array}$ \\
\hline
\end{tabular}




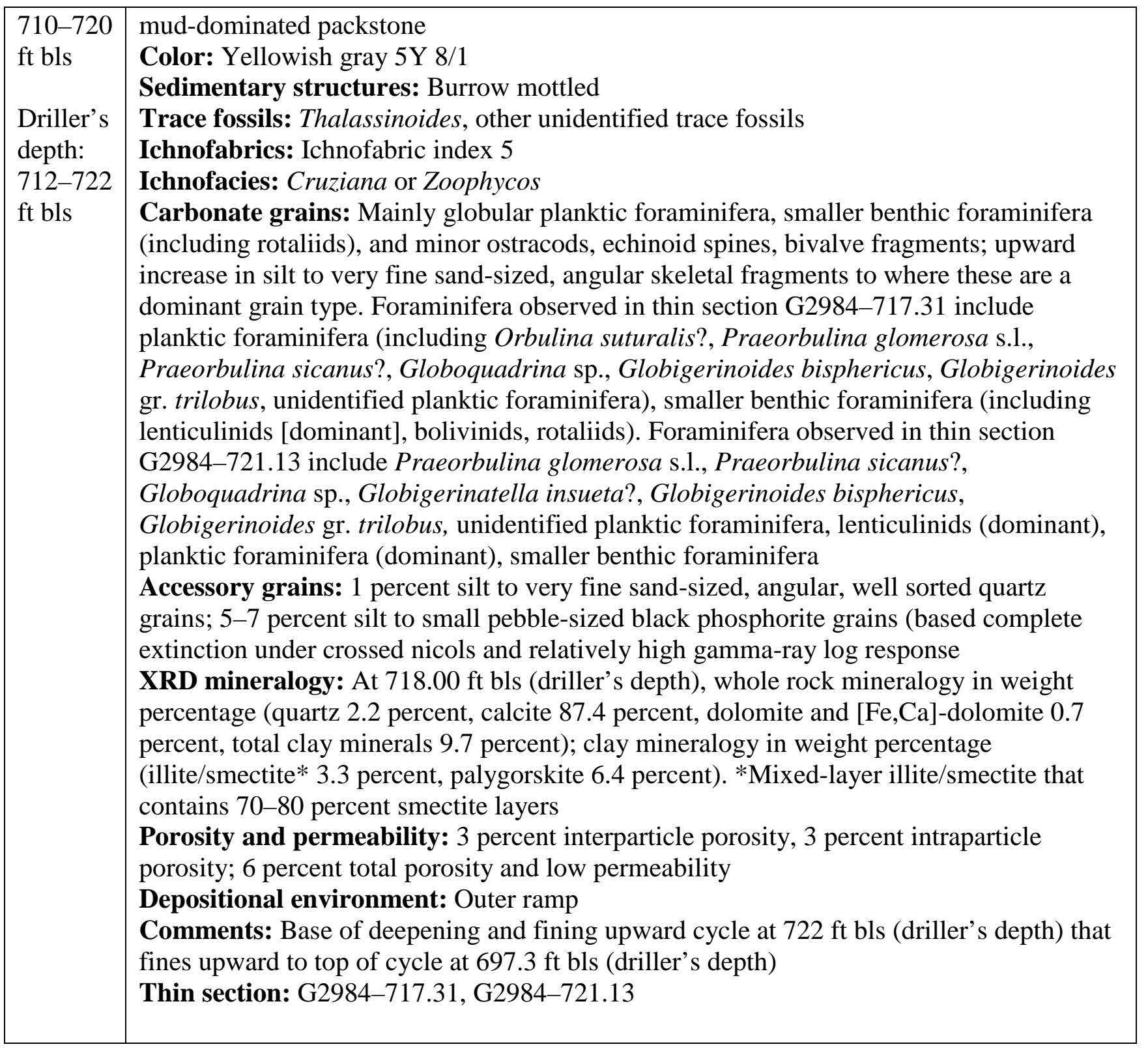




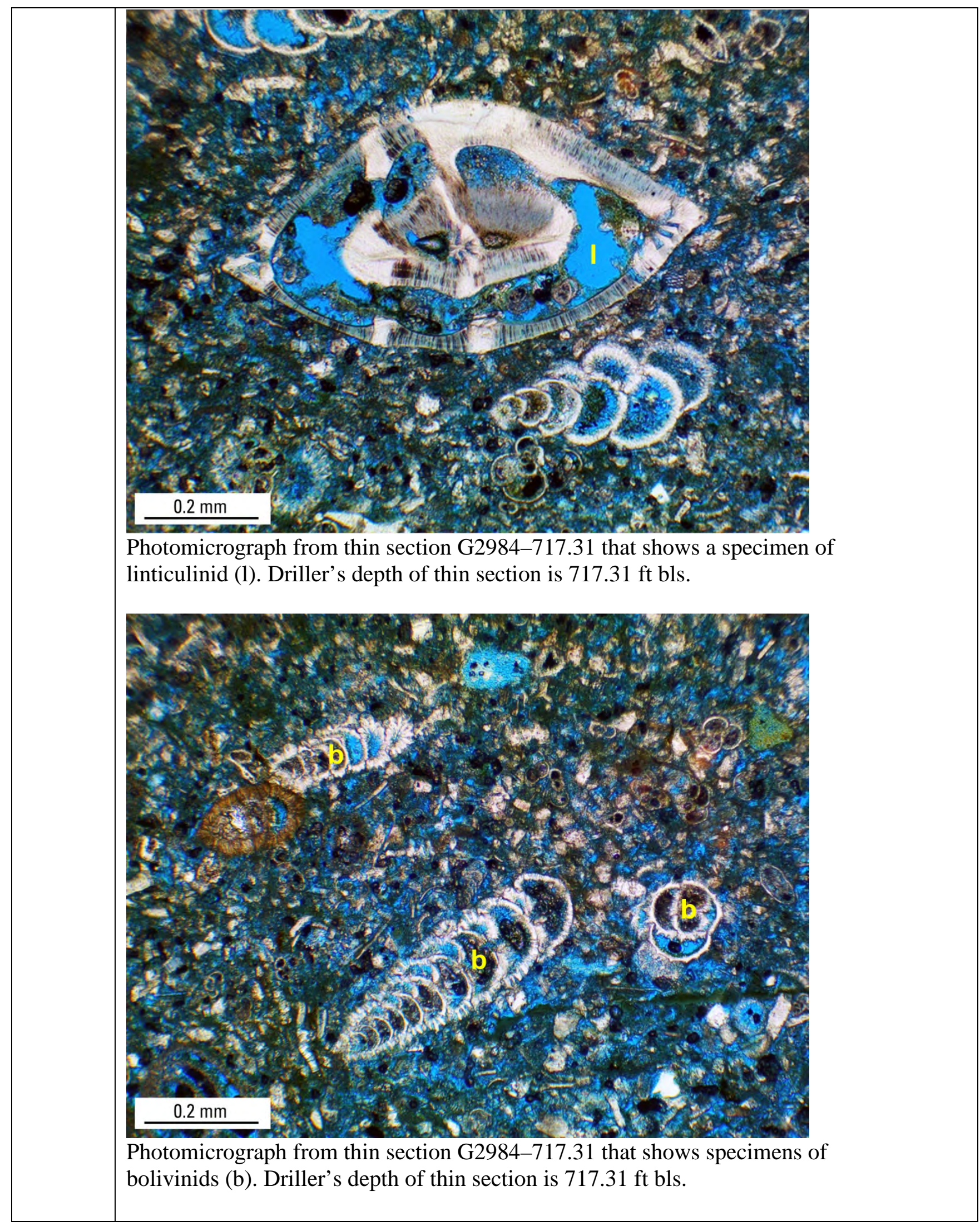




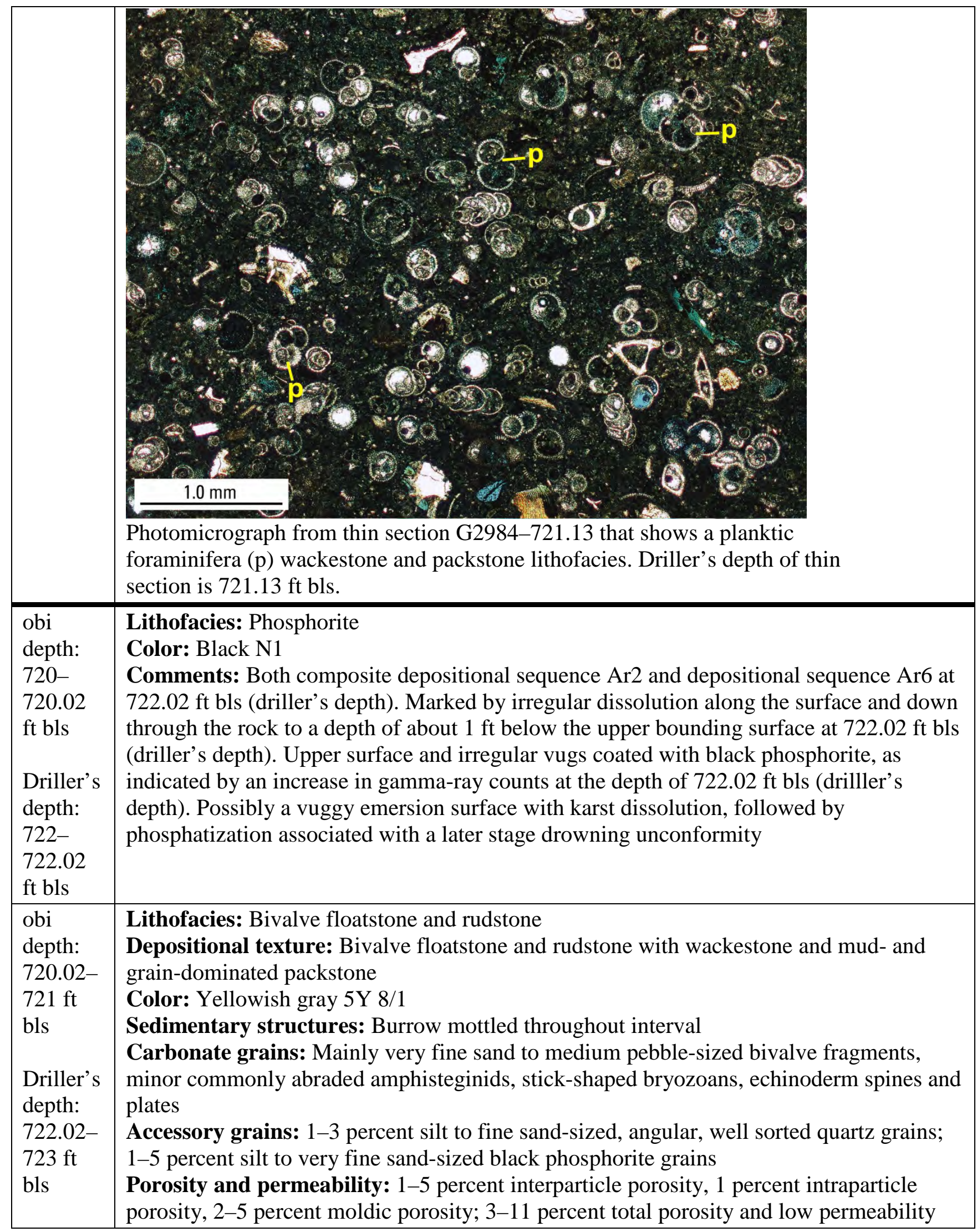




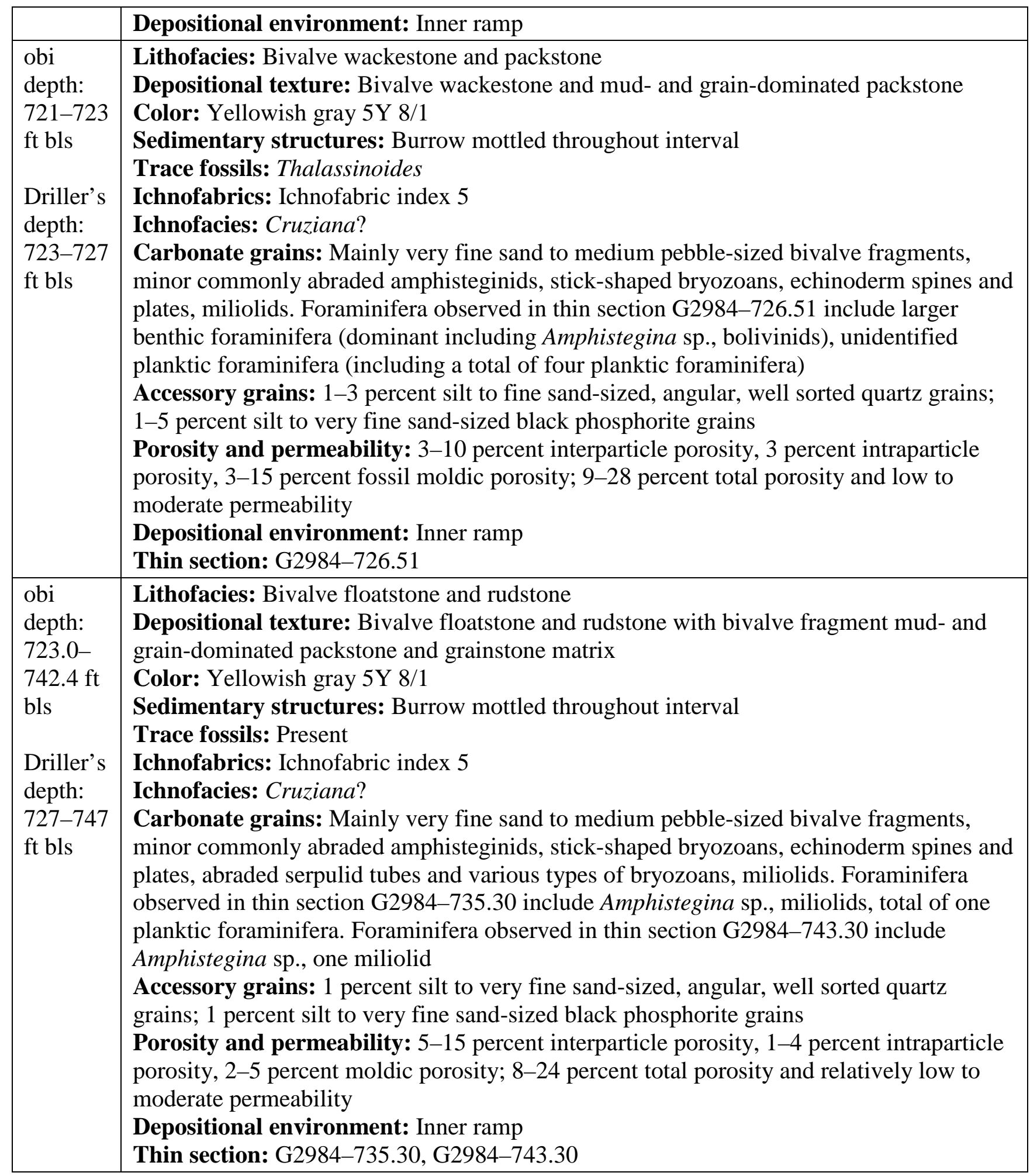




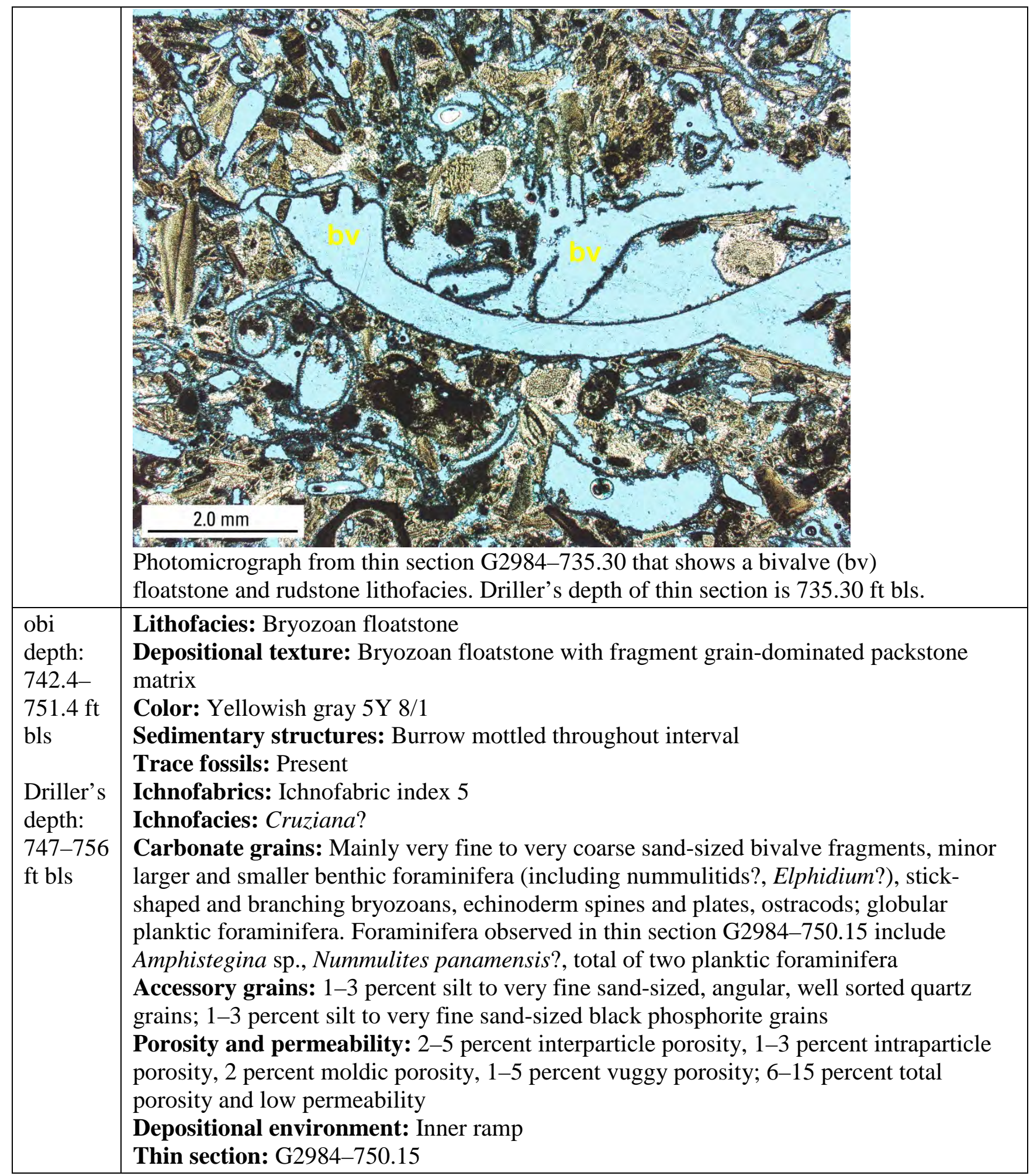




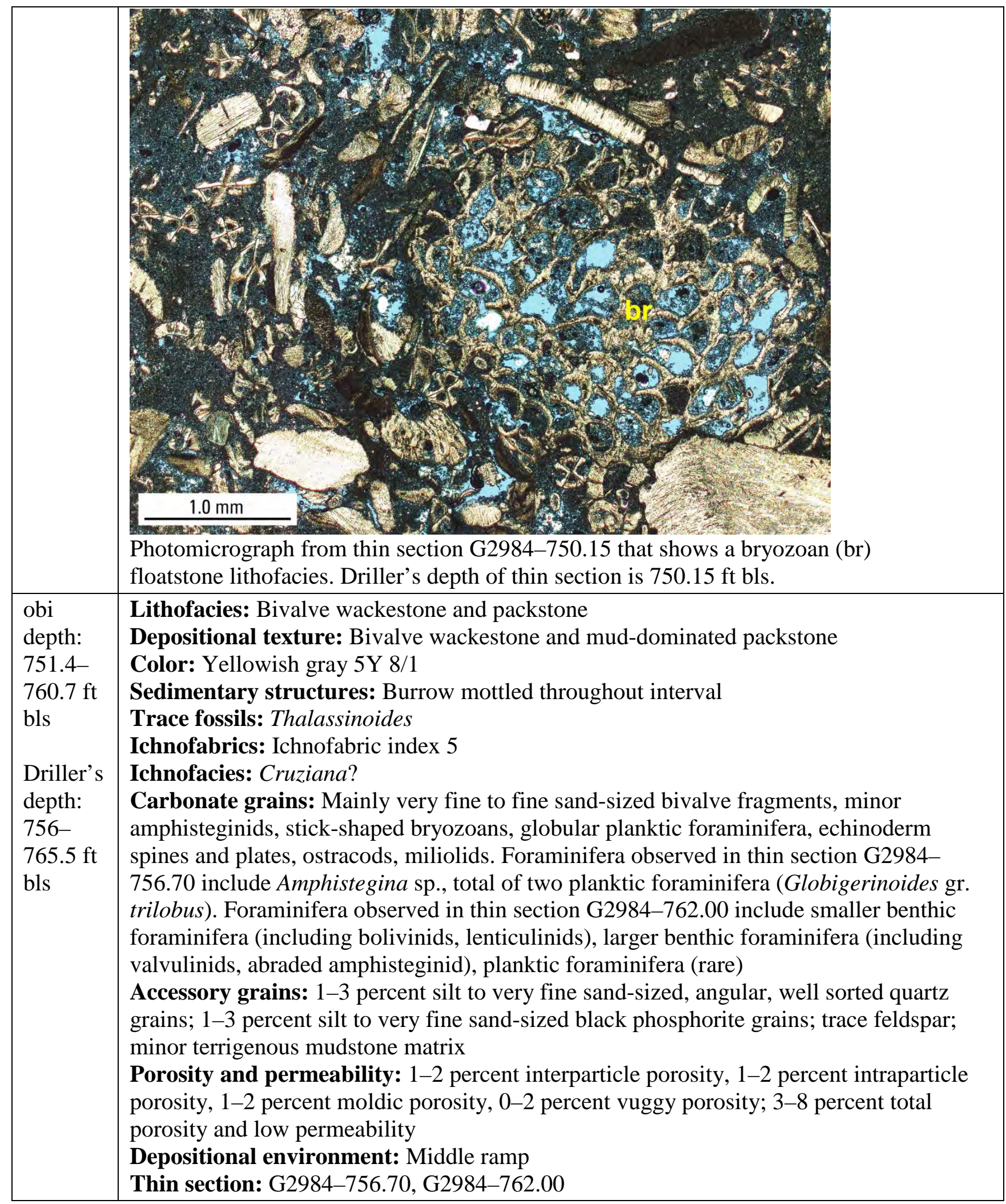




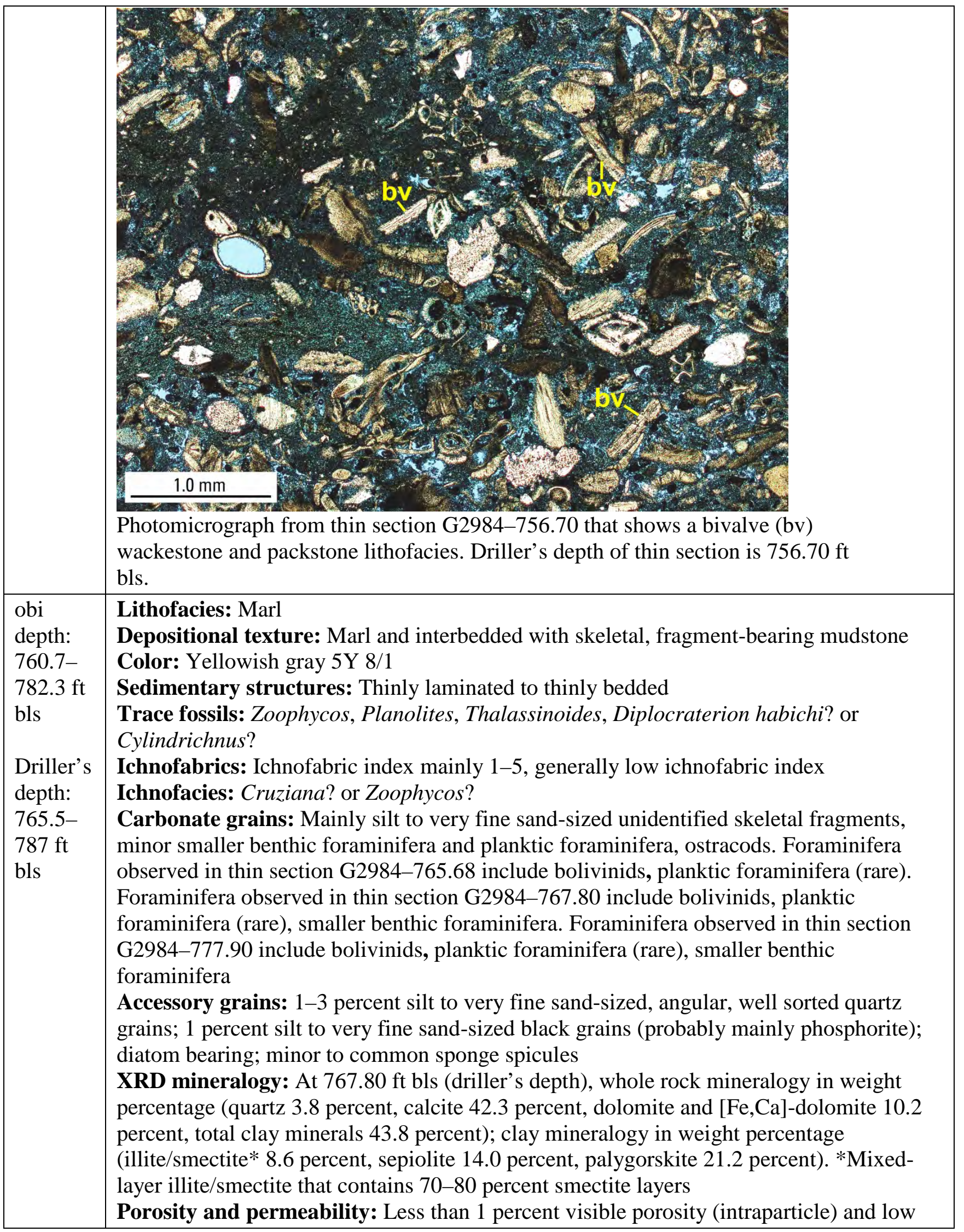




\begin{tabular}{|c|c|}
\hline & 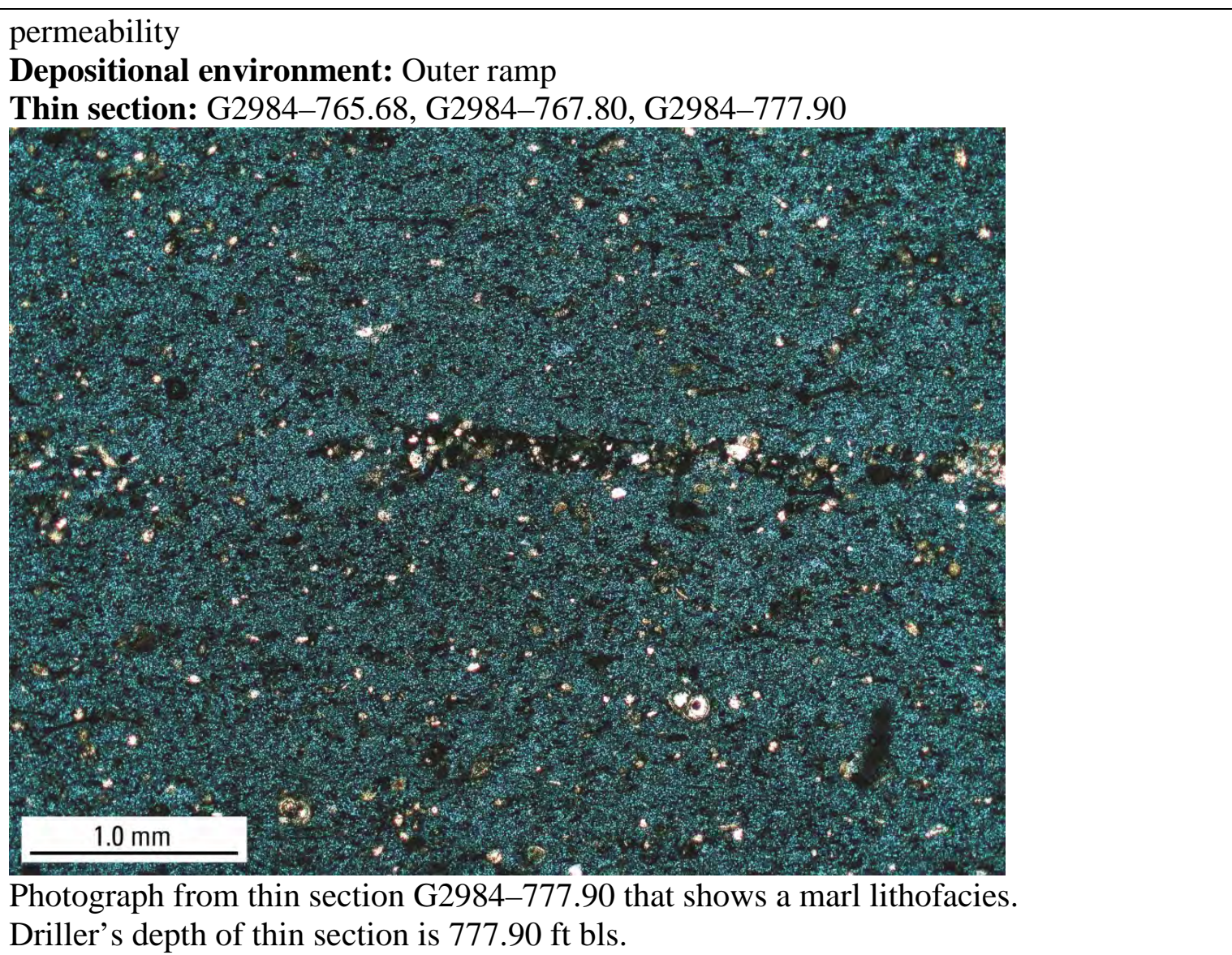 \\
\hline $\begin{array}{l}\text { obi } \\
\text { depth: } \\
782.3- \\
802.7 \mathrm{ft} \\
\text { bls } \\
\\
\text { Driller's } \\
\text { depth: } \\
787- \\
802.5 \mathrm{ft} \\
\text { bls }\end{array}$ & $\begin{array}{l}\text { Lithofacies: Diatom-bearing marl } \\
\text { Depositional texture: Diatom-bearing marl interlaminated and interbedded with smaller } \\
\text { benthic foraminifer and globular planktic foraminifer bearing marl } \\
\text { Color: Yellowish gray 5Y 8/1 } \\
\text { Sedimentary structures: Thinly laminated to thinly bedded } \\
\text { Trace fossils: Minor traces, one possible Zoophycos } \\
\text { Ichnofabrics: Ichnofabric index mainly } 1 \text {-3 } \\
\text { Ichnofacies: Not determined } \\
\text { Carbonate grains: Mainly silt to very fine sand-sized unidentified skeletal fragments, } \\
\text { very minor smaller benthic foraminifera and planktic foraminifera. Foraminifera observed } \\
\text { in thin section G2984-789.86 planktic foraminifera (rare), smaller benthic foraminifera } \\
\text { Accessory grains: } 1 \text { percent silt to very fine sand-sized, angular, well sorted quartz } \\
\text { grains; } 1 \text { percent silt to very fine sand-sized black grains (probably mainly phosphorite); } \\
\text { diatom bearing; minor sponge spicules } \\
\text { Porosity and permeability: Less than } 1 \text { percent visible porosity (intraparticle) and low } \\
\text { permeability } \\
\text { Depositional environment: Outer ramp } \\
\text { Thin section: G2984-789.86 }\end{array}$ \\
\hline $\begin{array}{l}\text { obi } \\
\text { depth: } \\
802.7- \\
804.2 \mathrm{ft}\end{array}$ & $\begin{array}{l}\text { Lithofacies: Marl interlaminated with wackestone and packstone } \\
\text { Depositional texture: Marl interlaminated with skeletal, globular planktic and smaller } \\
\text { benthic foraminifer wackestone and mud- to grain-dominated packstone } \\
\text { Color: Yellowish gray 5Y 8/1 }\end{array}$ \\
\hline
\end{tabular}




\begin{tabular}{|c|c|}
\hline $\begin{array}{l}\text { Driller’s } \\
\text { depth: } \\
802.5- \\
808.9 \mathrm{ft} \\
\text { bls }\end{array}$ & $\begin{array}{l}\text { Sedimentary structures: Burrow mottled, fining upward laminations with wackestones } \\
\text { and packstones at base and marl at top of centimeter-scale cyclic laminations } \\
\text { Trace fossils: Abundant trace fossils with homogeneous texture throughout } \\
\text { Ichnofabrics: Ichnofabric index } 5 \\
\text { Ichnofacies: Not determined } \\
\text { Carbonate grains: Mainly small fragments of bivalves, globular planktic foraminifera, } \\
\text { smaller benthic foraminifera, and silt to medium sand-sized unidentified skeletal } \\
\text { fragments; minor ostracods, echinoid spines. Foraminifera observed in thin section } \\
\text { G2984-805.35 include smaller benthic foraminifera. Foraminifera observed in thin } \\
\text { section G2984-807.95 include planktic foraminifera } \\
\text { Accessory grains: } 1-10 \text { percent silt to very fine sand-sized, angular, well sorted quartz } \\
\text { grains; } 1-2 \text { percent silt to very fine sand-sized black grains (probably mainly phosphorite) } \\
\text { XRD mineralogy: At } 805.35 \text { ft bls (driller's depth), whole rock mineralogy in weight } \\
\text { percentage (quartz } 1.7 \text { percent, plagioclase } 0.7 \text { percent, calcite } 23.8 \text { percent, dolomite and } \\
\text { [Fe,Ca]-dolomite } 19.0 \text { percent, total clay minerals } 54.9 \text { percent); clay mineralogy in } \\
\text { weight percentage (illite/smectite* } 8.1 \text { percent, sepiolite } 17.6 \text { percent, palygorskite } 29.2 \\
\text { percent). } * \text { Mixed-layer illite/smectite that contains } 70-80 \text { percent smectite layers } \\
\text { Porosity and permeability: } 1 \text { percent intraparticle porosity, } 0-1 \text { percent fossil moldic } \\
\text { porosity, 0-2 percent vuggy porosity; } 1-4 \text { percent total porosity and low permeability } \\
\text { Depositional environment: Outer ramp } \\
\text { Comments: Base of deepening upward succession at } 808.9 \text { ft bls (driller's depth) } \\
\text { Thin section: G2984-805.35, G2984-807.95 }\end{array}$ \\
\hline $\begin{array}{l}\text { obi } \\
\text { depth: } \\
804.2- \\
811.8 \mathrm{ft} \\
\text { bls } \\
\text { Driller’s } \\
\text { depth: } \\
\text { 808.9- } \\
816.35 \\
\text { ft bls }\end{array}$ & $\begin{array}{l}\text { Lithofacies: Diatom-bearing marl } \\
\text { Depositional texture: Diatom-bearing marl interlaminated and interbedded with smaller } \\
\text { benthic foraminifer and globular planktic foraminifer wackestone and mud- to grain- } \\
\text { dominated packstone } \\
\text { Color: Yellowish gray } 5 Y \text { Y } 1 \\
\text { Sedimentary structures: Thinly laminated to thinly bedded } \\
\text { Trace fossils: Sparse traces, but no taxa identified } \\
\text { Ichnofabrics: Ichnofabric index mainly } 1 \text {-2 } \\
\text { Ichnofacies: Not determined } \\
\text { Carbonate grains: Mainly smaller benthic foraminifera, globular planktic foraminifera, } \\
\text { and silt to very fine sand-sized unidentified skeletal fragments; minor ostracods, echinoid } \\
\text { spines. Foraminifera observed in thin section G2984-815.42 include planktic } \\
\text { foraminifera, smaller benthic foraminifera } \\
\text { Accessory grains: } 1-10 \text { percent silt to very fine sand-sized, angular, well sorted quartz } \\
\text { grains; } 1-2 \text { percent silt to very fine sand-sized black grains (probably mainly } \\
\text { phosphorite); diatoms } \\
\text { XRD mineralogy: At } 812.10 \text { ft bls (driller's depth), whole rock mineralogy in weight } \\
\text { percentage (quartz } 2.5 \text { percent, calcite } 21.3 \text { percent, dolomite and [Fe,Ca]-dolomite } 16.6 \\
\text { percent, total clay minerals } 59.6 \text { percent); clay mineralogy in weight percentage } \\
\text { (illite/smectite* } 6.0 \text { percent, sepiolite } 23.1 \text { percent, palygorskite } 30.5 \text { percent). *Mixed- } \\
\text { layer illite/smectite that contains } 70-80 \text { percent smectite layers } \\
\text { Porosity and permeability: Less than } 1 \text { percent visible porosity (intraparticle) and low } \\
\text { permeability } \\
\text { Depositional environment: Outer ramp }\end{array}$ \\
\hline
\end{tabular}




\begin{tabular}{|c|c|}
\hline & 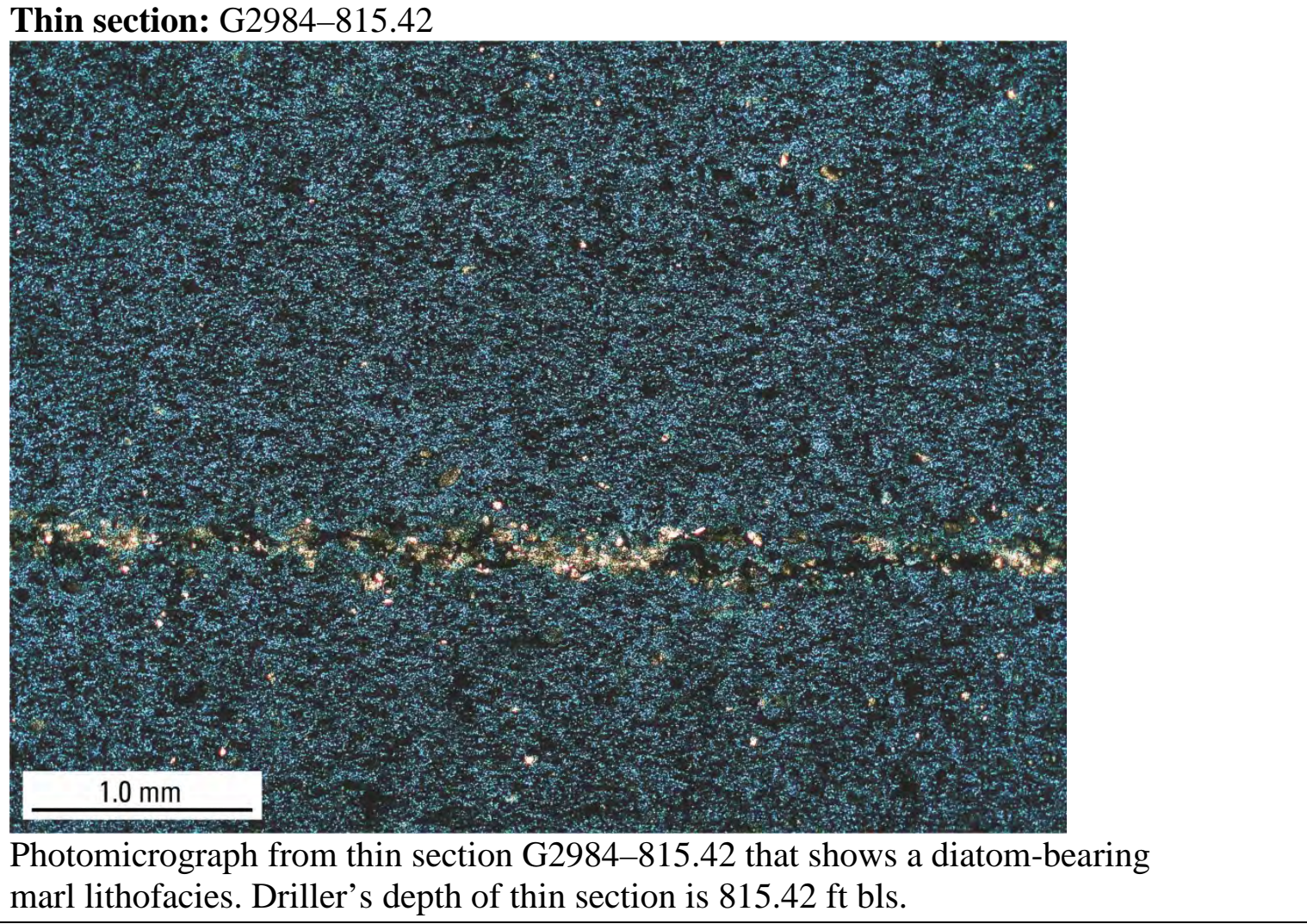 \\
\hline $\begin{array}{l}\text { obi } \\
\text { depth: } \\
\text { 811.8- } \\
820.8 \mathrm{ft} \\
\text { bls } \\
\text { Driller's } \\
\text { depth: } \\
\text { 816.35- } \\
\text { 825.35 } \\
\text { ft bls }\end{array}$ & $\begin{array}{l}\text { Lithofacies: Skeletal and foraminifer wackestone and packstone } \\
\text { Depositional texture: Skeletal, globular planktic and smaller benthic foraminifer } \\
\text { wackestone and mud- to grain-dominated packstone } \\
\text { Color: Yellowish gray 5Y 8/1 } \\
\text { Sedimentary structures: Burrow mottled } \\
\text { Trace fossils: Abundant trace fossils with homogeneous texture throughout } \\
\text { Ichnofabrics: Ichnofabric index } 5 \\
\text { Ichnofacies: Not determined } \\
\text { Carbonate grains: Mainly small fragments of bivalves, globular planktic foraminifera, } \\
\text { smaller benthic foraminifera, and silt to medium sand-sized unidentified skeletal } \\
\text { fragments; minor ostracods, echinoid spines. Foraminifera observed in thin section } \\
\text { G2984-817.57 include planktic foraminifera. Foraminifera observed in thin section } \\
\text { G2984-818.60 include bryozoans, planktic foraminifera, smaller benthic foraminifera. } \\
\text { Foraminifera observed in thin section G2984-824.54 include planktic foraminifera, } \\
\text { smaller benthic foraminifera } \\
\text { Accessory grains: 1-10 percent silt to very fine sand-sized, angular, well sorted quartz } \\
\text { grains; } 1-2 \text { percent silt to very fine sand-sized black grains (probably mainly phosphorite) } \\
\text { Porosity and permeability: Less than } 1 \text { percent visible porosity (interparticle and } \\
\text { intraparticle) and low permeability } \\
\text { Depositional environment: Outer ramp } \\
\text { Comments: Base of deepening upward cycle at } 825.35 \text { ft bls (driller's depth)-lower } \\
\text { interval is burrowed wackestone and packstone part of a deepening upward cycle } \\
\text { Thin section: G2984-817.57, G2984-818.60, G2984-824.54 }\end{array}$ \\
\hline
\end{tabular}




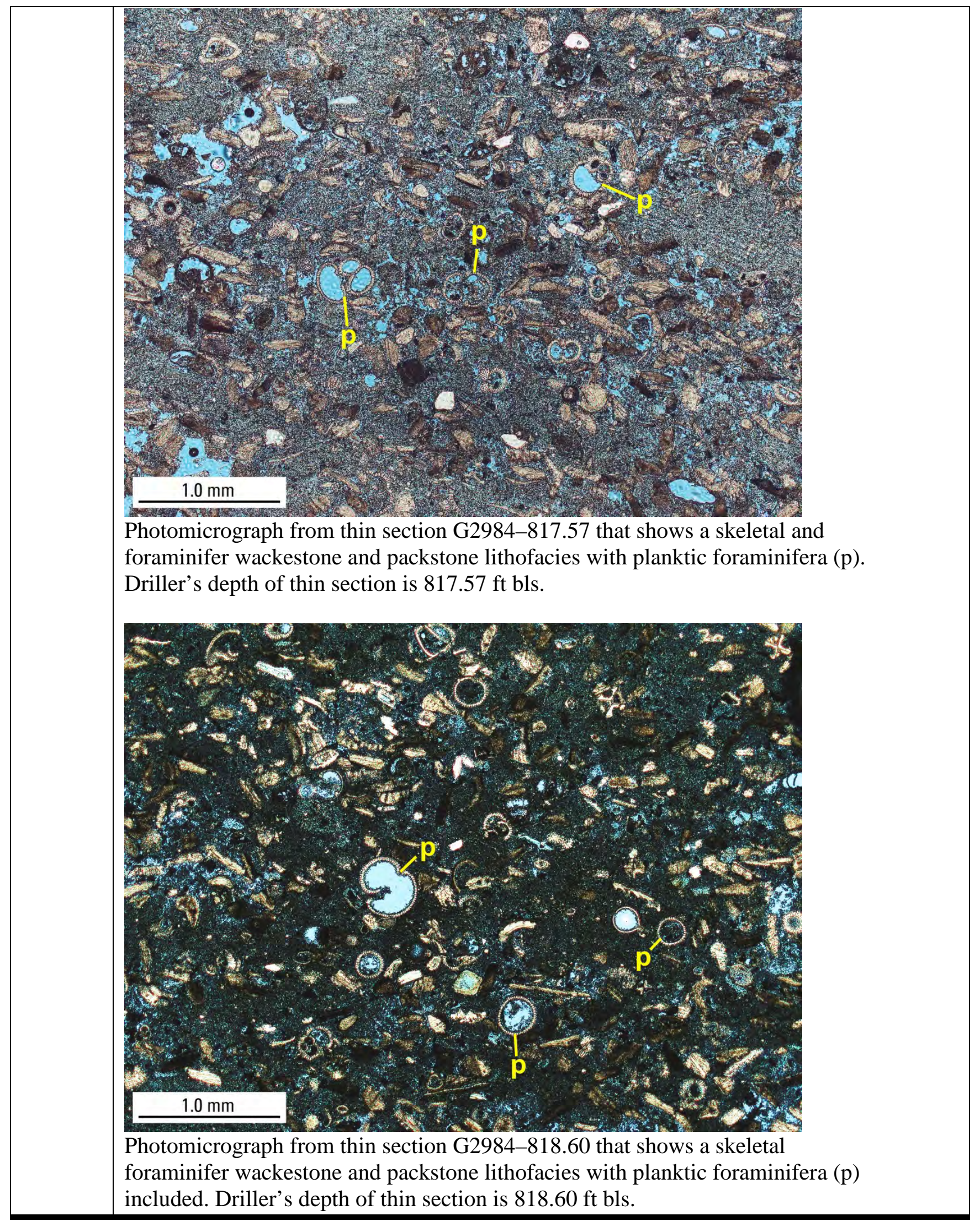




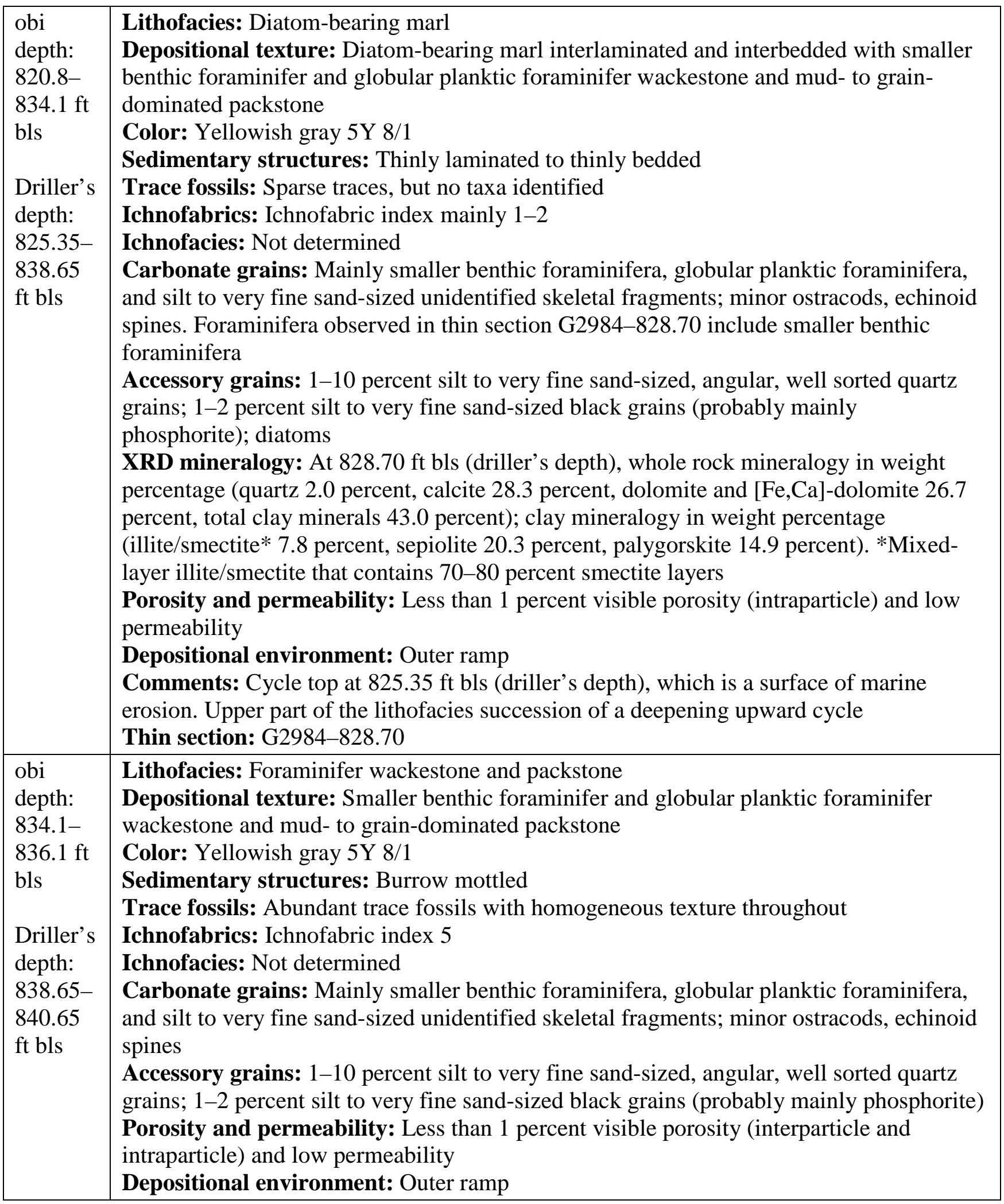




\begin{tabular}{|c|c|}
\hline $\begin{array}{l}\text { obi } \\
\text { depth: } \\
836.1- \\
836.5 \mathrm{ft} \\
\text { bls } \\
\text { Driller's } \\
\text { depth: } \\
\text { 840.65- } \\
\text { 841.05 } \\
\text { ft bls }\end{array}$ & $\begin{array}{l}\text { Lithofacies: Intraclast rudstone } \\
\text { Depositional texture: Intraclast rudstone with a skeletal grainstone matrix } \\
\text { Color: Yellowish gray } 5 Y \text { Y } 8 \\
\text { Sedimentary structures: No burrowing or bedding structures } \\
\text { Carbonate grains: Mainly intraclasts of wackestone, small thin bivalve fragments } \\
\text { composing the matrix, and intraclasts of chert; minor globular planktic foraminifera } \\
\text { Porosity and permeability: } 11 \text { percent interparticle porosity, } 4 \text { percent intraparticle } \\
\text { porosity; } 6 \text { percent total porosity and moderate permeability } \\
\text { Depositional environment: Outer ramp } \\
\text { Comments: Lower part of a deepening upward cycle that is part of a cycle set that } \\
\text { composes depositional sequence Ar6 bounded at its base at } 841.05 \mathrm{ft} \text { bls (driller's depth). } \\
\text { The ideal meter-scale cycle here is (1) intraclast rudstone base, ( } 2 \text { ) about } 1 \text { m thick lower } \\
\text { part of cycle, and (3) overlain by } 1 \text { to several meters of thick laminated mudstone- } \\
\text { deepening upward, fining upward cycle. About } 1 \text { cm of erosional relief on upper bounding } \\
\text { surface of underlying cycle top at } 841.05 \mathrm{ft} \text { bls (driller's depth). Discontinuity is a surface } \\
\text { of marine erosion }\end{array}$ \\
\hline $\begin{array}{l}\text { obi } \\
\text { depth: } \\
836.5- \\
873.5 \mathrm{ft} \\
\text { bls } \\
\text { Driller's } \\
\text { depth: } \\
\text { 841.05- } \\
\text { 878.05 } \\
\text { ft bls }\end{array}$ & 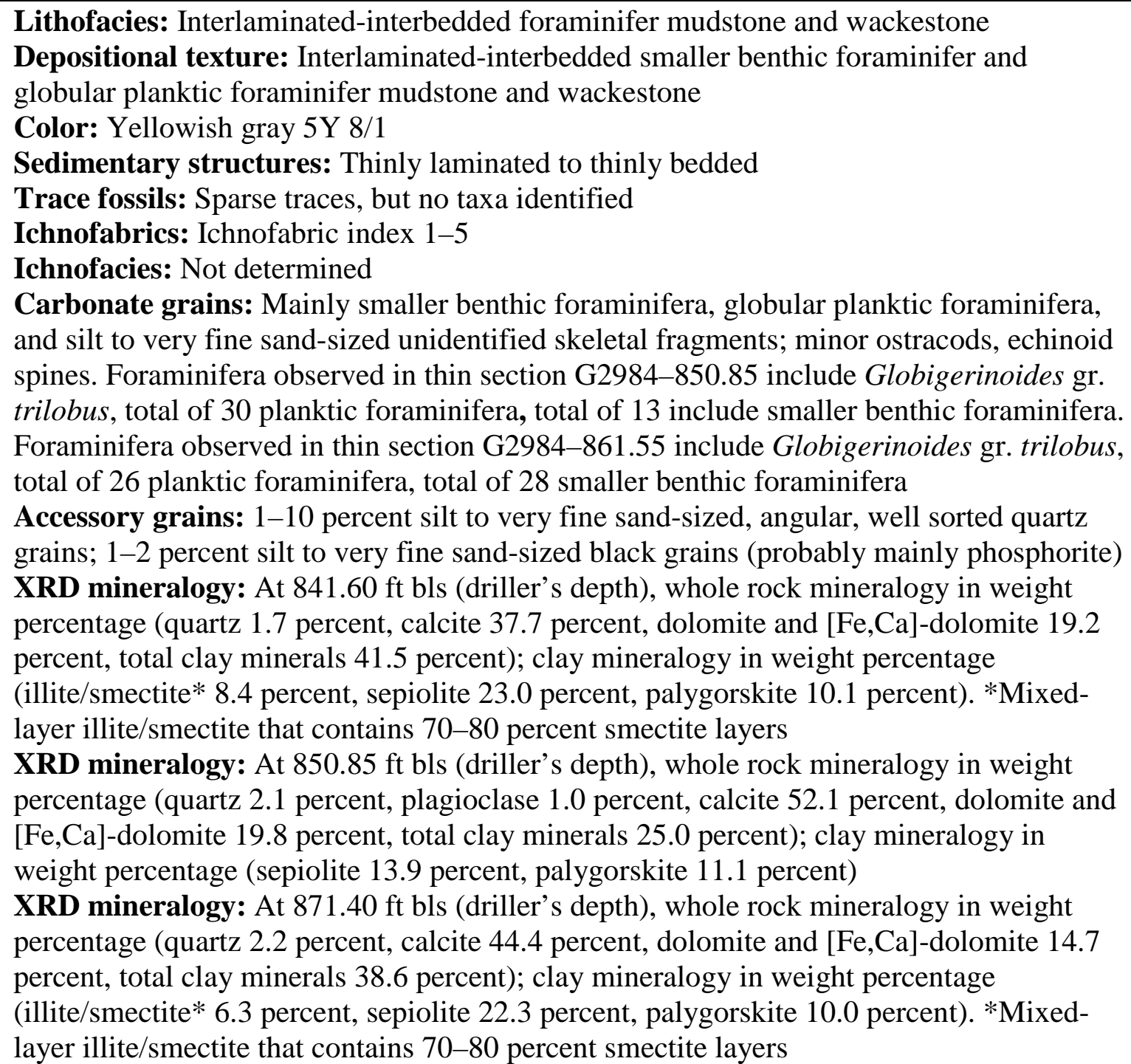 \\
\hline
\end{tabular}




\begin{tabular}{|c|c|}
\hline & $\begin{array}{l}\text { Porosity and permeability: Less than } 1 \text { percent visible porosity (intraparticle) and low } \\
\text { permeability } \\
\text { Depositional environment: Lowstand wedge at ramp margin } \\
\text { Comments: Possible cycle top at } 841.05 \mathrm{ft} \text { bls (driller's depth). About } 1 \mathrm{~cm} \text { of erosional } \\
\text { relief on upper bounding surface of marine erosion at } 841.05 \mathrm{ft} \text { bls (driller's depth). } \\
\text { Thin section: G2984-850.85, G2984-861.40 }\end{array}$ \\
\hline $\begin{array}{l}\text { obi } \\
\text { depth: } \\
873.5- \\
878.2 \mathrm{ft} \\
\text { bls } \\
\\
\text { Driller's } \\
\text { depth: } \\
878.05- \\
882.75 \\
\text { ft bls }\end{array}$ & $\begin{array}{l}\text { Lithofacies: Interlaminated-interbedded foraminifer lime mudstone, wackestone, and } \\
\text { packstone } \\
\text { Depositional texture: Interlaminated-interbedded smaller benthic foraminifer and } \\
\text { globular planktic foraminifer mudstone and wackestone, and mud-dominated packstone } \\
\text { with minor very clay-rich wackestone thick laminations and very thin beds } \\
\text { Color: Yellowish gray } 5 Y \text { Y } 8 \text { 1 } \\
\text { Sedimentary structures: Thinly laminated to thinly bedded } \\
\text { Trace fossils: Sparse traces, but no taxa identified } \\
\text { Ichnofabrics: Ichnofabric index } 1-2 \\
\text { Ichnofacies: Not determined } \\
\text { Carbonate grains: Mainly smaller benthic foraminifera, globular planktic foraminifera, } \\
\text { and silt to very fine sand-sized unidentified skeletal fragments; minor ostracods, echinoid } \\
\text { spines } \\
\text { Accessory grains: } 1-15 \text { percent silt to very fine sand-sized, angular, well sorted quartz } \\
\text { grains; } 1 \text {-2 percent silt to very fine sand-sized black grains (probably mainly } \\
\text { phosphorite); minor very clay-rich thick laminae and very thin beds } \\
\text { Porosity and permeability: Less than } 1 \text { percent visible porosity (intraparticle) and low } \\
\text { permeability } \\
\text { Depositional environment: Lowstand wedge at ramp margin }\end{array}$ \\
\hline $\begin{array}{l}\text { obi } \\
\text { depth: } \\
878.2- \\
881.0 \mathrm{ft} \\
\text { bls } \\
\\
\text { Driller’s } \\
\text { depth: } \\
882.75- \\
885.75 \\
\text { ft bls }\end{array}$ & $\begin{array}{l}\text { Lithofacies: Foraminiferal packstone and grainstone } \\
\text { Depositional texture: Smaller benthic foraminifer and globular planktic foraminifer mud- } \\
\text { and grain-dominated packstone and grainstone } \\
\text { Color: Yellowish gray 5Y 8/1 } \\
\text { Sedimentary structures: Very thickly bedded } \\
\text { Trace fossils: Generally biomottled texture, but no trace fossil taxonomy identified } \\
\text { Ichnofabrics: Ichnofabric index } 1 \text { and } 5 \\
\text { Ichnofacies: None identified } \\
\text { Carbonate grains: Mainly smaller benthic foraminifera, globular planktic foraminifera, } \\
\text { and medium to coarse sand-sized bivalve fragments; minor fine-medium sand-sized } \\
\text { unidentified skeletal fragments, ostracods, echinoid spines } \\
\text { Accessory grains: } 5 \text { percent very fine to fine sand-sized, subangular to angular, well } \\
\text { sorted quartz grains; 5-7 percent fine sand-sized black phosphorite grains } \\
\text { Porosity and permeability: 3-7 percent interparticle porosity, 1-3 percent intraparticle } \\
\text { porosity; } 4 \text {-10 percent total porosity and low permeability } \\
\text { Depositional environments: Lowstand wedge at ramp margin } \\
\text { Comments: A few silicified areas forming chert nodules }\end{array}$ \\
\hline $\begin{array}{l}\text { obi } \\
\text { depth: } \\
881.0- \\
902.85\end{array}$ & $\begin{array}{l}\text { Lithofacies: Interlaminated marl and foraminifer wackestone } \\
\text { Depositional texture: Interlaminated marl and smaller benthic foraminifer and globular } \\
\text { planktic foraminifer wackestone } \\
\text { Color: Yellowish gray 5Y 8/1 }\end{array}$ \\
\hline
\end{tabular}




\begin{tabular}{|c|c|}
\hline $\mathrm{ft}$ bls & $\begin{array}{l}\text { Sedimentary structures: Thinly laminated to thinly bedded to completely burrow } \\
\text { mottled }\end{array}$ \\
\hline $\begin{array}{l}\text { Driller's } \\
\text { depth: } \\
885.75- \\
907.1 \mathrm{ft} \\
\text { bls }\end{array}$ & $\begin{array}{l}\text { Trace fossils: Scarce to abundant unidentified trace fossils, minor Zoophycos } \\
\text { Ichnofabrics: Ichnofabric index 1-5 } \\
\text { Ichnofacies: Cruziana or Zoophycos with a Glossifungites ichnofacies capping the cycle } \\
\text { Carbonate grains: Mainly smaller benthic foraminifera, globular planktic foraminifera, } \\
\text { and silt to very fine sand-sized unidentified skeletal fragments; minor ostracods, echinoid } \\
\text { spines. Foraminifera observed in thin section G2984-898.00 include Globigerinoides gr. } \\
\text { trilobus, total of } 27 \text { planktic foraminifera, total of } 50 \text { smaller benthic foraminifera. } \\
\text { Foraminifera observed in thin section G2984-899.98 include bolivinids (dominant), } \\
\text { rotaliids, planktic foraminifera, smaller benthic foraminifera (dominant). Foraminifera } \\
\text { observed in thin section G2984-903.40 include bolivinids, rotaliids, planktic foraminifera, } \\
\text { smaller benthic foraminifera (dominant) } \\
\text { Accessory grains: } 1-15 \text { percent silt to very fine sand-sized, angular, well sorted quartz } \\
\text { grains; } 1-3 \text { percent silt to very fine sand-sized black grains (probably mainly } \\
\text { phosphorite); common very clay-rich thick laminae and very thin beds } \\
\text { XRD mineralogy: At } 898.00 \text { ft bls (driller's depth), whole rock mineralogy in weight } \\
\text { percentage (quartz } 1.6 \text { percent, calcite } 26.1 \text { percent, dolomite and [Fe,Ca]-dolomite } 14.7 \\
\text { percent, total clay minerals } 57.6 \text { percent); clay mineralogy in weight percentage } \\
\text { (illite/smectite* } 8.2 \text { percent, sepiolite } 23.7 \text { percent, palygorskite } 25.7 \text { percent). } * \text { Mixed- } \\
\text { layer illite/smectite that contains } 70-80 \text { percent smectite layers } \\
\text { Porosity and permeability: Less than } 1 \text { percent visible porosity (intraparticle) and low } \\
\text { permeability } \\
\text { Depositional environment: Outer ramp } \\
\text { Comments: Discontinuity and top of depositional sequence Ar5 at } 885.75 f t \text { bls (driller's } \\
\text { depth) is a surface of marine erosion with Thalassinoides-dominated Glossifungites } \\
\text { ichnofacies developed for about } 5 \text { in. below the upper bounding surface of erosion. Upper } \\
\text { several inches of cycle are a firmground. Major shift in grain size at } 885.75 \text { ft bls (driller's } \\
\text { depth) - coarse overlying fine. Cycle fines upward and grainstones thin upward } \\
\text { Thin section: G2984-898.00, G2984-899.98, G2984-903.40 }\end{array}$ \\
\hline
\end{tabular}




\begin{tabular}{|c|c|}
\hline & 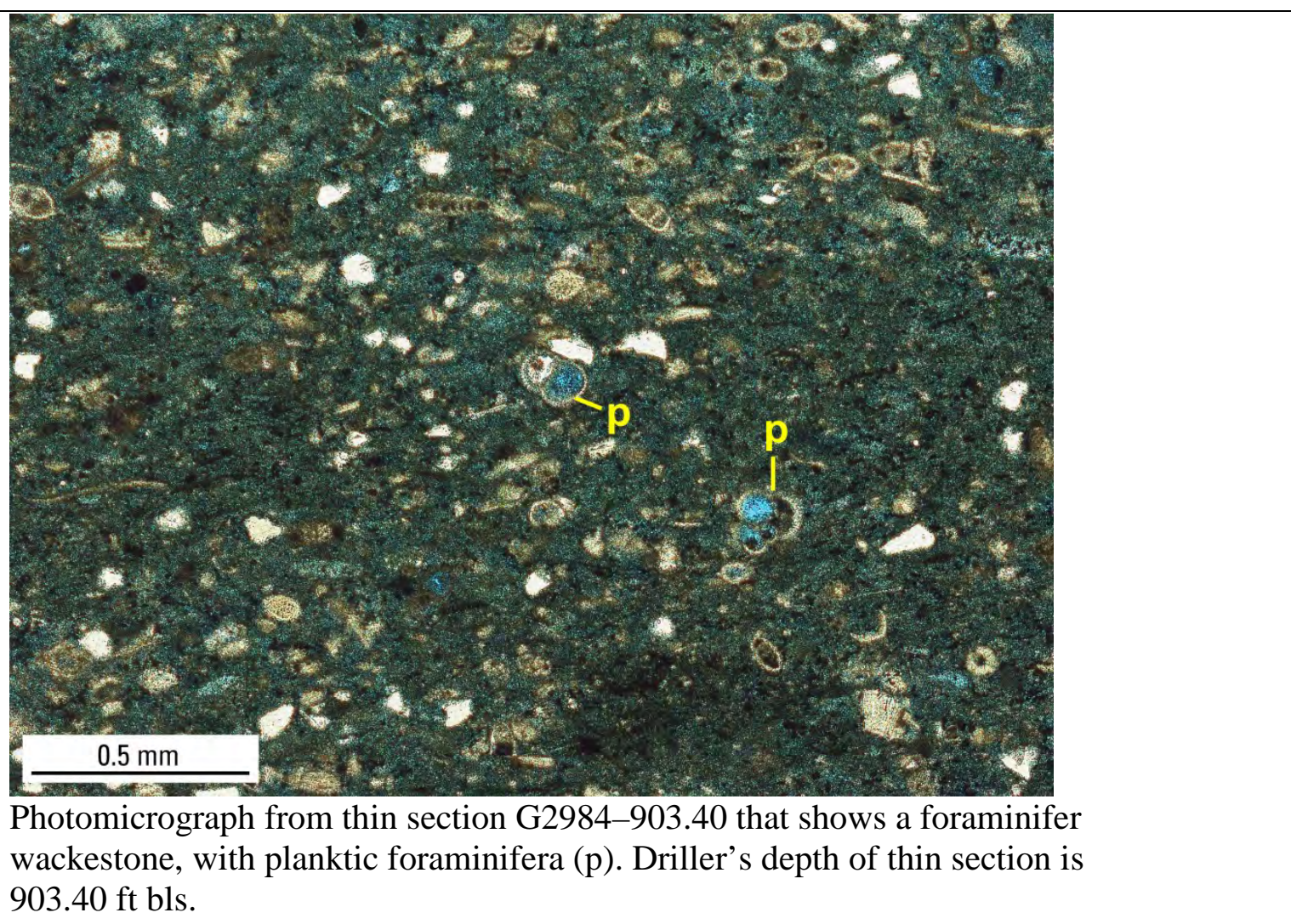 \\
\hline $\begin{array}{l}\text { obi } \\
\text { depth: } \\
902.85- \\
907.0 \mathrm{ft} \\
\text { bls } \\
\text { Driller's } \\
\text { depth: } \\
907.1- \\
910.9 \mathrm{ft} \\
\text { bls }\end{array}$ & $\begin{array}{l}\text { Lithofacies: Terrigenous mudstone } \\
\text { Depositional texture: Terrigenous mudstone } \\
\text { Color: Yellowish gray } 5 Y \text { Y } 8 / 1 \\
\text { Trace fossils: Thalassinoides, one possible Terebellina (Schaubcylindrichnus) at } 907.8 \mathrm{ft} \\
\text { bls 9(driller's depth) } \\
\text { Ichnofabrics: Ichnofabric index } 1-5 \\
\text { Ichnofacies: Cruziana or Zoophycos with a Glossifungites cap } \\
\text { Carbonate grains: Small benthic and globular planktic foraminifera and unidentified } \\
\text { grains } \\
\text { Accessory grains: Very fine sand to medium sand-sized phosphorite grains filling } \\
\text { Thalassinoides burrows at cycle top } \\
\text { Porosity and permeability: Less than } 1 \text { percent visible intraparticle porosity and low } \\
\text { permeability } \\
\text { Depositional environment: Outer ramp } \\
\text { Comments: Major shift in lithology across top of this interval at } 907.1 \mathrm{ft} \text { bls (driller's } \\
\text { depth). Cycle top is at } 908.5 \mathrm{ft} \text { bls (driller's depth). Uppermost } 3 \text { in. is a firmground } \\
\text { characterized by a Thalassinoides-dominated Glossifungites ichnofacies. Uppermost } 3 \text { in. } \\
\text { is silicified as chert-all is part of the upper capping Glossifungites ichnofacies }\end{array}$ \\
\hline $\begin{array}{l}\text { obi } \\
\text { depth: } \\
907.0- \\
920.6 \mathrm{ft} \\
\text { bls }\end{array}$ & $\begin{array}{l}\text { Lithofacies: Terrigenous mudstone } \\
\text { Depositional texture: Terrigenous mudstone } \\
\text { Color: Yellowish gray } 5 Y \text { Y } 8 / 1 \\
\text { Sedimentary structures: Thinly laminated to thinly bedded to completely burrowed } \\
\text { mottled }\end{array}$ \\
\hline
\end{tabular}




\begin{tabular}{|c|c|}
\hline $\begin{array}{l}\text { Driller’s } \\
\text { depth: } \\
\text { 910.9- } \\
924.5 \mathrm{ft} \\
\text { bls }\end{array}$ & 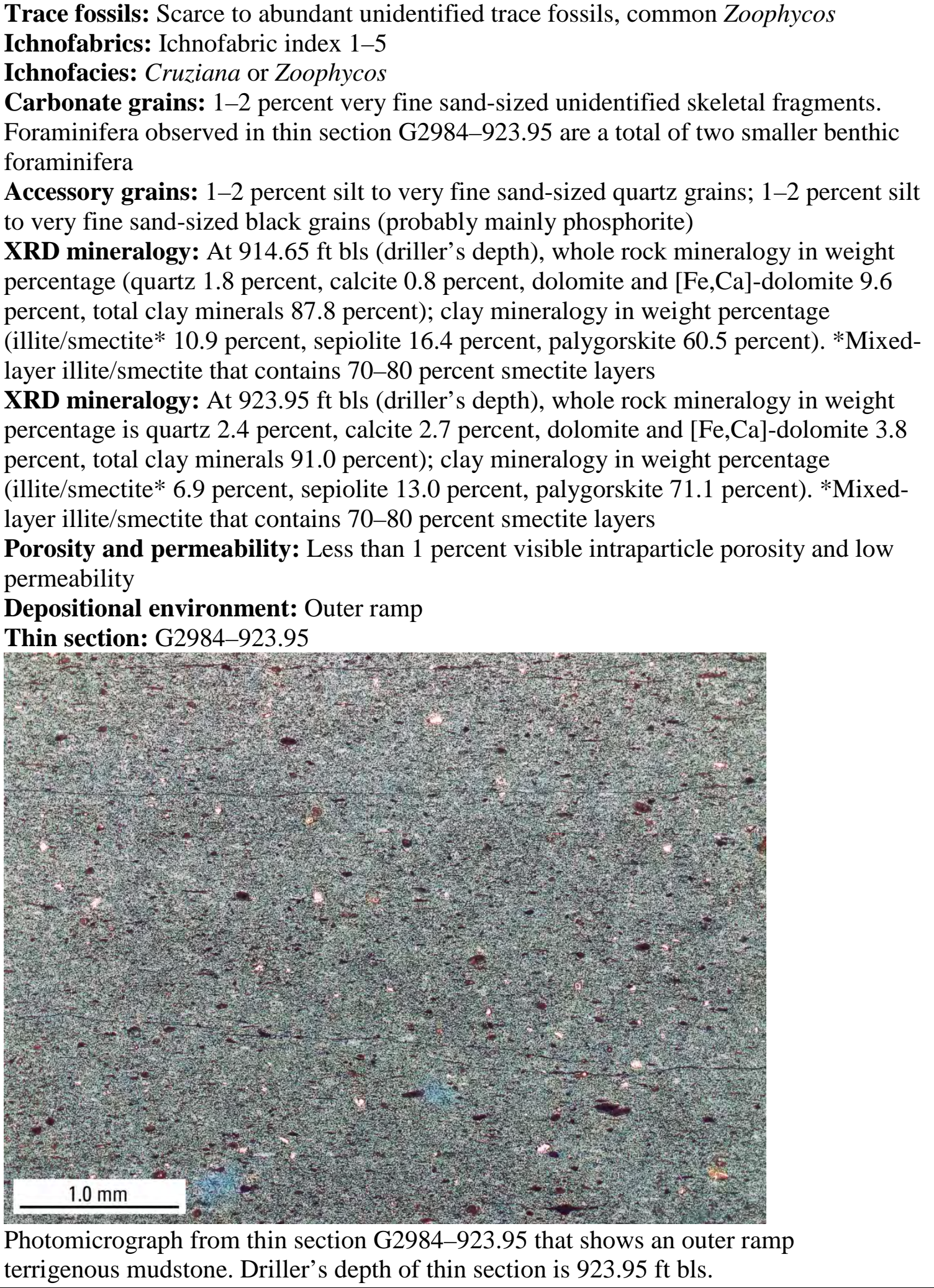 \\
\hline $\begin{array}{l}\text { obi } \\
\text { depth: } \\
\text { 920.6- }\end{array}$ & $\begin{array}{l}\text { Lithofacies: Dolomitic Hyotissa sp. aff. } H \text {. haitensis rudstone } \\
\text { Depositional texture: Dolomitic Hyotissa sp. aff. H. haitensis rudstone with dolomitized } \\
\text { bivalve wackestone matrix }\end{array}$ \\
\hline
\end{tabular}




\begin{tabular}{|c|c|}
\hline $\begin{array}{l}\text { Driller's } \\
\text { depth: } \\
\text { 924.5- } \\
\text { 928.7 ft } \\
\text { bls }\end{array}$ & $\begin{array}{l}\text { Color: Yellowish gray 5Y 7/2 with white N9 Hyotissa sp. aff. H. haitensis } \\
\text { Ichnofabrics: Ichnofabric index 5? } \\
\text { Carbonate grains: Bivalves (commonly disarticulated), benthic and larger foraminifera } \\
\text { (including amphisteginids), echinoid plates and spines. Foraminifera observed in thin } \\
\text { section G2984-924.75 include Globoquadrina sp., Globigerinoides bisphericus?, } \\
\text { Globigerinoides gr. trilobus, miliolids, lenticulinids, rotaliids, planktic foraminifera, } \\
\text { smaller benthic foraminifera. Foraminifera observed in thin section G2984-926.45 } \\
\text { include bryozoans, smaller benthic foraminifera } \\
\text { Accessory grains: 1-2 percent very fine-sized, angular, well-sorted quartz grains; 3-10 } \\
\text { percent fine sand size to small pebble size, angular to well-rounded phosphorite grains } \\
\text { Porosity and permeability: } 2 \text { percent bored porosity, } 1 \text { percent interparticle porosity, } 1 \\
\text { percent intraparticle porosity, } 5 \text { percent vuggy porosity; } 9 \text { percent total porosity and low } \\
\text { permeability } \\
\text { Depositional environment: Low energy lagoon or marine bay } \\
\text { Comments: At the top of composite depositional sequence Ar1 and depositional sequence } \\
\text { Ar4 phosphatization and formation of a hardground is evident along this uppermost part } \\
\text { of the interval at } 924.5 \mathrm{ft} \text { bls (driller's depth), irregular vugs extend downward from the } \\
\text { upper surface for about } 1 \mathrm{ft} \text { with their walls phosphatized, possibly a drowning } \\
\text { unconformity with dissolution along the surface and below, and phosphatization during a } \\
\text { long period of submersion without sediment accumulation; globular planktic foraminifera } \\
\text { within the phosphatized internal fill of the vugs is suggestive of infill during relative } \\
\text { higher sea-level conditions. Drowning unconformity } \\
\text { Thin section: G2984-924.75, G2984-926.45 }\end{array}$ \\
\hline $\begin{array}{l}\text { obi } \\
\text { depth: } \\
924.8- \\
926.1 \mathrm{ft} \\
\text { bls } \\
\\
\text { Driller's } \\
\text { depth: } \\
\text { 928.7- } \\
\text { 930.5 ft } \\
\text { bls }\end{array}$ & $\begin{array}{l}\text { Lithofacies: Sucrosic dolomite } \\
\text { Depositional texture: Sucrosic dolomite } \\
\text { Color: Yellowish gray } 5 Y \text { Y } 72 \\
\text { Sedimentary structures: Burrow mottled throughout interval } \\
\text { Trace fossils: Thalassinoides, Zoophycos?, Planolites? } \\
\text { Ichnofabrics: Ichnofabric index } 5 \\
\text { Ichnofacies: Cruziana? } \\
\text { Carbonate grains: None determined } \\
\text { Accessory grains: } 40 \text { percent sucrosic dolomite } \\
\text { Porosity and permeability: } 3 \text { percent vuggy porosity; } 3 \text { percent total porosity and low } \\
\text { permeability } \\
\text { Depositional environment: Low-energy restricted lagoon or marine bay on inner shelf, } \\
\text { subtidal to intertidal }\end{array}$ \\
\hline $\begin{array}{l}\text { Driller’s } \\
\text { depth: } \\
930.5- \\
938.0 \mathrm{ft}\end{array}$ & $\begin{array}{l}\text { Lithofacies: Dolomitic Hyotissa sp. aff. H. haitensis rudstone } \\
\text { Depositional texture: Dolomitic Hyotissa sp. aff. H. haitensis rudstone with dolomitic } \\
\text { bivalve wackestone matrix } \\
\text { Color: Yellowish gray } 5 Y \text { Y 7/2 with white N9 Hyotissa sp. aff. H. haitensis } \\
\text { Ichnofabrics: Ichnofabric index 5? } \\
\text { Carbonate grains: Bivalves (commonly disarticulated), benthic and larger foraminifera } \\
\text { (including amphisteginids), echinoid plates and spines. Foraminifera observed in thin } \\
\text { section G2984-936.00 total of two Nummulites panamensis. } \\
\text { Accessory grains: } 1-2 \text { percent medium sand to coarse-sized (mostly coarse sand size), } \\
\text { subrounded, moderately sorted quartz grains; } 3-7 \text { percent fine sand size to small pebble }\end{array}$ \\
\hline
\end{tabular}




\begin{tabular}{|l|l|}
\hline bls & $\begin{array}{l}\text { size, angular to well-rounded phosphorite grains } \\
\text { Porosity and permeability: 2 percent bored porosity, } 1 \text { percent interparticle porosity, } 1 \\
\text { percent intraparticle porosity, 5 percent vuggy porosity; 9 percent total porosity and low } \\
\text { permeability } \\
\text { Depositional environment: Low-energy lagoon or marine bay on inner shelf, shallow } \\
\text { subtidal } \\
\text { Thin section: G2984-936.00 }\end{array}$ \\
& $\begin{array}{l}\text { Photograph from core that shows Hyotissa sp. aff. H. haitensis from a driller's } \\
\text { depth of } 935.3 \mathrm{ft} \text { bls. Photograph by G. Lynn Wingard, U.S. Geological Survey. }\end{array}$
\end{tabular}




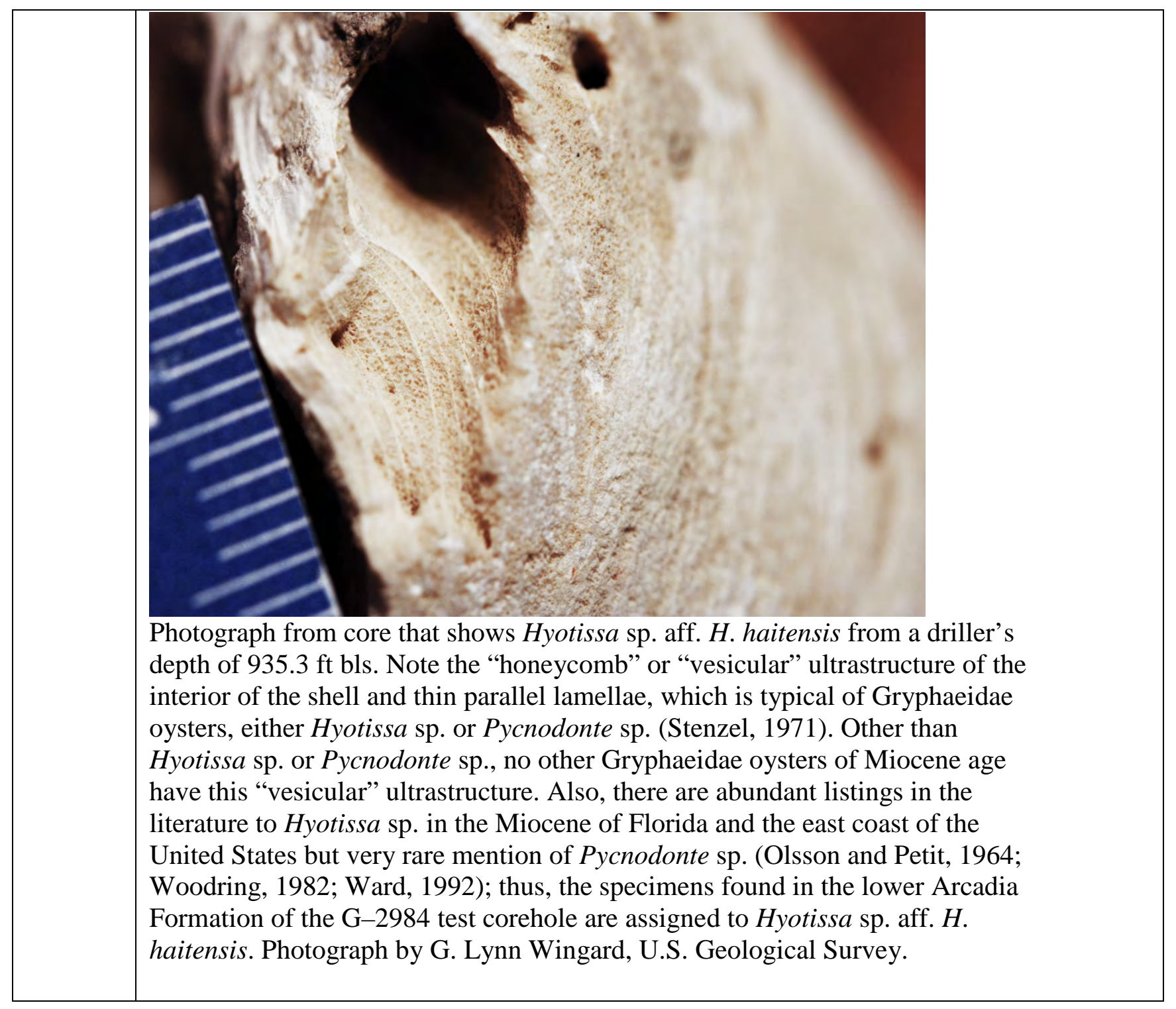




\begin{tabular}{|c|c|}
\hline & 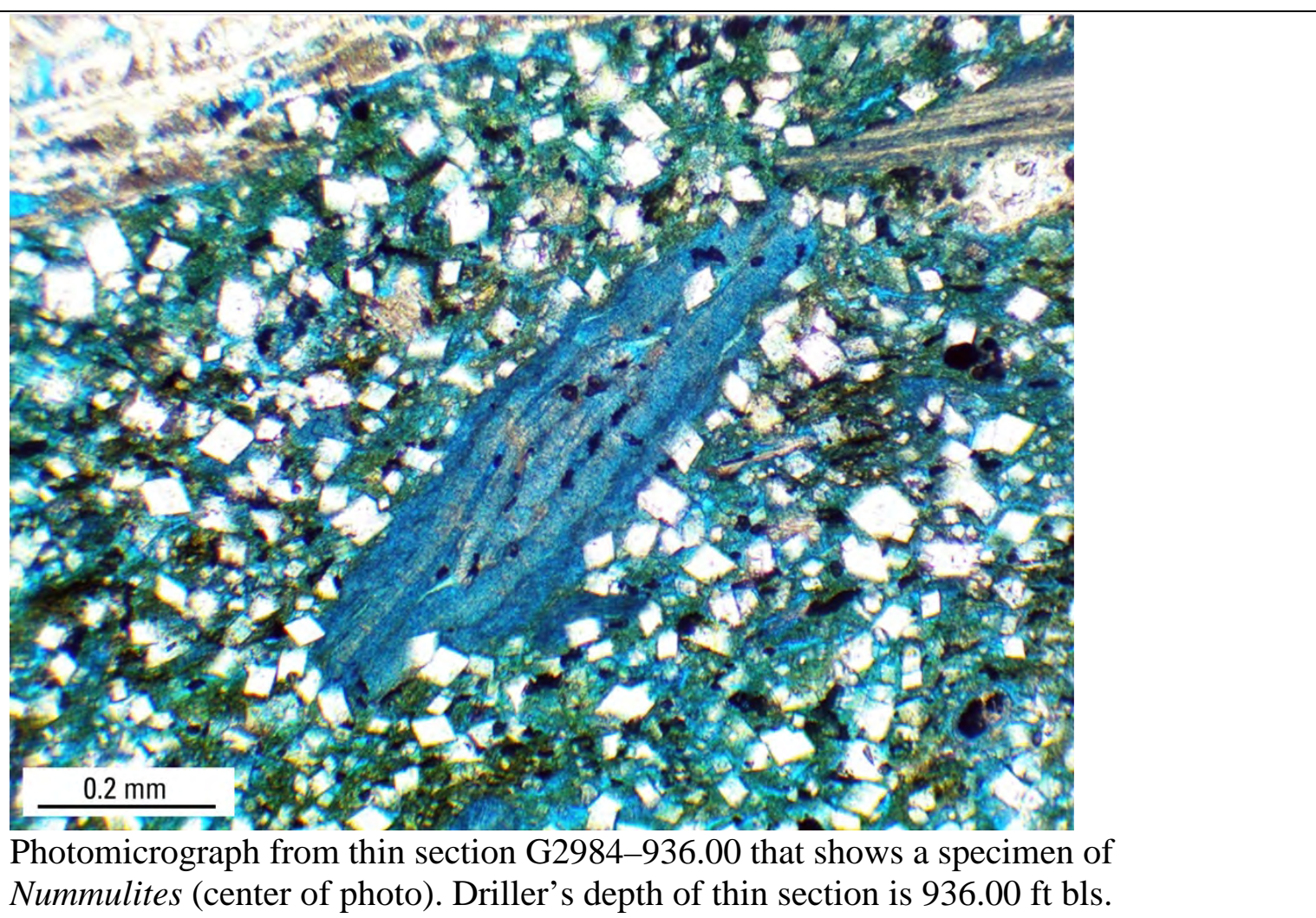 \\
\hline $\begin{array}{l}\text { obi } \\
\text { depth: } \\
\text { 934-939 } \\
\text { ft bls } \\
\\
\text { Driller's } \\
\text { depth: } \\
\text { 938- } \\
\text { 943.2 ft } \\
\text { bls }\end{array}$ & $\begin{array}{l}\text { Lithofacies: Sandy, phosphatic skeletal packstone } \\
\text { Depositional texture: Sandy, phosphatic, bivalve floatstone with skeletal mud-dominated } \\
\text { packstone matrix } \\
\text { Color: Yellowish gray 5Y 8/1 } \\
\text { Sedimentary structures: Burrow mottled throughout interval } \\
\text { Ichnofabrics: Ichnofabric index } 5 \\
\text { Carbonate grains: Mainly bivalves (commonly disarticulated) and unidentified skeletal } \\
\text { fragments, smaller and larger benthic foraminifera. Foraminifera observed in thin section } \\
\text { G2984-942.10 include Amphistegina sp., total of two Nummulites panamensis, total of } 10 \\
\text { Miogypsina gr. gunteri, smaller benthic foraminifera } \\
\text { Accessory grains: } 15-20 \text { percent very fine to medium sand-sized (mostly very fine sand } \\
\text { size), angular to subrounded (mainly angular), well-sorted quartz grains; } 10-20 \text { percent } \\
\text { very fine to fine sand size, well-rounded phosphorite grains; } 1 \text { percent plagioclase } \\
\text { Porosity and permeability: } 2 \text { percent moldic porosity, } 1 \text { percent interparticle porosity, } 1 \\
\text { percent intraparticle porosity; } 4 \text { percent total porosity and low permeability } \\
\text { Depositional environment: Marine offshore or lower shoreface to upper shoreface at } \\
\text { uppermost part of cycle } \\
\text { Comments: Upper phosphatized surface is at top of depositional sequence Ar3 at } 938 \mathrm{ft} \\
\text { bls (driller's depth). Karstic dissolution with downward solution piping from upper } \\
\text { surface. Dissolution cavities and upper surface encrusted with black phosphorite. } \\
\text { Drowning unconformity } \\
\text { Thin section: G2984-942.10 }\end{array}$ \\
\hline $\begin{array}{l}\text { obi } \\
\text { depth: }\end{array}$ & $\begin{array}{l}\text { Lithofacies: Sandy, phosphatic skeletal packstone } \\
\text { Depositional texture: Sandy, phosphatic, bivalve floatstone with skeletal mud-dominated }\end{array}$ \\
\hline
\end{tabular}




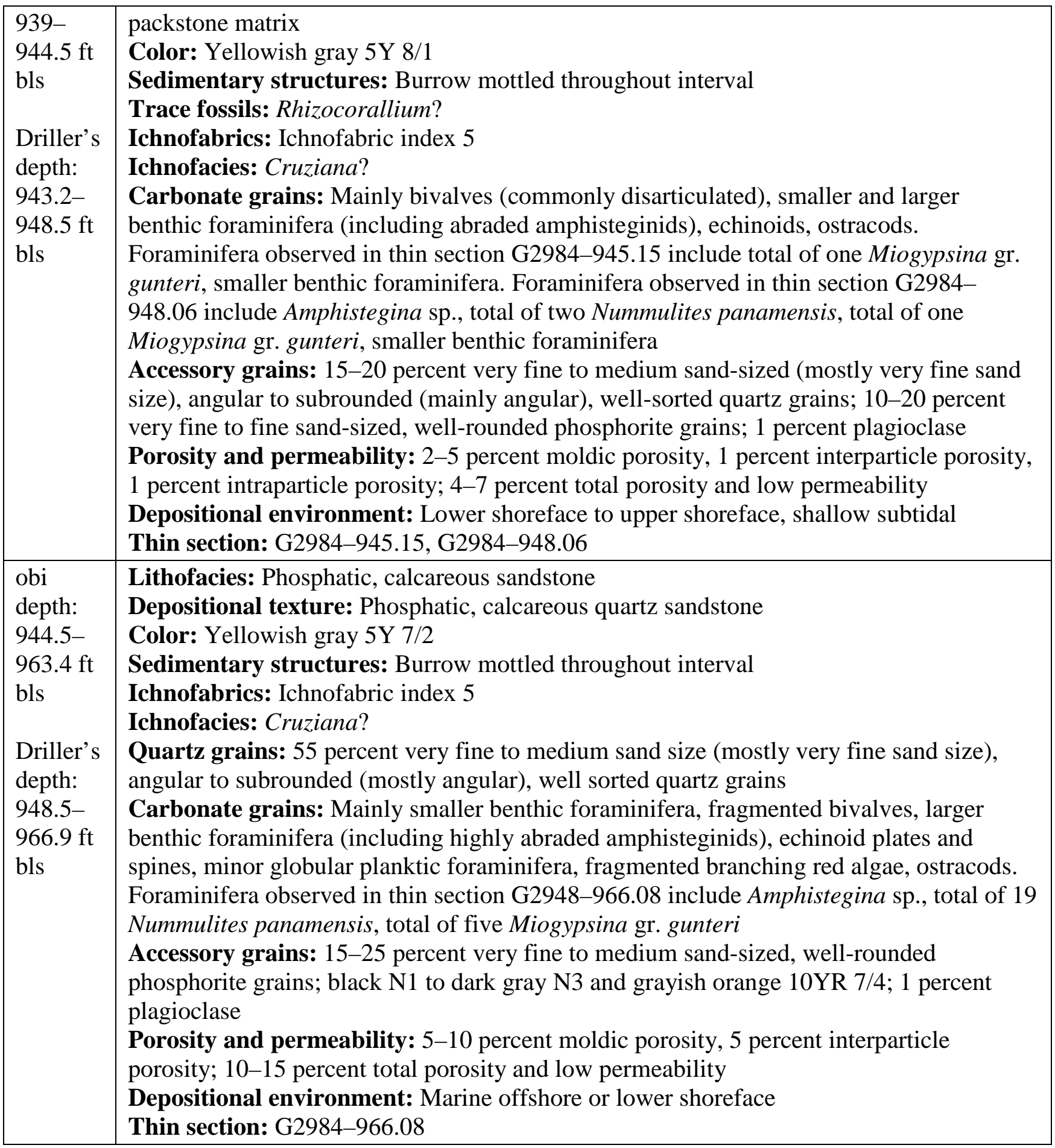




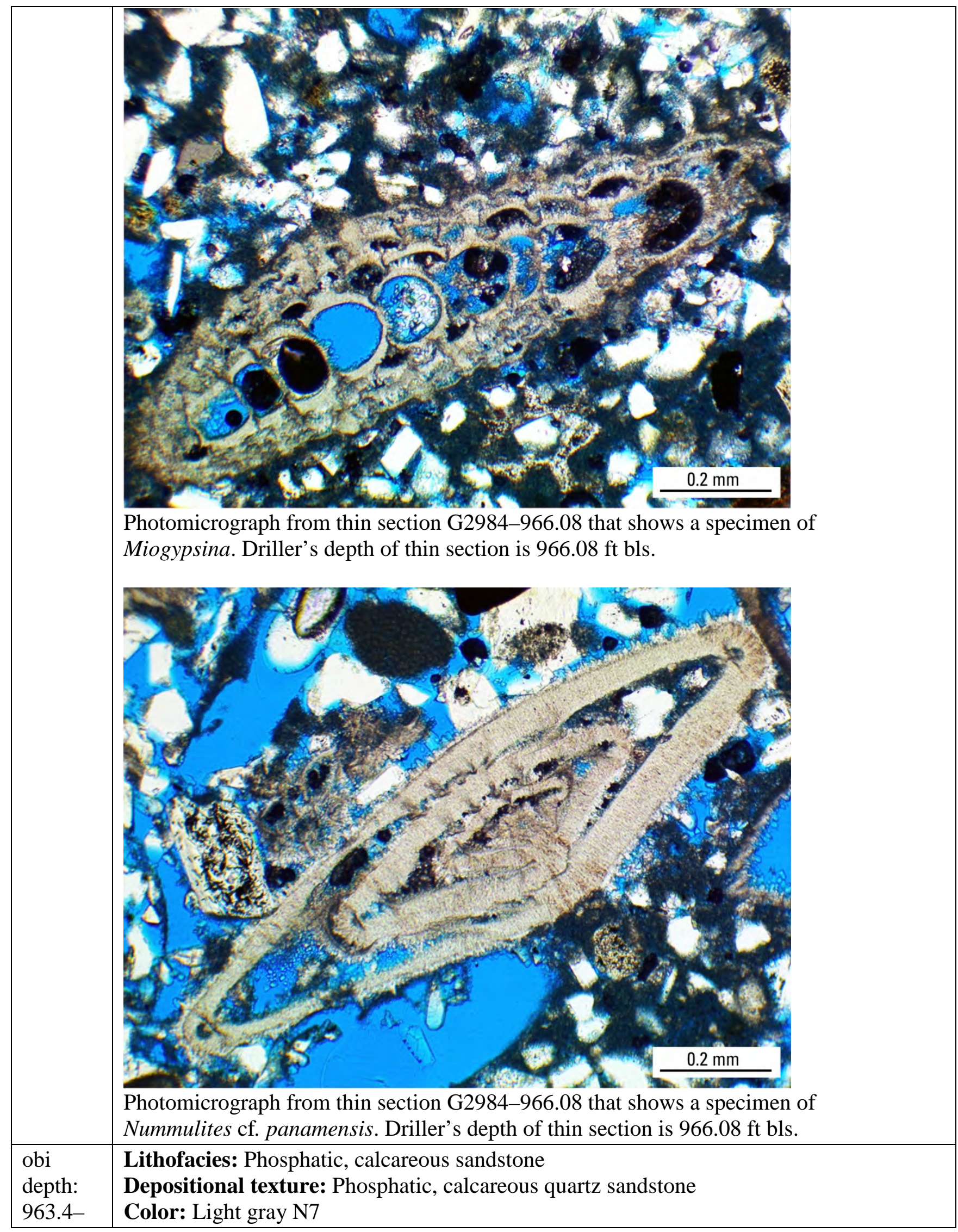




\begin{tabular}{|c|c|}
\hline $\begin{array}{l}\text { 968.7 ft } \\
\text { bls } \\
\text { Driller's } \\
\text { depth: } \\
966.9- \\
973 \mathrm{ft} \\
\text { bls }\end{array}$ & $\begin{array}{l}\text { Sedimentary structures: Burrow mottled throughout interval } \\
\text { Trace fossils: Rhizocorallium? } \\
\text { Ichnofabrics: Ichnofabric index } 5 \\
\text { Ichnofacies: Cruziana? } \\
\text { Quartz grains: } 55 \text { percent very fine to medium sand size (mostly very fine sand size), } \\
\text { angular to subrounded (mostly angular), well sorted quartz grains } \\
\text { Carbonate grains: Mainly fragmented bivalves, larger benthic foraminifera (including } \\
\text { nummulitids, amphisteginids), echinoid plates and spines, minor globular planktic } \\
\text { foraminifera. } \\
\text { Accessory grains: } 15-25 \text { percent very fine to fine sand-sized, well-rounded phosphorite } \\
\text { grains; black N1 to dark gray N3 and grayish orange } 10 \text { YR } 7 / 4 \text {; } 1 \text { percent plagioclase } \\
\text { Porosity and permeability: } 5-10 \text { percent moldic porosity, } 5 \text { percent interparticle } \\
\text { porosity; } 10-15 \text { percent total porosity and low permeability } \\
\text { Depositional environment: Marine offshore or lower shoreface } \\
\text { Comments: Coarsens upward with a centimeter-scale thick red algal? floatstone cap }\end{array}$ \\
\hline $\begin{array}{l}\text { obi } \\
\text { depth: } \\
968.7- \\
972.7 \mathrm{ft} \\
\text { bls } \\
\\
\text { Driller's } \\
\text { depth: } \\
973- \\
977.0 \mathrm{ft} \\
\text { bls }\end{array}$ & $\begin{array}{l}\text { Lithofacies: Phosphatic, calcareous sand } \\
\text { Depositional texture: Phosphatic, skeletal fragment quartz sand } \\
\text { Color: Light gray N7 } \\
\text { Sedimentary structures: Burrow mottled throughout interval } \\
\text { Ichnofabrics: Ichnofabric index } 5 \\
\text { Quartz grains: } 65 \text { percent very fine to fine sand size (mostly very fine sand size), angular } \\
\text { to subrounded (mostly angular), well sorted quartz grains } \\
\text { Carbonate grains: Mainly small unidentified skeletal fragments, fragmented bivalves, } \\
\text { smaller benthic foraminifera } \\
\text { Accessory grains: } 15-25 \text { percent very fine to fine sand-sized, well-rounded phosphorite } \\
\text { grains; black N1 to dark gray N3 and grayish orange } 10 \text { YR } 7 / 4 \text {; } 1 \text { percent plagioclase } \\
\text { Porosity and permeability: } 25 \text { percent interparticle porosity; } 25 \text { percent total porosity } \\
\text { and low permeability } \\
\text { Depositional environment: Possibly lower shoreface to upper shoreface or marine } \\
\text { offshore } \\
\text { Comments: Slightly consolidated sand grains }\end{array}$ \\
\hline $\begin{array}{l}\text { obi } \\
\text { depth: } \\
972.7- \\
983.0 \mathrm{ft} \\
\text { bls } \\
\\
\text { Driller's } \\
\text { depth: } \\
\text { 977.0- } \\
983.0 \mathrm{ft} \\
\text { bls }\end{array}$ & No recovery \\
\hline $\begin{array}{l}\text { obi } \\
\text { depth: } \\
983.0- \\
984.3 \mathrm{ft}\end{array}$ & $\begin{array}{l}\text { Lithofacies: Phosphatic, calcareous sandstone } \\
\text { Depositional texture: Phosphatic, skeletal fragment quartz sandstone } \\
\text { Color: Grades from dark yellowish brown 10YR 4/2 to medium light gray N6 quartz } \\
\text { sandstone and very pale orange 10YR } 8 / 2 \text { carbonate grains and trace fossil walls }\end{array}$ \\
\hline
\end{tabular}




\begin{tabular}{|c|c|}
\hline $\begin{array}{l}\text { Driller's } \\
\text { depth: } \\
983.0- \\
984.3 \mathrm{ft} \\
\text { bls }\end{array}$ & $\begin{array}{l}\text { Sedimentary structures: Burrow mottled throughout interval } \\
\text { Trace fossils: Common Ophiomorpha } \\
\text { Ichnofabrics: Ichnofabric index } 5 \\
\text { Ichnofacies: Distal Skolithos? } \\
\text { Quartz grains: } 40 \text { percent very fine to medium sand size (mostly fine sand size), angular } \\
\text { to subrounded, moderately sorted quartz grains } \\
\text { Carbonate grains: Mainly bivalve fragments, echinoid plates, benthic foraminifera } \\
\text { (including nummulitids and amphisteginid? larger benthic foraminifera. Foraminifera } \\
\text { observed in thin section G2984-984.23 include Amphistegina sp. } \\
\text { Accessory grains: } 25-35 \text { percent very fine to medium sand-sized (mostly fine sand-sized } \\
\text { grains), well-rounded phosphorite grains; black N1 to dark gray N3 and grayish orange } \\
\text { 10YR 7/4 } \\
\text { Porosity and permeability: } 1 \text { percent interparticle porosity, } 1 \text { percent intraparticle } \\
\text { porosity, 1-5 percent moldic porosity; 4-7 percent total porosity and low permeability } \\
\text { Depositional environment: Possibly lower shoreface to upper shoreface, shallow } \\
\text { subtidal } \\
\text { Comments: Coarsens upward } \\
\text { Thin section: G2984-984.23 }\end{array}$ \\
\hline $\begin{array}{l}\text { obi } \\
\text { depth: } \\
984.3- \\
986.3 \mathrm{ft} \\
\text { bls } \\
\\
\text { Driller's } \\
\text { depth: } \\
984.3- \\
986.3 \mathrm{ft} \\
\text { bls }\end{array}$ & $\begin{array}{l}\text { Lithofacies: Phosphatic, calcareous sand } \\
\text { Depositional texture: Phosphatic, skeletal fragment quartz sand } \\
\text { Color: Dark yellowish brown } 10 \text { YR } 4 / 2 \\
\text { Trace fossils: Burrow mottled throughout interval } \\
\text { Ichnofabrics: Ichnofabric index } 5 \\
\text { Quartz grains: } 40 \text { percent very fine to fine sand size, angular to subrounded, well sorted } \\
\text { quartz grains } \\
\text { Carbonate grains: Mainly fragmented bivalve fragments, abraded echinoid plates, highly } \\
\text { abraded benthic foraminifera } \\
\text { Accessory grains: } 25-35 \text { percent very fine to fine sand-sized, well-rounded phosphorite } \\
\text { grains; black N1 to dark gray N3 and grayish orange } 10 \text { YR } 7 / 4 ; 1 \text { percent plagioclase } \\
\text { Porosity and permeability: } 10-30 \text { percent interparticle porosity, } 1 \text { percent intraparticle } \\
\text { porosity, } 1-10 \text { percent moldic porosity; } 12-32 \text { percent total porosity and relatively low to } \\
\text { moderate permeability } \\
\text { Depositional environment: Marine offshore or lower shoreface } \\
\text { Comments: Mostly slightly consolidated or entirely unconsolidated sand grains }\end{array}$ \\
\hline $\begin{array}{l}\text { obi } \\
\text { depth: } \\
986.3- \\
992.0 \mathrm{ft} \\
\text { bls }\end{array}$ & $\begin{array}{l}\text { Lithofacies: Phosphatic, calcareous sandstone } \\
\text { Depositional texture: Phosphatic, skeletal fragment quartz sandstone } \\
\text { Color: Grades from dark yellowish brown 10YR 4/2 to medium light gray N6 } \\
\text { Sedimentary structures: Burrow mottled throughout interval } \\
\text { Trace fossils: Common Ophiomorpha nodosa, minor local Phycosiphon } \\
\text { Ichnofabrics: Ichnofabric index } 5 \\
\text { Ichnofacies: Distal Skolithos } \\
\text { Quartz grains: } 40 \text { percent quartz grains, very fine to medium sand size (mostly fine sand } \\
\text { size), angular to subrounded, moderately sorted } \\
\text { Carbonate grains: Mainly bivalve fragments, echinoid plates and spines, benthic } \\
\text { foraminifera (including nummulitid and amphisteginid larger benthic foraminifera), }\end{array}$ \\
\hline
\end{tabular}




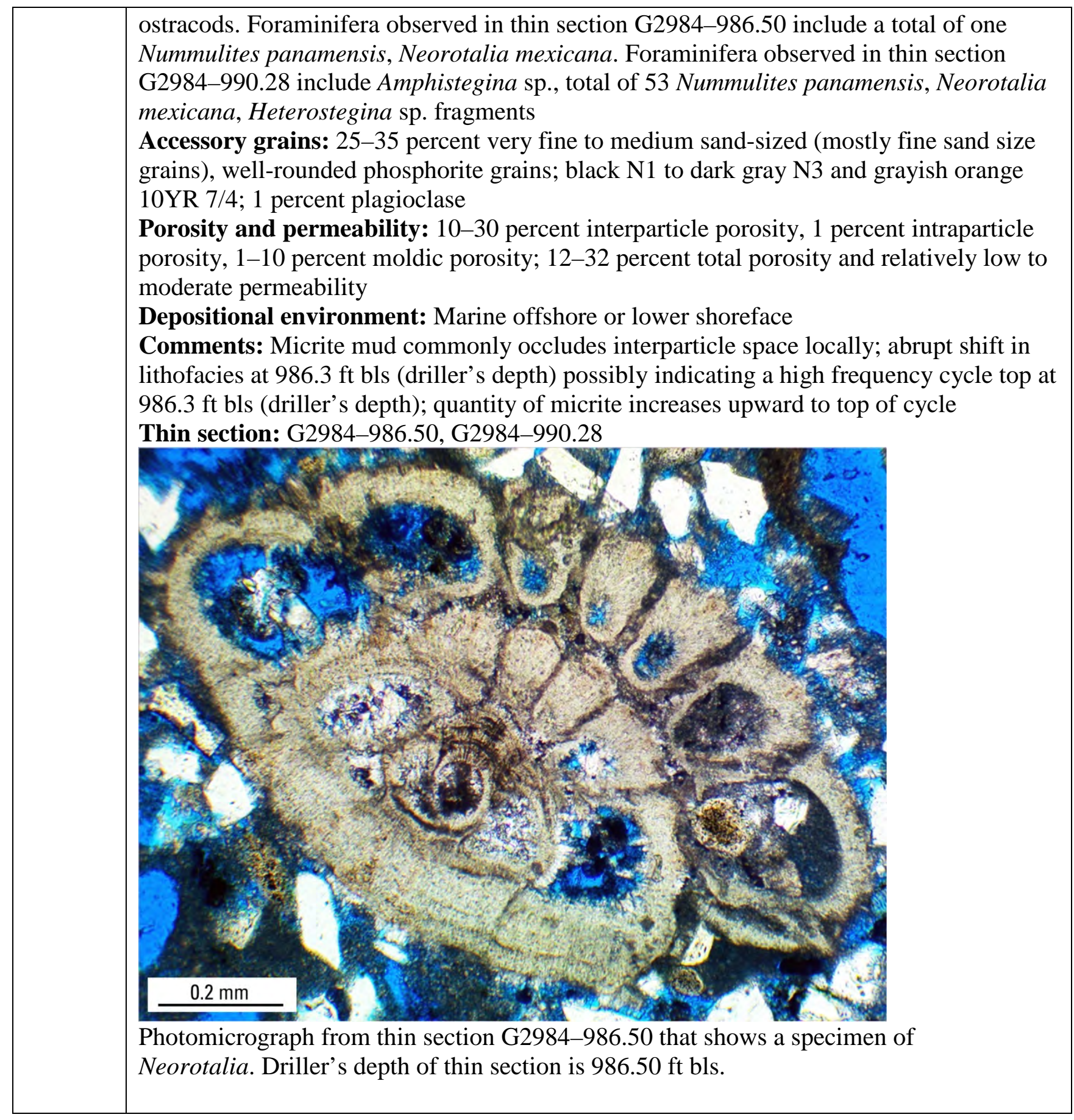




\begin{tabular}{|l|l|l|}
\hline & & \\
\end{tabular}




\begin{tabular}{|c|c|}
\hline & $\begin{array}{l}\text { Neorotalia mexicana? } \\
\text { Accessory grains: } 25-35 \text { percent very fine to medium sand-sized (mostly fine sand-sized } \\
\text { grains), well-rounded phosphorite grains; black N1 to dark gray N3 and grayish orange } \\
\text { 10YR 7/4; } 1 \text { percent plagioclase } \\
\text { Porosity and permeability: } 10-20 \text { percent interparticle porosity, } 1 \text { percent intraparticle } \\
\text { porosity, } 1 \text {-10 percent moldic porosity; } 12-31 \text { percent total porosity and relatively low to } \\
\text { moderate permeability } \\
\text { Depositional environment: Marine offshore or lower shoreface } \\
\text { Comments: Micrite mud commonly occludes interparticle space locally } \\
\text { Thin section: G2984-999.23 }\end{array}$ \\
\hline $\begin{array}{l}\text { obi } \\
\text { depth: } \\
1,003.8- \\
1,019.6 \\
\text { ft bls } \\
\text { Driller's } \\
\text { depth: } \\
1,003.8- \\
1,019.6 \\
\text { ft bls }\end{array}$ & $\begin{array}{l}\text { Lithofacies: Bivalve rudstone } \\
\text { Depositional texture: Bivalve rudstone and floatstone with sandy skeletal wackestone to } \\
\text { skeletal mud- and grain-dominated packstone matrix and sandy skeletal grainstone matrix } \\
\text { in uppermost part of interval } \\
\text { Color: Very light gray N8 } \\
\text { Sedimentary structures: Burrow mottled throughout interval } \\
\text { Trace fossils: Not determined } \\
\text { Ichnofabrics: Ichnofabric index } 4-5 \\
\text { Ichnofacies: Not determined } \\
\text { Carbonate grains: Mainly large gastropods and bivalves (commonly disarticulated), } \\
\text { benthic forams (common rotaliids), echinoids, fragmented branching red algae in } \\
\text { uppermost part of interval. Foraminifera observed in thin section G2984-1003.53 include } \\
\text { Amphistegina sp., total of one Nummulites panamensis, Neorotalia Mexicana. } \\
\text { Foraminifera observed in thin section G2984-1015.90 include Amphistegina sp., } \\
\text { Neorotalia mexicana, smaller benthic foraminifera } \\
\text { Accessory grains: } 15-20 \text { percent matrix of very fine to fine sand-sized, angular to } \\
\text { subrounded, well-sorted quartz grains; } 1-4 \text { percent very fine to fine sand-sized, well- } \\
\text { rounded phosphorite grains; } 1 \text { percent plagioclase } \\
\text { Porosity and permeability: } 5 \text {-18 percent moldic porosity, } 1 \text { percent interparticle } \\
\text { porosity, } 1 \text { percent intraparticle porosity; } 7-20 \text { percent total porosity and relatively low to } \\
\text { moderate permeability } \\
\text { Depositional environment: Marine offshore or lower shoreface to upper shoreface at } \\
\text { uppermost part of depositional sequence Ar2 } \\
\text { Comments: Top of depositional sequence Ar2 at } 1,003.8 \text { ft bls (driller's depth). Sediment } \\
\text { from base of cycle above penetrates downward through dissolutional pore system of Ar2 } \\
\text { cycle a distance of about } 1.3 \mathrm{ft} \text { from the top of depositional sequence Ar2. Interval } \\
\text { becomes more grain rich toward the top, indicating increasing environmental energy } \\
\text { conditions upward or shoaling upward } \\
\text { Thin section: G2984-1003.53, G2984-1015.90 }\end{array}$ \\
\hline
\end{tabular}




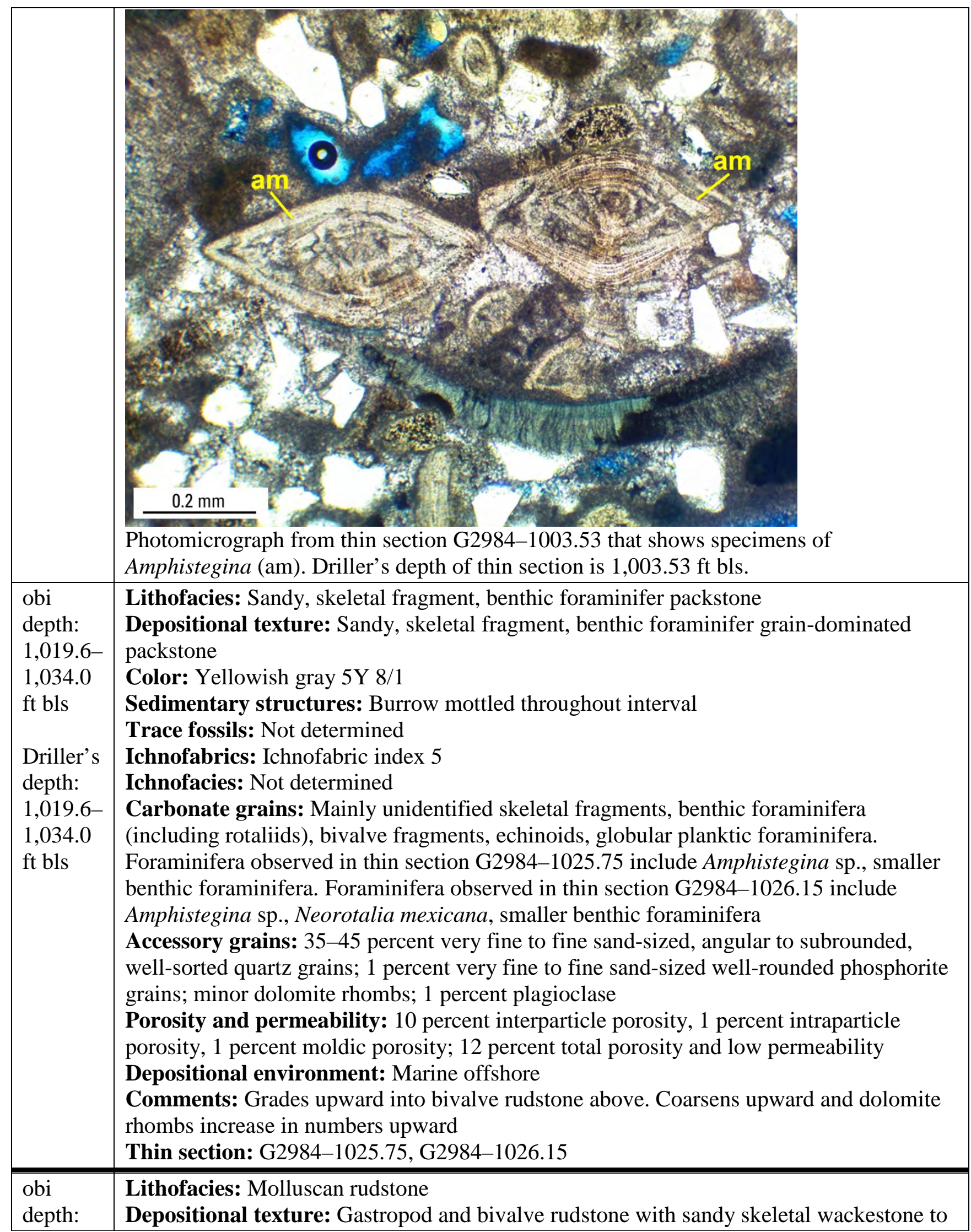




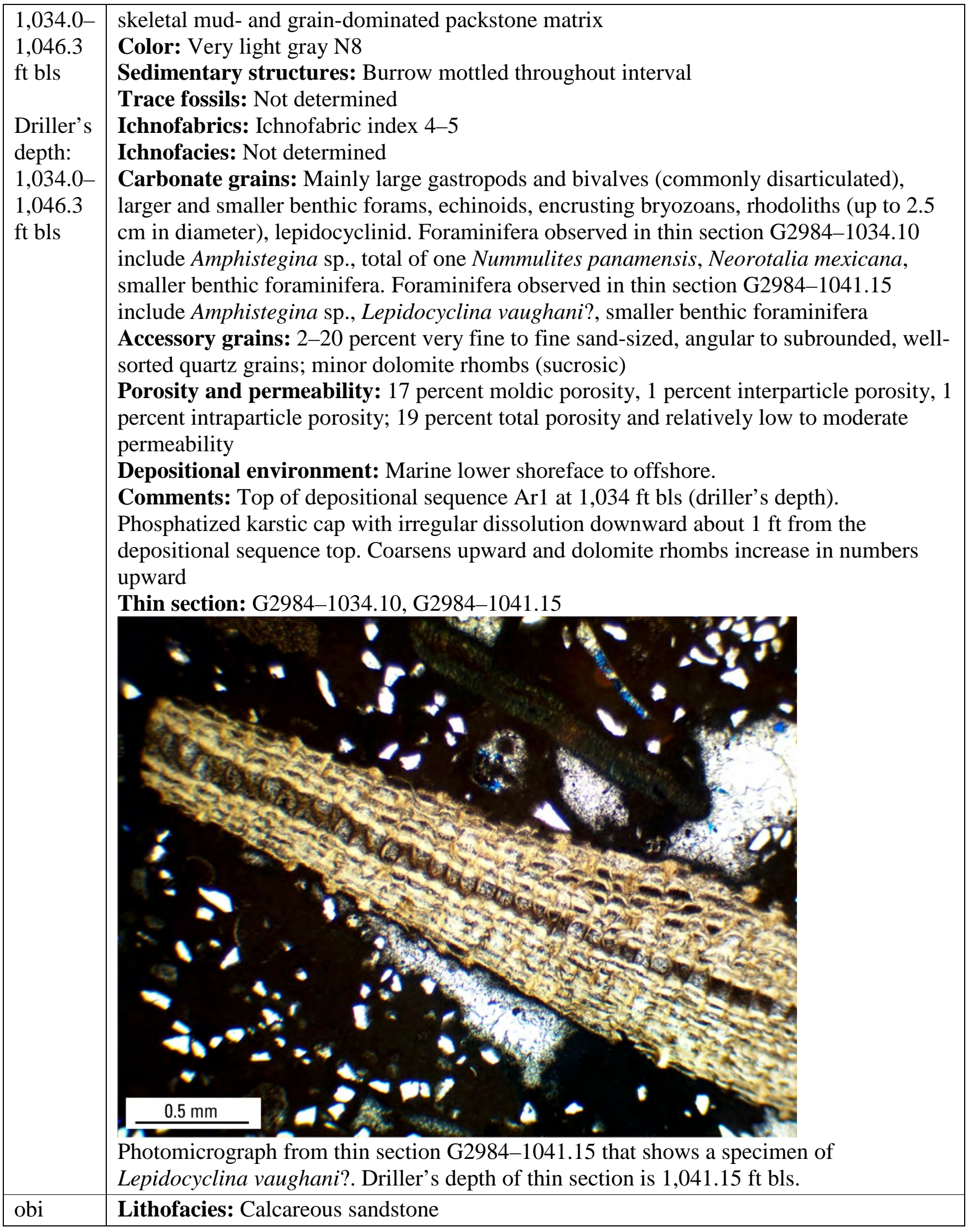




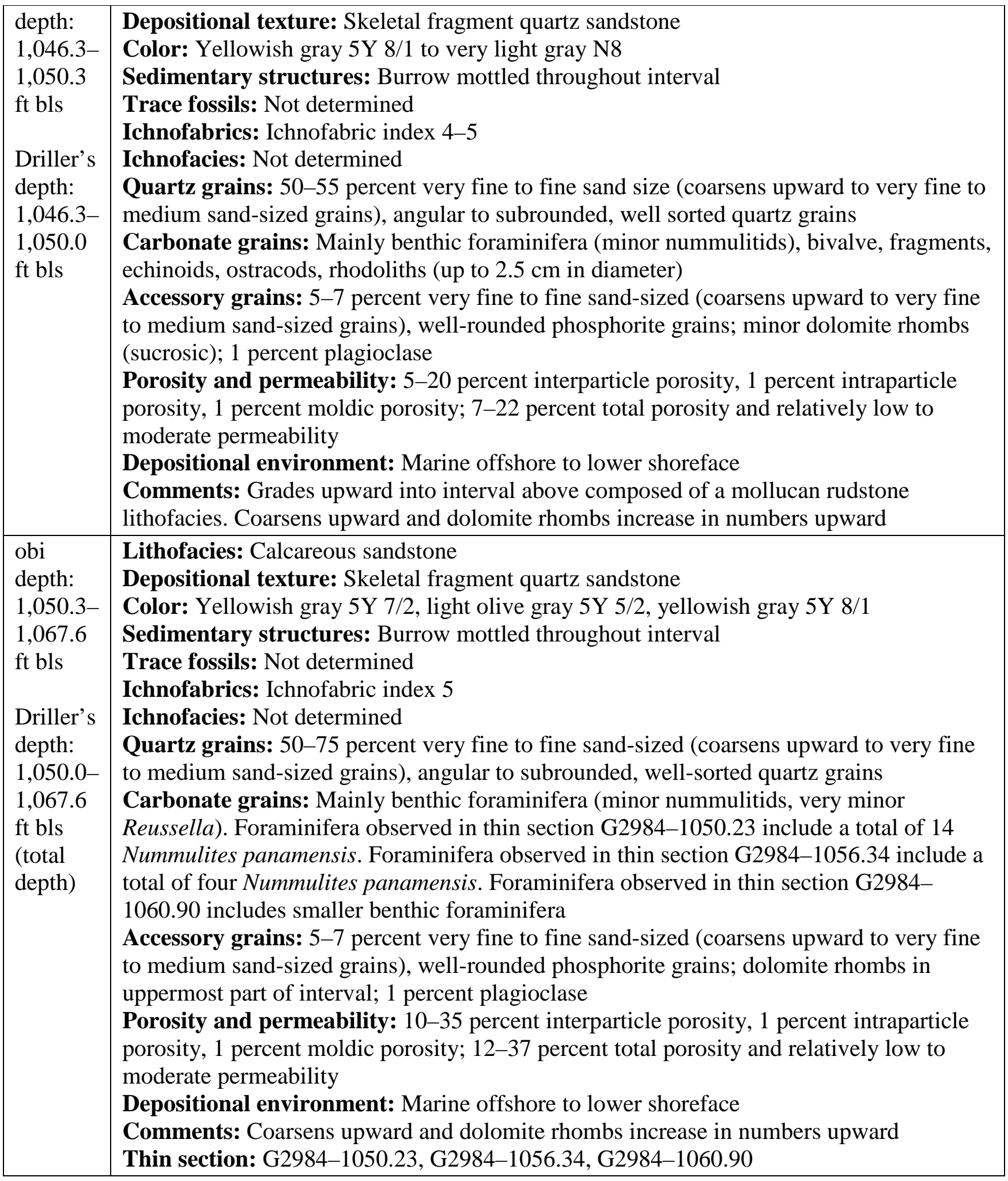




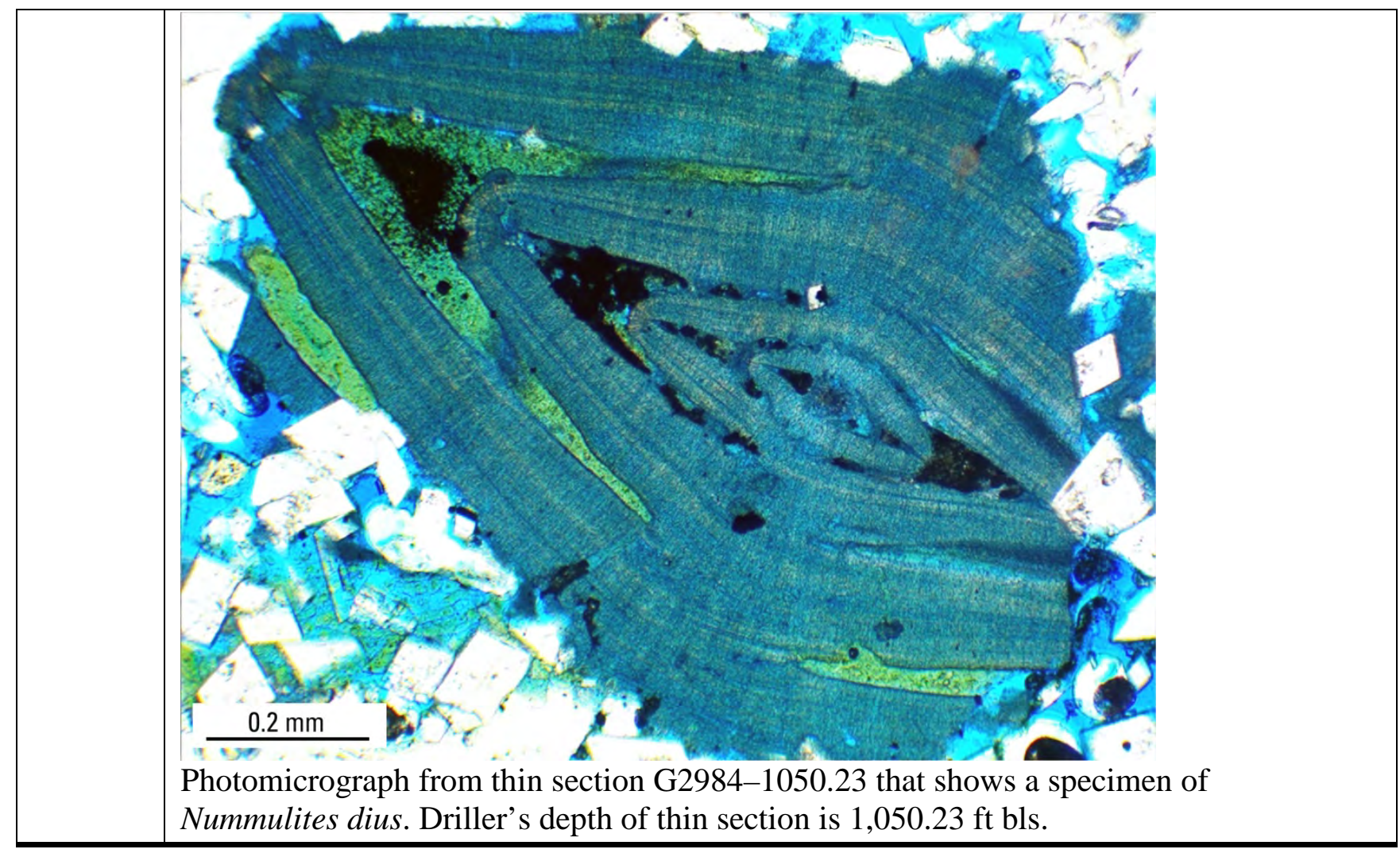




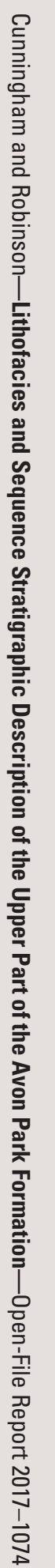

